\title{
$L$-FUNCTIONS OF $2 \times 2$ UNITARY GROUPS AND FACTORIZATION OF PERIODS OF HILBERT MODULAR FORMS
}

\author{
MICHAEL HARRIS
}

\section{INTRODUCTION}

The object of this paper is to prove, under mild local hypotheses, a conjecture of Shimura on the factorization of Petersson norms of Hilbert modular forms. A special case of the conjecture is the following. Let $E$ be a totally real field of degree $d$ over $\mathbb{Q}$, and let $\Sigma=\left\{\sigma_{1}, \ldots, \sigma_{d}\right\}$ be the set of real embeddings of $E$. Let $f$ be a Hilbert modular cusp form of weight $\left(k_{1}, \ldots, k_{d}\right)$, where $k_{1} \equiv k_{2} \equiv \cdots \equiv k_{d}(\bmod 2), k_{j} \geq 2$ for all $j$. Suppose $f$ is arithmetic in the sense that its Fourier expansion at every cusp has coefficients in $\overline{\mathbb{Q}}$, and that $f$ belongs to an irreducible automorphic representation $\pi$ of the adeles of $G L(2, E)$. Up to algebraic factors, the normalized Petersson norm $\langle f, f\rangle$ actually depends only on $\pi$, and not on the choice of arithmetic modular form in $\pi$. Suppose that, for $j=1, \ldots, d$, there exists a quaternion algebra $D(j)$ over $E$, which splits at $\sigma_{j}$ but is ramified at all other archimedean places, and an automorphic representation $\pi^{D(j)}$ of the adeles of $D(j)^{\times}$such that the local components $\pi_{v}^{D(j)}$ and $\pi_{v}$ are isomorphic for all $v$ at which $D(j)$ is unramified. It is equivalent to suppose that the system of Hecke eigenvalues associated to $f$ at almost all primes can be realized on the space of holomorphic modular forms of some weight on the Shimura curve $\mathscr{M}_{j}$ associated to $D(j)$. Let $f^{j}$ be an arithmetic modular form on $\mathscr{M}_{j}$ belonging to $\pi^{D(j)}$. In this case the curve $\mathscr{M}_{j}$ is compact, $f^{j}$ has no Fourier expansion, and the arithmetic modular forms are those identified by Shimura in [38] in terms of their values at special points on $\mathscr{M}_{j}$. The Petersson norm $\left\langle f^{j}, f^{j}\right\rangle$ is again an invariant of the representation $\pi^{D(j)}$, up to algebraic factors. Shimura conjectured, and we prove here, under a local hypothesis, that

$$
\text { The ratio }\langle f, f\rangle / \prod_{j=1}^{d}\left\langle f^{j}, f^{j}\right\rangle \text { is an algebraic number. }
$$

Shimura proved $(*)$ for $d=2$, and more generally showed in most cases how to write $\langle f, f\rangle$ as the product of two factors corresponding to a partition of $\Sigma$ into two pieces.

Received by the editors April 10, 1992 and, in revised form, August 24, 1992.

1991 Mathematics Subject Classification. Primary 11F41, 11F67, 11 F27.

Partially supported by National Science Foundation Grant No. DMS-8901101 and by the National Academy of Science exchange program with the USSR. 
More generally, if $D$ is any quaternion algebra over $E$, let $\Sigma(D)$ be the set of real places at which $D$ splits. Suppose there exists an automorphic representation $\pi^{D}$ of the adeles of $D^{\times}$such that the local components $\pi_{v}^{D}$ and $\pi_{v}$ are isomorphic for all $v$ at which $D$ is unramified. By the work of JacquetLanglands it is known that the existence of such $\pi^{D}$ is a purely local question: $\pi^{D}$ exists if and only if $\pi_{v}$ belongs to the discrete series of $G L\left(2, E_{v}\right)$ for every $v$ at which $D$ is ramified. Again, one can define the notion of arithmetic holomorphic modular forms in $\pi^{D}$. Our local hypothesis is that, for at least one finite prime $v_{0}, \pi_{v_{0}}$ belongs to the discrete series of $G L\left(2, E_{v}\right)$. Under this hypothesis, if $f^{D}$ is an arithmetic form, we prove Shimura's conjecture that

(**) The ratio $\left\langle f^{D}, f^{D}\right\rangle / \prod_{j \in \Sigma(D)}\left\langle f^{j}, f^{j}\right\rangle$ is an algebraic number.

The conjecture treated in the present article is one of a series of conjectures made by Shimura in $[42,43]$ regarding the periods of Hilbert modular forms. In the first place, Shimura conjectured the existence of a factorization, not only of the Petersson norms, but of the actual periods of Hilbert modular forms over cycles. We do not touch upon this question here; Shimura's work in [41] suggests that this question is related to the theory of modular forms of half-integral weight. In addition, Shimura proved in most cases the result, which evidently follows from $(* *)$, that $\left\langle f^{D}, f^{D}\right\rangle$ depends only on $\Sigma(D)$, up to algebraic factors. He then conjectured that, for each set $I$ of distinct real embeddings of $E$, there exists an invariant $q^{I}(\pi)$ which plays the role of the $\left\langle f^{D}, f^{D}\right\rangle$ even when there is no $D$ with $\Sigma(D)=I$ for which $\pi^{D}$ exists. In [9, I] we suggested a candidate, which we called $\nu^{I}(\pi)$, for this invariant. This invariant satisfies another of Shimura's conjectures; namely, the critical values of Rankin-Selberg convolutions of Hilbert modular forms can be expressed in terms of the $\nu^{I}(\pi)$. It remains to be shown that $\nu^{I}(\pi)$ is an algebraic multiple of $\left\langle f^{D}, f^{D}\right\rangle$ when $\pi^{D}$ exists with $\Sigma(D)=I$; this will be proved in $[9, \mathrm{II}]$.

A different point of view was stressed by Oda in $[29,30]$. To each $\pi^{D}$ one can associate a compatible system of $\lambda$-adic representations $\left\{M_{\lambda}\left(\pi^{D}\right)\right\}$ in the $l$-adic cohomology with twisted coefficients of the corresponding Shimura variety. Here $\{\lambda\}$ is the set of finite places of a certain number field $E(\pi)$, and the representations take $\operatorname{Gal}(\overline{\mathbb{Q}} / E(D)) \rightarrow G l\left(M_{\lambda}\left(\pi^{D}\right)\right)$, where $E(D)$ is a number field associated to $\Sigma(D)$. When $D=D(j), E(D)=\sigma_{j}(E) \subset \mathbb{R}$; when $D=M(2, E)$ is split, $E(D)=\mathbb{Q}$. It has been proved by Carayol that

$$
M_{\lambda}(\pi) \cong \bigotimes_{j=1}^{d} M_{\lambda}\left(\pi^{D(j)}\right) .
$$

Oda pointed out that the $M_{\lambda}\left(\pi^{D}\right)$ should be regarded as the $\lambda$-adic realizations of motives $M\left(\pi^{D}\right)$ attached to $\pi^{D}$, and that the Tate conjecture suggests that the Hodge structures associated to the de Rham and Betti realizations of $M(\pi)$ 
should also factor. The relation $(*)$ can be seen as a partial Hodge-de Rham analogue of Carayol's theorem.

Indeed, the theory of automorphic vector bundles [8, 27] allows us to define arithmetic modular forms over specific number fields. It should then be possible to prove a reciprocity law for the action of $\operatorname{Gal}(\overline{\mathbb{Q}} / \mathbb{Q})$ on the ratios in $(*)$ and $(* *)$. At least in principle, this is clear from the motivic point of view, and Panchishkin has formulated a conjecture along these lines in some generality [31]. At various times I have been convinced that the methods of this paper could easily be extended to prove such a reciprocity law, and I have even said as much in public. I continue to believe that the reciprocity law can be derived from the present methods, but it is certainly not as easy as I previously believed. This is primarily because my methods are based on the theory of the theta correspondence, whose definition only makes sense over the field $\mathbb{Q}^{\text {ab }}$ obtained by adjoining to $\mathbb{Q}$ all roots of unit. More precisely, if $D$ is a quaternion algebra over $E$ and $\mathscr{K}$ is a totally imaginary quadratic extension of $E$ which embeds in $D$, one can define a hermitian structure on $D$ as a $\mathscr{K}$-vector space, and the group $G U_{\mathscr{K}}(D)$ of unitary similitudes of $D$ is closely related to $D^{\times}$. If $D^{\prime}$ is another such algebra, one can study the theta correspondence from automorphic forms on $G U_{\mathscr{K}}\left(D^{\prime}\right)$ to automorphic forms on $G U_{\mathscr{K}}(D)$. When either $D^{\prime}$ or $D$ is split, the Schrödinger model and the $q$-expansion principle for Hilbert modular forms make it possible to finesse the problems arising from roots of unity; cf. [20, 39] for two such approaches (which do not, however, carry out the descent to finite extensions of $\mathbb{Q}$ ). However, the present proof of factorization is essentially an induction on the number of ramified places at infinity, and there are no simple formulas for the Schrödinger models in the general case.

The proof of Shimura's conjecture is rather convoluted, but the basic idea is not so hard to explain. An automorphic representation of $G U_{\mathscr{K}}(D)$ is actually given by a pair $\left(\pi^{D}, \omega\right)$, where $\pi^{D}$ is an automorphic representation of $D^{\times}$ and $\omega$ is a Hecke character of $\mathscr{K}_{\mathrm{A}}^{\times} / \mathscr{K}^{\times}$, with the property that the restriction of $\omega$ to $E_{\mathbf{A}}^{\times}$is the inverse of the central character of $\pi^{D}$. It turns out that the choice of $\omega$ plays a crucial role, both in the analytic theory of the theta correspondence and in its arithmetic properties. It is not difficult to prove that, if $\left(\pi^{D^{\prime}}, \omega\right)$ is an automorphic representation of $G U_{\mathscr{K}}\left(D^{\prime}\right)$, then its theta-lift $\Theta(\pi, \omega)$ to $G U_{\mathscr{K}}(D)$ either vanishes or equals $\left(\check{\pi}^{D}, \omega^{-1}\right)$, where $\pi^{D}$ and $\pi^{D^{\prime}}$ correspond via the Jacquet-Langlands correspondence and the $\check{\pi}^{D}$ is the contragredient of $\pi^{D}$. Following the pattern first observed by Waldspurger, the vanishing of $\Theta(\pi, \omega)$ is determined by local and global obstructions. As a first step, we prove in $\S 4$ in almost all cases that the local obstruction at $v$ is determined by the local root number of the $L$-function of $\pi_{\mathscr{K}} \otimes \omega$, where $\pi_{\mathscr{K}}$ is the base change of $\pi$ (or $\pi^{D^{\prime}}$ ) to an automorphic representation of $G L(2, \mathscr{K})$. This uses Shimizu's approach to the Jacquet-Langlands correspondence (also called the Jacquet-Langlands-Shimizu correspondence) and a theorem of Tunnell, recently extended by $\mathrm{H}$. Saito, on root numbers and characters of representations of $G L(2)$. 
The global obstruction is determined using a method invented by Rallis [34]. One wishes to compute the Petersson norm of a function in $\Theta\left(\pi^{D^{\prime}}, \omega\right)$. Using Kudla's method of seesaw pairs [21], an extension of the Weil-Siegel formula, along the lines of Kudla and Rallis [22, 24] and the doubling method of Piatetski-Shapiro and Rallis [6], one shows that this norm can be written as an Euler product. The unramified local factors have been determined in this case by J.-S. Li [26], and the essential part of the Euler product turns out to be the value at $s=\frac{1}{2}$ (the center of the critical strip) of $L\left(\pi_{\mathscr{K}} \otimes \omega, s\right)$. It now becomes necessary to show that one can choose $\omega$ so that $L\left(\pi_{\mathscr{H}} \otimes \omega, \frac{1}{2}\right) \neq 0$ and the local obstructions also vanish. This is the main result of [10]. Thus we can assume $\Theta(\pi, \omega) \neq 0$.

It should be mentioned that the extension of the Weil-Siegel formula mentioned above has not yet appeared in print. Although the generalization of the methods of Kudla and Rallis to unitary groups is not expected to present any difficulties, the cautious reader may wish to regard the main theorem as conditional.

As indicated, we now want to induct on $d-|\Sigma(D)|$, where $|*|$ indicates the cardinality. In order to do so, we have to assume there exist $D$, with $\Sigma(D)$ of arbitrary parity, such that $\pi^{D}$ exists. Thus we assume we have $D^{\prime}$ and $D$ with $\Sigma\left(D^{\prime}\right) \amalg\left\{\sigma_{j}\right\}=\Sigma(D)$, such that $\pi^{D^{\prime}}$ and $\pi^{D}$ exist, and satisfying some additional local conditions at finite places. Let $\omega$ be a Hecke character of $\mathscr{K}$ such that $\Theta\left(\pi^{D^{\prime}}, \omega\right) \neq 0$. Rallis' method compares the Petersson norm of an arithmetic form $f^{D^{\prime}} \in \pi^{D^{\prime}}$ with that of its theta lift $\theta_{\varphi}\left(f^{D^{\prime}}\right)$; here $\varphi$ is a variable Schwartz-Bruhat function defining the theta kernel. The main remaining step is to show that $\theta_{\varphi}\left(f^{D^{\prime}}\right)$ is arithmetic, up to factors which depend only on $\omega$. In doing so, we adapt an idea used by Shimura in [39]. To test for arithmeticity, we restrict $\theta_{\varphi}\left(f^{D^{\prime}}\right)$ to a subgroup of the form $U(1) \times U(1)$, integrate against a character of the latter subgroup, and divide the result by the CM period invariants introduced by Shimura in [38]. If the final result is an algebraic number, we are done.

Again we use seesaw pairs, to reduce this calculation to an integral of $f^{D^{\prime}}$ against a product of binary theta functions on $G U_{\mathscr{K}}\left(D^{\prime}\right)$. If $D^{\prime}$ were split we would be done, since the arithmetic of the binary theta functions on $G L(2)$ is well understood. But $D^{\prime}$ is not split, and we have to work out the theta lift \{characters of $U(1)\} \rightarrow$ automorphic forms on $\left.U\left(D^{\prime}\right)\right\}$ from scratch. This also turns out to be surprisingly subtle. The simplest method is to use Rallis' inner product formula again. Start with a Hecke character $\eta$ of $U(1)$. One again appeals to the Weil-Siegel formula, and one again finds that the Petersson norm of the lifted form is given by an $L$-value: in this case, the value of a Hecke $L$-series for $\mathscr{K}$ attached to a twisted base change to $\mathscr{K}^{\times}$of $\eta$.

The upshot of this argument is that we obtain a formula of the following type:

$$
\left\langle f^{D}, f^{D}\right\rangle \cdot p(\omega) / C \cdot\left\langle f^{D^{\prime}}, f^{D^{\prime}}\right\rangle \cdot L\left(\pi_{\mathscr{K}} \otimes \omega, \frac{1}{2}\right) \in \overline{\mathbb{Q}}^{\times}
$$

where $p(\omega)$ is a certain CM period attached to $\omega$ and $C$ is a constant which 
depends only on the archimedean data. After some additional fiddling using Shimura's earlier results (which we reprove in $\S 7$ ), we are able to verify that, if $D(j)$ is as in $(*)$, then

$$
C \cdot L\left(\pi_{\mathscr{K}} \otimes \omega, \frac{1}{2}\right) / p(\omega)\left\langle f^{D(j)}, f^{D(j)}\right\rangle \in \overline{\mathbb{Q}}^{\times} .
$$

This completes the proof of the induction step.

It should be mentioned that our notion of arithmetic forms is adapted to the canonical models of the quaternionic Shimura varieties attached to $D^{\times}$and not to those attached to $G U_{\mathscr{K}}(D)$. This is done for simplicity, but the reader may observe that the arithmeticity of the theta lifting is actually much better adapted to the canonical model of the Shimura variety attached to $G U_{\mathscr{K}}(D)$. It appears that the theta correspondence prefers some canonical models to others, and very much wants to come from something like an absolute Hodge cycle on a product of Shimura varieties. Similar phenomena have been observed in many other examples. The reasons for this deserve to be investigated, since there is no good reason a priori to expect the theta correspondence to have any reasonable arithmetic properties.

The first two sections develop the theory of CM periods and arithmetic automorphic forms on quaternionic Shimura varieties. Over $\overline{\mathbb{Q}}$, this theory is due to Shimura. The descent to the reflex field was carried out by the author in [8], and the descent to $\mathbb{Q}$ is due to Milne [27]. However, the material in the first two sections has not previously appeared in print. Although the results presented here are stronger than what we need for the proof of Shimura's conjecture over $\overline{\mathbb{Q}}$, the exposition is not greatly lengthened by the more precise treatment. The main theorem is stated as Theorem 2.6.1.

Section 3 introduces conventions for the theta correspondence. None of this material is new, but some subtleties arise due to our decision to work out the theta correspondence in the context of similitude groups. We follow the approach of $[12,13]$. An additional subtlety arises from the choice of a splitting of the metaplectic covering over the unitary dual reductive pair; this was brought to my attention by work of Gelbart-Rogawski [7]. Since the hermitian spaces $D$ and $D^{\prime}$ are of even dimension over $\mathscr{K}$, the Schrödinger model provides a natural splitting, but the problem resurfaces when we consider the lifting from $U(1)$ to $U\left(D^{\prime}\right)$. Section 4 works out the local theta lifting from $U_{\mathscr{K}}\left(D^{\prime}\right)$ to $U_{\mathscr{K}}(D)$.

The heart of the proof of the main theorem is contained in $\S 5$, which reduces the theorem to consequences of the Rallis inner product formula. This formula is proved, modulo the anticipated generalization of the Weil-Siegel formula of Kudla-Rallis, in $\S 6$, which also contains a description of the integral representation for the standard $L$-functions of unitary groups. Finally, $\S 7$ proves a formula for the special values of certain Rankin-Selberg convolutions in terms of CM periods and Petersson norms of quaternionic forms, including some cases not covered by previous results of Shimura; it also explains the relation between the main theorem and the transcendental part of the Birch-SwinnertonDyer conjecture for factors of Jacobians of Shimura curves. There is a brief appendix on the action of complex conjugation on CM periods. 


\section{Notation}

Notation for algebraic groups and adelic automorphic forms is standard and for the most part follows the conventions of $[13,9]$. We have especially adopted the conventions of [9], with one important difference in the global normalization of measures. Let $E$ be a totally real field, $\mathscr{K}$ a CM quadratic extension of $E$, $D$ a quaternion algebra over $E$, and $V$ a hermitian vector space over $\mathscr{K}$. If $G=R_{E / \mathbb{Q}} G L(2)_{E}, R_{E / \mathbb{Q}} D^{\times}, R_{E / \mathbb{Q}} G U(V)$ (unitary similitudes of $V$ ) or $R_{\mathscr{K} / \mathbb{Q}} \mathbb{G}_{m, \mathscr{K}}$, there is a natural embedding $R_{E / \mathbb{Q}} \mathbb{G}_{m, E} \rightarrow G$ as the maximal $E$-split torus in the center of $G$. We choose a global Haar measures $d g$ on $E_{\mathrm{A}}^{\times} G(\mathbb{Q}) \backslash G(\mathbf{A})$ such that

$$
\int_{E_{\mathbf{A}}^{\times} G(\mathbb{Q}) \backslash G(\mathbf{A})} d g=1
$$

In $\S 1.2$ measures are imposed on adele groups of more general tori, but these are of no importance in the sequel. Similarly, if $G=R_{E / \mathbb{Q}} S L(2)_{E}, R_{E / \mathbb{Q}} D^{1}$, $R_{E / \mathbb{Q}} U(V)$ (unitary group), where $D^{1} \subset D^{\times}$is the kernel of the reduced norm, then we require of our Haar measure on $G(\mathbb{Q}) \backslash G(\mathbf{A})$ that

$$
\int_{G(\mathbb{Q}) \backslash G(\mathbf{A})} d g=1
$$

This choice of measure is dictated by the desire to express the Weil-Siegel formula as simply as possible.

In $\S 6$ we are required to decompose $d g$ as a product $\prod d g_{v}$ of local measures, where $v$ runs through the places of $E$. Let $G(\mathbf{A})=\prod^{\prime} G_{v}$ (restricted direct product). For $v$ finite, we require that $d g_{v}$ take rational values on open compact subgroups, and, for almost all $v$, that the volume of a special maximal compact subgroup equal 1 . For $v$ a real place such that $G_{v} / E_{v}$ is anisotropic, we let $d g_{v}$ have total mass 1 on $G_{v} / E_{v}$. Finally, suppose $G_{v} \cong G L(2, \mathbb{R})=B(\mathbb{R}) \cdot S O(2)$, where $B$ is the upper triangular subgroup. Then $d g_{v}$ is the measure $(2 \pi)^{-1} \cdot d b \cdot d k$, where $d k$ is the Haar measure on $S O(2)$ with total volume 1 and $d b$ is the Haar measure on $B(\mathbb{R}) / \mathbb{R}^{\times}$which induces $y^{-2} d x d y$ on the quotient space $G L(2, \mathbb{R}) / \mathbb{R}^{\times} S O(2) \cong \mathbb{C}-\mathbb{R}$. The familiar formulas for the volumes of fundamental domains (for example, in [37, (2.29-30)]) make it clear that this is consistent with the previous normalization.

For (relative) ease of typesetting, the left action of $\operatorname{Gal}(\overline{\mathbb{Q}} / \mathbb{Q})$ on Shimura varieties and automorphic forms is written on the right. Thus if Sh is a Shimura variety and $\tau \in \operatorname{Gal}(\overline{\mathbb{Q}} / \mathbb{Q})$, the $\tau$-conjugate of $\mathrm{Sh}$, denoted $\mathrm{Sh}^{\tau}$ in the present manuscript, is what in [27] is denoted ${ }^{\tau} \mathrm{Sh}$. Thus $\mathrm{Sh}^{\tau \cdot \tau^{\prime}}=\left(\mathrm{Sh}^{\tau^{\prime}}\right)^{\tau}$.

\section{Periods of algebraic Hecke characters}

In this section we define analogues of the period invariants constructed in Part I, for cusp forms defining coherent cohomology classes on the Shimura varieties attached to quaternion algebras over $E$. In order to carry out this program, we have to explain what we mean by arithmetic holomorphic automorphic forms. The relevant constructions are contained implicitly in [8, II], refining Shimura's 
constructions in $[38,39]$ over $\overline{\mathbb{Q}}$. However, as we already discovered in [13], a more explicit method is desirable for applications to the theta correspondence. This approach which, with more foresight on the author's part, could have been included in [8], was sketched in the appendix to [13]; here we give a more complete treatment.

1.1. Let $(G, X)$ be a pair consisting of a reductive group $G / \mathbb{Q}$ and a $G(\mathbb{R})$ conjugacy class $X$ of homomorphism $R_{\mathbb{C} / \mathbb{R}} \mathbb{G}_{m, \mathbb{C}} \rightarrow G_{\mathbb{R}}$, satisfying the usual axioms defining a Shimura variety $\operatorname{Sh}(G, X)$, whose set of complex points is given by the familiar construction

$$
\operatorname{Sh}(G, X)(\mathbb{C})=\lim _{\longleftarrow} G(\mathbb{Q}) \backslash X \times G\left(\mathbf{A}^{f}\right) / K,
$$

where the limit runs over the set of open compact subgroups $K \subset G\left(\mathbf{A}^{f}\right)$ (cf. [8, 27]). Let $E(G, X)$ denote the reflex field of $(G, X)$, as in [27]; then $\operatorname{Sh}(G, X)$ has a canonical model over $E(G, X)$, and the natural action of $G\left(\mathbf{A}^{f}\right)$ on $\operatorname{Sh}(G, X)$ is rational over $E(G, X)$. We do not assume initially that the weight map $w: \mathbb{G}_{m, \mathbb{R}} \rightarrow G_{\mathbb{R}}$, defined as the composite

$$
\mathbb{G}_{m, \mathbb{R}} \rightarrow R_{\mathbb{C} / \mathbb{R}} \mathbb{G}_{m, \mathbb{C}} \stackrel{h}{\rightarrow} G_{\mathbb{R}},
$$

for any $h \in X$, is defined over $\mathbb{Q}$, nor that the maximal $\mathbb{Q}$-split subtorus of the center $Z_{G}$ of $G$ is of dimension $\leq 1$. A special pair is a pair $(G, X)=(H, h)$ where $G=H$ is a torus (and $X=h$ is thus a single point); a special pair is called a $C M$ pair if the corresponding weight map is defined over $\mathbb{Q}$. To any special pair $(H, h)$ we can associate a rational torus $\bar{H}$ and a surjective map $u: H \rightarrow \bar{H}$ such that $(\bar{H}, u \circ h)$ is a $\mathrm{CM}$ pair and $(H, h) \rightarrow(\bar{H}, u \circ h)$ is universal for maps to CM pairs. Let $H^{0}$ be the kernel of the map $H \rightarrow \bar{H}$.

As in $[27$, p. 330], for any $h \in X$ and any finite-dimensional $\mathbb{Q}$-rational representation $\rho: G \rightarrow G L(V), \rho \circ h: R_{\mathbb{C} / \mathbb{R}} \mathbb{G}_{m, \mathbb{C}} \rightarrow G L\left(V_{\mathbb{R}}\right)$ defines a Hodge structure on $V$; if $\rho$ is faithful then $h$ can be recovered from this Hodge structure. Our basic example of special pairs will be the following. We fix a totally real field $E$ of degree $d$ over $\mathbb{Q}$, and let $\Sigma$ denote the set of real embeddings of $E$, as in the introduction; the elements of $\Sigma$ are denoted $\sigma_{j}$, or simply $j, j \in\{1, \ldots, d\}$. Let $\mathscr{K}$ be a totally imaginary quadratic extension of $E$, and let $\Sigma_{\mathscr{K}}$ denote the set of complex embeddings of $\mathscr{K}$. Let $\Phi$ be a CM type of $\mathscr{K}$, i.e., a set of $d$ complex embeddings of $\mathscr{K}$ such that $\Sigma=\left.\Phi\right|_{E}$. If $\imath \in \operatorname{Gal}(\mathscr{K} / E)$ denotes complex conjugation, then the condition that $\Phi$ be a $\mathrm{CM}$ type is equivalent to saying that $\Phi \cap \imath \Phi=\varnothing, \Phi \cup \imath \Phi=\Sigma_{\mathscr{K}}$. Let $\Psi \subset \Sigma_{\mathscr{K}}$ be any subset such that $\Psi \cap \imath \Psi=\varnothing$, so that $\Psi$ can be completed to a CM type (generally in more than one way). Let $H_{\mathscr{K}}=R_{\mathscr{K} / \mathbb{Q}} \mathbb{G}_{m, \mathscr{K}} ;$ then $H_{\mathscr{K}}$ has a natural faithful $2 d$-dimensional representation over $\mathbb{Q}$ :

$$
\rho_{\mathscr{K}}: H_{\mathscr{K}} \rightarrow G L_{\mathbb{Q}}(\mathscr{K}) ; \quad \rho_{\mathscr{K}}(h)(a)=h a .
$$

Then $\left(\mathscr{K} \otimes_{\mathbb{Q}} \mathbb{C}, \rho_{\mathscr{K}, \mathbb{C}}\right) \cong \bigoplus_{\sigma \in \Sigma_{\mathscr{K}}}\left(\mathscr{K}_{\sigma}, \sigma\right)$, where $\mathscr{K}_{\sigma}$ denotes the completion of $\mathscr{K}$ at the valuation defined by $\sigma$. We let $h_{\Psi}: R_{\mathbb{C} / \mathbb{R}} \mathbb{G}_{m, \mathbb{C}} \rightarrow H_{\mathscr{K}, \mathbb{R}}$ be the homomorphism such that, in the induced Hodge structure on $\mathscr{K}$, the subspace 
$\mathscr{K}_{\sigma} \subset \mathscr{K} \otimes_{\mathbb{Q}} \mathbb{C}$ is of type $(-1,0)$ if $\sigma \in \Psi$, of type $(0,-1)$ if $\sigma \in \imath \Psi$, and of type $(0,0)$ otherwise.

One sees easily that $\left(H_{\mathscr{K}}, h_{\Psi}\right)$ is a special pair, and is a CM pair if and only if $\Psi$ is a CM type. Let $H_{E}=\operatorname{ker}\left(N_{E / \mathbb{Q}}\right): R_{E / \mathbb{Q}} \mathbb{G}_{m, E} \rightarrow \mathbb{G}_{m, \mathbb{Q}}$. We view $H_{E}$ as a subtorus of $H_{\mathscr{K}}$, and let $T_{\mathscr{K}}=H_{\mathscr{K}} / H_{E}$. Then $T_{\mathscr{K}}=\bar{H}_{\mathscr{K}}$ for any $\Psi$ such that $\Psi$ is not a CM type. We call a character $\chi \in X\left(H_{\mathscr{K}}\right)$ motivic if $\chi$ factors through $T_{\mathscr{K}}$. The group of characters $X\left(H_{\mathscr{K}}\right)$ over $\overline{\mathbb{Q}}$ is naturally isomorphic to the space of functions

$$
\Lambda \underset{\text { def }}{=}\left\{\lambda: \Sigma_{\mathscr{K}} \rightarrow \mathbb{Z}\right\} ; \quad \lambda \leftrightarrow \chi_{\lambda}, \quad \chi_{\lambda}(x)=\prod_{\tau} \tau(x)^{\lambda(\tau)}
$$

where the product is taken over the set of $\tau \in \Sigma_{\mathscr{K}}$. As explained in [1; 13, Appendix], the character $\chi_{\lambda}$ is motivic if and only if the motivic weight $w\left(\chi_{\lambda}\right):=-\lambda(\tau)-\lambda(l \tau)$ is independent of $\tau \in \Sigma_{\mathscr{K}}$. The automorphic weight of $\chi_{\lambda}$ is then defined to be the $d$-tuple $\underline{k}\left(\chi_{\lambda}\right)=\left(k\left(\chi_{\lambda}\right)_{1}, \ldots, k\left(\chi_{\lambda}\right)_{d}\right)$, where $k\left(\chi_{\lambda}\right)_{j}=\left|\lambda\left(l \tau_{j}\right)-\lambda\left(\tau_{j}\right)\right|+1$, if $\left.\tau_{j}\right|_{E}=\sigma_{j}$.

If $\tau \in \operatorname{Gal}(\overline{\mathbb{Q}} / \mathbb{Q})$, let $\Psi^{(\tau)}=\{\tau \circ \sigma, \sigma \in \Psi\}$. Then $h_{\Psi}(\tau)=\left(h_{\Psi}\right)^{(\tau)}$, in the notation of [4], and there are canonical isomorphisms [4, V]

$$
\operatorname{Sh}\left(H_{\mathscr{K}}, h_{\Psi}\right)^{\tau} \stackrel{\sim}{\rightarrow} \operatorname{Sh}\left(H_{\mathscr{K}}, h_{\Psi}(\tau)\right) \text {. }
$$

Let $(H, h)$ be any special pair. An algebraic Hecke character

$$
\omega: H(\mathbb{Q}) \backslash H(\mathbf{A}) \rightarrow \mathbb{C}^{\times}
$$

is a Hecke character whose restriction $\omega_{\infty}$ to $H(\mathbb{R})$ coincides with an algebraic character $\chi \in X(H)$. We call $\chi$ the infinity type of $\omega$, and we denote by $X(\chi)$ the set of algebraic Hecke characters of infinity type $\chi$. Let $H^{m}$ be the maximal $\mathbb{Q}$-anisotropic subtorus of $H$ which splits over $\mathbb{R} ; H^{m} \supset H^{0}$, defined above. We say $\chi$ is motivic if its restriction to $H^{m}$ is trivial; in this case the elements of $X(\chi)$ are also called motivic. If $\chi$ is motivic, $\omega \in X(\chi)$, then the motivic and automorphic weights of $\omega$, denoted $\underline{k}(\omega)$ and $w(\omega)$, respectively, are defined to be those of $\chi$.

Let $\Phi$ be a CM type of $\mathscr{K}$, as above, and, for each $\sigma_{j} \in \Sigma$, let $\tau_{j} \in \Phi$ be the element restricting to $\sigma_{j}$. The motivic character $\chi=\chi_{\lambda}$ of $H_{\mathscr{K}}$ is said to be compatible with $\Phi$ if $\lambda(\tau) \leq \lambda(\imath \tau)$ for all $\tau \in \Phi ; \chi$ is of type $\Phi$ if the inequalities are strict. More generally, if $\Psi \subset \Phi, \chi$ is compatible with $\Psi$ if $\lambda(\tau) \leq \lambda(l \tau)$ for all $\tau \in \Psi$, and strictly compatible with $\Psi$ if the inequalities are strict. Let $\omega \in X(\chi)$; then $\omega$ is compatible with (or strictly compatible with, or of type) $\Phi$ (or $\Psi$ ) if $\chi$ is. Evidently, if the motivic character $\chi$ is of type $\Phi$, then it can be reconstructed from the $(d+1)$-tuple $(\underline{k}(\omega), w(\omega))$, and we have, in the notation of [9, I, Corollary 1.2.9]

$$
E(\chi)=E(\underline{k}(\omega))=E(\underline{k}(\chi)) .
$$

1.2. As in [13, Appendix] any motivic $\chi \in X(H)$ defines an $H\left(\mathbf{A}^{f}\right)$-homogeneous line bundle $\left[V_{\chi}\right]$ on the Shimura variety $\operatorname{Sh}(H, h)$, with a canonical isomorphism

$$
\left[V_{\chi}\right](\mathbb{C}) \stackrel{\sim}{\rightarrow} \lim _{\longleftarrow} H(\mathbb{Q}) \backslash H(\mathbf{A}) \times V_{\chi}(\mathbb{C}) / H(\mathbb{R}) \times K
$$


Here the limit runs over the set of open compact subgroups $K \subset H\left(\mathbf{A}^{f}\right)$, and $V_{\chi}$ is the space of the representation $\chi$, with the obvious $H(\mathbb{Q})$ action. Then the $H\left(\mathbf{A}^{f}\right)$-equivariant line bundle $\left[V_{\chi}\right]$ has a canonical model over a certain extension $E(\chi)$ of the reflex field $E(H, h)[8,27]$. Let $M_{D R}(\chi)$ denote the set of $E(\chi)$-rational sections of $\left[V_{\chi}\right]$ over $\operatorname{Sh}(H, h)$. More generally, if $\tau \in \operatorname{Gal}(\overline{\mathbb{Q}} / \mathbb{Q})$, then $\left[V_{\chi}\right]^{\tau}$, as automorphic vector bundle over $\operatorname{Sh}\left(H, h^{(\tau)}\right)$, is canonically isomorphic to $\left[V_{\chi^{\tau}}\right]$, where $\chi \mapsto \chi^{\tau}$ is the natural left action on $X(H)$ [27].

We may canonically identify

$$
\begin{array}{r}
H^{0}\left(\operatorname{Sh}(H, h),\left[V_{\chi}\right]\right) \cong C_{\chi}:=\left\{f \in C^{\infty}\left(H(\mathbb{Q}) \backslash H(\mathbf{A}), V_{\chi}(\mathbb{C})\right) \mid\right. \\
f\left(t t_{\infty}\right)=\chi\left(t_{\infty}\right)^{-1} \cdot f(t), \\
\left.t \in H(\mathbf{A}), t_{\infty} \in H(\mathbb{R})\right\} .
\end{array}
$$

Denote this isomorphism Lift. In particular, any $\omega \in X(\chi)$ defines a linear form

$$
\begin{gathered}
L_{\omega}: H^{0}\left(\operatorname{Sh}(H, h),\left[V_{\chi}\right]\right) \rightarrow \mathbb{C}, \\
L_{\omega}(s)=\int_{H(\mathbb{Q}) \cdot H(\mathbb{R}) \backslash H(\mathbf{A})} \underline{\operatorname{Lift}}(s)(t) \cdot \omega(t) d t .
\end{gathered}
$$

Here and henceforward we normalize Haar measure on tori so that

$$
\begin{aligned}
\operatorname{vol}\left(H(\mathbb{Q}) \cdot H^{m}(\mathbf{A}) \backslash H(\mathbf{A})\right) & =\operatorname{vol}\left(H^{m}(\mathbb{Q}) \cdot H^{m}(\mathbb{R}) \backslash H(\mathbf{A})\right) \\
=\operatorname{vol}\left(H^{m}(\mathbb{R}) \backslash H(\mathbb{R})\right) & =1 .
\end{aligned}
$$

If $\omega \in X(\chi)$, let $E(\omega)$ denote the extension of $E(\chi)$ generated by the values of $\omega$ on $H\left(\mathbf{A}^{f}\right)$. The Galois group $\operatorname{Gal}(\overline{\mathbb{Q}} / E(\chi))$ acts on the set $X(\chi)$ by the formula

$$
\omega^{\sigma}(\gamma)=(\omega(\gamma))^{\sigma}, \quad \omega \in X(\chi), \gamma \in H\left(\mathbf{A}^{f}\right) .
$$

More generally, if $\sigma \in \operatorname{Gal}(\overline{\mathbb{Q}} / \mathbb{Q}), \chi$ is a motivic character of $X\left(H_{\mathscr{K}}\right)$, then $\chi^{\sigma}$ is also motivic, and if $\omega \in X(\chi)$, then $\omega^{\sigma}$, defined by the above formula, is the $H\left(\mathbf{A}^{f}\right)$-component of an element $\omega^{\sigma} \in X\left(\chi^{\sigma}\right)$.

As explained in [13, Appendix], the canonical local systems constructed in [8, $\S 4]$ allow us to associate to each $\omega \in X(\chi)$ a number $p(\omega) \in \mathbb{C}^{\times}$, well defined modulo $E(\omega)^{\times}$, such that the following lemma holds (cf. [13, Lemma A.6]):

1.3. Lemma. Let $s \in H^{0}\left(\operatorname{Sh}(H, h),\left[V_{\chi}\right]\right)$. Let $L$ be a finite extension of $E(\chi)$. Then $s \in M_{D R}(\chi) \otimes_{E(\chi)} L$ if and only if the following conditions are satisfied:

(i) For all $\omega \in X(\chi), p(\omega)^{-1} L_{\omega}(s) \in \overline{\mathbb{Q}}$.

(ii) For all $\sigma \in \operatorname{Gal}(\overline{\mathbb{Q}} / L),\left[p(\omega)^{-1} L_{\omega}(s)\right]^{\sigma}=p\left(\omega^{\sigma}\right)^{-1} L_{\omega^{\sigma}}(s)$.

More generally, let $\Sigma(\omega)$ denote the set of embeddings of $E(\omega)$ into $\overline{\mathbb{Q}}$ inducing the given embedding on $E(H, h)$. Then the vector $\left(p\left(\omega^{\sigma}\right)\right)_{\sigma \in \Sigma(\omega)}$ is well defined as an element of $\left(E(\omega) \otimes_{E(H, h)} \mathbb{C}\right)^{\times} / E(\omega)^{\times}$. 
1.3.1. Remark. If we allow the Shimura variety $\operatorname{Sh}(H, h)$ to vary among its $\operatorname{Gal}(\overline{\mathbb{Q}} / \mathbb{Q})$-conjugates, then $E(H, h)$ can be replaced by $\mathbb{Q}$, cf. [27]. The formulas for the action of $\operatorname{Gal}(\overline{\mathbb{Q}} / \mathbb{Q})$ on the set $\{\operatorname{Sh}(H, h)\}$ are given in (1.1.1).

If $L$ is a finite extension of the number field $L^{\prime}$ and $(a),(b) \in\left(L \otimes_{L^{\prime}}\right.$ $\mathbb{C})^{\times} \cup\{0\}$, we write $(a) \sim_{L}(b)$ if and only if $(a)=\alpha(b)$ for some $\alpha \in L^{\times}$. More generally, if $L \supset L^{\prime \prime} \supset L^{\prime}$ and $a=a\left(\gamma_{1}, \ldots, \gamma_{r}\right)$ and $b=b\left(\gamma_{1}, \ldots, \gamma_{r}\right)$ are functions of auxiliary data $\left\{\gamma_{1}, \ldots, \gamma_{r}\right\}$, such that $\operatorname{Gal}\left(\bar{L}^{\prime \prime} / L^{\prime \prime}\right)$ act on the set of $\left\{\gamma_{1}, \ldots, \gamma_{r}\right\}$, then we write $(a) \sim_{L ; L^{\prime \prime}}(b)$ if $(a) \sim_{L}(b)$ and if either (b) $=(0)$ or if

$$
\begin{aligned}
& {\left[a\left(\gamma_{1}, \ldots, \gamma_{r}\right) / b\left(\gamma_{1}, \ldots, \gamma_{r}\right)\right]^{\sigma}=\left[a\left(\gamma_{1}^{\sigma}, \ldots, \gamma_{r}^{\sigma}\right) / b\left(\gamma_{1}^{\sigma}, \ldots, \gamma_{r}^{\sigma}\right)\right]} \\
& \quad \text { for all } \sigma \in \operatorname{Gal}\left(\bar{L}^{\prime \prime} / L^{\prime \prime}\right) .
\end{aligned}
$$

The following abstract versions of Shimura's "monomial relations" [38, 39] follow immediately from the definitions:

1.4. Proposition. Let $(H, h)$ be a special pair, $\chi \in X(H)$ a motivic character, $\omega \in X(\chi)$. Define $\bar{H}, u: H \rightarrow \bar{H}$ as above.

(a) If $\chi=\bar{\chi} \circ u, \omega=\bar{\omega} \circ u_{\mathbf{A}}$, for some $\bar{\chi} \in X(\bar{H}), \bar{\omega} \in X(\bar{\chi})$, then $E(\omega)=E(\bar{\omega}) \cdot E(H, h)$ and

$$
\left(p\left(\omega^{\sigma}\right)\right)_{\sigma \in \Sigma(\omega)} \sim_{E(\omega) ; E(H, h)}\left(p\left((\bar{\omega})^{\sigma}\right)\right)_{\sigma \in \Sigma(\omega)}
$$

as elements of $\left(E(\omega) \otimes_{E(H, h)} \mathbb{C}\right)^{\times}$.

(b) Suppose $\left(H^{\prime \prime}, h^{\prime \prime}\right)=(H, h) \times\left(H^{\prime}, h^{\prime}\right)$, and let $\chi^{\prime \prime}=\chi \times \chi^{\prime}, \omega^{\prime \prime}=\omega \times \omega^{\prime}$ be the corresponding factorizations. Then $E\left(H^{\prime \prime}, h^{\prime \prime}\right)=E(H, h) \cdot E\left(H^{\prime}, h^{\prime}\right)$, $E\left(\omega^{\prime \prime}\right)=E(\omega) \cdot E\left(\omega^{\prime}\right)$, and

$$
\left(p\left(\left(\omega^{\prime \prime}\right)^{\sigma}\right)\right)_{\sigma \in \Sigma(\omega)} \sim_{E(\omega) ; E(H, h)}\left(p\left(\omega^{\sigma}\right) \cdot p\left(\left(\omega^{\prime}\right)^{\sigma}\right)\right)_{\sigma \in \Sigma(\omega)} .
$$

(c) With $(H, h)$ fixed, if $\omega=\omega^{\prime} \cdot \omega^{\prime \prime}$ is a product of two motivic Hecke characters, then $E(\omega) \subset E\left(\omega^{\prime}\right) \cdot E\left(\omega^{\prime \prime}\right)$, and

$$
\left(p\left(\omega^{\sigma}\right)\right)_{\sigma \in \Sigma(\omega)} \sim_{E(\omega) ; E(H, h)}\left(p\left(\left(\omega^{\prime}\right)^{\sigma}\right) \cdot p\left(\left(\omega^{\prime \prime}\right)^{\sigma}\right)\right)_{\sigma \in \Sigma(\omega)} .
$$

Proof. In general, a map of pairs $r:\left(H^{\prime}, h^{\prime}\right) \rightarrow(H, h)$ induces a pullback map $r^{*}: X(\chi) \rightarrow X(\chi \circ r)$ for any motivic $\chi \in X(H)$. Let $\omega \in X(\chi)$, and define $E(\omega), E(\omega \circ r), \Sigma(\omega), \Sigma(\omega \circ r)$ as above; note that $E(\omega \circ r) \supset E\left(H^{\prime}, h^{\prime}\right) \supset$ $E(H, h)$. Since formation of canonical models of automorphic vector bundles and of canonical local systems commutes with pullbacks (cf. [8, §4]), it is clear that

$$
\left(p\left(\omega^{\sigma}\right)\right)_{\sigma \in \Sigma(\omega \circ r)} \sim_{E(\omega \circ r) ; E(H, h)}\left(p(\omega \circ r)^{\sigma}\right)_{\sigma \in \Sigma(\omega \circ r)},
$$

and (a) is a special case of (1.4.1). The first two assertions of (b) are obvious from the definitions, whereas the last one is a consequence of the isomorphism $M_{D R}\left(\chi^{\prime \prime}\right) \cong M_{D R}(\chi) \otimes M_{D R}\left(\chi^{\prime}\right)$ (external tensor product) and the corresponding factorization of canonical local systems, cf. [8, Remark 4.5.4]. Finally, (c) follows from (b) with $\left(H^{\prime}, h^{\prime}\right)=(H, h)$, and from (1.4.1), applied to the diagonal map $(H, h) \rightarrow(H, h) \times(H, h)$. 
For our purposes, the most important corollary is the following application to the Shimura varieties $\left(H_{\mathscr{K}}, h_{\Psi}\right)$. Let $\omega$ be an algebraic Hecke character of $H_{\mathscr{H}}(\mathbb{Q}) \backslash H_{\mathscr{H}}(\mathbf{A}) \cong \mathscr{K}^{\times} \backslash \mathscr{H}_{\mathbf{A}}^{\times}$. The invariant $p(\omega)$ associated to $\omega$ and the pair $\left(H_{\mathscr{K}}, h_{\Psi}\right)$ obviously depends on $\Psi$. We write $p_{\mathscr{K}}(\omega, \Psi)$ instead of $p(\omega)$ to emphasize this dependence. Likewise, we write $E_{\mathscr{H}}(\omega, \Psi)$ instead of $E(\omega)$. Let $E_{\Psi}=E\left(H_{\mathscr{K}}, h_{\Psi}\right)$.

Suppose $\Psi$ is the disjoint union $\Psi^{\prime} \amalg \Psi^{\prime \prime}$ of two subsets of $\Sigma_{\mathscr{K}}$. Then it is easy to see that $E_{\Psi} \subset E_{\Psi^{\prime}} \cdot E_{\Psi^{\prime \prime}}$. The following proposition is a refinement of Shimura's [39, Theorem 1.1].

1.5. Corollary. Under the above hypotheses, let $\Sigma\left(\omega, \Psi^{\prime}, \Psi^{\prime \prime}\right)$ denote the set of embeddings of $E_{\mathscr{K}}\left(\omega, \Psi^{\prime}\right) \cdot E_{\mathscr{K}}\left(\omega, \Psi^{\prime \prime}\right)$ into $\overline{\mathbb{Q}}$, inducing the given embedding on $E_{\Psi} \cdot E_{\Psi^{\prime \prime}}$. Then

$$
\begin{aligned}
& \left(p_{\mathscr{K}}\left(\omega^{\sigma}, \Psi\right)\right)_{\sigma \in \Sigma\left(\omega, \Psi^{\prime}, \Psi^{\prime \prime}\right)} \\
& \quad \sim_{E_{\mathscr{H}}\left(\omega, \Psi^{\prime}\right) \cdot E_{\mathscr{H}}\left(\omega, \Psi^{\prime \prime}\right) ; E_{\Psi}}\left(p_{\mathscr{K}}\left(\omega^{\sigma}, \Psi^{\prime}\right) \cdot p_{\mathscr{K}^{\prime}}\left(\omega^{\sigma}, \Psi^{\prime \prime}\right)\right)_{\sigma \in \Sigma\left(\omega, \Psi^{\prime}, \Psi^{\prime \prime}\right)} .
\end{aligned}
$$

Proof. Our proof more or less follows that of Shimura. Consider the homomorphism $m: H_{\mathscr{K}} \times H_{\mathscr{K}} \rightarrow H_{\mathscr{K}}$ given by multiplication. Then

$$
m \circ\left(h_{\Psi^{\prime}} \times h_{\Psi^{\prime \prime}}\right)=h_{\Psi} .
$$

We consider $\omega \circ m$ as an algebraic Hecke character associated to the Shimura variety $\operatorname{Sh}\left(H_{\mathscr{H}} \times H_{\mathscr{K}}, h_{\Psi^{\prime}} \times h_{\Psi^{\prime \prime}}\right)$; then $E(\omega \circ m)=E_{\mathscr{H}}\left(\omega, \Psi^{\prime}\right) \cdot E_{\mathscr{K}}\left(\omega, \Psi^{\prime \prime}\right)$. Now formula (1.4.1) shows that

$$
\left(p\left(\omega^{\sigma}\right)\right)_{\sigma \in \Sigma\left(\omega, \Psi^{\prime}, \Psi^{\prime \prime}\right)} \sim_{E(\omega \circ m) ; E_{\Psi}}\left(p\left(\omega^{\sigma} \circ m\right)\right)_{\sigma \in \Sigma\left(\omega, \Psi^{\prime}, \Psi^{\prime \prime}\right)} .
$$

The corollary now follows from Proposition 1.4(b).

The following lemma is treated as an axiom by Shimura in [39].

1.6. Lemma. Under the above hypotheses, let $\omega^{l}(x)=\omega \circ l(x)$. For any $\Psi$ as above, $p_{\mathscr{K}}(\omega, l \Psi) \sim_{E(\omega) ; E_{\Psi}} p_{\mathscr{K}}\left(\omega^{l}, \Psi\right)$.

Proof. Note that $E\left(\omega^{t}\right)=E(\omega)$, since $\omega$ and $\omega^{t}$ take the same set of values. Now the map $l: H_{\mathscr{K}} \rightarrow H_{\mathscr{K}}$, viewed as an automorphism of algebraic groups over $\mathbb{Q}$, defines an isomorphism $\left(H_{\mathscr{K}}, h_{\Psi}\right) \stackrel{\sim}{\rightarrow}\left(H_{\mathscr{K}}, h_{l \Psi}\right)$, hence an isomorphism $l_{*}: \operatorname{Sh}\left(H_{\mathscr{K}}, h_{\Psi}\right) \stackrel{\sim}{\rightarrow} \operatorname{Sh}\left(H_{\mathscr{K}}, h_{l \Psi}\right)$. This in turn defines isomorphisms

$$
\imath^{*}: H^{0}\left(\operatorname{Sh}\left(H_{\mathscr{K}}, h_{l \Psi}\right),\left[V_{\chi}\right]\right) \stackrel{\sim}{\rightarrow} H^{0}\left(\operatorname{Sh}\left(H_{\mathscr{K}}, h_{\Psi}\right),\left[V_{\chi^{\prime}}\right]\right)
$$

for all $\chi$, where $\chi^{l}=\chi \circ l$, and

$$
L_{\omega^{l}}\left(l^{*}(s)\right)=L_{\omega}(s) \quad \text { for all } s \in H^{0}\left(\operatorname{Sh}\left(H_{\mathscr{K}}, h_{l \Psi}\right),\left[V_{\chi}\right]\right), \omega \in X(\chi) .
$$

The lemma is now clear.

1.7. Remark. (a) It is easy to see that, if $\omega, \omega^{\prime} \in X(\chi)$ for some $\chi \in X\left(H_{\mathscr{K}}\right)$, then $p_{\mathscr{K}}(\omega, \Psi) \sim_{\overline{\mathbb{Q}}} p_{\mathscr{K}}\left(\omega^{\prime}, \Psi\right) \sim p_{\mathscr{K}}(\chi, \Psi)$, where $p_{\mathscr{K}}(\chi, \Psi)$ is a well-defined element of $\mathbb{C}^{\times} / \overline{\mathbb{Q}}^{\times}$. We may identify $X\left(H_{\mathscr{K}}\right)$ with the free abelian group $I_{\mathscr{K}}$ on $\Sigma_{\mathscr{K}}$. Then if $|\Psi|=\sum_{\sigma \in \Psi} \sigma \in I_{\mathscr{K}}$, the $p_{\mathscr{K}}(\chi,|\Psi|)$ defined by Shimura 
in $[38, \S 1]$ coincides with our $\left\{p_{\mathscr{K}}(\chi, \Psi) / p_{\mathscr{K}}\left(\chi, l_{l} \Psi\right)\right]^{1 / 2}$. Note that we have assumed $\chi$ to be motivic; thus Shimura's invariants are apparently more general than ours. However, an inspection of Shimura's arguments shows that all of his periods can be obtained by taking products of half-integral powers of ours.

(b) As in Remark 1.3.1, the period relations in Proposition 1.4, Corollary 1.5, and Lemma 1.6 can all be made homogeneous with respect to $\operatorname{Gal}(\overline{\mathbb{Q}} / \mathbb{Q})$, if we take into account the conjugation by $\operatorname{Gal}(\overline{\mathbb{Q}} / \mathbb{Q})$ of the varieties $\operatorname{Sh}(H, h)$. We use this fact without comment in the applications.

1.8. The character $\chi_{\lambda}$, defined as in $\S 1.1$, is said to be critical $[1, \S 5.1]$ if $\lambda(\tau) \neq \lambda(l \tau)$ for all $\tau$. In particular, every critical character $\chi_{\lambda}$ is of type $\Phi_{\lambda}$, in the sense of $\S 1.2$, for a (unique) CM type $\Phi_{\lambda}$. The following theorem of Blasius is stated as Proposition A.10 in [13]; we refer to [loc.cit.] for an explanation of the terminology.

1.8.1. Proposition (Blasius, [1]). Suppose $\chi=\chi_{\lambda}$ is a critical character of $H_{\mathscr{K}}$, and let $\omega \in X(\chi)$. Let $L_{\mathscr{K}}(\omega, s)$ be the L-function of the Hecke character $\omega$, and let $s=m$ be a critical value of $L_{\mathscr{K}}(\omega, s)$, in the sense of Deligne. Let $D_{E}$ denote the absolute discriminant of $E$. Then

$$
L_{\mathscr{K}}(\omega, m) \sim_{E(\omega) ; E(\chi)} D_{E}^{1 / 2} \cdot(2 \pi i)^{d m} \cdot p\left(\omega, \Phi_{\lambda}\right) .
$$

In fact, $E(\chi)$ can be replaced by $\mathbb{Q}$ in the above statement; then of course $\omega$ runs through the elements of $\bigcup X\left(\chi^{\sigma}\right)$, where $\sigma$ runs through $\operatorname{Gal}(\overline{\mathbb{Q}} / \mathbb{Q})$, and $\Phi_{\lambda}$ varies among the CM types of $\mathscr{K}$. In place of $(2 \pi i)^{d m}$, one has to use $\varepsilon(\sigma)^{m} \cdot(2 \pi i)^{d m}$, where $\varepsilon(\sigma)$ is a $\{ \pm 1\}$-valued function on complex embeddings of $E(\omega) \cdot \mathscr{K}$, cf. [2, Corollary H.5].

Let $\varepsilon_{\mathscr{K}}$ denote the quadratic Dirichlet character of $E^{\times} \backslash E_{\mathrm{A}}^{\times}$, corresponding to the quadratic extension $\mathscr{K} / E$. The $L$-function $L_{\mathscr{K}}(\omega, s)$ can also be viewed as an automorphic $L$-function of $G=G L(2, E)$, by the following theorem of Jacquet-Langlands and Shalika-Tanaka, cited (in a slightly different form) as Theorem 4.6.1 of $[9, \mathrm{I}]$.

1.8.2. Proposition. (a) Let $v$ be a place of $E$. There is a map $\omega_{v} \mapsto \pi\left(\omega_{v}, \mathscr{K}_{v}\right)$ from characters of $\mathscr{K}_{v}^{\times}$to admissible irreducible representations of $G$, which preserves local L-factors, up to a shift:

$$
L\left(\pi\left(\omega_{v}, \mathscr{K}_{v}\right), s\right)=L_{\mathscr{K}_{v}}\left(\omega_{v}, s+\frac{1}{2}\right) .
$$

If $\omega=\bigotimes_{v} \omega_{v}$ is an automorphic representation (Hecke character) of $G L(1)_{\mathscr{K}}$, then $\pi(\omega, \mathscr{K})=\bigotimes_{v} \pi\left(\omega_{v}, \mathscr{K}_{v}\right)$ is an automorphic representation of $G$. The representations $\pi\left(\omega_{v}, \mathscr{K}_{v}\right)$ and $\pi\left(\omega_{v}^{\prime}, \mathscr{K}_{v}\right)$ are isomorphic if and only if $\omega_{v}^{\prime}=$ $\omega_{v}$ or $\omega_{v}^{\prime}=\omega_{v}^{l}$.

(b) If $\xi_{\omega}=\left.\omega\right|_{G L(1)_{E}}$, then the central character of $\pi(\omega, \mathscr{K})$ is $\xi_{\omega} \cdot \varepsilon_{\mathscr{K}} \cdot\|\cdot\|_{\mathbf{A}}$. The contragredient $\check{\pi}(\omega, \mathscr{K})$ is equal to $\pi\left(\omega^{-1}, \mathscr{K}\right) \otimes\|\cdot\|_{\mathbf{A}}^{-1}$.

(c) The representation $\pi(\omega, \mathscr{K})$ is cuspidal if and only if $\omega \neq \omega^{l}$.

An automorphic representation of $G$ is called motivic if it corresponds to a Hilbert modular form of weight $(\underline{k}, r)$, as in [9, I, 1.2]. Here $r \in \mathbb{Z}$ and 
$\underline{k}=\left(k_{1}, \ldots, k_{d}\right)$ is a $d$-tuple of positive integers, all congruent to $r(\bmod 2)$. To all subsets $I \subset \Sigma$, and to any motivic automorphic representation $\pi$ of $G$, we have associated in [9, I, Lemma 1.4.5] a certain numerical invariant $\nu^{I}(\pi)$; this is a nonzero complex number well defined up to multiplication by scalars in the field denoted $\mathbb{Q}(\pi, I)$ in $[9, I]$. We generalize these constructions to quaternion algebras in $\S 2$, below.

The representation $\pi(\omega, \mathscr{K})$ is motivic if $\omega$ is a motivic algebraic Hecke character; then $\pi(\omega, \mathscr{K})$ corresponds to a Hilbert modular form of weight $(\underline{k}(\omega), 1-w(\omega))$, in the notation of $\S 1.1[9, \mathrm{I}, 4.6]$. In this case, we write $\nu^{I}(\omega, \mathscr{K})=\nu^{I}(\pi(\omega, \mathscr{K}))$.

The following lemma may be taken as a normalization of the invariants $p_{\mathscr{K}}(\omega, \cdot)$ (which differs from Shimura's, cf. Remark 1.7(a)).

1.8.3. Lemma. For any subset $\Psi \subset \Sigma$,

$$
p_{\mathscr{K}}\left(\|\cdot\|_{\mathbf{A}}, \Psi\right) \sim_{\mathbb{Q}}(2 \pi i)^{-|\Psi|} .
$$

Proof. By Corollary 1.5, and Lemma 1.6, we may assume $|\Psi|=1$. The absolute value factors through the norm $N_{\mathscr{K} / \mathbb{Q}}: H_{\mathscr{K}} \rightarrow \mathbb{G}_{m, \mathbb{Q}}$, and we have a map of special pairs $N_{\mathscr{K} / \mathbb{Q}}:\left(H_{\mathscr{K}}, h_{\Psi}\right) \rightarrow\left(\mathbb{G}_{m, \mathbb{Q}}, N\right)$, in the notation of [5, Appendix]. By Proposition 1.4(a), this reduces the assertion to the corresponding assertion for this latter Shimura variety, and this is contained in [5, A.1.6].

Under certain hypotheses, the critical values of the Rankin-Selberg $L$-functions $\left.L\left(\pi(\omega, \mathscr{K}) \otimes \pi\left(\omega^{\prime}, \mathscr{K}\right), s\right)\right)$ have been computed in [9, I, 3$]$, in terms of these invariants. We consider two examples, which will be used in $\S 7$. Let $(\underline{k}, r)$ be a $(d+1)$-tuple with $k_{j} \equiv r(\bmod 2)$ for all $j$. Let $\left(a_{j}\right)$ be a $d$-tuple of nonnegative integers. Partition $\Sigma$ into disjoint subsets $I$ and $I^{\prime}$. Let $\omega$, $\omega^{\prime}$ be motivic Hecke characters of $\mathscr{K}$ such that $\xi_{\omega} \cdot \xi_{\omega^{\prime}}=1$, in the notation of 1.8.2(b), and:

$$
\begin{gathered}
\underline{k}(\omega)_{j}=k_{j}+1+2 a_{j}, \quad j \in I, \quad \underline{k}(\omega)_{j}=k_{j}-1, \quad j \in I^{\prime} ; \\
\underline{k}\left(\omega^{\prime}\right)_{j}=k_{j}+1+2 a_{j}, \quad j \in I^{\prime}, \quad \underline{k}\left(\omega^{\prime}\right)_{j}=k_{j}-1, \quad j \in I ; \\
w(\omega)=-w\left(\omega^{\prime}\right)-r .
\end{gathered}
$$

Furthermore, let $\Phi$ be a CM type, and assume $\omega$ and $\omega^{\prime}$ are compatible with $\Phi$. Then $\pi(\omega, \mathscr{K})$ and $\pi\left(\omega^{\prime}, \mathscr{K}\right)$ correspond to Hilbert modular forms of weights $(\underline{k}(\omega), 1-r)$ and $\left(\underline{k}\left(\omega^{\prime}\right), r+1\right)$, respectively, and Proposition 1.8.2(b) implies that $\xi_{\pi(\omega, \mathscr{K})} \cdot \xi_{\pi\left(\omega^{\prime}, \mathscr{K}\right)}=\|\cdot\|_{\mathbf{A}}^{2}$. Bearing in mind our normalizations of $L$-functions, we have

$$
L\left(\pi(\omega, \mathscr{K}) \otimes \pi\left(\omega^{\prime}, \mathscr{K}\right), s\right)=L_{\mathscr{K}}\left(\omega \cdot \omega^{\prime}, s\right) \cdot L_{\mathscr{K}}\left(\omega \cdot \omega^{\prime, l}, s\right),
$$

where $L_{\mathscr{K}}\left(\omega \cdot \omega^{\prime}, s\right)$ and $L_{\mathscr{K}}\left(\omega \cdot \omega^{\prime, l}, s\right)$ are $L$-functions of Hecke characters of $\mathscr{K}$. Write $\Phi=\Psi \amalg \Psi^{\prime}$, where $\left.\Psi\right|_{E}=I,\left.\Psi^{\prime}\right|_{E}=I^{\prime}$. It follows from the hypotheses that $\omega \cdot \omega^{\prime}$ and $\omega \cdot \omega^{\prime, l}$ are both critical, and that $\omega \cdot \omega^{\prime}$ is of type 
$\Phi$, whereas $\omega \cdot \omega^{\prime, l}$ if of type $\Phi^{\prime}:=\Psi \amalg \imath \Psi^{\prime}$. More precisely,

$$
\begin{aligned}
\underline{k}\left(\omega \cdot \omega^{\prime}\right)_{j} & =2\left(k_{j}+a_{j}\right)-1 \text { for all } j ; \\
\underline{k}\left(\omega \cdot \omega^{\prime, l}\right)_{j} & =3+2 a_{j} \text { for all } j .
\end{aligned}
$$

Thus, if $\chi$ (resp. $\chi^{\vee}$ ) is the infinity type of $\omega \cdot \omega^{\prime}$ (resp. $\omega \cdot \omega^{\prime, l}$ ), we have

$$
E(\chi) \cdot E\left(\chi^{\vee}\right)=E(\underline{k})
$$

by (1.1.2). Moreover, $\xi_{\omega} \cdot \xi_{\omega^{\prime}}=1$ implies that $\omega \cdot \omega^{\prime}$ and $\omega \cdot \omega^{\prime, l}$ are unitary characters; hence the $L$-functions $L_{\mathscr{K}}\left(\omega \cdot \omega^{\prime}, s\right)$ and $L_{\mathscr{K}}\left(\omega \cdot \omega^{\prime, l}, s\right)$ do not vanish for $\operatorname{Re}(s) \geq 1$. Finally, the $\Gamma$-factors of $L_{\mathscr{K}}\left(\omega \cdot \omega^{\prime}, s\right)$ and $L_{\mathscr{K}}\left(\omega \cdot \omega^{\prime, l}, s\right)$ are easily computed, and one verifies that $s=1$ is a critical value for each of these $L$-functions. By Blasius' Theorem 1.8.1 and (1.8.5), we thus have

$$
\begin{aligned}
0 & \neq L_{\mathscr{K}}\left(\omega \cdot \omega^{\prime}, 1\right) \cdot L_{\mathscr{K}}\left(\omega \cdot \omega^{\prime, l}, 1\right) \\
& \sim_{E(\omega) \cdot E\left(\omega^{\prime}\right) ; E_{\Phi}}(2 \pi i)^{2 d} \cdot p_{\mathscr{K}}\left(\omega \cdot \omega^{\prime}, \Phi\right) \cdot p_{\mathscr{K}}\left(\omega \cdot \omega^{\prime, l}, \Psi \amalg \iota \Psi^{\prime}\right) .
\end{aligned}
$$

Here we have used the obvious fact that $E\left(\omega \cdot \omega^{\prime}\right) \cdot E\left(\omega \cdot \omega^{\prime, l}\right) \subset E(\omega) \cdot E\left(\omega^{\prime}\right)$. On the other hand, by [9, I, Theorem 3.5.1], $s=1$ is a critical value for $L(\pi(\omega, \mathscr{K}) \otimes \pi(\tilde{\omega}, \mathscr{K}), s), \lambda=2$ in that theorem and we have the formula

$$
\begin{aligned}
& L\left(\pi(\omega, \mathscr{K}) \otimes \pi\left(\omega^{\prime}, \mathscr{K}\right), 1\right) \\
& \quad \sim_{E(\omega) \cdot E\left(\omega^{\prime}\right) \cdot E(\underline{k}(I)) ; E_{\Phi}}(2 \pi i)^{2 d} \cdot i^{d(1-r)} \nu^{I}(\omega, \mathscr{K}) \cdot \nu^{I^{\prime}}\left(\omega^{\prime}, \mathscr{K}\right) ;
\end{aligned}
$$

here we are using the obvious fact that $\mathbb{Q}(\pi(\omega, \mathscr{K}))$, defined as in $[9, \mathrm{I}]$, coincides with $E(\omega)$, and the power of $i$ is computed easily. The homogeneity of $(1.8 .7)$ with respect to $\mathrm{Gal}(\overline{\mathbb{Q}} / \mathbb{Q})$ corresponds to [9, I, (3.5.3)]. Combining (1.8.6) and (1.8.7), we have

1.8.8. Lemma. Under the above hypotheses

$$
\begin{aligned}
p_{\mathscr{K}}\left(\omega \cdot \omega^{\prime}, \Phi\right) \cdot p_{\mathscr{K}}\left(\omega \cdot \omega^{\prime, l}, \Psi \amalg \imath \Psi^{\prime}\right) \\
\quad \sim_{E(\omega) \cdot E\left(\omega^{\prime}\right) \cdot E(\underline{k}(I)) ; E_{\Phi}} i^{d(1-r)} \nu^{I}(\omega, \mathscr{K}) \cdot \nu^{I^{\prime}}\left(\omega^{\prime}, \mathscr{K}\right) .
\end{aligned}
$$

We now analyze the left-hand side of the above formula. Using Corollary 1.5 and Remark $1.7(\mathrm{~b})$, we have (with $*=E(\omega) \cdot E\left(\omega^{\prime}\right) \cdot E(\underline{k}(I))$ and with subscript $\mathscr{K}$ omitted):

$$
\begin{aligned}
& p\left(\omega \cdot \omega^{\prime}, \Phi\right) \cdot p\left(\omega \cdot \omega^{\prime, l}, \Psi \amalg \imath \Psi^{\prime}\right) \\
& \sim_{* ; E_{\Phi}} p\left(\omega \cdot \omega^{\prime}, \Psi\right) \cdot p\left(\omega \cdot \omega^{\prime}, \Psi^{\prime}\right) p\left(\omega \cdot \omega^{\prime, l}, \Psi\right) p\left(\omega \cdot \omega^{\prime, l}, l \Psi^{\prime}\right) \\
& \sim_{* ; E_{\Phi}} p(\omega, \Psi)^{2} \cdot p\left(\omega, \Psi^{\prime}\right) \cdot p\left(\omega, l \Psi^{\prime}\right) \cdot p\left(\omega^{\prime}, \Psi\right) \cdot p\left(\omega^{\prime, l}, \Psi\right) \\
& \quad \cdot p\left(\omega^{\prime}, \Psi^{\prime}\right) \cdot p\left(\omega^{\prime, l}, l \Psi^{\prime}\right),
\end{aligned}
$$

by Proposition $1.4(\mathrm{c})$. Applying Lemma 1.6 several times, we find that

$$
\begin{aligned}
& p\left(\omega \cdot \omega^{\prime}, \Phi\right) \cdot p\left(\omega \cdot \omega^{\prime, l}, \Psi \amalg l \Psi^{\prime}\right) \\
& \quad \sim_{* ; E_{\Phi}} p(\omega, \Psi)^{2} \cdot p\left(\omega, \Psi^{\prime}\right) \cdot p\left(\omega^{l}, \Psi^{\prime}\right) \cdot p(\omega, \Psi) \cdot p\left(\omega^{\prime, l}, \Psi\right) \cdot p\left(\omega^{\prime}, \Psi^{\prime}\right)^{2} \\
& \quad \sim_{* ; E_{\Phi}} p(\omega, \Psi)^{2} \cdot p\left(\omega^{\prime}, \Psi^{\prime}\right)^{2} \cdot p\left(\omega \cdot \omega^{l}, \Psi^{\prime}\right) \cdot p\left(\omega^{\prime} \cdot \omega^{\prime, l}, \Psi\right)
\end{aligned}
$$


by Proposition 1.4(c). Now

$$
\omega \cdot \omega^{l}=\omega \circ N_{\mathscr{K} / E}=\xi_{\omega} \circ N_{\mathscr{K} / E}, \quad \omega^{\prime} \cdot \omega^{\prime, l}=\left(\xi_{\omega} \circ N_{\mathscr{K} / E}\right)^{-1}
$$

For any Hecke character $\eta$ let $\tilde{\eta}=\eta / \eta^{l}$. Applying Proposition 1.4 (c) several more times, we obtain

$$
p\left(\omega \cdot \omega^{\prime}, \Phi\right) \cdot p\left(\omega \cdot \omega^{\prime, l}, \Psi \amalg l \Psi^{\prime}\right) \sim_{* ; E_{\Phi}} p(\tilde{\omega}, \Psi) \cdot p\left(\tilde{\omega}^{\prime}, \Psi^{\prime}\right) .
$$

Combining this with 1.8 .8 , we find

1.8.9. Lemma. Under the above hypotheses,

$$
p(\tilde{\omega}, \Psi) \cdot p\left(\tilde{\omega}^{\prime}, \Psi^{\prime}\right) \sim_{E(\omega) \cdot E\left(\omega^{\prime}\right) \cdot E(\underline{k}(I)) ; E_{\Phi}} i^{d(1-r)} \nu^{I}(\omega, \mathscr{K}) \cdot \nu^{I^{\prime}}\left(\omega^{\prime}, \mathscr{K}\right)
$$

1.9. We modify the above analysis; $I, I^{\prime}, \Psi, \Psi^{\prime}$ and $\omega$ are still as above, and take $\eta$, strictly compatible with $\Psi^{\prime}$ and compatible with $\Phi$, such that

$$
\xi_{\omega} \cdot \xi_{\pi(\eta, \mathscr{K})}=1 ; \quad \underline{k}(\eta)_{j}=k_{j} ; \quad w(\eta)=r
$$

In this case we write

$$
L(\pi(\eta, \mathscr{K}) \otimes \pi(\omega, \mathscr{K}), s)=L(\pi(\eta \omega, \mathscr{K}), s) L\left(\pi\left(\eta \omega^{l}, \mathscr{K}\right), s\right)
$$

Again, $\eta \omega$ and $\eta \omega^{\imath}$ are both critical, of types $\Phi=\Psi \amalg \Psi^{\prime}$ and $\Phi^{\prime}=\imath \Psi \amalg \Psi^{\prime}$, respectively; $s=1$ is a critical value for each of them, and

$$
\begin{gathered}
\underline{k}(\eta \omega)_{j}=2 k_{j}+2 a_{j}\left(\text { resp. } 2 k_{j}\right), \quad j \in I\left(\text { resp. } j \in I^{\prime}\right), \\
\underline{k}\left(\eta \omega^{l}\right)_{j}=2+2 a_{j}(\text { resp. } 2), \quad j \in I\left(\text { resp. } j \in I^{\prime}\right) .
\end{gathered}
$$

Moreover, $\xi_{\pi(\omega, \mathscr{K}), 0} \cdot \xi_{\pi(\eta, \mathscr{K}), 0}=\varepsilon_{\mathscr{K}}$, in the notation of [9, I, Theorem 3.5.1]. We make the hypothesis, which is no longer automatic, that

$$
L(\pi(\eta, \mathscr{K}) \otimes \pi(\omega, \mathscr{K}), 1)=L_{\mathscr{K}}(\eta \omega, 1) L_{\mathscr{K}}\left(\eta \omega^{l}, 1\right) \neq 0
$$

By Blasius' Theorem (1.8.1), and [9, I, Theorem 3.5.1], we thus have

$$
\begin{aligned}
& i^{r\left|I^{\prime}\right|+(1-r)|I|} \cdot G\left(\varepsilon_{\mathscr{K}}\right) \cdot \nu^{I^{\prime}}(\eta, \mathscr{K}) \cdot \nu^{I}(\omega, \mathscr{K}) \\
& \quad \sim_{E(\omega) \cdot E(\eta) \cdot E(\underline{k}(I)) ; E_{\Phi}}(2 \pi i)^{d} \cdot p(\eta \omega, \Phi) \cdot p\left(\eta \omega^{l}, \Phi^{\prime}\right) .
\end{aligned}
$$

Here $G\left(\varepsilon_{\mathscr{K}}\right)$ is the Gauss sum attached to $\varepsilon_{\mathscr{K}}[9, I, \S 3.5]$. The above analysis 
can now be repeated. We obtain

$$
\begin{aligned}
&(2 \pi i)^{d} \cdot p(\eta \omega, \Phi) \cdot p\left(\eta \omega^{l}, \Phi^{\prime}\right) \\
& \sim_{* ; E_{\Phi}} p\left(\eta^{2}, \Psi^{\prime}\right) \cdot p\left(\omega^{2}, \Psi\right) \cdot p\left(\eta \cdot \eta^{l}, \Psi\right) \cdot p\left(\omega \cdot \omega^{l}, \Psi^{\prime}\right) \\
& \sim_{* ; E_{\Phi}} p\left(\eta^{2}, \Psi^{\prime}\right) \cdot p\left(\omega^{2}, \Psi\right) \cdot p\left(\xi_{\eta} \circ N_{\mathscr{K} / E} \cdot\|\cdot\|, \Psi\right) \\
& \cdot p\left(\|\cdot\|^{-1}, \Psi\right) \cdot p\left(\xi_{\omega} \circ N_{\mathscr{K} / E}, \Psi^{\prime}\right) \\
& \sim_{* ; E_{\Phi}} p\left(\eta^{2}, \Psi^{\prime}\right) \cdot p\left(\omega^{2}, \Psi\right) \cdot p\left(\xi_{\eta} \circ N_{\mathscr{K} / E} \cdot\|\cdot\|, \Psi\right) \\
& \quad \cdot p\left(\|\cdot\|^{-1}, \Phi\right) \cdot p\left(\xi_{\omega} \circ N_{\mathscr{K} / E} \cdot\|\cdot\|, \Psi^{\prime}\right) \\
& \sim_{* ; E_{\Phi}} p\left(\eta^{2} \cdot\left(\xi_{\eta} \circ N_{\mathscr{K} / E}\right)^{-1}, \Psi^{\prime}\right) \cdot p\left(\omega^{2} \cdot\left(\xi_{\omega} \circ N_{\mathscr{K} / E}\right)^{-1}, \Psi\right) \cdot p\left(\|\cdot\|^{-1}, \Phi\right) \\
& \quad \quad\left(\operatorname{since~by~hypothesis~} \xi_{\omega} \cdot \xi_{\eta}=\|\cdot\|^{-1} \cdot \varepsilon_{\mathscr{K}}\right) \\
& \sim_{* ; E_{\Phi}} p\left(\tilde{\eta}, \Psi^{\prime}\right) \cdot p(\tilde{\omega}, \Psi) \cdot p\left(\|\cdot\|^{-1}, \Phi\right) \sim_{* ; E_{\Phi}}(2 \pi i)^{d} \cdot p\left(\tilde{\eta}, \Psi^{\prime}\right) \cdot p(\tilde{\omega}, \Psi)
\end{aligned}
$$

where the last identity follows from Lemma 1.8.3. Hence

1.9.5. Lemma. Under the above hypotheses,

$G\left(\varepsilon_{\mathscr{K}}\right)\left[i^{r\left|I^{\prime}\right|} \cdot \nu^{I^{\prime}}(\eta, \mathscr{K})\right] \cdot\left[i^{(1-r)|I|} \nu^{I}(\omega, \mathscr{K})\right] \sim_{E(\omega) \cdot E(\eta) \cdot E(\underline{k}(I)) ; E_{\Phi}} p\left(\tilde{\eta}, \Psi^{\prime}\right) \cdot p(\tilde{\omega}, \Psi)$.

\section{ARITHMETIC AUTOMORPHIC FORMS ON QUATERNION ALGEBRAS}

2.1. Quaternionic Shimura varieties and automorphic forms. Let $D$ be a quaternion algebra over $E$, and let $\Sigma(D) \subset \Sigma$ denote the set of real embeddings $\sigma$ of $E$ such that $D_{\sigma}:=D \otimes_{E, \sigma} \mathbb{R} \cong M(2, \mathbb{R})$. Let $\Sigma(D)^{\prime}=\Sigma-\Sigma(D)$. Let $N_{D}: D^{\times} \rightarrow E^{\times}$denote the reduced norm, $\operatorname{Tr}_{D}: D \rightarrow E$ the reduced trace. As in $[9, \mathrm{I}]$, we denote the elements of $\Sigma\left\{\sigma_{j}\right\}$ or $\{j\}$, where $j=1,2, \ldots, d$. Let $\mathbb{H}$ denote the algebra of Hamiltonian quaternions over $\mathbb{R}$. Write $D^{\times}$ for the reductive group $R_{E / \mathbb{Q}} D^{\times}$over $\mathbb{Q}$. There is an obvious action of $D^{\times}(\mathbb{R}) \cong G L(2, \mathbb{R})^{\Sigma(D)} \times \mathbb{H}^{\Sigma(D)^{\prime}}$ on $\left(\mathfrak{H}^{ \pm}\right)^{\Sigma(D)}$, where $\mathfrak{H}^{ \pm}=\mathbb{C}-\mathbb{R}$ is the union of the upper and lower half-planes, and it is known that the pair $\left(D^{\times},\left(\mathfrak{H}^{ \pm}\right)^{\Sigma(D)}\right)$ naturally defines a Shimura variety, which we denote $\mathscr{M}_{D}=\operatorname{Sh}\left(D^{\times},\left(\mathfrak{H}^{ \pm}\right)^{\Sigma(D)}\right)$ [36; 27, p. 336]. The reflex field $E\left(D^{\times},\left(\mathfrak{H}^{ \pm}\right)^{\Sigma(D)}\right)$, over which $\mathscr{M}_{D}$ has a canonical model, is the subfield of $\overline{\mathbb{Q}}$ fixed by the stabilizer of $\Sigma(D)$ in $\operatorname{Gal}(\overline{\mathbb{Q}} / \mathbb{Q})$. let $D^{(\tau)}$ be the quaternion algebra over $E$ ramified at the same set of finite places as $D$, such that

$$
\Sigma\left(D^{(\tau)}\right)=\{\tau \circ \sigma \mid \sigma \in \Sigma(D)\}
$$

A special case of the Langlands conjectures (cf. [4, 27]) states that any choice of special pair $(H, h) \subset\left(D^{\times},\left(\mathfrak{H}^{ \pm}\right)^{\Sigma(D)}\right)$ yields a canonical isomorphism

$$
\left(\mathscr{M}_{D}\right)^{\tau} \cong \mathscr{M}_{D^{(\tau)}}, \quad \tau \in \operatorname{Gal}(\overline{\mathbb{Q}} / \mathbb{Q}) \text {. }
$$


The choice of $(H, h)$ also canonically identifies $D^{\times}\left(\mathbf{A}^{f}\right) \cong D^{(\tau), \times}\left(\mathbf{A}^{f}\right)$, and the isomorphism (2.1.1) respects the actions of $D^{\times}\left(\mathbf{A}^{f}\right)$ with respect to this identification.

2.1.2. Lemma. Let $(H, h) \subset\left(D^{\times},\left(\mathfrak{H}^{ \pm}\right)^{\Sigma(D)}\right)$ be a special pair. Then $(H, h)$ is isomorphic to some special pair $\left(H_{\mathscr{K}}, h_{\Psi}\right)$, where $\mathscr{H}$ is a CM quadratic extension of $E$ over which $D$ splits and $\Psi \subset \Sigma_{\mathscr{K}}$ is a subset such that $\Psi \cap \imath \Psi=$ $\varnothing$ and $\left.\Psi\right|_{E}=\Sigma(D)$. Moreover, each such $\left(H_{\mathscr{K}}, h_{\Psi}\right)$ embeds in $\left(D^{\times},\left(\mathfrak{H}^{ \pm}\right)^{\Sigma(D)}\right)$.

Proof. This is well known and due to Shimura, cf. [40, II, §2].

Let $\left(H_{\mathscr{K}}, h_{\Psi}\right) \subset\left(D^{\times},\left(\mathfrak{H}^{ \pm}\right)^{\Sigma(D)}\right)$; after conjugating by an element of $D^{\times}(\mathbb{Q})$ we may and will assume that $h_{\Psi} \in\left(\mathfrak{H}^{+}\right)^{\Sigma(D)}$. If $\tilde{\sigma} \in \Psi$ restricts to $\sigma \in \Sigma(D)$, we use $\tilde{\sigma}$ to identify $\mathbb{C} \cong \mathscr{K}_{\sigma}:=\mathscr{K} \otimes_{E, \sigma} \mathbb{R}$. Then each $\sigma \in \Sigma(D)$ defines a map

$$
u_{\sigma}(\mathscr{K}, \Psi): \mathbb{C}^{\times} \cong \mathscr{K}_{\sigma}^{\times} \hookrightarrow D_{\sigma}^{\times} \cong G L(2, \mathbb{R}) .
$$

If $\sigma=\sigma_{j} \in \Sigma(D)$, we let $K_{j}^{+}=K_{j}^{+}(\mathscr{K}, \Psi)$ denote the image of the unit circle under the map (2.1.3), and let

$$
r_{j}(\theta)=r_{j}(\mathscr{K}, \Psi, \theta)=u_{\sigma}(\mathscr{K}, \Psi)\left(e^{i \theta}\right), \quad \theta \in \mathbb{R} / 2 \pi \mathbb{Z} .
$$

Let $\mathbb{H}^{1} \subset \mathbb{H}^{\times}$denote the kernel of the reduced norm. Let $k$ and $r$ be integers of the same parity and assume $k \geq 2$. We define $\pi^{\mathbb{H}}(k, r)$ denote an irreducible $(k-1)$-dimensional representation of $\mathbb{H}^{\times}$on which $z \in \mathbb{R}^{\times}=Z_{\mathbb{H}^{\times}}$ acts as $z^{r}$; since $\mathbb{H}^{1} \cong S U(2), \pi^{\mathbb{H}}(k, r)$ is unique up to isomorphism. If $\sigma_{j} \in \Sigma(D)^{\prime}$, let $K_{j}^{+}=K_{j}^{+}(\mathscr{K}, \Psi)$ be the subgroup of $D_{\sigma_{j}}^{\times}$corresponding to $\mathbb{H}^{1}$; denote by $\pi^{\mathbb{H}}(k)$ the restriction of $\pi^{\mathbb{H}}(k, r)$ to $K_{j}^{+}$(or to $\mathbb{H}^{1}$ ) and let $\pi^{\mathbb{H}}(k)^{\vee} \cong \pi^{\mathbb{H}}(k)$ denote the contragredient to $\pi^{\mathbb{H}}(k)$.

Fix the embedding $\left(H_{\mathscr{K}}, h_{\Psi}\right) \subset\left(D^{\times},\left(\mathfrak{H}^{ \pm}\right)^{\Sigma(D)}\right)$ as above. Let $(\underline{k}, r) \in \mathbb{Z}^{d+1}$ be a $(d+1)$-tuples as in $[9, \mathrm{I}]$; recall that $k_{i} \equiv r(\bmod 2), i=1, \ldots, d$. Let $K_{\infty}^{+}=K_{\infty}^{+}(\mathscr{K}, \Psi)=\prod_{j} K_{j}^{+}$. Let $L_{(\underline{k}, r)}^{D}$ denote the space of the representation $\prod_{j \in \Sigma(D)^{\prime}} \pi^{\mathbb{H}}\left(k_{j}\right)^{\vee}$, and define the representation

$$
z_{\infty} \cdot \prod_{j \in \Sigma(D)} r_{j}\left(\theta_{j}\right) \cdot \prod_{j^{\prime} \in \Sigma(D)^{\prime}} h_{j^{\prime}} \mapsto N_{E / \mathbb{Q}}\left(z_{\infty}\right)^{-r} \cdot \prod_{j \in \Sigma(D)} e^{-i k_{j} \theta_{j}} \bigotimes_{j^{\prime} \in \Sigma(D)^{\prime}} \pi^{\mathbb{H}}\left(k_{j}\right)^{\vee}\left(h_{j^{\prime}}\right) ;
$$

here $z_{\infty} \in Z_{D} \times(\mathbb{R}), r_{j}(\theta) \in K_{j}^{+}$for $j \in \Sigma(D)$, and $h_{j^{\prime}} \in K_{j^{\prime}}^{+}$for $j^{\prime} \in \Sigma(D)^{\prime}$.

As in $[9, \mathrm{I}, 1.2]$, we write $D_{\mathbb{R}}^{\times} \cong \prod_{j=1}^{d} D_{j}^{\times}, \operatorname{Lie}\left(D^{\times}\right)_{\mathbb{C}} \cong \bigoplus_{j=1}^{d} \operatorname{Lie}\left(D_{j}^{\times}\right)_{\mathbb{C}}$. Let $Z_{j}$ denote the center of $D_{j}^{\times}$. For $j \in \Sigma(D)$, let $\mathfrak{k}_{j}=\operatorname{Lie}\left(K_{j}^{\times}\right)_{\mathbb{C}}$, and write the Harish-Chandra decomposition

$$
\operatorname{Lie}\left(D_{j}^{\times}\right)_{\mathbb{C}} \cong \operatorname{Lie}\left(Z_{j}\right)_{\mathbb{C}} \oplus \mathfrak{k}_{j} \oplus \mathfrak{p}_{j}^{+} \oplus \mathfrak{p}_{j}^{-}
$$


As in [9, I, 1.2], let $\mathfrak{k}_{\infty}=\operatorname{Lie}\left(K_{\infty}^{+}\right), \mathfrak{p}^{+, D}=\bigoplus_{j \in \Sigma(D)} \mathfrak{p}_{j}^{+}, \mathfrak{p}^{-, D}=\bigoplus_{j \in \Sigma(D)} \mathfrak{p}_{j}^{-}$. Let $\mathscr{P}_{\mathscr{K}, \Psi}$ denote the connected subgroup of $D^{\times}$with complex Lie algebra $\operatorname{Lie}\left(Z_{D^{\times}}\right)_{\mathbb{C}} \oplus \mathfrak{k}_{\infty, \mathbb{C}} \oplus \mathfrak{p}^{-, D}$, and let $M_{D}$ be the homogeneous space $D^{\times} / \mathscr{P}_{\mathscr{K}, \Psi}$. Then $\mathscr{P}_{\mathscr{K}, \Psi}$ is a parabolic subgroup, $M_{D}$ is a complete variety with a natural model over $E\left(D^{\times},\left(\mathfrak{H}^{ \pm}\right)^{\Sigma(D)}\right)$, and there is a $D^{\times}(\mathbb{R})$-homogeneous holomorphic embedding

$$
\beta:\left(\mathfrak{H}^{ \pm}\right)^{\Sigma(D)} \rightarrow M_{D}
$$

sending $h_{\Psi}$ to the fixed point under $\mathscr{P}_{\mathscr{K}, \Psi}$.

The representation $\rho_{(\underline{k}, r)}^{D}$ of $Z_{D^{\times}}(\mathbb{R}) \cdot K_{\infty}^{+}$is algebraic, hence defines a representation-also denoted $\rho_{(\underline{k}, r)}^{D}$-of $Z_{D^{\times}}(\mathbb{C}) \cdot K_{\infty}^{+}(\mathbb{C})$, which in turn extends to a representation of $\mathscr{P}_{\mathscr{K}, \Psi}$ trivial on $R_{u} \mathscr{P}_{\mathscr{K}, \Psi}=\exp \left(\mathfrak{p}^{-, D}\right)$. Let $\mathscr{L}_{(\underline{k}, r)}^{D}$ be the $D^{\times}$-homogeneous vector bundle on $M_{D}$ associated to $\rho_{(\underline{k}, r)}^{D}$. Let $\underline{k}^{D}$ be the $d$-tuple such that $k_{j}^{D}=k_{j}$ if $j \in \Sigma(D), k_{j}^{D}=0$ if $j \in \Sigma(D)^{\prime}$. Then (cf. [9, I, 1.2.9]) $\mathscr{L}_{(\underline{k}, r)}^{D}$ is rational over $E\left(\underline{k}^{D}\right)$ as a homogeneous vector bundle. Applying the main theorem of [8], as in [9, I, $\S 1]$, we can define a $D^{\times}\left(\mathbf{A}^{f}\right)$-homogeneous, $E\left(\underline{k}^{D}\right)$-rational vector bundle $\mathscr{E}_{(\underline{k}, r)}^{D}=\left[\mathscr{L}_{(\underline{k}, r)}^{D}\right]$ over $\mathscr{M}_{D}$ :

$$
\mathscr{E}_{(\underline{k}, r)}^{D}(\mathbb{C}) \cong \lim D^{\times}(\mathbb{Q}) \backslash \beta^{*}\left(\mathscr{L}_{(\underline{k}, r)}^{D}\right) \times D^{\times}\left(\mathbf{A}^{f}\right) / K_{f}
$$

where, as usual, the limit is over the set of open compact subgroups $K_{f} \subset$ $D^{\times}\left(\mathbf{A}^{f}\right)$. Let $\underline{k}^{\tau}=\left(k_{1}^{\tau}, \ldots, k_{d}^{\tau}\right)$, where $k_{j}^{\tau}$ is the $\tau^{-1} \cdot \sigma_{j}$-index of $\underline{k}$ (there is a misprint in the definition of $\underline{k}^{\tau}$ in [9]). It follows from the results of Milne [27] that

2.1.6. Proposition. For any $\tau \in \operatorname{Gal}(\overline{\mathbb{Q}} / \mathbb{Q})$, there is a canonical isomorphism

$$
\left(\mathscr{E}_{(\underline{k}, r)}^{D}\right)^{\tau} \stackrel{\sim}{\rightarrow} \mathscr{E}_{(\underline{\underline{k}}}^{\left.D^{\tau}, r\right)}
$$

$\operatorname{over}\left(\mathscr{M}_{D}\right)^{\tau} \cong \mathscr{M}_{D^{(\tau)}}(c f .(2.1 .1))$.

Let $\mathscr{A}^{D}(k, r)$ denote the space of $\left(L_{(\underline{k}, r)}^{D}\right)^{*}$-valued automorphic forms $f$ on $D^{\times}(\mathbb{Q}) \backslash D^{\times}(\mathbf{A})$ such that

$$
\begin{gathered}
f\left(z_{\infty} g\right) N_{E / \mathbb{Q}}\left(z_{\infty}\right)^{r} \cdot f(g), \quad z_{\infty} \in Z_{D^{\times}}(\mathbb{R}), g \in D^{\times}(\mathbf{A}) ; \\
f\left(g \cdot k_{\infty}\right)=\prod_{j \in \Sigma(D)} e^{i k_{j} \theta_{j}} \bigotimes_{j^{\prime} \in \Sigma(D)^{\prime}} \pi^{\mathbb{H}}\left(k_{j}\right)\left(h_{j^{\prime}}\right) \cdot f(g) \\
\text { if } k_{\infty}=\prod_{j \in \Sigma(D)} r_{j}\left(\theta_{j}\right) \cdot \prod_{j^{\prime} \in \Sigma(D)^{\prime}} h_{j^{\prime}} \text { as in }(2.1 .4), g \in D^{\times}(\mathbf{A}) ; \\
\left.R\left(\mathfrak{p}^{-, D}\right) f \equiv 0 \quad \text { (notation as in }[9, \mathbf{I}, \S 1]\right) .
\end{gathered}
$$


When necessary, we write $\mathscr{A}^{D}(\underline{k}, r ; \mathscr{K}, \Psi)$ to emphasize the dependence on the latter data. There is a canonical isomorphism

$$
\underline{\text { Lift }}=\underline{\operatorname{Lift}}_{(\underline{k}, r)}^{D}: \Gamma\left(\mathscr{M}_{D}(\mathbb{C}), \mathscr{E}_{(\underline{k}, r)}^{D}\right) \stackrel{\sim}{\rightarrow} \mathscr{A}^{D}(\underline{k}, r) .
$$

A holomorphic automorphic form of type $(\underline{k}, r)$ for $D^{\times}$is a function on $D^{\times}(\mathbb{Q}) \backslash D^{\times}(\mathbf{A})$ of the form $\lambda \circ f$, where $f \in \mathscr{A}^{D}(\underline{k}, r)$ and $\lambda \in L_{(\underline{k}, r)}^{D}$; we denote the space of such forms $(L \circ \mathscr{A})^{D}(\underline{k}, r)$ (or $\left.(L \circ \mathscr{A})^{D}(\underline{k}, r ; \mathscr{K}, \Psi)\right)$, and refer to them as coefficients of $\mathscr{A}^{D}(\underline{k}, r)$.

2.2. Automorphic forms. Let $K_{\infty}=K_{\infty}(\mathscr{K}, \Psi)$ denote the (unique) maximal compact subgroup of the normalizer in $D_{\mathbb{R}}^{\times}$of $K_{\infty}^{+}$, and define $K_{j} \subset D_{j}^{\times}$ analogously. Let $\left(\pi^{D}, H_{\pi^{D}}\right)$ be an irreducible cuspidal automorphic representation of $D^{\times}(\mathbf{A})$ which is generated by a holomorphic automorphic form of type $(\underline{k}, r)$ for $D^{\times}$. Then $\pi^{D}$ factors as a (restricted) tensor product $\bigotimes_{v} \pi_{v}^{D}$ of representations of $D_{v}^{\times}$; here $v$ runs over places of $E$, and $\pi_{v}^{D}$ is a $\left(\operatorname{Lie}\left(D_{v}^{\times}\right), K_{v}\right)$-module if $v$ is archimedean. If $v$ corresponds to the real embedding $\sigma_{j} \in \Sigma(D)$ (resp. $\sigma_{j^{\prime}} \in \Sigma(D)^{\prime}$ ) then $\pi_{v} \cong \pi\left(k_{j}, r\right)$, in the notation of $\S 1.1$ (resp. $\pi_{v} \cong \pi^{\mathbb{H}}\left(k_{j^{\prime}}, r\right)$ ).

The Jacquet-Langlands correspondence $[17, \S 16]$ associates to $\left(\pi^{D}, H_{\pi^{D}}\right)$ an irreducible cuspidal automorphic representation $\left(\pi, H_{\pi}\right)$ of $G(\mathbf{A})=G L\left(2, E_{\mathbf{A}}\right)$ such that, if $\pi \cong \bigotimes_{v} \pi_{v}$, then $\pi_{v}^{D} \cong \pi_{v}$ for all $v$ such that $D_{v}^{\times} \cong G_{v}$. We define $\mathbb{Q}(\pi)$ to be the field of definition of the representation $\pi_{f}$ as in $[9, I$, 1.3], and define $\mathbb{Q}\left(\pi^{D}\right)$ analogously. More generally, if $S$ is any finite set of nonarchimedean primes, let

$$
\pi_{f, S}=\bigotimes_{\substack{v \notin S \\ v \text { finite }}} \pi_{v}, \quad \pi_{f, S}^{D}=\bigotimes_{\substack{v \notin S \\ v \text { finite }}} \pi_{v}^{D},
$$

and let $\mathbb{Q}(\pi, S) \subset \mathbb{C}$ be the field fixed by $\sigma \in \operatorname{Aut}(\mathbb{C})$ such that $\pi_{f, S}^{\sigma} \cong \pi_{f, S}$; define $\mathbb{Q}\left(\pi^{D}, S\right)$ analogously. Then it follows from strong multiplicity one for $G$ (and the Jacquet-Langlands correspondence) that

$$
\mathbb{Q}(\pi, S)=\mathbb{Q}(\pi), \quad \mathbb{Q}\left(\pi^{D}, S\right)=\mathbb{Q}\left(\pi^{D}\right) \text { for any finite } S
$$

(compare [47, Corollary I.8.3]). In particular, letting $S$ be the set of finite primes for which $D$ is ramified, we find

$$
\mathbb{Q}\left(\pi^{D}\right)=\mathbb{Q}(\pi)
$$

(cf. [47, Lemma II.1.1]). It is known (cf. [37]) that $\mathbb{Q}(\pi)$ is either totally real or a CM field.

The isomorphism (2.1.10) defines an embedding

$$
\pi_{f}^{D} \cong\left(L_{(\underline{k}, r)}^{D} \otimes \pi^{D}\right)^{K_{\infty}^{+}} \hookrightarrow \Gamma\left(\mathscr{M}_{D}(\mathbb{C}), \mathscr{E}_{(\underline{k}, r)}^{D}\right) .
$$

Denote by $\Gamma\left(\mathscr{M}_{D}(\mathbb{C}), \mathscr{E}_{(\underline{k}, r)}^{D}\right)^{\pi}$ the image of this map. Let $\mathbb{Q}(\pi, D)=\mathbb{Q}(\pi) \cdot$ $E\left(\underline{k}^{D}\right)$. 
2.2.4. Proposition. (a) There is a natural $\mathbb{Q}(\pi, D)$-rational structure on the subspace $\Gamma\left(\mathscr{M}_{D}(\mathbb{C}), \mathscr{E}_{(\underline{k}, r)}^{D}\right){ }^{\pi} \cong \pi_{f}^{D}$ of $\Gamma\left(\mathscr{M}_{0}(\mathbb{C}), \mathscr{E}_{(\underline{k}, r)}^{D}\right)$, compatible with the $E\left(\underline{k}^{D}\right)$ rational structure on $\Gamma\left(\mathscr{M}_{D}(\mathbb{C}), \mathscr{E}_{(\underline{k}, r)}^{D}\right)$.

(b) For $\tau \in \operatorname{Gal}(\overline{\mathbb{Q}} / \mathbb{Q})$,

$$
\tau\left(\Gamma\left(\mathscr{M}_{D}(\mathbb{C}), \mathscr{E}_{(\underline{k}, r)}^{D}\right)^{\pi}\right)=\Gamma\left(\mathscr{M}_{D^{(\tau)}}(\mathbb{C}), \mathscr{E}_{(\underline{\underline{k}}, r)}^{D^{(\tau)}}\right)^{\pi^{(\tau)}} .
$$

Proof. Let $S_{f}(D)$ be the set of nonarchimedean places at which $D$ ramifies. For $v \notin S_{f}(D), v$ finite, fix an isomorphism $D_{v}^{\times} \cong G L\left(2, E_{v}\right)$ and, for $n \geq 0$, let

$$
K_{1}\left(m_{v}^{n}\right)=\left\{g \in D_{v}^{\times}=G L\left(2, E_{v}\right) \mid g \equiv\left[\begin{array}{cc}
* & * \\
0 & 1
\end{array}\right]\left(\bmod m_{v}^{n}\right)\right\},
$$

where $m_{v}$ is the maximal ideal in the ring of integers $\mathscr{O}_{v}$ of $E_{v}$. Let $c(v)$ be the smallest integer such that $\pi_{v}^{D} \cong \pi_{v}$ has fixed vectors under $K_{1}\left(m_{v}^{n}\right)$; then $c(v)=0$ for almost all $v$ and, by the theory of new forms, the subspace $\pi_{v}^{D}$ (new) of $K_{1}\left(m_{v}^{c(v)}\right)$-fixed vectors in $\pi_{v}^{D}$ is one-dimensional. Let $K=$ $K_{f}\left(\pi^{D}\right)=\prod_{v \notin S_{f}(D)} K_{1}\left(m_{v}^{c(v)}\right)$; then the $K_{f}\left(\pi^{D}\right)$-fixed subspace

$$
\left(\pi_{f}^{D}\right)^{K} \cong \bigotimes_{v \in S_{f}(D)} \pi_{v}^{D}
$$

Now let $W=\Gamma\left(\mathscr{M}_{D}, \mathscr{E}_{(\underline{k}, r)}^{D}\right)(\mathbb{Q}(\pi, D)), W_{0}=W^{K}$. Then $W_{0}$ is a $\mathbb{Q}(\pi, D)$ rational subspace of $W$. Let $T$ be the finite set of nonarchimedean primes containing $S_{f}(D)$ and all $v$ such that $c(v)>0$. For $v \notin T$, let $\mathscr{H}_{v}$ denote the local Hecke algebra at $v$, with coefficients in $\mathbb{Q}(\pi, D)$, relative to $G L\left(2, \mathscr{O}_{v}\right)$; let $\mathscr{H}=\bigotimes_{v \notin T} \mathscr{H}_{v}$. Then $\mathscr{H}$ acts on $\left(\pi_{f}^{D}\right)^{K}$ through a character $\alpha_{\pi}: \mathscr{H} \rightarrow$ $\mathbb{Q}(\pi) \cdot E\left(\underline{k}^{D}\right)$. Let $W_{0}(\pi) \subset W_{0}$ denote the $\alpha_{\pi}$-eigenspace for the natural action of $\mathscr{H}$ on $W_{0}$. Then $W_{0}(\pi)$ is rational over $\mathbb{Q}(\pi, D)$ since the $D^{\times}\left(\mathbf{A}^{f}\right)$-action on $\Gamma\left(\mathscr{M}_{D}, \mathscr{E}_{(\underline{k}, r)}^{D}\right)$ is $E\left(\underline{k}^{D}\right)$-rational. It follows from strong multiplicity one and (2.2.4.2) that $W_{0}(\pi)$ is a $\prod_{v \in S_{f}(D)} D_{v}^{\times}$-invariant $\mathbb{Q}(\pi, D)$-form of $\bigotimes_{v \in S_{f}(D)} \pi_{v}^{D}$. Now elementary arguments show that the $D^{\times}\left(\mathbf{A}^{f}\right)$-translates of $W_{0}(\pi)$ generate a $\mathbb{Q}(\pi, D)$-form of $\Gamma\left(\mathscr{M}_{D}, \mathscr{E}_{(\underline{k}, r)}^{D}\right)^{\pi}$. This completes the proof of (a), and (b) follows easily from Proposition 2.1.6 and strong multiplicity one.

As explained in [13, Appendix], the $L$-rational elements of $\Gamma\left(\mathscr{M}_{D}, \mathscr{E}_{(\underline{k}, r)}^{D}\right)$, for any $L \supset \mathbb{Q}(\pi, D)$, can be recognized in terms of their restrictions to the Shimura subvarieties $\operatorname{Sh}\left(H_{\mathscr{H}}, h_{\Psi}\right)$ and their $D^{\times}\left(\mathbf{A}^{f}\right)$-translates, for different $(\mathscr{K}, \Psi)$, satisfying the conditions of Lemma 2.1.2. We fix such a pair $(\mathscr{K}, \Psi)$ and the corresponding space $(L \circ \mathscr{A})^{D}(\underline{k}, r)$. Now the space $L_{(\underline{k}, r)}^{D}$ admits a finite-dimensional algebraic representation $\rho=\left.\rho_{(\underline{k}, r)}^{D}\right|_{H_{\mathscr{H}}}$ of $H_{\mathscr{K}}$. Say $\rho \cong$ $\bigoplus_{i=1}^{m} \chi_{i}$ over some finite extension $E^{\prime}$ of $E\left(\underline{k}^{D}\right)$, where each $\chi_{i} \in X\left(H_{\mathscr{H}}\right)$. Let

$$
X(\rho)=X\left(\chi_{1}\right) \times \cdots \times X\left(\chi_{m}\right) .
$$


Any $\omega=\left(\omega_{1}, \ldots, \omega_{m}\right) \in X(\rho), \omega_{i} \in X\left(\chi_{i}\right)$, defines a linear form

$$
L_{\omega}=\left(L_{\omega_{1}}, \ldots, L_{\omega_{m}}\right): H^{0}\left(\operatorname{Sh}\left(H_{\mathscr{K}}, h_{\psi}\right), i^{*}\left[\mathscr{E}_{(\underline{k}, r)}\right]\right) \rightarrow \mathbb{C}^{m}
$$

where $i: \operatorname{Sh}\left(H_{\mathscr{K}}, h_{\Psi}\right) \rightarrow \mathscr{M}_{D}$ is the natural embedding. Let

$$
p(\omega)^{-1} L_{\omega}=p_{\mathscr{K}}(\omega, \Psi)^{-1} L_{\omega}=\left(p_{\mathscr{K}}\left(\omega_{1}, \Psi\right)^{-1} L_{\omega_{1}}, \ldots, p_{\mathscr{K}}\left(\omega_{m}, \Psi\right)^{-1} L_{\omega_{m}}\right) .
$$

Then Propositions A.8 and A.9 of [13, Appendix] amount to the following:

2.2.5. Proposition. A section $s \in \Gamma\left(\mathscr{M}_{D}, \mathscr{E}_{(\underline{k}, r)}^{D}\right)$ is rational over the extension $L$ of $E\left(\underline{k}^{D}\right)$ if and only if, for every pair $(\mathscr{K}, \Psi)$ as in Lemma 2.1 .2 and every $\gamma \in D^{\times}\left(\mathbf{A}^{f}\right)$, the restriction of the translate $\gamma^{*}(s)$ to $\operatorname{Sh}\left(H_{\mathscr{K}}, h_{\Psi}\right)$ satisfies the conditions

(i) For all $\omega \in X(\rho), p_{\mathscr{H}}(\omega, \Psi)^{-1} L_{\omega}\left(\gamma^{*}(s)\right) \in \overline{\mathbb{Q}}^{m}$;

(ii) For all $\sigma \in \mathrm{Gal}\left(\overline{\mathbb{Q}} / L \cdot E_{\Psi}\right)$,

$$
\left[p_{\mathscr{K}}(\omega, \Psi)^{-1} L_{\omega}\left(\gamma^{*}(s)\right)\right]^{\sigma}=p_{\mathscr{H}}\left(\omega^{\sigma}, \Psi\right)^{-1} L_{\omega^{\sigma}}\left(\gamma^{*}(s)\right) .
$$

Moreover, if $s \in \Gamma\left(\mathscr{M}_{D}, \mathscr{E}_{(\underline{k}, r)}^{D}\right)$ is rational over the extension $L$ then we can replace $\operatorname{Gal}\left(\overline{\mathbb{Q}} / L \cdot E_{\Psi}\right)$ in (ii) by $\operatorname{Gal}(\overline{\mathbb{Q}} / L)$, where $\operatorname{Gal}\left(\overline{\mathbb{Q}} / E\left(\underline{k}^{D}\right)\right)$ acts on the set $X(\rho)$, possibly allowing permutations of the components $X\left(\chi_{i}\right)$, and on the set of Shimura subvarieties $\operatorname{Sh}\left(H_{\mathscr{K}}, h_{\Psi}\right)$ by Deligne's theorem [4, IV].

2.2.6. Remark. As explained in $[9, \mathrm{I}, 1.3 .6]$, the truth of this proposition depends on the choice of a canonical trivialization. For the cotangent bundle $\Omega_{\mathscr{M}_{D}}^{1}$, we take the trivialization analogous to the one described in [loc.cit.], based on the cotangent vectors $d z_{j}, j \in \Sigma(D)$, to $\left(\mathfrak{H}^{ \pm}\right)^{\Sigma(D)}$ at $h_{\Psi}$. We identify $\Omega_{\mathscr{M}_{D}}^{1} \cong \bigoplus_{\sigma_{j} \in \Sigma(D)} \mathscr{\mathscr { E }}_{\left(\underline{2}_{j}, 0\right)}^{D}$, where $\underline{2}_{j}$ is the $d$-tuple with 2 in the $\sigma_{j}$ th place and zeros elsewhere. Letting $\underline{2}_{D}=\sum_{\sigma_{j} \in \Sigma(D)} \underline{2}_{j}$, we may then identify the sheaf $\Omega_{\mathscr{M}_{D}}^{\left|\sum(D)\right|}$ of top differentials with $\mathscr{E}_{\left(\underline{2}_{D}, 0\right)}^{D}$.

2.3. Automorphic forms on unitary groups. In practice, we will be looking at slightly more general groups. Let $D$ be as above, and let $(\mathscr{K}, \Psi),\left(\mathscr{K}^{\prime}, \Psi^{\prime}\right)$ be pairs as in 2.1.2; thus there exist embeddings $i, i^{\prime}: \mathscr{K}, \mathscr{K}^{\prime} \rightarrow D$ of algebras over $E$. Let

$$
G U_{\mathscr{K}}(D)=H_{\mathscr{K}} \times D^{\times} / R_{E / \mathbb{Q}} \mathbb{G}_{m, E},
$$

where $R_{E / \mathbb{Q}} \mathbb{G}_{m, E}$ is embedded diagonally. The notation will be explained in §3. The pair $\left(G U_{\mathscr{H}}(D), 1 \times\left(\mathfrak{H}^{ \pm}\right)^{\Sigma(D)}\right)$ where 1 is the trivial homomorphism to $H_{\mathscr{K}}$, satisfies the axioms for Shimura varieties; the variety

$$
\mathscr{M}_{\mathscr{K}, D}:=\operatorname{Sh}\left(G U_{\mathscr{K}}(D), 1 \times\left(\mathfrak{H}^{ \pm}\right)^{\Sigma(D)}\right)
$$

has a canonical model over $E\left(D^{\times},\left(\mathfrak{H}^{ \pm}\right)^{\Sigma(D)}\right)$, and there is an obvious morphism of canonical models:

$$
\operatorname{Sh}\left(H_{\mathscr{K}}, 1\right) \times \mathscr{M}_{D} \rightarrow \mathscr{M}_{\mathscr{K}, D} .
$$


Indeed, the factor $H_{\mathscr{K}}$ contributes nothing to the arithmetic of the canonical model, and only makes the space of connected components larger. Other Shimura varieties, involving $H_{\mathscr{K}}$ in a nontrivial way, can also be associated to $G U_{\mathscr{K}}(D)$; these varieties play an essential role in what Shimura calls the "second descent" in his construction of the canonical model of $\mathscr{M}_{D}$. These canonical models are also used in studying the special values of $L$-functions of $2 \times 2$ unitary groups in [11], and they could just as well have been used here, but the present formulation makes bookkeeping easier (however, cf. the Introduction).

There are morphisms $\operatorname{Sh}\left(H_{\mathscr{K}^{\prime}}, h_{\Psi^{\prime}}\right) \rightarrow \mathscr{M}_{D} \rightarrow \mathscr{M}_{\mathscr{K}, D}$ associated to the maps

$$
\left(H_{\mathscr{K}^{\prime}}, h_{\Psi^{\prime}}\right) \rightarrow\left(D^{\times},\left(\mathfrak{H}^{ \pm}\right)^{\Sigma(D)}\right) \rightarrow\left(G U_{\mathscr{K}}(D), 1 \times\left(\mathfrak{H}^{ \pm}\right)^{\Sigma(D)}\right)
$$

of Shimura data; here the map $D^{\times} \rightarrow G U_{\mathscr{K}}(D)$ is the obvious one. Let

$$
i_{\mathscr{K}^{\prime}}: \operatorname{Sh}\left(H_{\mathscr{K}}, 1\right) \times \operatorname{Sh}\left(H_{\mathscr{K}^{\prime}}, h_{\Psi^{\prime}}\right) \rightarrow \mathscr{M}_{\mathscr{K}, D}
$$

be the map associated to the obvious map on the first factor and (2.3.1) on the second.

A triple $(\underline{k}, \chi, r)$, where $(\underline{k}, r)$ is a $(d+1)$-tuple as above and $\chi \in X\left(H_{\mathscr{K}}\right)$, defines an automorphic vector bundle $\mathscr{E}_{(\underline{k}, r ; \chi)}^{\mathscr{K}}$ on $\mathscr{M}_{\mathscr{K}, D}$ which pulls back to $\left[V_{\chi}\right] \otimes \mathscr{E}_{(\underline{k}, r)}^{D}$ on $\operatorname{Sh}\left(H_{\mathscr{K}}, 1\right) \times \mathscr{M}_{D}$, if and only if $r=w(\chi)$. Clearly, $\mathscr{E}_{(\underline{k}, r ; \chi)}^{\mathscr{K}}$ has a canonical model over $E\left(\underline{k}^{D}\right)$. Let $\rho_{\mathscr{K}^{\prime}}=\left.\rho_{(\underline{k}, r)}^{D}\right|_{H_{K^{\prime}}} \cong \bigoplus_{i=1}^{m} \chi_{i}$, and write $X\left(\rho_{\mathscr{K}^{\prime}}\right)=X\left(\chi_{1}\right) \times \cdots \times X\left(\chi_{m}\right)$, as above. There are isomorphisms

$$
\underline{\operatorname{Lift}}_{(\underline{k}, r ; \chi)}^{\mathscr{K}}: \Gamma\left(\mathscr{M}_{\mathscr{K}, D}(\mathbb{C}), \mathscr{E}_{(\underline{k}, r ; \chi)}^{\mathscr{K}}\right) \stackrel{\sim}{\rightarrow} \mathscr{A}^{\mathscr{K}}(\underline{k}, r, \chi),
$$

where $\mathscr{A}^{\mathscr{K}}(\underline{k}, r, \chi) \cong C_{\chi} \otimes \mathscr{A}^{D}(\underline{k}, r)$, with $C_{\chi}$ as in (1.2.1), but now where $\mathscr{A}^{D}(\underline{k}, r)=\mathscr{A}^{D}\left(\underline{k}, r ; \mathscr{K}^{\prime}, \Psi^{\prime}\right)$. We let $(L \circ \mathscr{A})^{\mathscr{K}}(\underline{k}, r, \chi)$ denote the spaces of coefficients of functions in $\mathscr{A}^{\mathscr{K}}(\underline{k}, r, \chi)$, as in the remarks following (2.1.10).

Proposition 2.2.5 immediately yields the following corollary.

2.3.3. Corollary. A section $s \in \Gamma\left(\mathscr{M}_{\mathscr{K}, D^{\mathscr{E}}}^{\mathscr{E}_{(\underline{k}, r ; \chi)}^{\mathscr{K}}}\right)$ is rational over the extension $L$ of $E\left(\underline{k}^{D}\right)$ if and only if, for every $\left(\mathscr{K}^{\prime}, \Psi^{\prime}\right)$ as above and every $\gamma \in$ $G U_{\mathscr{K}}\left(D, \mathbf{A}^{f}\right)$, the pullback by $i_{\mathscr{K}^{\prime}}$ of the translate $\gamma^{*}(s)$ to $\operatorname{Sh}\left(H_{\mathscr{K}}, 1\right) \times$ $\operatorname{Sh}\left(H_{\mathscr{K}^{\prime}}, h_{\Psi^{\prime}}\right)$ satisfies the conditions

(i) For all $\left(\omega, \omega^{\prime}\right) \in X(\chi) \times X\left(\rho_{\mathscr{K}^{\prime}}\right), p_{\mathscr{K}^{\prime}}\left(\omega^{\prime}, \Psi^{\prime}\right)^{-1} L_{\omega} \otimes L_{\omega^{\prime}}\left(\gamma^{*}(s)\right) \in \overline{\mathbb{Q}}^{m}$;

(ii) For all $\sigma \in \operatorname{Gal}\left(\overline{\mathbb{Q}} / L \cdot E_{\Psi} \cdot E_{\Psi^{\prime}}\right)$,

$$
\left[p_{\mathscr{K}^{\prime}}\left(\omega^{\prime}, \Psi^{\prime}\right)^{-1} L_{\omega} \otimes L_{\omega^{\prime}}\left(\gamma^{*}(s)\right)\right]^{\sigma}=\left[p_{\mathscr{K}^{\prime}}\left(\omega^{\prime, \sigma}, \Psi^{\prime}\right)\right]^{-1} L_{\omega^{\sigma}} \otimes L_{\omega^{\prime}, \sigma}\left(\gamma^{*}(s)\right) \text {. }
$$

In (i) and (ii), the vector $p_{\mathscr{K}^{\prime}}\left(\omega^{\prime}, \Psi^{\prime}\right)^{-1} L_{\omega} \otimes L_{\omega^{\prime}}(s) \in \mathbb{C}^{m}$ is defined in analogy with the considerations preceding Proposition 2.2.5.

Moreover, if $X\left(\rho_{\mathscr{K}^{\prime}}\right)=X\left(\chi_{1}\right) \times \cdots \times X\left(\chi_{m}\right)$, as above, write $L_{\omega} \otimes L_{\omega^{\prime}}\left(\gamma^{*}(s)\right)=$ $\left(L_{\omega} \otimes L_{\omega_{j}^{\prime}}\left(\gamma^{*}(s)\right)\right), \omega_{j}^{\prime} \in X\left(\chi_{i}\right)$. Then it suffices to verify the analogues of (i), (ii) 
for $\left(L_{\omega} \otimes L_{\omega_{a}^{\prime}}\left(\gamma^{*}(s)\right)\right)$ with $\omega \in X(\chi), \omega_{a}^{\prime} \in X\left(\chi_{a}\right)$, for one fixed a (bearing in mind that $\left(\omega_{a}^{\prime}\right)^{\sigma} \in X\left(\chi_{b}\right)$ where possibly $\left.\chi_{b} \neq \chi_{a}\right)$.

Proof. Everything is obvious except the final assertion. We treat the analogous assertion for $D^{\times}$; the case of $G U$ is identical. Thus we place ourselves in the situation of Proposition 2.2.5. Suppose $L_{\omega_{i}}\left(\gamma^{*}(s)\right)$ satisfies (i) and (ii) for all $\omega_{i} \in X\left(\chi_{i}\right)$. Now $\underline{\operatorname{Lift}}_{(\underline{k}, r)}^{D}\left(\gamma^{*}(s)\right)$ is an $L_{(\underline{k}, r)}^{D}$-valued function on $D^{\times}(\mathbb{Q}) \backslash D^{\times}(\mathbf{A})$ which satisfies the automorphy condition (2.1.8). Write $L_{(\underline{k}, r)}^{D}=\bigoplus_{i=1}^{m} L_{(\underline{k}, r)}^{D}\left(\chi_{i}\right)$. Fix $\sigma \in \operatorname{Gal}\left(\overline{\mathbb{Q}} / L \cdot E_{\Psi^{\prime}}\right)$. Under our hypothesis, the $L_{(\underline{k}, r)}^{D}\left(\chi_{a}\right)$-component of $\underline{\operatorname{Lift}}_{(\underline{k}, r)}^{D}\left(\left(s-s^{\sigma}\right)\right)$ is identically zero (by density of $D^{\times}\left(\mathbf{A}^{f}\right)$-orbits in $\left.D^{\times}(\mathbb{Q}) \backslash D^{\times}(\mathbf{A})\right)$. But $L_{(\underline{k}, r)}^{D}$ is an irreducible $Z_{D^{\times}}(\mathbb{R}) \cdot K_{\infty}^{+}$module, so the automorphy condition implies that $\underline{\operatorname{Lift}}_{(\underline{k}, r)}^{D}\left(\left(s-s^{\sigma}\right)\right)$ is identically zero. Thus $s=s^{\sigma}$.

2.3.4. Remark. The distinction between the coefficients of $\mathscr{A}^{D}(\underline{k}, r)$, and the elements of $\mathscr{A}^{D}(\underline{k}, r)$, which are vector-valued automorphic forms, complicates the notation considerably, but is otherwise of no significance. Here is how to pass from one to the other; a similar procedure can be applied to $\mathscr{A}^{\mathscr{K}}(\underline{k}, r, \chi)$. Let $\left\{v\left(\chi_{i}\right), i=1, \ldots, m\right\}$ be a basis of $H_{\mathscr{K}^{-}}$ eigenfunctions of $L_{(\underline{k}, r)}^{D}(\overline{\mathbb{Q}})$ with the property that $v\left(\chi_{i}\right)^{\sigma}=v\left(\chi_{i}^{\sigma}\right)$ for all $\sigma \in$ $\operatorname{Gal}\left(\overline{\mathbb{Q}} / E\left(\underline{k}^{D}\right) \cdot E_{\Psi}\right)$. Note that each $\chi_{i}$ has multiplicity one in $L_{(\underline{k}, r)}^{D}$. The $\left\{v\left(\chi_{i}\right)\right\}$ define a canonical trivialization of $\mathscr{L}_{(\underline{k}, r)}^{D}$, or of $\mathscr{E}_{(\underline{k}, r)}^{D}$, near the point $h_{\Psi}$. Let $\Gamma\left(\mathscr{M}_{D}(\mathbb{C}), \mathscr{E}_{(\underline{k}, r)}^{D}\right)^{\pi}$, for some $\pi$, and let $\underline{f}=\sum f_{i} \cdot v\left(\chi_{i}\right)=\underline{\operatorname{Lift}}(s) \in$ $\mathscr{A}^{D}(\underline{k}, r)$. If $\omega \in X\left(\chi_{i}\right)$, then we may write $L_{\omega}(s)=L_{\omega}\left(f_{i}\right)$, where the latter is defined by the integral (1.2.2). But $f_{i}$ belongs to the automorphic representation $\pi^{D}$. In this way, using a canonical trivialization as above, we can translate the arithmeticity criterion of 2.2.5 and 2.3.2 into a statement about holomorphic elements of $\pi^{D}$, which can then be tested directly. We do this with minimal further comment in what follows.

2.4. Complex conjugation. Henceforward, we restrict attention to the case $\mathscr{K}^{\prime}$ $=\mathscr{K}$. Thus the rationality criterion of Proposition 2.3.3 is only verified for fields containing $E\left(\underline{k}^{D}\right) \cdot E_{\Psi}$. In order to carry out a "second descent" for automorphic forms on $D^{\times}$, we can let the group $G U_{\mathscr{K}}(D)$ vary; however, this will not be done in this paper. Choose a main involution $l=l_{\mathscr{K}^{\prime}}$ of $D$ which induces the nontrivial element, also denoted $l$ in $\S 1$, of $\operatorname{Gal}\left(\mathscr{K}^{\prime} / E\right)$ on $\mathscr{K}^{\prime}$. Then $i^{\prime}$ makes $D$ into a $\mathscr{K}^{\prime}$-vector space of dimension 2. Moreover, $i^{\prime}\left(\mathscr{K}^{\prime, \times}\right)$ is of index 2 in its normalizer $N_{D^{\times}}\left(i\left(\mathscr{K}^{\prime, \times}\right)\right)$ in $D^{\times}$, and we may choose an element $\delta=\delta_{\mathscr{K}^{\prime}} \in N_{D^{\times}}\left(i^{\prime}\left(\mathscr{K}^{\prime, \times}\right)\right)-i^{\prime}\left(\mathscr{K}^{\prime}, \times\right)$ such that $\delta^{2} \in E^{\times}$, such that conjugation by $\delta$ induces $l$ on $\mathscr{K}^{\prime}$, and such that $\delta^{l}=-\delta$. Let $e(\mathscr{K})=\delta^{2} \in E^{\times}$.

Let $\delta_{\infty} \in D^{\times}(\mathbb{R})$ be the archimedean component of $\delta$, and let $\delta_{f}$ be its 
finite adelic component, so that $\delta=\delta_{\infty} \cdot \delta_{f}$ as elements of $D^{\times}(\mathbf{A})$. For any function $\varphi$ on $D^{\times}(\mathbf{A})$, let $\varphi^{\mathscr{f}(\mathscr{K})}(g)=\varphi\left(g \cdot \delta_{\infty}\right)$; for $\underline{k}, r$ as above, let

$$
\mathscr{A}^{D}(\underline{k}, r)^{\Sigma(D)}=\left\{\varphi^{\mathscr{J}(\mathscr{K})}, \varphi \in \mathscr{A}^{D}(\underline{k}, r)\right\} \text {. }
$$

Then the space $\mathscr{A}^{D}(\underline{k}, r)^{\Sigma(D)}$ does not depend on the choice of $\delta$ and, for any $F \in \mathscr{A}^{D}(\underline{k}, r)$, the complex conjugate $\bar{F} \in \mathscr{A}^{D}(\underline{k}, r)^{\Sigma(D)}$, just as in [9, I, $\S 1]$. Thus the map $F \mapsto \mathscr{J}(F):=\bar{F}^{\mathscr{F}(\mathscr{K})}$ is a complex antilinear involution of $\mathscr{A}^{D}(\underline{k}, r)$.

On the other hand, complex conjugation $l \in \mathrm{Gal}(\overline{\mathbb{Q}} / \mathbb{Q})$ preserves $\mathscr{M}_{D}$ and $\mathscr{E}_{(\underline{k}, r)}^{D}$, hence acts semilinearly on $\Gamma\left(\mathscr{M}_{D}, \mathscr{E}_{(\underline{k}, r)}^{D}\right)$. Let $\pi$ be a cuspidal automorphic representation of $D^{\times}$. Evidently

$$
l\left(\Gamma\left(\mathscr{M}_{D}, \mathscr{E}_{(\underline{k}, r)}^{D}\right)^{\pi}\right)=\mathscr{J}\left(\Gamma\left(\mathscr{M}_{D}, \mathscr{E}_{(\underline{k}, r)}^{D}\right)^{\pi}\right)=\Gamma\left(\mathscr{M}_{D}, \mathscr{E}_{(\underline{k}, r)}^{D}\right)^{\pi^{l}},
$$

where $\pi^{l}$ is the conjugate of $\pi$, as in the discussion preceding Lemma 1.4.6 of $[9, I]$. Denote again by $l$ the corresponding complex antilinear involution of $\mathscr{A}^{D}(\underline{k}, r)$.

We want to compare $l$ and $\mathscr{J}$. In the case of $G L(2)$, Shimura observed that they can be more or less identified, at least in their actions on new vectors; cf. [9, I, Lemma 1.4.6] for an account in the present language. In general, there is no theory of new vectors, because of the finite places at which $D$ ramifies. As a substitute, we use the fact that characters of $\mathscr{K}_{v}^{\times}$occur with multiplicity one in $\pi_{v}^{D}$ [33, Remark 3.5]. Thus, define $S_{f}(D)$ as in the proof of Proposition 2.2.4, and, for each $v \in S(D):=\Sigma(D)^{\prime} \amalg S_{f}(D)$, let $\chi_{v}$ be a character of $\mathscr{K}_{v}^{\times}$which occurs in the restriction of $\pi_{v}^{D}$. Note that $\mathscr{K}_{v}$ is necessarily a quadratic field extension of $E_{v}$ for $v \in S(D)$. Let $\delta_{S_{f}(D)} \in \prod_{v \in S_{f}(D)} D_{v}^{\times}$be the $S_{f}(D)$-component of $\delta$, viewed as an element of $D^{\times}(\mathbf{A})$, and similarly, let $\delta_{D}=\delta_{\infty} \cdot \delta_{S_{f}(D)}$. Let $(L \circ \mathscr{A})^{D}(\underline{k}, r)^{\pi}=\pi^{D} \cap(L \circ \mathscr{A})^{D}(\underline{k}, r)$ (the holomorphic vectors in $\left.\pi^{D}\right)$, and let $(L \circ \mathscr{A})^{D}(\underline{k}, r)^{\pi}\left(\left\{\chi_{v}\right\}\right) \subset \pi^{D}$ be the $\bigotimes_{v \in S(D)} \chi_{v}$-isotypic subspace of $(L \circ \mathscr{A})^{D}(\underline{k}, r)$ for $\prod_{v \in S(D)} \mathscr{K}_{v}^{\times}$. Finally, let $\pi^{D}\left(\left\{\chi_{v}\right\}\right)^{\text {new }}$ be the fixed space under the compact open subgroup $K_{f}\left(\pi^{D}\right)$ defined in the proof of Proposition 2.2.4. Then $\pi^{D}\left(\left\{\chi_{v}\right\}\right)^{\text {new }}$ is one-dimensional, and the map $F \mapsto$ $\bar{F}\left(\cdot \delta_{D}\right)$ defines an antilinear isomorphism

$$
\overline{\mathscr{J}}\left(\left\{\chi_{v}\right\}\right): \pi^{D}\left(\left\{\chi_{v}\right\}\right)^{\text {new }} \stackrel{\sim}{\rightarrow} \pi^{l, D}\left(\left\{\bar{\chi}_{v}^{l}\right\}\right)^{\text {new }} .
$$

Let $\left\{\chi_{v}\right\}_{\infty} \in X\left(\rho_{(\underline{k}, r)}^{D}\right)$ be the character corresponding to the $\chi_{v}$ with $v \in$ $\Sigma(D)^{\prime}$. Let $E\left(\pi^{D},\left\{\chi_{v}\right\}\right)$ denote the extension of $\mathbb{Q}\left(\pi^{D}\right) \cdot E\left(\left\{\chi_{v}\right\}_{\infty}\right)$ obtained by adjoining the values of $\left\{\chi_{v}\right\}$ for $v \in S_{f}(D)$; since $\chi_{v}$ occurs in $\pi_{v}^{D}$, this is clearly a CM field (cf. the remark following (2.2.2)). Then $E\left(\pi^{l, D},\left\{\bar{\chi}_{v}^{l}\right\}\right)=$ $E\left(\pi^{D},\left\{\bar{\chi}_{v}\right\}\right)^{l}=E\left(\pi^{D},\left\{\bar{\chi}_{v}\right\}\right)=E\left(\pi^{D},\left\{\chi_{v}\right\}\right)$, and both sides of (2.4.2) have 
natural $E\left(\pi^{D},\left\{\chi_{v}\right\}\right)$-rational structures, with the property that

$$
\pi^{D}\left(\left\{\chi_{v}\right\}\right)^{\text {new }}\left(E\left(\pi^{D},\left\{\chi_{v}\right\}\right)\right)^{\sigma}=\pi^{D}\left(\left\{\chi_{v}^{\sigma}\right\}\right)^{\text {new }}\left(E\left(\pi^{D},\left\{\chi_{v}^{\sigma}\right\}\right)\right)
$$

for all $\sigma \in \operatorname{Gal}\left(\overline{\mathbb{Q}} / \mathbb{Q}\left(\pi^{D}\right) \cdot E_{\Psi}\right)$. Indeed, the comparable statement without the conditions at finite primes has already been observed in Remark 2.3.4 (cf. 2.5.7, below), and the action of $D^{\times}\left(\mathbf{A}^{f}\right)$ is rational over the field $E\left(\underline{k}^{D}\right) \subset E\left(\pi^{D}\right)$. Let $c\left(\left\{\chi_{v}\right\}\right) \in \mathbb{C}^{\times}$be a constant such that

$$
\begin{aligned}
& \overline{\mathscr{J}}\left(\left\{\chi_{v}\right\}\right)\left(\pi^{D}\left(\left\{\chi_{v}\right\}\right)\right)^{\text {new }}\left(E\left(\pi^{D},\left\{\chi_{v}\right\}\right)\right) \\
& \quad=c\left(\left\{\chi_{v}\right\}\right) \cdot \pi^{l, D}\left(\left\{\overline{\chi_{v}^{l}}\right\}\right)^{\text {new }}\left(E\left(\pi^{D},\left\{\chi_{v}\right\}\right)\right) .
\end{aligned}
$$

The following lemma is a more precise version of [40, II, Lemma 4.2], and is a generalization to automorphic vector bundles of [27, II, Theorem 7.2].

2.4.5. Lemma. The $c\left(\left\{\chi_{v}\right\}\right)$ can be chosen so that $c\left(\left\{\chi_{v}\right\}\right)^{\sigma}=c\left(\left\{\chi_{v}^{\sigma}\right\}\right)$ for all $\sigma \in \operatorname{Aut}\left(\mathbb{C} / \mathbb{Q}\left(\pi^{D}\right) \cdot E_{\Psi}\right)$. In particular, $c\left(\left\{\chi_{v}\right\}\right) \in E\left(\pi^{D},\left\{\chi_{v}\right\}\right)^{\times}$.

Proof. It would be preferable to have a more precise identification of the action of complex conjugation on automorphic vector bundles, but (for reasons having to do with the nonuniqueness of $\delta$ ) this may be the best possible. We imitate Shimura's proof in [40], using the rationality criterion of Proposition 2.2.5. Suppose $F \in \pi^{D}\left(\left\{\chi_{v}\right\}\right)^{\text {new }}\left(E\left(\pi^{D},\left\{\chi_{v}\right\}\right)\right)$. It suffices to verify that $\bar{J}\left(\left\{\chi_{v}\right\}\right)(F)^{\sigma}=\overline{\mathscr{J}}\left(\left\{\chi_{v}^{\sigma}\right\}\right)(F)$ for all $\sigma \in \operatorname{Aut}\left(\mathbb{C} / \mathbb{Q}\left(\pi^{D}\right) \cdot E_{\Psi}\right)$. We compute $L_{\omega}\left(\bar{J}\left(\left\{\chi_{v}\right\}\right)\left(\gamma^{*}(F)\right)\right)$, where $\omega$ is a Hecke character of $\mathscr{K}$ with $\xi_{\omega} \cdot \xi_{\pi}=1$ and $\gamma \in D^{\times}\left(\mathbf{A}^{f}\right)$ (here we abuse notation by confusing $F$ with the section of an automorphic vector bundle defined by $F$ ). It has to be shown that

$$
\left[p_{\mathscr{K}}\left(\overline{\omega^{l}}, \Psi\right)^{-1} L_{\omega}\left(\overline{\mathscr{J}}\left(\left\{\chi_{v}\right\}\right)\left(\gamma^{*}(F)\right)\right)\right]^{\sigma}=p_{\mathscr{K}}\left(\left(\bar{\omega}^{\sigma}\right)^{l}, \Psi\right)^{-1} L_{\omega}\left(\overline{\mathscr{J}}\left(\left\{\chi_{v}^{\sigma}\right\}\right)\left(\gamma^{*}(F)\right)\right),
$$

for all $\sigma \in \operatorname{Aut}\left(\mathbb{C} / \mathbb{Q}\left(\pi^{D}\right) \cdot E_{\Psi}\right)$ and all $\omega \in X(\chi)$. Now

$$
\begin{aligned}
L_{\omega}(\overline{\mathcal{J}} & \left.\left(\left\{\chi_{v}\right\}\right)\left(\gamma^{*}(F)\right)\right)=L_{\omega}\left(\gamma^{*}(\bar{F})\left(\cdot \delta_{D}\right)\right) \\
& =\int_{H(\mathbb{Q}) \cdot H(\mathbb{R}) \backslash H(\mathbf{A})} \bar{F}\left(t \cdot \delta_{D} \cdot \gamma\right) \cdot \omega(t) d t \quad\left(H=H_{\mathscr{K}}\right) \\
& =\int_{H(\mathbb{Q}) \cdot H(\mathbb{R}) \backslash H(\mathbf{A})} \bar{F}\left(\delta^{-1} \cdot t^{l} \cdot \gamma^{\prime}\right) \cdot \omega(t) d t, \quad \text { where } \gamma^{\prime}=\delta_{D}^{-1} \cdot \delta \cdot \gamma \in D^{\times}\left(\mathbf{A}^{f}\right) \\
& =\int_{H(\mathbb{Q}) \cdot H(\mathbb{R}) \backslash H(\mathbf{A})} \bar{F}\left(t^{l} \cdot \gamma^{\prime}\right) \cdot \omega(t) d t \quad\left(\text { since } \delta^{-1} \in D^{\times}(\mathbf{Q})\right) \\
& =\int_{H(\mathbb{Q}) \cdot H(\mathbb{R}) \backslash H(\mathbf{A})} \bar{F}\left(t \cdot \gamma^{\prime}\right) \cdot \omega^{l}(t) d t=\overline{L-\left(\gamma^{\prime}, *(F)\right)} .
\end{aligned}
$$

But it is shown in the appendix (A.1) that we may take

$$
p_{\mathscr{K}}\left(\overline{\omega^{l}}, \Psi\right) \sim_{E_{\Psi} \cdot E(\omega)} \overline{p_{\mathscr{K}}(\omega, \Psi)} .
$$

This proves $(2.4 .5 .1)$, at least for $\sigma \in \mathrm{Gal}\left(\overline{\mathbb{Q}} / \mathbb{Q}\left(\pi^{D}\right) \cdot E(\omega)\right)$, and the descent to $\mathbb{Q}\left(\pi^{D}\right) \cdot E_{\Psi}$ follows similarly from (A.2). 
2.4.6. Remark. A special case of this lemma is used implicitly in $\S 15$ of [13], and should have been proved there.

2.4.7. It follows that $l \circ \mathcal{J}$ defines a $\mathbb{Q}\left(\pi^{D}\right) \cdot E_{\Psi}$-rational automorphism of $\Gamma\left(\mathscr{M}_{D}, \mathscr{E}_{(\underline{k}, r)}^{D}\right)^{\pi}$. The case of $G L(2)$ used in the appendix relies heavily on the theory of the $q$-expansion.

2.5. Quadratic periods. We now introduce the basic quadratic period of the automorphic representation $\pi^{D}$. Following [9, I, (1.4.3.2-3)], we may associate to $\varphi \in \mathscr{A}^{D}(\underline{k}, r)^{\Sigma(D)}$ a $(0,|\Sigma(D)|)$-form $\omega_{\varphi}$ on $\mathscr{M}_{D}$ with coefficients in $\mathscr{E}_{(\underline{k}(\Sigma(D)), r)}^{D}$. Here $\underline{k}(\Sigma(D))$ is the $d$-tuple such that $k(\Sigma(D))_{j}=k_{j}$ if $j \notin \Sigma(D)$, $k(\Sigma(D))_{j}=2-k_{j}$ is $j \in \Sigma(D)$. Now (2.2.6) defines a homomorphism

$$
\mathscr{E}_{(\underline{k}, r)}^{D} \otimes \mathscr{E}_{(\underline{k}(\Sigma(D)),-r)}^{D} \rightarrow \mathscr{E}_{\left(\underline{2}_{D}, 0\right)}^{D} \cong \Omega_{\mathscr{M}_{D}}^{|\Sigma(D)|}
$$

In fact,

$$
L_{(\underline{k}, r)}^{D} \otimes L_{(\underline{k}(\Sigma(D)),-r)}^{D} \cong L_{\left(\underline{2}_{D}, 0\right)}^{D} \otimes \prod_{j \in \Sigma(D)^{\prime}} \pi^{\mathbb{H}}\left(k_{j}\right)^{\vee} \otimes \prod_{j \in \Sigma(D)^{\prime}} \pi^{\mathbb{H}}\left(k_{j}\right)
$$

as $K_{\infty}^{+}$-module, and contraction of the last two factors defines the first arrow in (2.5.1). If $\varphi \in \mathscr{A}^{D}(\underline{k}, r)^{\Sigma(D)}$, let

$$
\varphi(-r)(g)=(2 \pi i)^{-r|\Sigma(D)|} \varphi(g) \cdot\left\|N_{D}(g)\right\|_{\mathbf{A}}^{-r} \in \mathscr{A}^{D}(\underline{k},-r)^{\Sigma(D)} .
$$

Then

$$
\varphi \smile \varphi^{\prime} \underset{\operatorname{def}}{=}(2 \pi i)^{-|\Sigma(D)|} \int_{\mathscr{M}_{D}}\left[\omega_{\varphi} \wedge \omega_{\varphi^{\prime}(-r)}\right],
$$

where $[*]$ denotes contraction, is a well-defined pairing

$$
\bar{H}^{0}\left(\mathscr{E}_{(\underline{k}, r)}^{D}\right) \otimes \bar{H}^{|\Sigma(D)|}\left(\mathscr{E}_{(\underline{k}(\Sigma(D)), r)}^{D}\right) \rightarrow \mathbb{C} .
$$

Here the subspaces $\bar{H}^{q}\left(\mathscr{E}_{(\underline{k}, r)}^{D}\right) \subset H^{q}\left(\mathscr{E}_{(\underline{k}, r)}^{D}\right)$ are defined as in [9, I] when $D$ is split, and $\bar{H}^{q}\left(\mathscr{E}_{(\underline{k}, r)}^{D}\right)=H^{q}\left(\mathscr{E}_{(\underline{k}, r)}^{D}\right)$ when $D$ is anisotropic.

If $F, G \in \mathscr{A}^{D}(\underline{k}, r)$, we define the normalized Petersson inner product $\langle F, G\rangle_{D}=F \smile \bar{G}$. Then

(2.5.4) $\langle F \otimes \xi, G \otimes \xi\rangle_{D}=\langle F, G\rangle_{D}$ for any character $\xi$ of $D^{\times}(\mathbf{A}) / D^{\times}$.

We identify $\Gamma\left(\mathscr{M}_{D}, \mathscr{E}_{(\underline{k}, r)}^{D}\right)^{\pi}$ with the space of holomorphic forms in (the space of) $\pi^{D}$, and denote $\Gamma\left(\mathscr{M}_{D}, \mathscr{E}_{(\underline{k}, r)}^{D}\right)^{\pi}(\mathbb{Q}(\pi, D))$ the $\mathbb{Q}(\pi, D)$-rational structure constructed in 2.2.4.

2.5.5. Lemma. For each $D$ such that $\pi^{D}$ exists, there is a constant $q^{D}(\pi) \in \mathbb{C}^{\times}$ such that, for every pair $\varphi, \varphi^{\prime} \in \Gamma\left(\mathscr{M}_{D}, \mathscr{E}_{(\underline{k}, r)}^{D}\right)^{\pi}(\mathbb{Q}(\pi, D))$,

$$
\left\langle\varphi, \varphi^{\prime}\right\rangle_{D} \sim_{\mathbb{Q}(\pi, D) ; \mathbb{Q}} q^{D}(\pi) .
$$


Moreover, if $\varphi^{\prime} \in \Gamma\left(\mathscr{M}_{D}, \mathscr{E}_{\underline{\underline{k}}, r)}^{D}\right)^{\pi}$ satisfies

$$
q^{D}(\pi)^{-1} \cdot\left\langle\varphi, \varphi^{\prime}\right\rangle_{D} \in \mathbb{Q}(\pi, D) \text { for all } \varphi \in \Gamma\left(\mathscr{M}_{D}, \mathscr{E}_{(\underline{k}, r)}^{D}\right)^{\pi}(\mathbb{Q}(\pi, D)),
$$

then $\varphi^{\prime} \in \Gamma\left(\mathscr{M}_{D}, \mathscr{E}_{(\underline{k}, r)}^{D}\right)^{\pi}(\mathbb{Q}(\pi, D))$.

Proof. The first statement follows exactly as in [13, §15], except for the $\operatorname{Gal}(\overline{\mathbb{Q}} / \mathbb{Q})$-equivariance; the power of $(2 \pi i)$ comes from the definition of $\varphi(-r)$. But one can tautologically choose the $q\left(\pi^{\tau}, D^{(\tau)}\right)$ consistently so that, for all $\tau \in \operatorname{Gal}(\overline{\mathbb{Q}} / \mathbb{Q})$,

$$
\left[q^{D}(\pi)^{-1} \cdot\left\langle\varphi, \varphi^{\prime}\right\rangle_{D}\right]^{\tau}=q^{D^{(\tau)}}\left(\pi^{\tau}\right)^{-1} \cdot\left\langle\varphi^{\tau}, \varphi^{\prime, \tau}\right\rangle_{D}
$$

The second statement is obvious.

It is practical to translate this into a criterion for rationality of the coefficients of vector-valued forms, which are elements of the automorphic representation $\pi^{D}$. Let $\varphi, \varphi^{\prime} \in \Gamma\left(\mathscr{M}_{D}, \mathscr{E}_{(\underline{k}, r)}^{D}\right)^{\pi}$, and write $\underline{\operatorname{Lift}}(\varphi)=\sum \varphi_{i} \cdot v\left(\chi_{i}\right) \in$ $\mathscr{A}^{D}(\underline{k}, r, K, \Psi)$, in the notation of Remark 2.3.4, $\underline{\operatorname{Lift}}\left(\varphi^{\prime}\right)=\sum \varphi_{i}^{\prime} \cdot v\left(\chi_{i}\right)$. Let $(\cdot, \cdot)$ denote the $L^{2}$-inner product on $(L \circ \mathscr{A})^{D}(\underline{k}, r, \mathscr{K}, \Psi)$. We write $\varphi_{i}(\mathscr{K}, \Psi)=\varphi_{i}$ to emphasize dependence on the chosen point of trivialization.

2.5.6. Corollary. For every pair $\varphi, \varphi^{\prime} \in \Gamma\left(\mathscr{M}_{D}, \mathscr{E}_{(\underline{k}, r)}^{D}\right)^{\pi}(\mathbb{Q}(\pi, D))$ as above,

$$
\left(\varphi_{i}(\mathscr{K}, \Psi), \varphi_{i}^{\prime}(\mathscr{K}, \Psi)\right) \sim_{\mathbb{Q}(\pi, D) \cdot E\left(\chi_{i}\right) ; \mathbb{Q}} q^{D}(\pi)
$$

where it is understood that $\mathrm{Gal}(\overline{\mathbb{Q}} / \mathbb{Q})$ permutes the $\chi_{i}$ and the CM pairs $(\mathscr{K}, \Psi)$ and thus permutes the $\varphi_{i}(\mathscr{K}, \Psi)$. Moreover, if $\varphi^{\prime} \in \Gamma\left(\mathscr{M}_{D}, \mathscr{E}_{(\underline{k}, r)}^{D}\right)^{\pi}$ satisfies $\left(\varphi_{i}(\mathscr{K}, \Psi), \varphi_{i}^{\prime}(\mathscr{K}, \Psi)\right) \sim_{\mathbb{Q}(\pi, D) \cdot E\left(\chi_{i}\right) ; \mathbb{Q}(\pi, D)} q^{D}(\pi)$ for all $\varphi \in$ $\Gamma\left(\mathscr{M}_{D}, \mathscr{E}_{(\underline{k}, r)}^{D}\right)^{\pi}(\mathbb{Q}(\pi, D))$ then $\varphi^{\prime} \in \Gamma\left(\mathscr{M}_{D}, \mathscr{E}_{(\underline{k}, r)}^{D}\right)^{\pi}(\mathbb{Q}(\pi, D))$.

Proof. The space of coefficients of $\Gamma\left(\mathscr{M}_{D}, \mathscr{E}_{(\underline{k}, r)}\right)^{\pi}(\mathbb{Q}(\pi, D))$ defines a $D^{\times}\left(\mathbf{A}^{f}\right)$ invariant $\mathbb{Q}(\pi, D) \cdot E_{\Psi}$-rational structure on $\left(L_{(\underline{k}, r)}^{D}\right)^{*} \otimes \pi_{f}^{D}$. Each $\varphi_{i}(\mathscr{K}, \Psi)$ is an $\mathbb{Q}(\pi, D) \cdot E_{\Psi} \cdot E\left(\chi_{i}\right)$-rational vector such that $\varphi_{i}(\mathscr{K}, \Psi)^{\tau}=\varphi_{\tau(i)}(\mathscr{K}, \Psi)$ for $\tau \in \operatorname{Gal}\left(\overline{\mathbb{Q}} / \mathbb{Q}(\pi, D) \cdot E_{\Psi}\right)$, where $\tau(i)$ is defined in the obvious way. Furthermore, $(\cdot, \cdot)$ is a $D^{\times}\left(\mathbf{A}^{f}\right)$-invariant hermitian or skew-hermitian form on $\left(L_{(\underline{k}, r)}^{D}\right)^{*} \otimes \pi_{f}^{D}$. The argument in $[13, \S 15]$ thus shows the existence of a constant $q(\pi, D ; \mathscr{K}, \Psi)$ such that

$$
\left(\varphi_{i}(\mathscr{K}, \Psi), \varphi_{i}^{\prime}(\mathscr{K}, \Psi)\right) \sim_{\mathbb{Q}(\pi, D) \cdot E\left(\chi_{i}\right) ; \mathbb{Q}(\pi, D) \cdot E_{\Psi}} q(\pi, D ; \mathscr{K}, \Psi)
$$

Expressing $\left\langle\varphi, \varphi^{\prime}\right\rangle_{D}$ in terms of $(\cdot, \cdot)$, it follows from Lemma 2.5.5 that

$$
q(\pi, D ; \mathscr{K}, \Psi) \sim_{\mathbb{Q}(\pi, D) \cdot E_{\Psi}} q^{D}(\pi) .
$$

Here we are making use of the fact that $\left(\varphi_{i}(\mathscr{K}, \Psi), \varphi_{j}^{\prime}(\mathscr{K}, \Psi)\right)=0$ for $i \neq j$, because the characters $\chi_{i}$ have multiplicity one in $L_{(\underline{k}, r)}^{D}$. The $\operatorname{Gal}(\overline{\mathbb{Q}} / \mathbb{Q})$ equivariance and the second statement follow as in the proof of the lemma. 
The following statement can be extracted from the proof of the above corollary.

2.5.7. Lemma. Let $X\left(\rho_{(\underline{k}, r)}^{D}\right)=\left\{\chi_{1}, \ldots, \chi_{m}\right\}$. Let $\varphi \in \Gamma\left(\mathscr{M}_{D}, \mathscr{E}_{(\underline{k}, r)}^{D}\right)^{\pi}(\mathbb{Q}(\pi, D))$ and, for each $i$, let $\varphi_{i}(\mathscr{K}, \Psi) \in \pi^{D}$ be the corresponding coefficient of $\varphi$, so that

$$
\varphi_{i}(\mathscr{K}, \Psi) \in \pi_{f}^{D, \chi_{i}}:=v\left(\chi_{i}\right)\left(\left(L_{(\underline{k}, r)}^{D}\right)^{*}\right) \otimes \pi_{f}^{D} \subset \pi^{D} .
$$

Then the $D^{\times}\left(\mathbf{A}^{f}\right)$-translates of $\varphi_{i}$ generate a $\mathbb{Q}(\pi, D) \cdot E_{\Psi} \cdot E\left(\chi_{i}\right)$-rational structure $\pi_{f}^{D, \chi_{i}}\left(\mathbb{Q}(\pi, D) \cdot E_{\Psi} \cdot E\left(\chi_{i}\right)\right)$ on $\pi_{f}^{D, \chi_{i}} \cong \pi_{f}^{D}$.

Finally, we observe the following normalization:

2.5.8. Lemma. If $\Sigma(D)=\varnothing$; then we may take $q^{D}(\pi)=1$.

Proof. If $\Sigma(D)=\varnothing$, then $E\left(D^{\times},\left(\mathfrak{H}^{ \pm}\right)^{\Sigma(D)}\right)=\mathbb{Q}, E\left(\underline{k}^{D}\right)=E(\underline{k})$, and $\mathscr{M}_{D}$ is a profinite scheme over $\mathbb{Q}$. The space $\underline{\operatorname{Lift}}\left(\Gamma\left(\mathscr{M}_{D}, \mathscr{E}_{(\underline{k}, r)}^{D}\right)(E(\underline{k}))\right)$ may then be identified, by restriction, with the space of $\left(L_{(\underline{k}, r)}^{D}(E(\underline{k}))^{*}\right)$-valued functions on $\{1\}_{\infty} \times D^{\times}\left(\mathbf{A}^{f}\right)$, where $\{1\}_{\infty}$ is the identity in $D^{\times}(\mathbb{R})$. Since $E(\underline{k}) \subset \mathbb{Q}(\pi)$, $\underline{\operatorname{Lift}}\left(\Gamma\left(\mathscr{M}_{D}, \mathscr{E}_{(\underline{k}, r)}^{D}\right)^{\pi}(\mathbb{Q}(\pi))\right)$ is a space of $\left(L_{(\underline{k}, r)}^{D}(\mathbb{Q}(\pi))^{*}\right)$-valued functions on $\{1\}_{\infty} \times D^{\times}\left(\mathbf{A}^{f}\right)$. Since the volume of $\mathscr{M}_{D}$ is a rational number, the lemma is thus clear.

2.6. Statement of the main theorem. The following theorem is a partial answer to a conjecture of Shimura in [42, 43].

2.6.1. Theorem. Let $\pi$ be an irreducible, cuspidal, motivic automorphic representation of $G L(2, E)$, associated to automorphic forms of weight $(\underline{k}, r)$, with $k_{j} \geq 2$ for all $j$. Suppose there is a finite place $v_{0}$ of $E$ such that the local factor $\pi_{v_{0}}$ of $\pi$ is special or supercuspidal. Let $I$ and $J \subset \Sigma$, and let $D_{1}$, $D_{2}, D_{3}$, and $D_{4}$ be quaternion algebras over $E$ with $\Sigma\left(D_{1}\right)=I, \Sigma\left(D_{2}\right)=J$, $\Sigma\left(D_{3}\right)=I \cap J, \Sigma\left(D_{4}\right)=I \cup J$, and such that $\pi^{D_{i}}$ exists for $i=1,2,3,4$. Then

$$
q^{D_{1}}(\pi) \cdot q^{D_{2}}(\pi) \sim_{\overline{\mathbb{Q}}} q^{D_{3}}(\pi) \cdot q^{D_{4}}(\pi) .
$$

2.6.2. Remarks. (a) As stated, this is not the most general theorem which can be proved by the methods of this paper. For example, it will be shown in $\S 7$ that, if $d,|I|,|J|$, and $|I \cap J|$ are all even, then the local hypothesis at $v_{0}$ is unnecessary (cf. Theorem 2.6.5, below). Furthermore, the case in which $I \cap J=\varnothing, I \cup J=\Sigma$ was proved by Shimura in [42, Theorem 5.4]. However, Shimura's conjecture is more general in an essential way: he predicts the existence of invariants which play the role of the $q^{D}(\pi)$ even when $\pi^{D}$ does not exist. We have suggested elsewhere that the invariants denoted $\nu^{I}(\pi)$ in $\S 1.8$ should satisfy Shimura's conditions, and in particular have the multiplicativity property asserted in the Theorem for the $q^{D(I)}(\pi)$. A proof that this is the case will appear in $[9$, II $]$. 
The case $I \cap J=\varnothing, I \cup J=\Sigma$ will be referred to as Shimura's case of Theorem 2.6.1, and will be used in the induction step of the proof. Another proof of this case, independent of Shimura's theorem, is given in $\S 7.3$.

(b) The local conditions ensure that, for every subset $I \subset \Sigma$, there exists at least one quaternion algebra $D(I)$ with $\Sigma(D(I))=I$, such that $\pi^{D(I)}$ exists. Indeed, letting $S=I \cup\left\{v_{0}\right\}, \pi$ is in the discrete series at all places in $S$. Thus, if $D$ is any quaternion algebra over $E$ unramified outside $S, \pi^{D}$ exists. There exists exactly one quaternion algebra $D(I)$ ramified at $\Sigma-I$ and unramified outside $S$. We use this notation in the sequel.

(c) Shimura has proved [42, Theorem 5.6] that, if $D$ and $D^{\prime}$ are quaternion algebras over $E$ with $\Sigma(D)=\Sigma\left(D^{\prime}\right)$, such that $\pi^{D}$ and $\pi^{D^{\prime}}$ exist, then, under the hypotheses of Theorem 2.6.1, $q^{D}(\pi) \sim q^{D^{\prime}}(\pi)$. In fact, Shimura's statement is slightly different. His argument proves the following: if there exists a quaternion algebra $D^{\prime \prime}$ with $\Sigma\left(D^{\prime \prime}\right)=\Sigma(D)^{\prime}$, such that $\pi^{D^{\prime \prime}}$ exists, then $q^{D}(\pi) \sim q^{D^{\prime}}(\pi)$. Such a $D^{\prime \prime}$ exists (as we have seen) under the hypotheses of the theorem, but also if we assume $\left|\Sigma(D)^{\prime}\right|$ to be even, which is one of the hypotheses made by Shimura. He gives a different argument to demonstrate that $q^{D}(\pi) \sim q^{D^{\prime}}(\pi)$ if $k_{j} \geq 3$ for all $j \in \Sigma(D)^{\prime}$. Our methods also provide a slight strengthening of this result, but we again prefer to prove this fact in complete generality. The proof, which makes no direct use of the theta correspondence, will appear in $[9$, II $]$.

(d) Shimura also conjectures that the actual periods of arithmetic automorphic forms on quaternion algebras over $E$ satisfy multiplicative relations, analogous to those asserted in Theorem 2.6.1 for the quadratic periods $q^{D}(\pi)$. As explained in the introduction, the methods of this paper shed no light on this question.

More generally, the truth of the Tate conjecture would imply the existence of a factorization over $E$ of motives $M(\pi) \cong \bigotimes_{j=1}^{d} M\left(\pi^{D\left(\left\{\sigma_{j}\right\}\right)}\right)$, where $M\left(\pi^{D}\right)$ is the motive attached to the automorphic representation $\pi^{D}$, which can actually be realized, by Jannsen's methods [18], as a motive for absolute Hodge cycles. Such an isomorphism, on the level of Hodge-de Rham structures, would imply more precise period relations than those proved in the present paper, in the sense that the action of $\operatorname{Gal}(\overline{\mathbb{Q}} / \mathbb{Q})$ on the ratio of the two sides of Theorem 2.6.1 could be read in terms of the Galois action on the arguments.

When $\pi$ is of the form $\pi(\omega, \mathscr{K})$, the invariants $q^{D}(\pi)$ are denoted $q^{D}(\omega, \mathscr{K})$. These have been identified by Shimura in terms of the CM periods introduced in $\S 1$. With our normalizations, Shimura's theorem is as follows:

2.6.3. Theorem (Shimura, [42]). Let $\omega$ be a motivic Hecke character of $\mathscr{K}$. Let $D$ be a quaternion algebra over $E$ which splits over $\mathscr{K}$, and let $S(D)$ denote the set of primes at which $D$ ramifies. Suppose that, for all $v \in \Sigma \cup S(D)$, $\omega_{v} \neq \omega_{v}^{l}$. Then $\pi(\omega, \mathscr{K})^{D}$ exists, and $q^{D}(\omega, \mathscr{K}) \sim_{\overline{\mathbb{Q}}} p_{\mathscr{K}}\left(\omega / \omega^{l}, \Psi\right)$, where $\Psi$ is the unique subset of $\Sigma_{\mathscr{K}}$ such that $\Psi \cap \imath \Psi=\varnothing,\left.\stackrel{\Psi}{\Psi}\right|_{E}=\Sigma(D)$, and $\omega$ is strictly compatible with $\Psi$. 
Proof. The local conditions at $S(D)$ are necessary and sufficient for $\pi(\omega, \mathscr{K})$ to be in the discrete series at all places of $S(D)$ [17]. Thus $\pi(\omega, \mathscr{K})^{D}$ exists. The existence and uniqueness of $\Psi$ is a consequence of the local conditions at $\Sigma$, which imply that $k_{j}(\omega) \geq 2$ for all $j$. Shimura expressed his formula in terms of $p_{\mathscr{H}}(\chi,|\Psi|)^{2}$, in the notation of Remark 1.7(a), where $\omega \in X(\chi)$. But, taking into account our slightly different normalizations of the Petersson norm, our formulation follows from his because

$$
p_{\mathscr{K}}(\chi,|\Psi|)^{2} \sim_{\overline{\mathbb{Q}}} p_{\mathscr{K}}(\omega, \Psi) / p_{\mathscr{K}}(\omega, \imath \Psi) \sim_{\overline{\mathbb{Q}}} p_{\mathscr{H}}\left(\omega / \omega^{l}, \Psi\right) \quad \text { (by Lemma 1.6). }
$$

(He also only considered a fixed choice of $r$ for each $\underline{k}$. But replacing $\omega$ by $\omega \cdot\left(\|\cdot\|_{\mathbf{A}}\right)^{i}$, for $i \in \mathbb{Z}$, allows us to shift $r$ within its residue class mod 2, and the character $\omega \cdot\left(\|\cdot\|_{\mathbf{A}}\right)^{i} /\left(\omega \cdot\left(\|\cdot\|_{\mathbf{A}}\right)^{i}\right)^{i}$ is independent of $i$. Since (by (2.5.4)) $q^{D}(\pi)$ is also invariant under twists, this shows that it suffices to verify the assertion of the theorem for a fixed $r \equiv k_{j}(\bmod 2)$.

We give a different proof of Theorem 2.6.3 in $\S 7.2$.

2.6.4. Corollary (Shimura, [42]). Let $\mathscr{K}$ be a quadratic CM extension of $E$, and let $\omega$ be a motivic Hecke character of $\mathscr{H}$ such that, for some place $v_{0}$ of $E$ which does not split in $\mathscr{K}, \omega_{v_{0}} \neq \omega_{v_{0}}^{l}$. Then the conclusions of Theorem 2.6.1 are true when $\pi=\pi(\omega, \mathscr{K})$.

Proof. This is an immediate consequence of Shimura's Theorem 2.6.3 and Corollary 1.5 (which was also proved up to $\overline{\mathbb{Q}}^{\times}$by Shimura).

We will actually prove the following weaker version of Theorem 2.6.1.

2.6.5. Theorem. Let $(\underline{k}, r)$ be a $d+1$ tuple with $k_{j} \geq 2, k_{j} \equiv r(\bmod 2)$ for all $j$. Let $I \subset I^{\prime} \varsubsetneqq \Sigma, J=I^{\prime}-I$. There exists a constant $C=C(\underline{k}, I, J) \in$ $\mathbb{C}^{\times}$with the following property: Let $\pi$ be an automorphic representation of $G L(2, E)$ satisfying the hypotheses of Theorem 2.6.1, with $D_{1}=D(I), D_{2}=$ $D(J), D_{3}=D(\varnothing), D_{4}=D\left(I^{\prime}\right)$. Then

$$
q^{D_{1}}(\pi) \cdot q^{D_{2}}(\pi) \sim_{\overline{\mathbb{Q}}} C q^{D_{3}}(\pi) \cdot q^{D_{4}}(\pi) \sim_{\overline{\mathbb{Q}}} C \cdot q^{D_{4}}(\pi) .
$$

Moreover, if $d,|\Sigma-I|,|\Sigma-J|$, and $|J|$ are all even, then the local hypothesis at $v_{0}$ is unnecessary.

2.6.6. Corollary. Theorem 2.6.1 follows from the first part of Theorem 2.6.5, and it is sufficient to consider the case $|J|=1$.

Proof. Note first that, by Remark 2.6.2 (c), it suffices to prove Theorem 2.6.1 when $D_{1}=D(I), D_{2}=D(J), D_{3}=D(I \cap J), D_{4}=D(I \cup J)$, in the notation of 2.6.2(b). For $\sigma_{j} \in \Sigma$, let $D(j)=D\left(\left\{\sigma_{j}\right\}\right)$. Then Theorem 2.6.1 is clearly equivalent to the relation

$$
q^{D}(\pi) \sim_{\overline{\mathbb{Q}}} \prod_{j \in \Sigma(D)} q^{D(j)}(\pi) .
$$

By induction on $|\Sigma(D)|$, it suffices to prove Theorem 2.6.1 for $I \cap J=\varnothing$, $|J|=1$. Furthermore, by Shimura's case of Theorem 2.6.1 (cf. Remark (a) 
and $\S 7.3$ below), we may assume $\Sigma(D) \neq \Sigma$. Let $I^{\prime}=I \cup J$. Assume we have a constant $C$ such that $q^{D_{1}}(\pi) \cdot q^{D_{2}}(\pi) \sim_{\bar{Q}} C \cdot q^{D_{4}}(\pi)$ for any $\pi$ satisfying the hypotheses of Theorem 2.6.5. Take $\pi=\pi(\omega, \mathscr{K})$, for some Hecke character $\omega$ of $\mathscr{K}$ as in Corollary 2.6.4, with $\underline{k}(\omega)=\underline{k}, 1-w(\omega)=r$. Then Corollary 2.6.4 implies that $C \sim_{\overline{\mathbb{Q}}} 1$, and we are done.

\section{THETA CORRESPONDENCES FOR QUATERNION ALGEBRAS: GeNERALITIES}

3.1. Let $D$ be a quaternion algebra over $E$, and let $l: D \rightarrow D$ be a main involution, respectively, so that $N_{D}(d)=\operatorname{Tr}_{D}\left(d \cdot d^{l}\right)$. Then $(x, y)_{D}=\operatorname{Tr}_{D}\left(x \cdot y^{l}\right)$ is a nondegenerate inner product on the $E$-vector space $D$, and we let $G O(D)$ denote the group of similitudes of $(\cdot, \cdot)_{D}$ :

$$
G O(D)=\left\{g \in G L_{E}(D):(g x, g y)_{D}=\nu_{O}(g)(x, y)_{D} \text { for all } x, y \in D\right\} .
$$

The homomorphism $\nu_{O}: G O(D) \rightarrow R_{E / \mathbb{Q}} \mathbb{G}_{m}$ is defined by (3.1.1). There is a map $\rho: D^{\times} \times D^{\times} \rightarrow G O(D)$, with kernel $R_{E / \mathbb{Q}} \mathbb{G}_{m}$, embedded diagonally:

$$
\rho\left(d_{1}, d_{2}\right)(x)=d_{1} x d_{2}^{-1}, \quad x \in D ; \quad \nu_{o}\left(\rho\left(d_{1}, d_{2}\right)\right)=N_{D}\left(d_{1} \cdot d_{2}^{-1}\right) .
$$

The image of $\rho$ may thus be identified with the group $G O^{0}(D)$ introduced in $\S 2.3$, and indeed is equal to the algebraic connected component of $G O(D)$. If we define $\mathbf{t} \in G O(D)$ by $\mathbf{t}(x)=x^{l}$, then $G O(D)$ is the semidirect product of $G O^{0}(D)$ with the group of order two generated by $\mathbf{t}$.

Let $\mathscr{K}$ be a totally imaginary quadratic extension of $E$ over which $D$ splits, and let $i: \mathscr{K} \rightarrow D$ be an embedding of $\mathscr{K}$ in $D$; choose $\delta=\delta_{\mathscr{K}} \in$ $N_{D^{\times}}\left(i\left(\mathscr{K}^{\times}\right)\right)-i\left(\mathscr{K}^{\times}\right)$, as in $\S 2.3$.

Let $G U_{\mathscr{K}}(D)$ denote the subgroup of $\mathscr{K}$-linear elements of $G O(D)$. There exists a unique $l$-hermitian form $(\cdot, \cdot)_{D, \mathscr{K}}$ on $D$ such that $(\cdot, \cdot)_{D}=$ $\operatorname{Tr}_{\mathscr{K} / E}(\cdot, \cdot)_{D, \mathscr{K}}$ and such that $(\kappa, \lambda)_{D, \mathscr{K}}=\kappa \bar{\lambda}$ for $\kappa, \lambda \in \mathscr{K} \subset D$. Then $G U_{\mathscr{K}}(D)$ is the group of unitary similitudes of $(\cdot, \cdot)_{D, \mathscr{K}}$. The map $\rho$ of (3.1.2), restricted to $H_{\mathscr{K}} \times D^{\times}$, evidently has image in $G L_{\mathscr{K}}(D) \cap G O(D)$, and it is easy to see that $\rho$ induces an isomorphism $H_{\mathscr{K}} \times D^{\times} / R_{E / \mathbb{Q}} \mathbb{G}_{m} \stackrel{\sim}{\rightarrow} G U_{\mathscr{K}}(D)$; thus $G U_{\mathscr{K}}(D)$ is the group defined in 2.3. The restriction of $\nu_{O}$ to $G U_{\mathscr{K}}(D)$ is denoted $\nu_{\mathscr{K}}$. When $D$ is the matrix algebra $M(2, E)$, we write $G U_{\mathscr{K}}(s)$ for $G U_{\mathscr{K}}(D)$. Let $я \in \mathscr{K}$ be a nonzero element such that $\operatorname{Tr}_{\mathscr{K} / E}(я)=0$. Then $(\cdot, \cdot)_{D, я}:=я \cdot(\cdot, \cdot)_{D, \mathscr{K}}$ is a skew-hermitian form on $D$, whose similitude group is again given by $G U_{\mathscr{K}}(D)$.

3.2. The following construction is taken from [12, 5.1] and [13, §3]; it has been used elsewhere. Let $\mathscr{A} / E$ be an algebra with involution $\sigma$. If $V$ is an $\mathscr{A}$-vector space endowed with a hermitian or skew-hermitian bilinear form $\beta$, relative to $\sigma$, let $G \mathscr{U}(V)$ denote the group of similitudes of $(V, \beta)$, let $\nu_{\beta}: G \mathscr{U}(V, \beta) \rightarrow \mathbb{G}_{m}$ denote the similitude character, and let $\mathscr{U}(V, \beta)=$ $\operatorname{Ker}\left(\nu_{\beta}\right)$. Let $(V, \beta)$ and $\left(V^{\prime}, \beta^{\prime}\right)$ be two such spaces; we assume $\beta$ is 
skew-hermitian and $\beta^{\prime}$ is hermitian. We let (3.2.1)

$R\left(V, V^{\prime}\right)=R_{\mathscr{A}}\left(V, V^{\prime}\right)=\left\{\left(g, g^{\prime}\right) \in G \mathscr{U}(V, \beta) \times G \mathscr{U}\left(V^{\prime}, \beta^{\prime}\right) \mid \nu_{\beta}(g)=\nu_{\beta^{\prime}}\left(g^{\prime}\right)\right\}$.

Let $\operatorname{pr}_{1}: R\left(V, V^{\prime}\right) \rightarrow G \mathscr{U}(V, \beta), \operatorname{pr}_{2}:\left(V, V^{\prime}\right) \rightarrow G \mathscr{U}\left(V^{\prime}, \beta^{\prime}\right)$ be the natural projections. Then $R\left(V, V^{\prime}\right)$ is an algebraic group, and over any completion $E_{v}$ of $v$, the image $\operatorname{pr}_{i}\left(R\left(V, V^{\prime}\right)\left(E_{v}\right)\right)$ is of finite index in the corresponding similitude group. We always have the inclusion

$$
\mathscr{U}(V, \beta) \times \mathscr{U}\left(V^{\prime}, \beta^{\prime}\right) \subset R\left(V, V^{\prime}\right) .
$$

We regard $V$ as a right $\mathscr{A}$-module and $V^{\prime}$ as a left $\mathscr{A}$-module. The tensor product $V \otimes_{\mathscr{A}} V^{\prime}$, viewed as an $E$-vector space, has a natural skew-symmetric form defined by $\beta \otimes \beta^{\prime}$. The map

$i: G \mathscr{U}(V, \beta) \times G \mathscr{U}\left(V^{\prime}, \beta^{\prime}\right) \rightarrow G L\left(V \otimes_{\mathscr{A}} V^{\prime}\right) ; \quad i\left(g, g^{\prime}\right)\left(v \otimes v^{\prime}\right)=v g \otimes\left(g^{\prime}\right)^{-1} v^{\prime}$

takes $R\left(V, V^{\prime}\right)$ to $\operatorname{Sp}\left(V \otimes_{\mathscr{A}} V^{\prime}\right)$; this map is a homomorphism under the convention that similitude groups of skew-hermitian forms act on the right.

We write $G L(2)_{E}$ for $R_{E / \mathbb{Q}} G L(2)_{E}$. Let $R(D)=\left\{\left(g, g^{\prime}\right) \in G L(2) \times\right.$ $\left.G O(D) \mid \operatorname{det}(g)=\nu_{O}\left(g^{\prime}\right)\right\}$. In the notation of (3.2.1), this is $R\left(E^{2}, D\right)$, where $E^{2}$ is given the standard symplectic structure and $D$ is viewed as a quadratic space; define $\mathrm{pr}_{1}: R(D) \rightarrow G L(2), \mathrm{pr}_{2}: R(D) \rightarrow G O(D)$ as above. For any place $v$ of $E$, we write $R\left(D_{v}\right)=R(D)\left(E_{v}\right)$, etc. As remarked in [13, $\S 3$, $\mathrm{pr}_{2}$ maps $R\left(D_{v}\right)$ onto $G O\left(D_{v}\right)$ for all $v$; $\operatorname{pr}_{1}$ maps $R\left(D_{v}\right)$ onto $G L\left(2, E_{v}\right)$ for all finite $v$, and for all $v \in \Sigma(D)$; for $v \in \Sigma-\Sigma(D)$, the image of $\mathrm{pr}_{1}$ is the subgroup $G L(2, \mathbb{R})^{+} \subset G L\left(2, E_{v}\right)$ of elements with positive determinant.

Endow $E^{2}$ with the standard symplectic form $\langle\rangle=,\left(\begin{array}{cc}0 & 1 \\ -1 & 0\end{array}\right)$ and view $G L(2, E)$ as the group $G \mathrm{Sp}(W)$ of symplectic similitudes. The tensor product $\langle\cdot, \cdot\rangle \otimes(\cdot, \cdot)_{D}$ defines a nondegenerate alternating form on $\mathbf{W}=W \otimes D$. The map (3.2.2) defines a natural homomorphism

$$
j: R(D) \rightarrow \mathrm{Sp}(\mathbf{W}) .
$$

3.2.3. Fix a nontrivial additive character $\psi$ of $E \backslash E_{\mathrm{A}}$. Let $r_{\psi}$ denote the usual action of $S L(2, E)$ (Schrödinger model) on the Schwartz-Bruhat space $\mathscr{S}(D(\mathbf{A}))$ of $D(\mathbf{A})$. This is given by the following familiar formulas:

$$
\begin{gathered}
r_{\psi}\left(\left(\begin{array}{cc}
a & 0 \\
0 & a^{-1}
\end{array}\right)\right) \varphi(x)=\|a\|^{2} \cdot \varphi(x a), \\
r_{\psi}\left(\left(\begin{array}{ll}
1 & b \\
0 & 1
\end{array}\right)\right) \varphi(x)=\psi\left(b \cdot N_{D}(x)\right) \cdot \varphi(x), \\
r_{\psi}\left(\left(\begin{array}{cc}
0 & 1 \\
-1 & 0
\end{array}\right)\right) \varphi(x)=-\hat{\varphi}\left(x^{l}\right),
\end{gathered}
$$

where $\hat{\varphi}$ is the Fourier transform of $\varphi$ with respect to $\psi$ and a self-dual measure for $\psi$. For future reference, we note that, if $\mathscr{K}$ is a quadratic extension 
of $E$ which embeds in $D$, then $r_{\psi}$ extends to the quasisplit $2 \times 2$ unitary group $U_{\mathscr{K}}(s)$ by letting

$$
r_{\psi}\left(\left(\begin{array}{cc}
a & 0 \\
0 & \left(a^{l}\right)^{-1}
\end{array}\right)\right) \varphi(x)=\left\|N_{\mathscr{K} / E}(a)\right\|_{\mathbf{A}} \cdot \varphi(a \cdot x), \quad a \in \mathscr{K}_{\mathbf{A}}^{\times} .
$$

For $h \in G O(D(\mathbf{A}))$ and $\varphi \in \mathscr{S}(D(\mathbf{A}))$, let

$$
L(h) \varphi(x)=\left|\nu_{O}(h)\right|^{-1} \varphi\left(h^{-1} x\right), \quad x \in D(\mathbf{A}) .
$$

Since $\operatorname{det}(h)^{2}=\nu_{O}(h)^{4}$, these operators are unitary. The restriction of (3.2.4) to $O(D(\mathbf{A}))$ then commutes with $r_{\psi}$, and these are the usual actions associated to the dual reductive pair $(S L(2, E), O(D))$. As explained in [12, 5.1], if we let $G O(D)$ act on $S L(2, E)$ by the formula

$$
h * g=\left(\begin{array}{cc}
1 & 0 \\
0 & \nu(h)
\end{array}\right) g\left(\begin{array}{cc}
1 & 0 \\
0 & \nu(h)
\end{array}\right)^{-1},
$$

then $R(D) \cong S L(2, E) \rtimes G O(D)$ (semidirect product), and $r_{\psi}$ and $L$ together define a unitary representation, again denoted $r_{\psi}$, of $R(D(\mathbf{A}))$ on $\mathscr{S}(D(\mathbf{A}))$.

For $(g, h) \in R(D)$ and $\varphi \in \mathscr{S}(D(\mathbf{A}))$, let

$$
\theta(g, h ; \varphi)=\sum_{x \in D(E)} r_{\psi}(g, h) \varphi(x) .
$$

Let $D, D^{\prime}$ be two quaternion algebras which split over $\mathscr{K}$, and let $i: \mathscr{K} \rightarrow$ $D, i^{\prime}: \mathscr{K} \rightarrow D^{\prime}$ be embeddings as above. View $D, D^{\prime}$ as $\mathscr{K}$-vector spaces and endow them with the hermitian (resp. skew-hermitian) forms $(\cdot, \cdot)_{D, \mathscr{K}}$ and $(\cdot, \cdot)_{D^{\prime}, \mathrm{r}}$, respectively; then $(\cdot, \cdot)_{\mathbf{W}^{\prime}}:=(\cdot, \cdot)_{D, \mathscr{K}} \otimes(\cdot, \cdot)_{D^{\prime}, \text {, }}$ is a skew-hermitian form on $\mathbf{W}^{\prime}:=D \otimes_{\mathscr{K}} D^{\prime}$. Denote by $U\left(\mathbf{W}^{\prime}\right)$ the unitary group of $(\cdot, \cdot)_{\mathbf{W}^{\prime}}$, and by $G U\left(\mathbf{W}^{\prime}\right)$ the similitude group. Write $R\left(D, D^{\prime}, \mathscr{K}\right)=R\left(D, D^{\prime}\right)$, in the notation (3.2.1). The projections $\operatorname{pr}_{1}: R\left(D, D^{\prime}, \mathscr{K}\right) \rightarrow G U_{\mathscr{K}}(D), \operatorname{pr}_{2}$ : $R\left(D, D^{\prime}, \mathscr{K}\right) \rightarrow G U_{\mathscr{K}}\left(D^{\prime}\right)$ define surjective morphisms on points over $E_{v}$ for all finite $v$, and for all $v \in \Sigma$ such that $D_{v} \cong D_{v}^{\prime}$. If $v \in \Sigma$ is such that $D_{v}$ (resp. $D_{v}^{\prime}$ ) is ramified and $D_{v}^{\prime}\left(\right.$ resp. $\left.D_{v}\right)$ is split, then $\operatorname{pr}_{1}\left(\right.$ resp. $\left.\operatorname{pr}_{2}\right)$ is surjective over $E_{v}$ and the image of $\operatorname{pr}_{2}\left(\right.$ resp. $\mathrm{pr}_{1}$ ) is the identity component of $G U_{\mathscr{K}}\left(D_{v}^{\prime}\right)$ (resp. $G U_{\mathscr{K}}\left(D_{v}\right)$ ).

We may also view $D^{\prime}$ as a right $G U_{\mathscr{K}}\left(D^{\prime}\right)$-module by letting

$$
x(h, d)=h^{-1} x d, \quad h \in H_{\mathscr{K}}, d \in D^{\prime, \times}, x \in D^{\prime} .
$$

When $D^{\prime}=M(2)$, the restriction of this right action to $D^{\prime}{ }^{\times}=G L(2)$ coincides with the one used in (3.2.2).

Thus, we view $D^{\prime}$ (resp. $D$ ) as a right (resp. left) module over $G U_{\mathscr{K}}\left(D^{\prime}\right)$ (resp. $\left.G U_{\mathscr{K}}(D)\right)$, and $\mathbf{W}^{\prime}$ as a right module over $G U\left(\mathbf{W}^{\prime}\right)$. Let $\mathbf{W}^{\prime \prime}=$ $R_{\mathscr{K} / E} \mathbf{W}^{\prime}$; then $\operatorname{Tr}_{\mathscr{K} / E}(\cdot, \cdot)_{\mathbf{W}^{\prime}}$ is a skew-symmetric form, so $U\left(\mathbf{W}^{\prime}\right)$ embeds naturally in $\operatorname{Sp}\left(\mathbf{W}^{\prime \prime}\right)$. The map (3.2.2) then defines a natural homomorphism

$$
j: R\left(D, D^{\prime}, \mathscr{K}\right) \rightarrow U\left(\mathbf{W}^{\prime}\right) \rightarrow \mathrm{Sp}\left(\mathbf{W}^{\prime \prime}\right) .
$$


For any symplectic vector space $\mathscr{W}$ over $E$, let $\mathrm{Mp}(\mathscr{W})$ denote the metaplectic cover of $\operatorname{Sp}(\mathscr{W})_{\mathbf{A}}$, which we view as an extension of $\mathrm{Sp}(\mathscr{W})_{\mathbf{A}}$ by $\mathbb{C}^{\times}$ as in [28]. If $v$ is a place of $E$, we let $\operatorname{Mp}\left(\mathscr{W}_{v}\right)$ be the local metaplectic cover of $\operatorname{Sp}\left(\mathscr{W}_{v}\right)$. If $U \subset \operatorname{Sp}(\mathscr{W})_{\mathbf{A}}, \widetilde{U}$ denotes the inverse image of $U$ in $\operatorname{Mp}(\mathscr{W})$.

3.3. Lemma. Let $U=j\left(R\left(D, D^{\prime}, \mathscr{K}\right)\right) \subset \mathrm{Sp}\left(\mathbf{W}^{\prime \prime}\right)$. There exists a splitting $U \rightarrow \widetilde{U} \subset \mathrm{Mp}\left(\mathbf{W}^{\prime \prime}\right)$ whose restriction to $U \cap \mathrm{Sp}\left(\mathbf{W}^{\prime \prime}\right)(E)=j\left(R\left(D, D^{\prime}, \mathscr{K}\right)(E)\right)$ coincides with the canonical splitting defined by Weil.

Proof. Let $\mathscr{W}^{\prime \prime}$ denote the double space $\mathbf{W}^{\prime \prime} \oplus\left(-\mathbf{W}^{\prime \prime}\right)$, where $\left(-\mathbf{W}^{\prime \prime}\right)$ is $\mathbf{W}^{\prime \prime}$ with its symplectic form multiplied by -1 . Similarly, let $\mathscr{W}^{\prime}$ be the skewhermitian double space $\mathbf{W}^{\prime} \oplus\left(-\mathbf{W}^{\prime}\right)$, so that $U\left(\mathscr{W}^{\prime}\right) \subset \operatorname{Sp}\left(\mathscr{W}^{\prime \prime}\right)$. We may identify $\mathscr{W}^{\prime} \cong D \otimes_{\mathscr{K}} D^{\prime} \oplus D \otimes_{\mathscr{K}}\left(-D^{\prime}\right) \cong D \otimes_{\mathscr{K}}\left(D^{\prime} \oplus\left(-D^{\prime}\right)\right)$, and define $R\left(D,\left(D^{\prime} \oplus\left(-D^{\prime}\right)\right)\right)$ by $(3.2 .1)$, and $j^{\prime}: R\left(D,\left(D^{\prime} \oplus\left(-D^{\prime}\right)\right)\right) \rightarrow U\left(\mathscr{W}^{\prime}\right)$ by (3.2.2). As in the orthogonal case, there is an isomorphism

$$
R\left(D,\left(D^{\prime} \oplus\left(-D^{\prime}\right)\right)\right) \stackrel{\sim}{\rightarrow} U\left(D^{\prime} \oplus\left(-D^{\prime}\right)\right) \rtimes G U_{\mathscr{H}}(D) .
$$

We define an action of the right-hand side of (3.3.1) on the Schwartz-Bruhat space $\mathscr{S}\left(D(\mathbf{A})^{2}\right)$ as follows: For $h \in G U_{\mathscr{K}}(D(\mathbf{A}))$ and $\varphi \in \mathscr{S}\left(D(\mathbf{A})^{2}\right)$, let

$$
L(h) \varphi\left(\left(x_{1}, x_{2}\right)\right)=\left|\nu_{O}(h)\right|^{-2} \varphi\left(h^{-1} x_{1}, h^{-1} x_{2}\right), \quad x_{1}, x_{2} \in D(\mathbf{A}) .
$$

Now let $D^{\prime}(\Delta)=\left\{(d, d) \in D^{\prime} \oplus\left(-D^{\prime}\right)\right\}, D^{\prime}(-\Delta)=\left\{(d,-d) \in D^{\prime} \oplus\left(-D^{\prime}\right)\right\}$; then

$$
D^{\prime} \oplus\left(-D^{\prime}\right)=D^{\prime}(\Delta) \oplus D^{\prime}(-\Delta)
$$

is a full polarization of $D^{\prime} \oplus\left(-D^{\prime}\right)$. Since $D$ is even-dimensional, the formulas for the Schrödinger model, derived in this case by Kudla [ $51, \S 5]$ and reproduced in part in $\$ 3.4$ below, define a Weil representation $r_{\psi}$ of $U\left(D^{\prime} \oplus\left(-D^{\prime}\right)\right)$ on $\mathscr{S}\left(D(\mathbf{A})^{2}\right)$. As in the previous case, these actions together define an action $r_{\psi}$ of $R\left(D,\left(D^{\prime} \oplus\left(-D^{\prime}\right)\right)\right)$ on $\mathscr{S}\left(D(\mathbf{A})^{2}\right)$, which splits $\mathrm{Mp}\left(\mathscr{W}^{\prime \prime}\right)$ over the image of $R\left(D,\left(D \oplus\left(-D^{\prime}\right)\right)\right)$. But now

$$
U=j^{\prime}\left(R\left(D,\left(D^{\prime} \oplus\left(-D^{\prime}\right)\right), \mathscr{K}\right)\right) \cap\left(U\left(\mathbf{W}^{\prime}\right) \times\{1\}\right) .
$$

The restriction of $r_{\psi}$ to $U$ is the desired splitting.

3.4. Remark on splittings. The existence of splittings at all finite primes of the restriction of $\operatorname{Mp}_{v}\left(\mathbf{W}_{v}^{\prime \prime}\right)$ over $U\left(\mathbf{W}_{v}^{\prime}\right)$ is demonstrated in [28, p. $51 \mathrm{ff}$.]. This is the main reason we prefer to define the metaplectic cover as an extension by $\mathbb{C}^{\times}$, rather than the more familiar two-fold cover. An easy cocycle computation shows that $\operatorname{Mp}_{v}\left(\mathbf{W}_{v}^{\prime \prime}\right)$ splits over $U\left(\mathbf{W}_{v}^{\prime}\right)$ for archimedean primes as wellcertainly the corresponding statement is not true of the double cover for real primes. The set of splittings is a principal homogeneous space under the set of characters $U\left(\mathbf{W}_{v}^{\prime}\right) \rightarrow \mathbb{C}^{\times}=\operatorname{Ker}\left(\operatorname{Mp}\left(\mathbf{W}_{v}^{\prime \prime}\right) \rightarrow \operatorname{Sp}\left(\mathbf{W}_{v}^{\prime \prime}\right)\right)$, and Lemma 3.3 serves to normalize the splitting globally.

When the residue characteristic of $v$ is different from 2 and $v$ is inert in $\mathscr{K}$, this splitting has a different description. In that case, the covering 
$\operatorname{Mp}\left(\mathbf{W}_{v}^{\prime \prime}\right) \rightarrow \operatorname{Sp}\left(\mathbf{W}_{v}^{\prime \prime}\right)$ splits over the maximal compact subgroup $K^{\prime \prime}$ stabilizing a self-dual lattice $A^{\prime \prime} \subset \mathbf{W}_{v}^{\prime \prime}$ (the lattice model, cf. [28, II.8]), and this splitting is necessarily unique.

In what follows, we will have to consider splittings over subgroups of $j\left(R\left(D, D^{\prime}, \mathscr{K}\right)\right)$, and we will always assume them to have been induced by the doubling construction of the preceding lemma; we call this procedure "doubling $D$ (as a hermitian space)". When $D^{\prime}$ is itself split (as in $\S 3.4$, below), we use the splitting of $j\left(R\left(D, D^{\prime}, \mathscr{K}\right)\right)$ defined by a complete polarization of $D^{\prime}$; but this is equivalent to the splitting constructed in $\S 3.3$, since any two Schrödinger models for $U\left(D^{\prime} \oplus\left(-D^{\prime}\right)\right)$ define equivalent representations.

Unfortunately, some of the seesaw pairs described below introduce new dual pairs in which one of the hermitian spaces is odd dimensional, in which case the splitting cannot be defined canonically as above. The necessary modifications are described in $\S 3.6$ below.

We will need some of the formulas for the Schrödinger model below. Let $x=\left(x_{1}, x_{2}\right) \in D(\mathbf{A})^{2}$. As special cases of [51, loc.cit.], we have

$$
r_{\psi}\left(\left(\begin{array}{cc}
A & 0 \\
0 & \left(A^{l}\right)^{-1}
\end{array}\right)\right) \varphi(x)=\left\|N_{\mathscr{K} / E} \operatorname{det}(a)\right\|_{\mathbf{A}} \cdot \varphi(x \cdot A) ; A \in G L\left(2, \mathscr{K}_{\mathbf{A}}\right),
$$

$$
r_{\psi}\left(\left(\begin{array}{cc}
1 & B \\
0 & 1
\end{array}\right)\right) \varphi(x)=\psi(B[x]) \cdot \varphi(x), \quad B \in M\left(2, \mathscr{K}_{\mathbf{A}}\right) \text { hermitian } .
$$

In (3.4.2), $B[x] \in E_{\mathrm{A}}$ is the trace of the $2 \times 2$ matrix of inner products $\left.\left(\left(x_{1}, x_{2}\right),\left(x_{1}, x_{2}\right) \cdot B\right)\right)_{D, \mathscr{K}}$.

3.5. Seesaw pairs (a catalogue). Most relations between periods of automorphic forms on different groups are derived from seesaw dual reductive pairs. An abstract definition can be found in [21]; we will be content to explain a few examples, including most of those listed in [loc.cit.]. In what follows, $\mathscr{K} / E$ is a quadratic extension of local or global fields.

3.5.1. Let $(V, \beta)$ be a hermitian space, $\left(V^{\prime}, \beta^{\prime}\right)$ a skew-hermitian space, relative to the quadratic extension $\mathscr{K} / E$. Then $\left(U_{\mathscr{K}}(V), U_{\mathscr{K}}\left(V^{\prime}\right)\right)$ form a dual reductive pair, and the product $U_{\mathscr{K}}(V) \times U_{\mathscr{K}}\left(V^{\prime}\right)$ lies inside a group $R\left(V, V^{\prime}\right)$ as in (3.2.1), which acts on $V \otimes_{\mathscr{K}} V^{\prime}$. Then $\left(R_{\mathscr{K} / E}(V), \operatorname{Tr}_{\mathscr{K} / E}(\beta)\right)$ (resp. $\left.\left(R_{\mathscr{K} / E}\left(V^{\prime}\right), \operatorname{Tr}_{\mathscr{K} / E}\left(\beta^{\prime}\right)\right)\right)$ is naturally an orthogonal (resp. symplectic) space. Assume $V=\mathscr{K} \otimes W, V^{\prime}=\mathscr{K} \otimes W^{\prime}$ in such a way that $\left.\beta\right|_{W}$ is symmetric and $\left.\beta^{\prime}\right|_{W^{\prime}}$ is skew-symmetric. Then $\left(O(W), \operatorname{Sp}\left(R_{\mathscr{K} / E}\left(V^{\prime}\right)\right)\right.$ and $\left(O\left(R_{\mathscr{K} / E}(V)\right), \mathrm{Sp}\left(W^{\prime}\right)\right)$ are dual reductive pairs. The relations between these pairs and the pair $\left(U_{\mathscr{K}}(V), U_{\mathscr{K}}\left(V^{\prime}\right)\right)$ are represented by the following "seesaw 
diagrams," which we write in terms of the similitude groups:

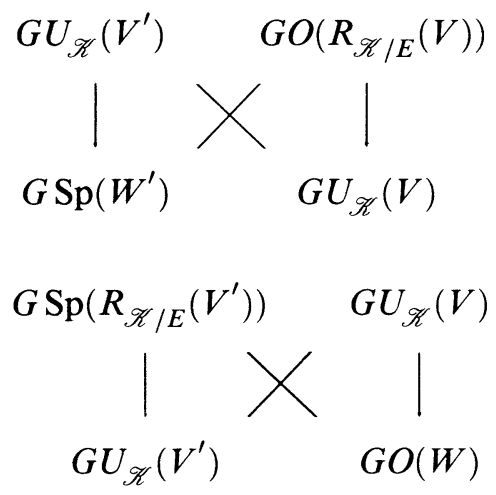

The vertical segments represent inclusions (the group below is included in the group above), the diagonal segments represent dual reductive pairs.

An example of the first diagram is given by $V=M(2, E), V^{\prime}=D$, as in $\S 3.2$, above. The $E$-structure $W$ is the space of fixed points of the main involution $l$, defined relative to the embedding $\mathscr{K} \rightarrow M(2, E)$. We write $G U_{\mathscr{H}}(s)=G U_{\mathscr{H}}(M(2, E))$; the corresponding seesaw diagram is

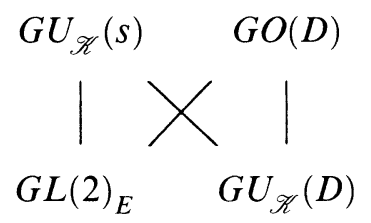

with notation as above.

3.5.2. Retain the notation of 3.5.1, and suppose $V=V_{1} \oplus V_{2}$ is an orthogonal direct sum of hermitian spaces. We then have the seesaw diagram:

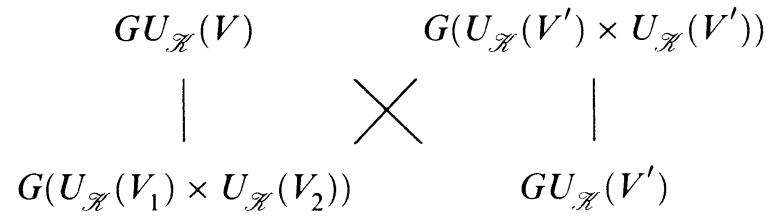

where, in general, we let

$$
\left.G\left(U_{\mathscr{H}}(V) \times U_{\mathscr{K}}(W)\right)=\left\{(g, h) \in G U_{\mathscr{H}}(V) \times G U_{\mathscr{K}}(W)\right) \mid \nu_{V}(g)=\nu_{W}(h)\right\} .
$$

When $V^{\prime}=D^{\prime}, V=D$ as above, let $V_{1}=i(\mathscr{K}), V_{2}=i(\mathscr{K}) \cdot \delta$ as above. Then (3.5.2.1) becomes

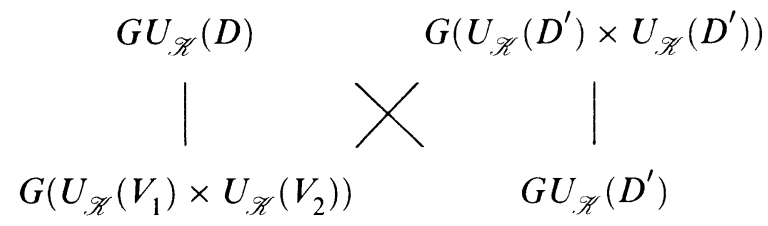


Similarly, if $D^{\prime}=M(2, E)$, we obtain a seesaw diagram:

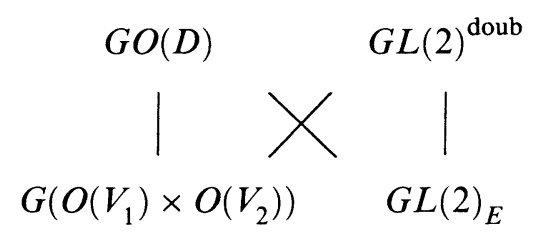

where $G L(2)^{\text {doub }}=\left\{(g, h) \in G L(2, E)^{2} \mid \operatorname{det}(g)=\operatorname{det}(h)\right\}$.

3.5.3. The doubling method [14, 34] provides another source of seesaw pairs. If $V$ and $V^{\prime}$ are as above, let $\left(-V^{\prime}\right)$ denote the skew-hermitian space $\left(V^{\prime},-\beta^{\prime}\right)$; the same definition is used for hermitian spaces in $\S 6$. We have the diagram (a special case of (3.5.2.1)):

$$
G U_{\mathscr{K}}\left(V^{\prime} \oplus\left(-V^{\prime}\right)\right)
$$

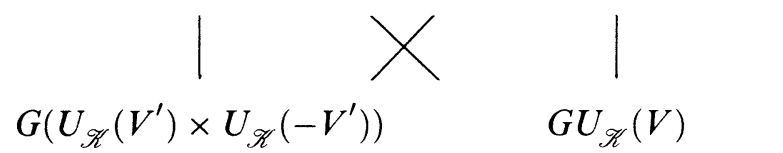

where $G\left(U_{\mathscr{H}}\left(V^{\prime}\right) \times U_{\mathscr{H}}\left(-V^{\prime}\right)\right)$ acts diagonally on $V^{\prime} \oplus\left(-V^{\prime}\right)$ and the right-hand vertical segment is the diagonal embedding.

This can be combined with (3.5.2.1) as follows:

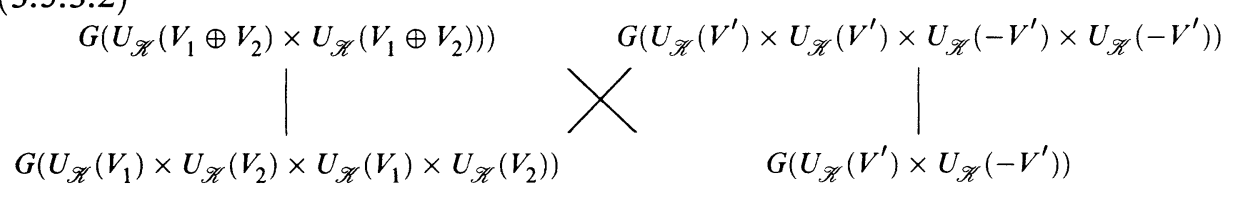

3.6. Assume that, in diagram (3.5.3.1), the space $V$ is odd dimensional. As remarked at the end of $\S 3.4$, the metaplectic covers of the groups $R\left(V, \pm V^{\prime}\right)$, can no longer be split canonically by means of the construction of Lemma 3.3. The same problem evidently arises in (3.5.3.2) if $V_{1}$ and $V_{2}$ are both odd dimensional, even though $R\left(V, V^{\prime}\right)$ has a canonical splitting in that case. Suppose $V_{1}=\mathscr{K}, V_{2}=\mathscr{K} \delta$ as in (3.5.2.2), but $V^{\prime}$ is arbitrary, of dimension $n$; we explain how to interpret the diagram (3.5.3.2) in this case, which will be sufficient for our purposes. Write $G U(\mathscr{K})$ instead of $G U_{\mathscr{K}}(\mathscr{K})$.

Note first that, as a skew-hermitian space, (3.6.1)

$\left(V_{i} \otimes_{\mathscr{K}} V^{\prime}\right) \oplus\left(V_{i} \otimes_{\mathscr{K}}\left(-V^{\prime}\right)\right) \cong\left(V_{i} \otimes_{\mathscr{K}} V^{\prime}\right) \oplus\left(\left(-V_{i}\right) \otimes_{\mathscr{K}} V^{\prime}\right) \cong\left(V_{i} \oplus\left(-V_{i}\right)\right) \otimes_{\mathscr{K}} V^{\prime}$, for $i=1,2$. Write $U_{\mathscr{K}}(n, n)$ for the quasi-split unitary group in its standard representation on $\mathscr{K}^{2 n}$, so that $U_{\mathscr{H}}\left(V_{i} \oplus\left(-V_{i}\right)\right) \cong U_{\mathscr{H}}(1,1)$. Let $\mathscr{G}_{0}$ (resp. $\mathscr{G}_{0}^{\prime}$ ) be the lower left-hand (resp. upper right-hand) corner of (3.5.3.2); then the theta-lifting from $\mathscr{G}_{0}$ to $\mathscr{G}_{0}^{\prime}$ is the same as that from

$$
\mathscr{G}:=G\left(U_{\mathscr{H}}\left(V_{1}\right) \times U_{\mathscr{H}}\left(V_{2}\right) \times U_{\mathscr{H}}\left(-V_{1}\right) \times U_{\mathscr{H}}\left(-V_{2}\right)\right)
$$

to

$$
\mathscr{G}^{\prime}:=G\left(U_{\mathscr{K}}\left(V^{\prime}\right) \times U_{\mathscr{K}}\left(V^{\prime}\right) \times U_{\mathscr{H}}\left(V^{\prime}\right) \times U_{\mathscr{H}}\left(V^{\prime}\right)\right) .
$$


Define $R\left(\mathscr{G}^{\prime}, \mathscr{G}\right) \subset \mathscr{G}^{\prime}(\mathbf{A}) \times \mathscr{G}(\mathbf{A})$ by analogy with (3.2.1). Now we may place these two in the doubled diagram for (3.5.3.1):

$$
G\left(U_{\mathscr{K}}\left(V_{1} \oplus\left(-V_{1}\right)\right) \times U_{\mathscr{K}}\left(V_{2} \oplus\left(-V_{2}\right)\right)\right) \quad \begin{aligned}
& \mathscr{G}^{\prime} \\
& G\left(U_{\mathscr{K}}\left(V^{\prime}\right) \times U_{\mathscr{K}}\left(V^{\prime}\right)\right)
\end{aligned}
$$

Now the splitting for the upper-left/lower-right diagonal can be defined by doubling the Schrödinger model for the action of $U_{\mathscr{K}}(1,1) \rtimes G U_{\mathscr{K}}\left(V^{\prime}\right)$ on $\mathscr{S}\left(V^{\prime}(\mathbf{A})\right)$. (When $V^{\prime}=D^{\prime}$, the case of interest to us, this is given by formulas (3.2.3.2-4) and (3.2.4) with $D$ replaced by $D^{\prime}$, viewing $G U_{\mathscr{K}}\left(D^{\prime}\right)$ as a subgroup of $G O\left(D^{\prime}\right)$.) This defines by restriction an action of $\mathscr{G}(\mathbf{A})$ on $\mathscr{S}\left(V^{\prime}(\mathbf{A})\right)$, which has to be extended to an action of $R\left(\mathscr{G}^{\prime}, \mathscr{G}\right)$.

It suffices to define the analogous extension for the pair $R\left(V^{\prime}, V_{i}\right)$ of (3.2.1), $i=1,2$, so that $R\left(\mathscr{G}^{\prime}, \mathscr{G}\right) \subset R\left(V^{\prime}, V_{1}\right)^{2} \times R\left(V^{\prime}, V_{2}\right)^{2}$. Now as skew-hermitian space, $V^{\prime} \oplus\left(-V^{\prime}\right) \cong \mathscr{K} \otimes_{E} W^{\prime}$, where $W^{\prime}$ is the standard symplectic space of dimension $2 n$. Thus we have a special case of diagram (3.5.1.1):

$$
G U_{\mathscr{K}}\left(V^{\prime} \oplus\left(-V^{\prime}\right)\right)
$$

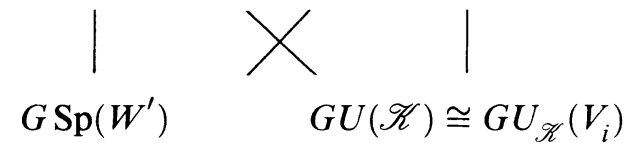

The group $R\left(W^{\prime}, R_{\left.\mathscr{K} / E^{\mathscr{K}}\right)}\right.$ of (3.2.1) is isomorphic to $\mathrm{Sp}\left(W^{\prime}\right) \rtimes$ $G O_{\mathscr{K}}\left(R_{\mathscr{K} / E^{\mathscr{K}}}\right)$ as before, and its action $r_{\psi}$ on the Schwartz-Bruhat space $\mathscr{S}=\mathscr{S}\left(\mathscr{K}(\mathbf{A})^{n}\right)$ is defined in the Schrödinger model by the analogues of the formulas (3.4.1-2), (3.3.2). Especially, if

$$
\alpha(A)=\left(\begin{array}{cc}
A & 0 \\
0 & { }^{t} A^{-1}
\end{array}\right) \in \mathrm{Sp}\left(n, E_{\mathbf{A}}\right) \cong \mathrm{Sp}\left(W^{\prime}(\mathbf{A})\right), \quad A \in G L\left(n, E_{\mathbf{A}}\right),
$$

then instead of (3.4.1) we have

$$
r_{\psi}(\alpha(A)) \varphi((x))=\varepsilon_{\mathscr{K}}(\operatorname{det}(A))\|\operatorname{det}(A)\|_{\mathbf{A}} \cdot \varphi((x) \cdot A), \quad(x) \in \mathscr{K}_{\mathbf{A}}^{n}, \varphi \in \mathscr{S},
$$

where $\varepsilon_{\mathscr{K}}: E^{\times} \backslash E_{\mathrm{A}}^{\times} \rightarrow \mathbb{C}^{\times}$is the quadratic character associated to $\mathscr{K} / E$. Now each extension $\gamma: \mathscr{K}^{\times} \backslash \mathscr{K}_{\mathbf{A}}^{\times} \rightarrow \mathbb{C}^{\times}$of $\varepsilon_{\mathscr{K}}$ to a Hecke character of $\mathscr{K}$ defines an extension of $r_{\psi}$ to a Schrödinger model $r_{\psi, \gamma}: U_{\mathscr{K}}\left(V^{\prime} \oplus\left(-V^{\prime}\right)\right) \rightarrow \operatorname{Aut}(\mathscr{S})$, uniquely determined by the formula

$$
\begin{aligned}
& r_{\psi, \gamma}\left(\alpha_{\mathscr{K}}(A)\right) \varphi((x))=\gamma(\operatorname{det}(A))\|\operatorname{det}(A)\|_{\mathbf{A}} \cdot \varphi((x) \cdot A), \quad(x) \in \mathscr{K}(\mathbf{A})^{n}, \varphi \in \mathscr{S}, \\
& \text { for }
\end{aligned}
$$

$$
\begin{gathered}
A \in G L\left(n, \mathscr{K}_{\mathbf{A}}\right), \\
\alpha_{\mathscr{K}}(A)=\left(\begin{array}{cc}
A & 0 \\
0 & \left({ }^{t} A^{-1}\right)^{l}
\end{array}\right) \in U_{\mathscr{H}}(n, n)(\mathbf{A}) \cong U_{\mathscr{K}}\left(V^{\prime} \oplus\left(-V^{\prime}\right)\right)(\mathbf{A}),
\end{gathered}
$$


where $l$ is conjugation of $\mathscr{K}$ over $E$. Then $r_{\psi, \gamma}$ likewise extends to an action of the group $R\left(\left(V^{\prime} \oplus\left(-V^{\prime}\right), V_{i}\right)\right.$ on $\mathscr{S}$. From here we reason as in the proof of Lemma 3.3 to obtain a splitting over $R\left(V^{\prime}, V_{i}\right)$. (For a different approach, cf. $[7, \S 3.2]$.)

However, in our applications, as in (3.5.3.2), we always work with the double of the pair $\left(G U_{\mathscr{K}}\left(V^{\prime} \oplus\left(-V^{\prime}\right)\right), G U_{\mathscr{H}}\left(V_{i}\right)\right)$. Thus we are free to define the Schrödinger model for the pair $\left.\left(G\left(U_{\mathscr{K}}\left(V^{\prime} \oplus\left(-V^{\prime}\right)\right)^{2}\right), G\left(U_{\mathscr{H}}\left(V_{i}\right)\right) \times U_{\mathscr{K}^{\prime}}\left(-V_{i}\right)\right)\right)$ to be the restriction of $r_{\psi, \gamma} \otimes r_{\psi, \gamma^{-1}}$, and then restrict to $R\left(V^{\prime}, V_{i}\right) \times$ $R\left(V^{\prime},\left(-V_{i}\right)\right)$. It is easy to see that this choice is in fact forced upon us by the diagram (3.6.2) when the action of $R\left(V_{1} \oplus\left(-V_{1}\right), V^{\prime}\right)$ is given by the Schrödinger model.

3.7. Let

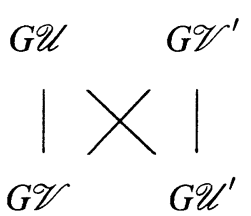

be any of the seesaw diagrams above, where the groups are similitude groups of certain bilinear forms. Let $\mathscr{U}, \mathscr{U}^{\prime}, \mathscr{V}$, and $\mathscr{V}^{\prime}$ be the corresponding symmetry groups (the kernels of the similitude homomorphisms). Define $R\left(\mathscr{U}, \mathscr{U}^{\prime}\right) \subset$ $G \mathscr{U} \times G \mathscr{U}^{\prime}, R\left(\mathscr{V}, \mathscr{V}^{\prime}\right) \subset G \mathscr{V} \times G \mathscr{V}^{\prime}$, as in (3.2.1). There are maps

$$
j_{\mathscr{U}}: R\left(\mathscr{U}, \mathscr{U}^{\prime}\right) \rightarrow \mathrm{Sp}(\mathscr{W}), \quad j_{\mathscr{V}}: R\left(\mathscr{V}, \mathscr{V}^{\prime}\right) \rightarrow \mathrm{Sp}(\mathscr{W})
$$

as in (3.2.2), for some symplectic space $\mathscr{W}$ (the same for the $\mathscr{U}$ 's and the $\mathscr{V}$ 's), such that $\left(j(\mathscr{U}), j\left(\mathscr{U}^{\prime}\right)\right)$ and $\left(j(\mathscr{V}), j\left(\mathscr{V}^{\prime}\right)\right)$ are dual reductive pairs in $\mathrm{Sp}(\mathscr{W})$ (cf. [21]). In practice, we will also be given splittings

$$
\tilde{j}_{\mathscr{U}}: R\left(\mathscr{U}, \mathscr{U}^{\prime}\right) \rightarrow \mathrm{Mp}(\mathscr{W}), \quad \tilde{\jmath}_{\mathscr{V}}: R\left(\mathscr{V}, \mathscr{V}^{\prime}\right) \rightarrow \mathrm{Mp}(\mathscr{W})
$$

which coincide on $j_{\mathscr{U}}\left(R\left(\mathscr{U}, \mathscr{U}^{\prime}\right)\right) \cap j_{\mathscr{V}}\left(R\left(\mathscr{V}, \mathscr{V}^{\prime}\right)\right)$ (see Remark 3.7.5, below). Thus, if $\psi$ is an additive character, we obtain representations, denoted generically $r_{\psi}$, of $R\left(\mathscr{U}, \mathscr{U}^{\prime}\right)$ and $R\left(\mathscr{V}, \mathscr{V}^{\prime}\right)$, on a space $\mathscr{S}$, which will usually be the space $\mathscr{S}\left(\mathscr{W}^{+}\right)$of Schwartz-Bruhat functions on a Lagrangian subspace $\mathscr{W}^{+}$of $\mathscr{W}$.

The local theta correspondence is defined as follows. First consider the nonarchimedean case. Let

$$
\begin{aligned}
& G \mathscr{U}_{+}=\operatorname{pr}_{1} R\left(\mathscr{U}, \mathscr{U}^{\prime}\right) \subset G \mathscr{U}, \quad G \mathscr{V}_{+}^{\prime}=\operatorname{pr}_{2} R\left(\mathscr{V}, \mathscr{V}^{\prime}\right) \subset G \mathscr{V}^{\prime}, \\
& G \mathscr{V}_{+}=G \mathscr{U}_{+} \cap G \mathscr{V}, \quad G \mathscr{U}_{+}^{\prime}=G \mathscr{V}_{+}^{\prime} \cap G \mathscr{U}^{\prime} \quad \text { (note the asymmetry). }
\end{aligned}
$$

Thus $\left[G \mathscr{U}: G \mathscr{U}_{+}\right]<\infty$, etc. Let $\pi$ be an admissible irreducible representation of $G \mathscr{U}_{+}^{\prime}$, and define the $G \mathscr{U}_{+}$-module

$$
\Theta^{0}\left(G \mathscr{U}^{\prime} \rightarrow G \mathscr{U} ; \pi\right)=\Theta_{\psi}^{0}\left(G \mathscr{U}^{\prime} \rightarrow G \mathscr{U} ; \pi\right)=[\mathscr{S} \otimes \pi]_{\mathscr{U}^{\prime}},
$$

the maximal quotient of $\mathscr{S} \otimes \pi$ on which $\mathscr{U}^{\prime}$ acts trivially. Here the $G \mathscr{U}_{+}$ action is deduced from the action $r_{\psi}$ of $R\left(\mathscr{U}, \mathscr{U}^{\prime}\right)$ on $\mathscr{S}$, as in [12]. It follows 
from the Howe duality conjecture, proved in residue characteristic $\neq 2$ [45], that $\Theta^{0}\left(G \mathscr{U}^{\prime} \rightarrow G \mathscr{U} ; \pi\right)$ has a unique irreducible $G \mathscr{U}_{+}$-quotient, the Howe quotient, which we denote $\Theta\left(G \mathscr{U}^{\prime} \rightarrow G \mathscr{U} ; \pi\right)=\Theta_{\psi}\left(G \mathscr{U}^{\prime} \rightarrow G \mathscr{U} ; \pi\right)$. Let

$$
\widetilde{\Theta}\left(G \mathscr{U}^{\prime} \rightarrow G \mathscr{U} ; \pi\right)=\operatorname{Ind}_{G \mathscr{U}_{+}}^{G \mathscr{U}} \Theta\left(G \mathscr{U}^{\prime} \rightarrow G \mathscr{U} ; \pi\right) .
$$

3.7.4. Lemma (local seesaw duality, cf. [21, 12]). Let $\pi$ be as above, and let $\sigma$ be an admissible irreducible representation of $G \mathscr{V}_{+}$. Then

$\operatorname{Hom}_{G \mathscr{V}_{+}}\left(\sigma \otimes \Theta^{0}\left(G \mathscr{U}^{\prime} \rightarrow G \mathscr{U} ; \pi\right), \mathbb{C}\right) \cong \operatorname{Hom}_{G \mathscr{U}_{+}^{\prime}}\left(\Theta^{0}\left(G \mathscr{V} \rightarrow G \mathscr{V}^{\prime} ; \sigma\right) \otimes \pi, \mathbb{C}\right)$

Proof. We write $\sigma$ and $\pi$ for group actions as well as spaces. Let

$$
\Theta_{\mathscr{U}^{\prime}}: \mathscr{S} \otimes \pi \rightarrow[\mathscr{S} \otimes \pi]_{\mathscr{U}^{\prime}}, \quad \Theta_{\mathscr{V}}: \sigma \otimes \mathscr{S} \rightarrow[\sigma \otimes \mathscr{S}]_{\mathscr{V}}
$$

be the natural maps. Let $\Lambda \in \operatorname{Hom}_{G \mathscr{V}}\left(\sigma \otimes \Theta\left(G \mathscr{U}^{\prime} \rightarrow G \mathscr{U} ; \pi\right), \mathbb{C}\right)$, so that, for all $v \in \sigma, \varphi \in \mathscr{S}, w \in \pi, h \in G \mathscr{V}_{+}^{+}$, we have

$$
\Lambda\left(\sigma(h) v \otimes \pi^{\prime}(h) \Theta_{\mathscr{U}^{\prime}}(\varphi \otimes w)\right)=\Lambda\left(v \otimes \Theta_{\mathscr{U}^{\prime}}(\varphi \otimes w)\right),
$$

where $\pi^{\prime}$ is the natural action of $G \mathscr{U}_{+}$on $\Theta\left(G \mathscr{U}^{\prime} \rightarrow G \mathscr{U} ; \pi\right)$. Since $h \in G \mathscr{V}_{+}$, we may find $h^{\prime} \in G \mathscr{U}^{\prime}$ such that $\nu\left(h^{\prime}\right)=\nu(h)$; necessarily $h^{\prime} \in G \mathscr{U}_{+}^{\prime}$. Then

$$
\pi^{\prime}(h) \boldsymbol{\Theta}_{\mathscr{U}^{\prime}}(\varphi \otimes w)=\Theta_{\mathscr{U}^{\prime}}\left(r_{\psi}\left(h, h^{\prime}\right) \varphi \otimes \pi\left(h^{\prime}\right) w\right)
$$

and this is independent of the choice of $h^{\prime}$, by the definition of $\Theta_{\mathscr{U}^{\prime}}$. Thus $\Lambda$ defines a map from $\sigma \otimes \mathscr{S} \otimes \pi$ to $\mathbb{C}$ with the property that

$$
\Lambda\left(\sigma(h) v \otimes r_{\psi}\left(h, h^{\prime}\right) \varphi \otimes \pi\left(h^{\prime}\right) w\right)=\Lambda(v \otimes \varphi \otimes w)
$$

whenever $h \in G \mathscr{V}_{+}, h^{\prime} \in G \mathscr{U}_{+}^{\prime}, \nu\left(h^{\prime}\right)=\nu(h)$. But this condition is symmetric in $G \mathscr{V}_{+}$and $G \mathscr{U}_{+}^{\prime}$. Note that the compatibility of the splittings $\tilde{j}_{\mathscr{U}}$ and $\tilde{j}_{\mathscr{V}}$ is used in an essential way.

3.7.5. Remark. The compatibility of the splittings $\tilde{j}_{\mathscr{U}}$ and $\tilde{j}_{\mathscr{V}}$ in general needs to be verified. If one is not careful the splittings can differ by a quadratic character attached to the quadratic space. This quadratic character is always trivial for the quadratic space defined by a quaternion algebra $D$, but may not be for the norm form on $\mathscr{K}$. But in our applications, the similitude groups $G U(\mathscr{K})$ and $G O(\mathscr{K})$ only appear in the context of the doubled diagram (3.5.3.2) with $V_{1}=V_{2}=\mathscr{K}$; hence the quadratic characters play no role.

In the archimedean case we define $G \mathscr{U}_{+}$as before; admissible $G \mathscr{U}_{+}$-modules are understood to be $\left(\operatorname{Lie}(G \mathscr{U}), K_{\infty,+}\right)$-modules, where $K_{\infty}$ is a chosen maximal compact subgroup of $G \mathscr{U}$ and $K_{\infty,+}=K_{\infty} \cap G \mathscr{U}_{+}$is not necessarily its identity component. Define $\Theta_{\psi}^{0}\left(G \mathscr{U}^{\prime} \rightarrow G \mathscr{U} ; \pi\right)$ and $\Theta_{\psi}\left(G \mathscr{U}^{\prime} \rightarrow G \mathscr{U} ; \pi\right)$ as in (3.7.2) and the discussion which follows. In the situation treated in the present paper, the most important new feature in the archimedean case is that the vanishing or not of $\Theta_{\psi}\left(G \mathscr{U}^{\prime} \rightarrow G \mathscr{U} ; \pi\right)$ may depend on $\psi$. Let $\psi^{-}(x)=\psi(-x)$, 
and define

$$
\begin{aligned}
\Theta_{ \pm}\left(G \mathscr{U}^{\prime} \rightarrow G \mathscr{U} ; \pi\right) & =\Theta_{\psi}\left(G \mathscr{U}^{\prime} \rightarrow G \mathscr{U} ; \pi\right) \oplus \Theta_{\psi^{-}}\left(G \mathscr{U}^{\prime} \rightarrow G \mathscr{U} ; \pi\right) ; \\
\widetilde{\Theta}\left(G \mathscr{U}^{\prime} \rightarrow G \mathscr{U} ; \pi\right) & =\operatorname{Ind}_{G \mathscr{U}_{+}}^{G \mathscr{U}} \Theta_{\psi}\left(G \mathscr{U}^{\prime} \rightarrow G \mathscr{U} ; \pi\right) \\
& \left(\text { if } \Theta_{\psi} \text { and } \Theta_{\psi^{-}}\right. \text {are both nontrivial) } \\
& =\operatorname{Ind}_{G \mathscr{U}_{+}}^{G \mathscr{U}} \Theta_{ \pm}\left(G \mathscr{U}^{\prime} \rightarrow G \mathscr{U} ; \pi\right) \quad \text { (otherwise). }
\end{aligned}
$$

Here $\operatorname{Ind}_{G \mathscr{U}_{+}}^{G \mathscr{U}}$ is defined to be induction from $\left(\operatorname{Lie}(G \mathscr{U}), K_{\infty,+}\right)$-modules to $\left(\operatorname{Lie}(G \mathscr{U}), K_{\infty}\right)$-modules. Then $\widetilde{\Theta}\left(G \mathscr{U}^{\prime} \rightarrow G \mathscr{U} ; \pi\right)$ is irreducible in all cases. Lemma 3.7.4 remains valid as stated.

Unfortunately, Lemma 3.7.4 concerns the full space $\Theta^{0}\left(G \mathscr{U}^{\prime} \rightarrow G \mathscr{U} ; \pi\right)$ corresponding to $\pi$, and not its Howe quotient $\Theta\left(G \mathscr{U}^{\prime} \rightarrow G \mathscr{U} ; \pi\right)$. For applications, we will need something stronger.

3.7.8. Lemma. Under the hypotheses of Lemma 3.7.4, suppose local Howe duality is valid for the pair $\left(\mathscr{U}, \mathscr{U}^{\prime}\right)$. Suppose further that either $(\mathrm{i}) \pi$ is supercuspidal or (ii) $\mathscr{U}$ is compact. Then $\Theta_{\psi}^{0}\left(G \mathscr{U}^{\prime} \rightarrow G \mathscr{U} ; \pi\right)=\Theta_{\psi}\left(G \mathscr{U}^{\prime} \rightarrow G \mathscr{U} ; \pi\right)$. This is true in particular when $\mathscr{U}^{\prime}$ is compact.

Proof. In case (ii), $\Theta_{\psi}^{0}\left(G \mathscr{U}^{\prime} \rightarrow G \mathscr{U} ; \pi\right)$ is fully reducible, so the equality is clear. In case (i), let $\overline{\mathscr{S}}$ denote the $L_{2}$-completion of $\mathscr{S}$. When $\pi$ is supercuspidal, we can use a matrix coefficient of its contragredient $\check{\pi}$ to define a projector realizing $\Theta_{\psi}^{0}\left(G \mathscr{U}^{\prime} \rightarrow G U ; \pi\right) \otimes \check{\pi}$ as an $R\left(\mathscr{U}, \mathscr{U}^{\prime}\right)$-invariant direct summand of $\overline{\mathscr{S}}$. It then follows that $\Theta_{\psi}^{0}\left(G \mathscr{U}^{\prime} \rightarrow G \mathscr{U} ; \pi\right)$ is a completely reducible $G \mathscr{U}_{+}$-module. It then follows from Howe duality that $\Theta_{\psi}^{0}\left(G \mathscr{U}^{\prime} \rightarrow G \mathscr{U} ; \pi\right)$ is irreducible.

3.8. Finally, we need to state the global version of Lemma 3.7.4. Let $F_{\mathscr{U}^{\prime}} \in$ $\mathscr{A}_{0}\left(G \mathscr{U}^{\prime}\right), F_{\mathscr{V}} \in \mathscr{A}_{0}(G \mathscr{V})$. Let $\varphi$ be an element of the adelic SchwartzBruhat space $\mathscr{S}\left(\mathscr{W}_{\mathbf{A}}^{+}\right)$on which the global representations $r_{\psi}$ are realized. Let $G \mathscr{U}(\mathbf{A})_{+}$denote the restricted direct product of the $G \mathscr{U}_{v,+}$, as $v$ runs through the places of the ground field $E$, and let $G \mathscr{U}(\mathbf{A})_{0}=G \mathscr{U}(E) \cdot G U(\mathbf{A})_{+}$. Similarly, we define $G \mathscr{U}^{\prime}(\mathbf{A})_{+}, G \mathscr{U}^{\prime}(\mathbf{A})_{0}, G \mathscr{V}(\mathbf{A})_{+}, G \mathscr{V}(\mathbf{A})_{0}$, etc. For $\left(g, g^{\prime}\right) \in$ $R\left(\mathscr{U}, \mathscr{U}^{\prime}\right)(\mathbf{A})$ let

$$
\theta_{\varphi}\left(g, g^{\prime}\right)=\sum_{x \in \mathscr{W}^{+}(E)} r_{\psi}\left(\tilde{j}_{\mathscr{U}}\left(g, g^{\prime}\right)\right)(\varphi)(x) ;
$$

for $\left(h, h^{\prime}\right) \in R\left(\mathscr{V}, \mathscr{V}^{\prime}\right)(\mathbf{A})$ we define $\theta_{\varphi}\left(h, h^{\prime}\right)$ similarly. For $g \in G \mathscr{U}(\mathbf{A})_{+}$, there exists $g^{\prime} \in G \mathscr{U}^{\prime}(\mathbf{A})_{+}$such that $\left(g, g^{\prime}\right) \in R\left(\mathscr{U}, \mathscr{U}^{\prime}\right)(\mathbf{A})$. We define

$$
\theta_{\varphi}\left(F_{\mathscr{U}^{\prime}}\right)(g)=\sum_{\mathscr{U}^{\prime}(E) \backslash \mathscr{U}^{\prime}(\mathbf{A})} \theta_{\varphi}\left(g, g^{\prime} u^{\prime}\right) F_{\mathscr{U}^{\prime}}\left(g^{\prime} u^{\prime}\right) d u^{\prime}
$$


Then $\theta_{\varphi}\left(F_{\mathscr{U}^{\prime}}\right)$ is a function on $G \mathscr{U}(E) \cap G \mathscr{U}(\mathbf{A})_{+} \backslash G \mathscr{U}(\mathbf{A})_{+}$, hence extends naturally to a function on $G \mathscr{U}(E) \backslash G \mathscr{U}(\mathbf{A})_{0}$. Likewise,

$$
\theta_{\varphi}\left(F_{\mathscr{V}}\right)\left(g^{\prime}\right)=\int_{\mathscr{V}(E) \backslash \mathscr{V}(\mathbf{A})} \theta_{\varphi}\left(g v, g^{\prime}\right) F_{\mathscr{V}}(g v) d v,
$$

extends naturally to a function on $G \mathscr{V}^{\prime}(E) \backslash G \mathscr{V}^{\prime}(\mathbf{A})_{0}$. Let $\mathscr{Z}_{\mathscr{U}^{\prime}}$ and $\mathscr{Z}_{\mathscr{V}}$ be the centers of $G \mathscr{U}^{\prime}$ and $G \mathscr{V}$, respectively, and define the scalar products

$$
\begin{gathered}
\left\langle F_{1}, F_{2}\right\rangle_{\mathscr{V}}=\int_{\mathscr{I}_{\mathscr{V}}(\mathbf{A}) G \mathscr{V}(E) \backslash G \mathscr{V}(\mathbf{A})_{0}} F_{1}\left(g_{\mathscr{V}}\right) F_{2}\left(g_{\mathscr{V}}\right) d g_{\mathscr{V}}, \\
F_{1}, F_{2} \in C^{\infty}\left(G \mathscr{V}(E) \backslash G \mathscr{V}(\mathbf{A})_{0}\right), \\
\left\langle F_{1}, F_{2}\right\rangle_{\mathscr{U}^{\prime}}=\int_{\mathscr{Z}_{\mathscr{U}^{\prime}}(\mathbf{A}) G \mathscr{U}^{\prime}(E) \backslash G \mathscr{U}^{\prime}(\mathbf{A})_{0}} F_{1}\left(g_{\mathscr{U}^{\prime}}\right) F_{2}\left(g_{\mathscr{U}^{\prime}}\right) d g_{\mathscr{U}^{\prime}}, \\
F_{1}, F_{2} \in C^{\infty}\left(G \mathscr{U}^{\prime}(E) \backslash G \mathscr{U}^{\prime}(\mathbf{A})_{0}\right)
\end{gathered}
$$

whenever the product $F_{1} \cdot F_{2}$ factors through the quotient by the center.

3.8.4. Proposition (seesaw reciprocity). The measures $d g_{\mathscr{V}}$ and $d g_{\mathscr{U}}$ can be normalized so that, for all $F_{\mathscr{U}^{\prime}}$ and $F_{\mathscr{V}}$ as above and all $\varphi \in \mathscr{S}\left(\mathscr{W}_{\mathbf{A}}^{+}\right)$,

$$
\left\langle\theta_{\varphi}\left(F_{\mathscr{U}}\right), F_{\mathscr{V}}\right\rangle_{\mathscr{V}}=\left\langle\theta_{\varphi}\left(F_{\mathscr{V}}\right), F_{\mathscr{U}^{\prime}}\right\rangle_{\mathscr{U}^{\prime}} .
$$

Proof. Let $G \mathscr{V}(E)_{+}=G \mathscr{V}(E) \cap G \mathscr{V}(\mathbf{A})_{+}, G \mathscr{U}^{\prime}(E)_{+}=G \mathscr{U}^{\prime}(E) \cap G \mathscr{U}^{\prime}(\mathbf{A})_{+}$. Then

$$
\begin{aligned}
& \left\langle\theta_{\varphi}\left(F_{\mathscr{U}^{\prime}}\right), F_{\mathscr{V}}\right\rangle_{\mathscr{V}}=\int_{\mathscr{Z}_{\mathscr{V}}(\mathbf{A}) G \mathscr{V}(E)_{+} \backslash G \mathscr{V}(\mathbf{A})_{+}} \theta_{\varphi}\left(F_{\mathscr{U ^ { \prime }}}\right)\left(g_{\mathscr{V}}\right) F_{\mathscr{V}}\left(g_{\mathscr{V}}\right) d g_{\mathscr{V}}, \\
& \left\langle\theta_{\varphi}\left(F_{\mathscr{V}}\right), F_{\mathscr{U}^{\prime}}\right\rangle_{\mathscr{U}^{\prime}}=\int_{\mathscr{Z}_{\mathscr{U}^{\prime}}(\mathbf{A}) G \mathscr{U}^{\prime}(E)_{+} \backslash G \mathscr{U}^{\prime}(\mathbf{A})_{+}} \theta_{\varphi}\left(F_{\mathscr{V}}\right)\left(g_{\mathscr{U}^{\prime}}\right) F_{\mathscr{U}^{\prime}}\left(g_{\mathscr{U}^{\prime}}\right) d g_{\mathscr{U}^{\prime}} ;
\end{aligned}
$$

here we are using the easily verified fact that $\mathscr{Z}_{\mathscr{V}}(\mathbf{A}) \subset G \mathscr{V}(\mathbf{A})_{+}, \mathscr{Z}_{\mathscr{U}^{\prime}}(\mathbf{A}) \subset$ $G \mathscr{U}^{\prime}(\mathbf{A})_{+}$. But now the first integral can be rewritten

$$
\int_{\Xi_{\mathscr{V}}} \int_{\mathscr{V}(E) \backslash \mathscr{V}(\mathbf{A})} \int_{\mathscr{U}^{\prime}(E) \backslash \mathscr{U ^ { \prime }}(\mathbf{A})} \theta_{\varphi}\left(v \tilde{g}_{\mathscr{V}}, g^{\prime} u^{\prime}\right) F_{\mathscr{U}^{\prime}}\left(g^{\prime} u^{\prime}\right) F_{\mathscr{V}}\left(v \tilde{g}_{\mathscr{V}}\right) d u^{\prime} d v d \tilde{g}_{\mathscr{V}},
$$

where $\tilde{g}_{\mathscr{V}}$ is a variable in $\Xi_{\mathscr{V}}=\mathscr{Z}_{\mathscr{V}}(\mathbf{A}) \cdot G \mathscr{V}(E)_{+} \cdot \mathscr{V}(\mathbf{A}) \backslash G \mathscr{V}(\mathbf{A})_{+}$. On the other hand, $g^{\prime}$ and $\tilde{g}_{\mathscr{V}}$ determine each other mutually, and we may identify $\Xi_{\mathscr{V}} \cong \Xi_{\mathscr{U}^{\prime}}:=\mathscr{Z}_{\mathscr{U}^{\prime}}(\mathbf{A}) \cdot G \mathscr{U}^{\prime}(E)_{+} \cdot \mathscr{U}^{\prime}(\mathbf{A}) \backslash G \mathscr{U}^{\prime}(\mathbf{A})_{+}$, by the very definition of $G \mathscr{U}^{\prime}(\mathbf{A})_{+}$and $G \mathscr{V}(\mathbf{A})_{+}$. Thus (3.8.4.1) is symmetric in $\mathscr{U}^{\prime}$ and $\mathscr{V}$; the proposition follows.

\section{LOCAL THETA CORRESPONDENCES FOR QUATERNION ALGEBRAS}

In this section, we work out the local theta correspondences for the pairs $(G L(2) \times G O(D))$ and $\left(G U_{\mathscr{H}}(D) \times G U_{\mathscr{H}}\left(D^{\prime}\right)\right)$, where we write $G L(2)=$ $G L(2, E)$. Thus we modify our notation and assume $E$ to be a local field of characteristic zero, $D$ and $D^{\prime}$ to be quaternion algebras over $E$, and $\mathscr{K}$ to 
be a quadratic extension given with embeddings $i: \mathscr{K} \rightarrow D$ and $i^{\prime}: \mathscr{K} \rightarrow D^{\prime}$. The quaternion algebras $D$ and $D^{\prime}$ are allowed to be split, and $\mathscr{K}$ may be the split quadratic extension $E \oplus E$. All representations of $G L(2)$ (and of $D^{\times}$, when the latter is split) are assumed to be infinite-dimensional.

The set of admissible irreducible representations of a reductive algebraic group $G$ over $E$ (or $\left(\mathfrak{g}, K_{\infty}\right)$-modules, when $E$ is archimedean) is denoted $\operatorname{Rep}(G)$. If $\pi \in \operatorname{Rep}(G)$, we denote by $\xi_{\pi}$ the central character $Z_{G} \rightarrow \mathbf{C}^{\times}=$ $\operatorname{Aut}_{G}(\pi)$. If $\omega: \mathscr{K}^{\times} \rightarrow \mathbb{C}^{\times}$is a character of $\mathscr{K}^{\times}$, we let $\xi_{\omega}: E^{\times} \rightarrow \mathbb{C}^{\times}$denote the restriction of $\omega$ to $E^{\times}$. The homomorphism (3.1.2) $D^{\times} \times D^{\times} \rightarrow G O(D)$, with image $G O^{0}(D)$, allows us to index the set $\operatorname{Rep}(G O(D))$ by pairs $\pi \otimes \pi^{\prime}$, where $\pi, \pi^{\prime} \in \operatorname{Rep}\left(D^{\times}\right), \xi_{\pi} \cdot \xi_{\pi^{\prime}}=1$, together with information on the action of the element $\mathbf{t} \in G O(D)$ of order two. The isomorphism $\mathscr{K}^{\times} \times D^{\times} \backslash E^{\times} \stackrel{\sim}{\rightarrow}$ $G U_{\mathscr{K}}(D)$ of $\S 3.1$ identifies $\operatorname{Rep}\left(G U_{\mathscr{K}}(D)\right)$ with the set of pairs $\omega \otimes \pi, \omega$ a character of $\mathscr{K}^{\times}, \pi \in \operatorname{Rep}\left(D^{\times}\right)$, such that $\xi_{\omega} \cdot \xi_{\pi}=1$.

Let $\varepsilon(D)=1$ if $D$ is split, $\varepsilon(D)=-1$ if $D$ is a division algebra. Let $\pi_{\mathscr{K}} \in \operatorname{Rep}(G L(2, \mathscr{K}))$ denote the representation obtained from $\pi$ by base change. For any $\tau \in \operatorname{Rep}(G L(2, \mathscr{K}))$, let $\varepsilon\left(\tau, s, \psi \circ \operatorname{Tr}_{\mathscr{K} / E}\right)$ denote the $\varepsilon$ factor in the functional equation of the standard local zeta function of $\tau$, so that $s=\frac{1}{2}$ is the center of symmetry if $\tau$ has a unitary central character. Here $s$ is a complex variable, $\psi$ is a character of $E$, and the $\varepsilon$-factor is defined relative to the Haar measure on $E$ which is self-dual relative to $\psi \circ \operatorname{Tr}_{\mathscr{K} / E}$. If $\xi_{\omega} \cdot \xi_{\pi}=1$, the value $\varepsilon\left(\pi_{\mathscr{K}} \otimes \omega, \frac{1}{2}, \psi \circ \operatorname{Tr}_{\mathscr{K} / E}\right)= \pm 1$ is independent of $\psi$ (cf. [44]), and is denoted $\varepsilon\left(\pi_{\mathscr{K}} \otimes \omega, \frac{1}{2}\right)$.

Let $D$ and $D^{\prime}$ be two quaternion algebras. If $\pi \in \operatorname{Rep}(G L(2))$ let $\pi^{D} \in$ $\operatorname{Rep}\left(D^{\times}\right), \pi^{D^{\prime}} \in \operatorname{Rep}\left(D^{\prime}, \times\right)$, denote the representations which correspond to $\pi$ under the Jacquet-Langlands correspondence; we make the convention that $\pi^{D}=\{0\}$ if $D$ is ramified and $\pi$ is not in the discrete series. The theta correspondence $\Theta: \operatorname{Rep}\left(G U_{\mathscr{K}}\left(D^{\prime}\right)\right) \rightarrow \operatorname{Rep}\left(G U_{\mathscr{K}}(D)\right)$ is defined below. The main result of the present section is a part of the following theorem, which has been proved jointly by Kudla and the author.

4.1. Theorem. Let $\omega \otimes \pi^{D^{\prime}} \in \operatorname{Rep}\left(G U_{\mathscr{K}}\left(D^{\prime}\right)\right)$. The theta lift $\Theta\left(\omega \otimes \pi^{D^{\prime}}\right) \neq 0$ if and only if $\varepsilon\left(\pi_{\mathscr{K}} \otimes \omega, \frac{1}{2}\right) \varepsilon(D) \cdot \varepsilon\left(D^{\prime}\right) \cdot \xi_{\pi}(-1)=1$. If this condition is satisfied, then $\pi^{D}$ exists and $\Theta\left(\omega \otimes \pi^{D^{\prime}}\right)=\omega^{-1} \otimes \check{\pi}^{D}$.

The proof will appear in forthcoming joint work with S. Kudla. For the reader's convenience, we include here short proofs in the two cases needed in this paper: (i) $D$ and $D^{\prime}$ arbitrary, $E=\mathbb{R}, \mathscr{K}=\mathbb{C}$; (ii) $E$ nonarchimedean and $D^{\prime}$ split.

4.2. The case of $D$ split is easily reduced to the theta correspondence for the pair $\left(G L(2) \times G O\left(D^{\prime}\right)\right)$. This has been worked out by Shimizu [35], and we recall his results briefly. We have constructed unitary representations $r_{\psi}$, of $R(D)$ on $\mathscr{S}\left(D^{\prime}\right)$, where $\mathscr{S}\left(D^{\prime}\right)$ is now the space of Schwartz-Bruhat functions on the four-dimensional $E$-vector space $D$. First suppose $E$ nonarchimedean. 
If $\pi \in \operatorname{Rep}(G L(2))$, let $\Theta(\pi)=\Theta^{0}\left(G L(2) \rightarrow G O\left(D^{\prime}\right) ; \pi\right)=\left[\mathscr{S}\left(D^{\prime}\right) \otimes \pi\right]_{S L(2)}$, in the notation of (3.7.2). In this case $\Theta(\pi)=\Theta\left(G L(2) \rightarrow G O\left(D^{\prime}\right) ; \pi\right)$ is already irreducible. Indeed, when $D^{\prime}$ is ramified or $\pi$ is supercuspidal this follows from Lemma 3.7.8. When $D^{\prime}$ is split and $\pi$ is principal series or special this can be deduced by computing the Jacquet module of $\Theta(\pi)$, as in [28, $\S 3]$; we omit the details. Furthermore, $\boldsymbol{\Theta}(\pi)$ does not depend on $\psi$ ([35], cf. [13] for the extension to similitude groups). More precisely,

If $D^{\prime}$ is split, then $D^{\prime, \times} \cong G L(2), \operatorname{Rep}\left(D^{\prime, \times}\right) \cong \operatorname{Rep}(G L(2))$, and $\Theta(\pi) \cong \pi \otimes \check{\pi}$, where $\check{\pi}$ is the contragredient of $\pi$.

If $D^{\prime}$ is a division algebra, then $\Theta(\pi)=0$ unless $\pi$ is a discrete series representation. If $\pi$ is a discrete series representation, then $\pi^{D^{\prime}}$ exists and $\Theta(\pi) \cong \pi^{D^{\prime}} \otimes \check{\pi}^{D^{\prime}}$.

We also have to specify the action of the element $\mathbf{t} \in G O\left(D^{\prime}\right)$ on $\Theta(\pi)$. As explained in [13, §7], the representation $\pi^{D^{\prime}} \otimes \check{\pi}^{D^{\prime}}$ of $G O^{0}\left(D^{\prime}\right)$ has two extensions to $G O\left(D^{\prime}\right)$; the extension given by $\Theta(\pi)$ is characterized by the conditions of Lemma 7.2.3 (equivalently, Corollary 7.2.4) of [13].

Finally, every $\sigma \in \operatorname{Rep}\left(D^{\prime, \times}\right)$ is of the form $\pi^{D^{\prime}}$, for some $\pi \in \operatorname{Rep}(G L(2))$. If $\pi^{D^{\prime}} \otimes \pi^{\prime, D^{\prime}} \in \operatorname{Rep}\left(G O\left(D^{\prime}\right)\right)$, let

$$
\Theta^{\prime}\left(\pi^{D^{\prime}} \otimes \pi^{\prime, D^{\prime}}\right)=\widetilde{\Theta}\left(G O\left(D^{\prime}\right) \rightarrow G L(2) ; \pi^{D^{\prime}} \otimes \pi^{\prime, D^{\prime}}\right)
$$

(notation 3.7.3 or 3.7.7). Since the reduced norm from $D^{\prime, x}$ to $E^{\times}$is surjective for $E$ nonarchimedean, $\Theta_{\psi} \neq \widetilde{\Theta}$ is only possible at ramified archimedean places. In this case, it can be verified easily (cf. §4.4) that $\Theta_{\psi}$ is a nontrivial irreducible discrete series $(\mathfrak{g l}(2, \mathbb{R}), S O(2))$-module, and then $\widetilde{\Theta}=\Theta_{ \pm}$is the corresponding discrete series $(\mathfrak{g l}(2, \mathbb{R}), O(2))$-module.

Similarly, by [17, Theorem 4.2] and the preceding discussion, we have:

Let $\Pi$ be an irreducible representation of $G O\left(D^{\prime}\right)$ whose restriction to $G O^{0}(D)$ contains $\pi^{D^{\prime}} \otimes \pi^{\prime} D^{\prime}$. Then $\Theta^{\prime}(\Pi)=0$ unless $\pi^{\prime}=\check{\pi}$ and $\Pi$ satisfies Lemma 7.2.3 of [13]; in that case, $\Theta^{\prime}(\Pi)=\pi$.

4.3. We next consider the pair $\left(G U_{\mathscr{K}}(s) \times G U_{\mathscr{H}}\left(D^{\prime}\right)\right)$, where $G U_{\mathscr{H}}(s)=G U_{\mathscr{H}}(D)$ for $D$ split. Then we have the seesaw pair (3.5.1.3):

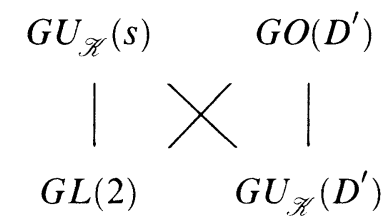

If $\omega \otimes \pi \in \operatorname{Rep}\left(G U_{\mathscr{K}}(s)\right)$ (resp. $\omega \otimes \pi^{D^{\prime}} \in \operatorname{Rep}\left(G U_{\mathscr{K}}\left(D^{\prime}\right)\right)$ ), let

$$
\begin{aligned}
\Theta(\omega \otimes \pi) & =\Theta\left(G U_{\mathscr{K}}(s) \rightarrow G U_{\mathscr{K}}\left(D^{\prime}\right) ; \omega \otimes \pi\right), \\
\Theta^{\prime}\left(\omega \otimes \pi^{D^{\prime}}\right) & =\Theta\left(G U_{\mathscr{H}}\left(D^{\prime}\right) \rightarrow G U_{\mathscr{H}}(s) ; \omega \otimes \pi^{D^{\prime}}\right),
\end{aligned}
$$


relative to the given Schrödinger model on $\mathscr{S}\left(D^{\prime}\right)$. For real places at which $D^{\prime}$ ramifies, we let $\Theta^{\prime}\left(\omega \otimes \pi^{D^{\prime}}\right)=\widetilde{\Theta}\left(G U_{\mathscr{K}}\left(D^{\prime}\right) \rightarrow G U_{\mathscr{K}}(s) ; \omega \otimes \pi\right)$, as in (3.7.7). For the purposes of the statement of Theorem 4.1, we define $\Theta\left(\omega \otimes \pi^{D}\right)$ in the same way, if $D$ is ramified and $D^{\prime}$ split; however, it is unnecessary to work out this case separately, because the nonvanishing of the theta lift is symmetric in the two groups. We note that, for all $k \in H_{\mathscr{K}}(\mathbf{A}),\left(\left(k, 1_{D^{\prime}}\right),\left(k, 1_{D}\right)\right)$, where $1_{D}$, $1_{D^{\prime}}$ are the respective identity elements, belongs to the center of $R\left(D, D^{\prime}, \mathscr{K}\right)$. Moreover, if $j: R\left(D, D^{\prime}, \mathscr{K}\right) \rightarrow U\left(\mathbf{W}^{\prime}\right)$ is defined as above, then $j(k, k)=1$. Thus if $\Theta^{\prime}\left(\omega \otimes \pi^{D^{\prime}}\right)$ is nontrivial, it must be of the form $\omega^{-1} \otimes \pi^{\prime}$ for some $\pi^{\prime} \in \operatorname{Rep}\left(D^{\times}\right)$. Write $\Theta^{\prime, 0}\left(\omega \otimes \pi^{D^{\prime}}\right)=\Theta^{\prime, 0}\left(G U_{\mathscr{H}}\left(D^{\prime}\right) \rightarrow G U_{\mathscr{H}}(s) ; \omega \otimes \pi^{D^{\prime}}\right)$.

Suppose $\Theta^{\prime}\left(\omega \otimes \pi^{D^{\prime}}\right)=\omega^{-1} \otimes \pi^{\prime} \neq\{0\}$. Then $\Theta^{\prime}\left(\omega \otimes \pi^{D^{\prime}}\right)$ restricts nontrivially to $\pi^{\prime} \in \operatorname{Rep}(G L(2))$. By seesaw duality (Lemma 3.7.4), we have (4.3.1)

$$
\begin{aligned}
\operatorname{Hom}_{G L(2)}\left(\Theta^{\prime}\left(\omega \otimes \pi^{D^{\prime}}\right) \otimes \check{\pi}^{\prime}, \mathbb{C}\right) & \subset \operatorname{Hom}_{G L(2)}\left(\Theta^{\prime, 0}\left(\omega \otimes \pi^{D^{\prime}}\right) \otimes \check{\pi}^{\prime}, \mathbb{C}\right) \\
& \cong \operatorname{Hom}_{G U_{\mathscr{H}}\left(D^{\prime}\right)}\left(\left(\omega \otimes \pi^{D^{\prime}}\right) \otimes \Theta\left(\check{\pi}^{\prime}\right), \mathbb{C}\right),
\end{aligned}
$$

(see Remark 4.3.3 for the archimedean case). It follows that there exists a nonzero $G U_{\mathscr{K}}\left(D^{\prime}\right)$-invariant pairing

$$
\left[\left.\Theta\left(\check{\pi}^{\prime}\right)\right|_{G U_{\mathscr{H}}\left(D^{\prime}\right)}\right] \otimes\left[\omega \otimes \pi^{D^{\prime}}\right] \rightarrow \mathbb{C}
$$

But $\Theta\left(\check{\pi}^{\prime}\right)=\check{\pi}^{\prime} D^{\prime} \otimes \pi^{\prime}, D^{\prime}$ by (4.2.2). It follows from (4.3.1) that

The space $\Theta^{\prime}\left(\omega \otimes \pi^{D^{\prime}}\right)=\omega^{-1} \otimes \pi^{\prime} \neq\{0\}$ if and only if $(\alpha) \pi^{\prime} \cong \check{\pi}$, and $(\beta)$ there exists a nonzero $H_{\mathscr{K}}$-invariant pairing $\omega \otimes \pi^{D^{\prime}} \rightarrow \mathbb{C}$.

4.3.3. Remark. In the archimedean case, when $D^{\prime}$ is ramified and $D$ is split, it can be verified using [19] (cf. $\S 4.4$ below) that, in the notation of $\S 3.7$, both $\Theta_{\psi}\left(\omega \otimes \pi^{D^{\prime}}\right)$ or $\Theta_{\psi^{-}}\left(\omega \otimes \pi^{D^{\prime}}\right)$ are nontrivial if either of them is. In particular, the assertion (4.3.1) follows from Lemma 3.7.4 and Frobenius reciprocity.

4.3.3. Lemma. The space $\Theta^{\prime}\left(\omega \otimes \pi^{D^{\prime}}\right) \neq\{0\}$ if and only if $\varepsilon\left(\pi_{\mathscr{K}} \otimes \omega, \frac{1}{2}\right)$. $\varepsilon\left(D^{\prime}\right) \cdot \xi_{\pi}(-1)=1$, and in that case $\Theta^{\prime}\left(\omega \otimes \pi^{D^{\prime}}\right)=\omega^{-1} \otimes \check{\pi}$.

Proof. If $\mathscr{K}$ is split, then (4.3.2) $(\beta)$ is satisfied if and only if $\varepsilon\left(D^{\prime}\right)=1$, by the theory of the Kirillov model (cf. [46, Lemma 8]). In that case, $\omega=\left(\omega_{1}, \omega_{2}\right)$ is a pair of characters of $E^{\times}$with the property that $\omega_{1} \cdot \omega_{2} \cdot \xi_{\pi}=1$. With the obvious convention: $\varepsilon\left(\pi_{\mathscr{K}} \otimes \omega, \frac{1}{2}\right)=\varepsilon\left(\pi \otimes \omega_{1}, \frac{1}{2}\right) \cdot \varepsilon\left(\pi \otimes \omega_{2}, \frac{1}{2}\right)$, note that $\omega_{1} \cdot \omega_{2} \cdot \xi_{\pi}=1$ implies that $\pi \otimes \omega_{1}$ and $\pi \otimes \omega_{2}$ are contragredient to one another. Then it is elementary (cf., e.g., $[33,8.1 .3]$ ) that

$$
\varepsilon\left(\pi \otimes \omega_{1}, \frac{1}{2}\right) \cdot \varepsilon\left(\pi \otimes \omega_{2}, \frac{1}{2}\right)=\xi_{\pi \otimes \omega_{1}}(-1)=\xi_{\pi}(-1) \cdot \omega_{1}(-1)^{2}=\xi_{\pi}(-1),
$$

so $\varepsilon\left(\pi_{\mathscr{K}} \otimes \omega, \frac{1}{2}\right) \cdot \varepsilon\left(D^{\prime}\right) \cdot \xi_{\pi}(-1)=1$, as claimed. 
If $\mathscr{K}$ is not split then the following theorem of Tunnell [44] and Waldspurger [48], recently generalized by $H$. Saito [50], translates (4.3.2) into the condition of the lemma.

4.3.4. Theorem [44, 48]. Let $\mathscr{K}$ be a nontrivial quadratic extension of a local field $E$. Let $D$ and $D^{\prime}$ denote the two nonisomorphic quaternion algebras over $E$; embed $\mathscr{K}^{\times}$as a subgroup of $D^{\times}$and $D^{\prime, \times}$. With $\omega$ and $\pi$ as above,

$$
\operatorname{dim} \operatorname{Hom}_{\mathscr{K}^{\times}}\left(\omega \otimes \pi^{D}, \mathbb{C}\right)+\operatorname{dim} \operatorname{Hom}_{\mathscr{K}^{\times}}\left(\omega \otimes \pi^{D^{\prime}}, \mathbb{C}\right)=1,
$$

and $\operatorname{Hom}_{\mathscr{K}^{\times}}\left(\omega \otimes \pi^{D}, \mathbb{C}\right) \neq 0$ if and only if $\varepsilon\left(\pi_{\mathscr{K}} \otimes \omega, \frac{1}{2}\right) \cdot \xi_{\pi}(-1)=\varepsilon(D)$.

The references cited only treat explicitly the case of nonarchimedean $E$, with $\varepsilon(D)=1$. The case $\varepsilon(D)=-1, E$ nonarchimedean, and the statement about the dimensions follows from the remarks preceding Theorem 8.2 of [33]. In the real case, the $\varepsilon$-factors are easily computed, and the assertion is an elementary consequence of the structure theory of irreducible $(\mathfrak{g l}(2, \mathbb{R}), O(2))$ modules (cf., e.g. $[17, \S 5])$ and the representation theory of $S U(2)$. Note that the result is again independent of the choice of additive character $\psi$.

4.4. It remains to treat the case $E=\mathbb{R}, D=D^{\prime}$ a division algebra. In this case, the theta correspondence between $U_{\mathscr{K}}(D)$ and $U_{\mathscr{K}}(D)$ can be extracted from [19], although the connection with epsilon factors does not seem to have been noticed previously. Before translating the results of [19] into the present language, we make the following concrete interpretation of Theorem 4.1 for $E=\mathbb{R}$. This is, in a sense, the principal observation of the present paper:

4.4.1. Corollary. Let $E=\mathbb{R}, \mathscr{K}=\mathbb{C}$, and suppose $D$ is the algebra of Hamiltonian quaternions. Let $\omega \otimes \pi^{D}$ be an irreducible, necessarily finitedimensional representation of $G U_{\mathscr{K}}(D)$. Let $k(\pi)=\operatorname{dim}\left(\omega \otimes \pi^{D}\right)+1$ be the weight, in the sense of classical holomorphic modular forms, associated to the discrete series representation $\pi$ of $G L(2, \mathbb{R})$, and define $k(\omega)$ as in $\S 1.1$. Then $\Theta\left(G U_{\mathscr{K}}(D) \rightarrow G U_{\mathscr{K}}\left(D^{\prime}\right) ; \omega \otimes \pi^{D}\right) \neq 0$ precisely in the following cases:

(i) The algebra $D^{\prime}$ is split and $k(\omega)<k(\pi)$; or

(ii) The algebra $D^{\prime}$ is ramified and $k(\omega)>k(\pi)$.

It is easy to see that this is equivalent to Theorem 4.1, using known formulas for the epsilon factors of representations of the Weil group of $\mathbb{C}$. Alternatively, when $D^{\prime}$ is split, one can apply Theorem 4.3.4 and the fact that $\omega$ occurs in $\pi$ (resp. $\pi^{D}$ ) if and only if $k(\omega)$ and $k(\pi)$ have opposite parity and $k(\omega)<k(\pi)$ (resp. $k(\omega)<k(\pi))$. Note that $k(\omega)$ and $k(\pi)$ are necessarily of different parity, since $\xi_{\omega} \cdot \xi_{\pi}=1$ (cf. the remarks following Proposition 1.8.2).

When $D=D^{\prime}=\mathbb{H}$, the corollary is a special case of [19, III, Theorem 7.2]. In their notation, we have $k=2$ and $(p, q)=(2,0)$ or $(0,2)$ depending on $\pi$. The representation $\omega \otimes \pi^{D}$, restricted to $U(k, 0)=U(2,0)$-denote this restriction $\left(\omega \otimes \pi^{D}\right)_{U}$-lifts to $U(0,2)$ (resp. to $\left.U(2,0)\right)$ if and only if the associated parameter $\lambda$ is of the form $\left(n_{1}, n_{2}\right)$ (resp. $\left(-n_{2},-n_{1}\right)$ ), with $n_{1} \geq n_{2}>0$. In the former case $\left(\omega \otimes \pi^{D}\right)_{U}$ lifts to the restriction to $U(p, q)$ of the dual representation $\omega^{-1} \otimes \check{\pi}^{D}$ and it follows immediately that, in the 
similitude correspondence, $\omega \otimes \pi^{D}$ lifts to $\omega^{-1} \otimes \check{\pi}^{D}$. Replacing the additive character $\psi$ by $\psi^{-}$has the same effect as replacing $U(p, q)$ by $U(q, p)$. Thus [19] asserts that, in the notation of (3.7.7),

$$
\widetilde{\Theta}_{\psi}\left(\omega \otimes \pi^{D}\right)=\operatorname{Ind}_{G U_{+}}^{G U} \Theta_{ \pm}\left(\omega \otimes \pi^{D}\right) \cong \omega^{-1} \otimes \check{\pi}^{D} \neq 0
$$

if and only if the parameter is the form $\left(n_{1}, n_{2}\right)$ or $\left(-n_{2},-n_{1}\right)$, with $n_{1} \geq$ $n_{2}>0$. It remains to observe that if the parameter of $\left(\omega \otimes \pi^{D}\right)_{U}$ is of the form $(a, b)$, in the usual parametrization of representations of $U(2)$, then $k(\pi)=a-b+2, k(\omega)=|a+b|+1$. The condition $k(\pi)<k(\omega)$ then comes down to the condition that either $b>0$ or $a<0$, which is exactly the condition given by Kashiwara and Vergne.

If $\omega \otimes \pi^{D}$ has parameter $\left(n_{1}, n_{2}\right)$, then $\omega^{l} \otimes \pi^{D}$ has parameter $\left(-n_{2},-n_{1}\right)$. Thus we can assert more precisely that

4.4.3. Corollary. In Theorem 1 , assume $E=\mathbb{R}, \mathscr{K}=\mathbb{C}, D=D^{\prime}=\mathbb{H}$, and $\varepsilon\left(\pi_{\mathscr{K}} \otimes \omega, \frac{1}{2}\right) \cdot \xi_{\pi}(-1)=1$. Then for fixed $\psi$, exactly one of the spaces $\left\{\Theta_{\psi}\left(\omega \otimes \pi^{D}\right), \Theta_{\psi}\left(\omega^{l} \otimes \pi^{D}\right)\right\}$ does not vanish, and exactly one of the spaces $\left\{\Theta_{\psi}\left(\omega \otimes \pi^{D}\right), \Theta_{\psi^{-}}\left(\omega \otimes \pi^{D}\right)\right\}$ does not vanish.

Of course, the $\varepsilon$-factor criterion does not distinguish between $\omega \otimes \pi^{D}$, and $\omega^{l} \otimes \pi^{D}$, which are $L$-indistinguishable for the $L$-function $L\left(\pi_{\mathscr{H}} \otimes \omega, s\right)$, which is also the $L$-function attached to the representation $\left(\pi_{\mathscr{K}} \otimes \omega\right)_{U}$ of $U_{\mathscr{K}}(D)$ and the standard representation of its $L$-group (cf. $\S 5$, below). At least in the case of classical groups over $\mathbb{R}$, numerous examples of Adams and $\mathrm{Li}$, among others, suggest that the vanishing of a theta lift is highly sensitive to the individual members of an $L$-packet. There is reason to believe that this is also true over nonarchimedean fields.

4.4.4. We now return to the global setting of the previous sections. Thus $E$ is a totally real field, $\mathscr{K}$ a CM quadratic extension, $\pi$ is an automorphic cuspidal representation of $G L(2, E)$, and $D$ and $D^{\prime}$ are two quaternion algebras over $E$ which split over $\mathscr{K}$. In our global applications, we will only be lifting from $G U_{\mathscr{K}}\left(D^{\prime}\right)$ to $G U_{\mathscr{K}}(D)$ when both $D$ and $D^{\prime}$ are division algebras, $\Sigma(D) \supset \Sigma\left(D^{\prime}\right)$, and where $D$ and $D^{\prime}$ have no finite places of ramification in common. At finite places $v$ or places in $\Sigma\left(D^{\prime}\right)$, it follows from the previous discussion that the nonvanishing of $\Theta_{\psi_{v}}\left(\omega_{v} \otimes \pi_{v}^{D^{\prime}}\right)$ entails the nonvanishing of $\Theta_{\psi_{v}^{\prime}}\left(\omega_{v} \otimes \pi_{v}^{D^{\prime}}\right)$ for any $\psi_{v}^{\prime}$; this is because the reduced norm from $D_{v}^{\prime, \times}$ to $E_{v}^{\times}$ is surjective in these cases.

At $v \in \Sigma(D)-\Sigma\left(D^{\prime}\right)$, the methods of $\S 4.2-3$ show again that the vanishing of $\Theta_{\psi_{v}}\left(\omega_{v} \otimes \pi_{v}^{D^{\prime}}\right)$ is independent of the choice of $\psi_{v}$, but now the image of the reduced norm from $D_{v}^{\prime, \times}$ to $E_{v}^{\times} \cong \mathbb{R}^{\times}$is of index two. Thus $\omega_{v}^{-1} \otimes \check{\pi}_{v}^{D}=$ $\Theta_{\psi_{v}}\left(\omega_{v} \otimes \pi_{v}^{D^{\prime}}\right) \oplus \Theta_{\left(\psi_{v}\right)^{-}}\left(\omega_{v} \otimes \pi_{v}^{D^{\prime}}\right)$ is the sum of holomorphic and antiholomorphic pieces. We note that replacing $\psi_{v}$ by $\left(\psi_{v}\right)^{-}$is equivalent to replacing $U\left(D^{\prime}\right)$ 
by $U\left(-D^{\prime}\right)$; thus

If the $\psi$-lift of a form from $U\left(D^{\prime}\right)$ to $U(D)$ is holomorphic at $v$, the $\psi$-lift of the corresponding form from $U\left(-D^{\prime}\right)$ to $U(D)$ is antiholomorphic at $v$.

At $v \in \Sigma(D)^{\prime}$, both $D_{v}$ and $D_{v}^{\prime}$ are isomorphic to $\mathbb{H}$, and the vanishing of the local lift $\Theta_{\psi_{v}}\left(\omega_{v} \otimes \pi_{v}^{D^{\prime}}\right)$ is determined by Corollary 4.4.3. For our purposes, it suffices to observe that, for fixed $\psi_{v}$, we can arrange that $\Theta_{\psi_{v}}\left(\omega_{v} \otimes \pi_{v}^{D^{\prime}}\right) \neq 0$ by replacing $\omega_{v}$ by $\omega_{v}^{l}$. In this context, the main theorem of [10] has the following immediate consequence.

4.4.5. Corollary. Assume that $\Sigma\left(D^{\prime}\right) \subset \Sigma(D)$, and that $D$ and $D^{\prime}$ have no finite places of ramification in common. Fix a global additive character $\psi: E_{\mathrm{A}} / E \rightarrow$ $\mathbb{C}^{\times}$. Fix a finite set of primes $T$ of $E$ which do not split in $\mathscr{K}, T \supset \Sigma$, and a character $\chi_{v}$ of $\mathscr{K}_{V}^{\times}$at each $v \in T$, such that

(a) $\varepsilon\left(\pi_{\mathscr{K}, v} \otimes \chi_{v}, \frac{1}{2}\right) \cdot \varepsilon\left(D_{v}\right) \cdot \varepsilon\left(D_{v}^{\prime}\right) \xi_{\pi_{v}}(-1)=1$ for all $v \in T$

(b) $\xi_{\pi_{v}} \cdot \xi_{\chi_{v}}=1$ for all $v \in T$, where $\xi_{\chi_{v}}$ is the restriction of $\chi_{v}$ to $E_{v}^{\times}$.

Possibly after replacing $\chi_{v}$ by $\chi_{v}^{l}$ for some subset of $v \in \Sigma(D)^{\prime}$, there exists an algebraic Hecke character $\omega$ of $\mathscr{K}$, with $\xi_{\omega} \cdot \xi_{\pi}=1$, such that

(i) $\omega_{v}=\chi_{v}$ for all $v \in T$;

(ii) $\Theta_{\psi_{v}}\left(\omega_{v} \otimes \pi_{v}^{D^{\prime}}\right)=\omega_{v}^{-1} \otimes \check{\pi}_{v}^{D} \neq 0$ for all places $v$ of $E$; and

(iii) $L\left(\pi_{\mathscr{H}} \otimes \omega, \frac{1}{2}\right) \neq 0$.

Proof. Let $S$ denote the set of places at which $\varepsilon\left(D_{v}\right) \cdot \varepsilon\left(D_{v}^{\prime}\right)=-1$. Then the cardinality of $S$ is even. The main theorem of [10] asserts the existence of $\omega$ with the prescribed restrictions $\chi_{v}$ at $v \in T$, satisfying (i), (iii), and

$$
\varepsilon\left(\pi_{\mathscr{K}, v} \otimes \omega_{v}, \frac{1}{2}\right) \cdot \varepsilon\left(D_{v}\right) \cdot \varepsilon\left(D_{v}^{\prime}\right) \xi_{\pi_{v}}(-1)=1 \text { for all places } v \text { of } E \text {. }
$$

We have proved in this section that, under the given hypotheses, (4.4.6) is equivalent to condition (ii), except possibly for places in $\Sigma(D)^{\prime}$, where it may be necessary to replace $\omega_{v}$ by $\omega_{v}^{l}$ (equivalently, to replace $\chi_{v}$ by $\chi_{v}^{l}$ ). But condition (c) of Theorem 1 of [10] says precisely that this can be done without changing the conclusions.

The global analogue of Theorem 4.1 is

4.5. Theorem. Suppose (a) $D \neq D^{\prime}$ and (b) $D$ and $D^{\prime}$ have no finite places of ramification in common. Let $\omega$ be a Hecke character of $\mathscr{K}$, with $\xi_{\omega} \cdot \xi_{\pi}=1$, such that

(i) $\varepsilon\left(\pi_{\mathscr{K}, v} \otimes \omega_{v}, \frac{1}{2}\right) \cdot \varepsilon\left(D_{v}\right) \cdot \varepsilon\left(D_{v}^{\prime}\right) \xi_{\pi_{v}}(-1)=1$ for all places $v$ of $E$,

(ii) $L\left(\pi_{\mathscr{K}} \otimes \omega, \frac{1}{2}\right) \neq 0$,

and (if necessary) the additional hypothesis (ii) of Corollary 4.4 .5 at places $v \in$ $\Sigma(D)^{\prime}$. Then the global theta lift

$$
\Theta_{\psi}\left(\omega \otimes \pi^{D^{\prime}}\right):=\Theta_{\psi}\left(G U_{\mathscr{K}}\left(D^{\prime}\right) \rightarrow G U_{\mathscr{K}}(D) ; \omega \otimes \pi^{D^{\prime}}\right)
$$


is nontrivial, and $\widetilde{\boldsymbol{\Theta}}\left(\omega \otimes \pi^{D^{\prime}}\right)=\omega^{-1} \otimes \check{\pi}^{D}$.

The hypothesis $D \neq D^{\prime}$ is unnecessary, and will be removed in forthcoming joint work with Kudla; however, it simplifies the proof in $\S 6$. Provided $\widetilde{\Theta}\left(\omega \otimes \pi^{D^{\prime}}\right)$ is nontrivial, its identification with $\omega^{-1} \otimes \check{\pi}^{D}$ is an immediate consequence of the corresponding local theorem and the multiplicity one theorem for $D^{\times}$. When $D$ or $D^{\prime}$ is split, the proof of nonvanishing follows the local argument given in 4.3. The special value of the $L$-function intervenes by an argument used in [13,10], which in turn is based on ideas of Waldspurger.

The hypotheses and the conclusion are symmetric in $D$ and $D^{\prime}$; thus we may assume $D$ split, and write $G U_{\mathscr{K}}(s)=G U_{\mathscr{K}}(D)$. Returning to the seesaw diagram, we have the following global analogue of (4.3.2):

The space $\Theta^{\prime}\left(\omega \otimes \pi^{D^{\prime}}\right)=\omega^{-1} \otimes \pi \neq\{0\}$ if and only if the linear form

$$
L_{\omega}: \pi^{D^{\prime}} \rightarrow \mathbb{C} ; \quad f \mapsto L_{\omega}(f)=\int_{E_{\mathbf{A}}^{\times} \cdot \mathscr{K}^{\times} \backslash \mathscr{K}_{\mathbf{A}}^{\times}} f(x) \cdot \omega(x) d x
$$

is nontrivial.

Now the global theta lift of $\S 3.8$ from $G L(2)$ to $G O\left(D^{\prime}\right)$ defines maps

$$
\theta_{\varphi}: \pi \rightarrow \check{\pi}^{D^{\prime}} \otimes \pi^{D^{\prime}}, \quad \varphi \in \mathscr{S}\left(D^{\prime}(\mathbf{A})\right)
$$

see $[13, \S 7]$ for details. By $[10,(2.3 .11)]$, there exist $\check{f} \in \check{\pi}^{D^{\prime}}, f \in \pi^{D^{\prime}}, c \in \mathbb{C}^{\times}$ such that

$$
L_{\omega^{-1}}(\check{f}) \cdot L_{\omega}(f)=\left.c \cdot L\left(\pi_{\mathscr{K}} \otimes \omega, \frac{1}{2}\right) \cdot \prod_{v} \Lambda_{v}(s)\right|_{s=0} \cdot
$$

Here $\check{f} \otimes f=\theta_{\varphi}(F)$ for some $F \in \pi$ and $\varphi \in \mathscr{S}\left(D^{\prime}(\mathbf{A})\right), c$ is a product of local Euler factors and a volume, and $\Lambda_{V}(s)$ are local zeta integrals, depending on $\check{f}, f$, and $\varphi$, of the type studied in Jacquet's work on the Rankin-Selberg convolution. We write $\Lambda_{v}(0)=\left.\Lambda_{v}(s)\right|_{s=0}$. It thus remains to be shown that we can find local data for $\check{f}, f$, and $\varphi$, and for all $v$, such that the $\Lambda_{v}(0) \neq 0$.

We use the notation of $\S 2$ of [10] without comment. For $v$ split in $\mathscr{K}$, the local Weil representation for the pair $(G L(2), G O(\mathscr{K}))$ extends to an action of $G L\left(2, E_{v}\right)$; in other words, the $v$-component of the subgroup $G\left(\varepsilon_{\mathscr{K}}\right)^{1} \subset$ $G L\left(2, E_{\mathbf{A}}\right)$ is all of $G L\left(2, E_{v}\right)$. It follows that, as $\varphi$ varies, the local data denoted $W^{\psi_{v}}\left(\varphi_{2}, \omega\right)$ and $\Phi_{1, v}$ in the integral (2.3.8) defining $\Lambda_{v}(s)$ are arbitrary in the split case. We can then appeal to Jacquet's results to conclude that, for an appropriate choice of local data at $v$, the local zeta integral does not vanish at $s=0$.

Now suppose $v$ does not split in $\mathscr{K}$. Then the proof of the main theorem of [10] (especially (1.4.9.1)) shows that we can find a Hecke character $\omega^{\prime}$, with $\omega_{v}^{\prime}=\omega_{v}$, such that

$$
L_{\omega^{\prime-1}}\left(\check{f}^{\prime}\right) \cdot L_{\omega^{\prime}}\left(f^{\prime}\right) \neq 0 \text { for some } \check{f}^{\prime} \in \check{\pi}^{D^{\prime}}, f \in \pi^{D^{\prime}} .
$$


Since none of the factors in (4.5.2) has a pole at $s=0$ (cf. [10, 2.3.12]), it follows that none of them equals zero. But the local factors at $v$ for $\omega^{\prime}$ and $\omega$ are the same.

This completes the proof of Theorem 4.5 when $D$ or $D^{\prime}$ is split. The proof of nonvanishing in the remaining cases is based on Rallis' doubling method, and will be given in $\S 6$.

4.5.3. Remark. (i) Although the local Howe duality conjecture (cf. $\S 3.7$ ) is not known in general in residue characteristic two, [35] and the arguments of $\S 4.3$ show that it is valid for the pair $\left(G U_{\mathscr{K}}\left(D^{\prime}\right), G U_{\mathscr{K}}(D)\right)$ when $D$ or $D^{\prime}$ is split. Since under our hypotheses this is true locally everywhere, we use local Howe duality without comment in what follows.

(ii) Let $\left(G, G^{\prime}\right)$ be any dual reductive pair which verifies local Howe duality at every place. Let $\pi$ be an irreducible automorphic representation of $G$. A familiar argument of Rallis [52] shows that, if $\Theta\left(G \rightarrow G^{\prime} ; \pi\right)$ is a space of cusp forms, then it is irreducible. This applies in particular to the pair $\left(G U_{\mathscr{K}}(D), G U_{\mathscr{K}}\left(D^{\prime}\right)\right)$ when $D^{\prime}$ is a division algebra.

4.6. We will need one more local result. Let $V$ be a one-dimensional hermitian space over $\mathscr{K}$, and let $\omega$ be a character of $G U_{\mathscr{K}}(V) \cong \mathscr{K}^{\times}$. We consider a Schrödinger model for the pair $\left(G U_{\mathscr{K}}(V), G U_{\mathscr{K}}(s)\right)$ acting on $\mathscr{S}(V)$, and obtain a representation $\Theta(\omega)=\Theta\left(G U_{\mathscr{K}}(V) \rightarrow G U_{\mathscr{K}}(s) ; \omega\right)$ of $G U_{\mathscr{K}}(s)_{+}$(notation of (3.7.1)). This model depends on the choice of a splitting, as in $\S 3.6$. However, the restriction of $\Theta(\omega)$ to $G L(2, E)_{+} \subset G U_{\mathscr{K}}(s)_{+}$is independent of the splitting, as one sees from the seesaw diagram (3.5.1.1), with $W^{\prime}=$ any two-dimensional symplectic space. Indeed, we can apply Lemma 3.7.4 and 3.7.8 (since $U_{\mathscr{K}}(V)$ is compact) to deduce that, for any irreducible representation $\sigma$ of $G L(2, E)_{+}$,

$$
\operatorname{Hom}_{G L(2, E)_{+}}(\sigma \otimes \Theta(\omega), \mathbf{C}) \cong \operatorname{Hom}_{G U_{\mathscr{H}}(V)}(\boldsymbol{\Theta}(\sigma) \otimes \omega, \mathbf{C})
$$

where $\Theta(\sigma)=\Theta\left(G L(2, E) \rightarrow G O\left(R_{\mathscr{K} / E} V\right) ; \sigma\right)$. Let $\alpha: G L(2, E) \rightarrow \mathbb{C}^{\times}$denote the character $\|\cdot\| \circ$ det . Now the right-hand side of $(4.6 .1)$ is clearly trivial unless $\check{\sigma} \otimes \alpha^{1 / 2}$ is a constituent of the representation $\pi(\omega, \mathscr{K})$ of Proposition 1.8.2, restricted to $G L(2, E)_{+}$. Indeed, the proof of that proposition (cf. [17, $\S 4])$ identifies

$$
\pi(\omega, \mathscr{K}) \cong \widetilde{\Theta}\left(G O\left(R_{\mathscr{K} / E} V\right) \rightarrow G L(2, E) ; \omega^{\prime}\right) \otimes \alpha^{1 / 2},
$$

where $\omega^{\prime}$ is a certain irreducible representation of $G O\left(R_{\mathscr{K} / E} V\right)$ whose restriction to $G U_{\mathscr{K}}(V)$ contains $\omega$. Thus

4.6.3. Lemma. Under the above hypotheses, $\Theta(\omega) \otimes \alpha^{1 / 2}$ is an irreducible constituent of $\pi(\omega, \mathscr{K})$, restricted to $G L(2, E)_{+}$.

The irreducibility, which is an issue at most in residue characteristic two, can be deduced from the seesaw diagram (3.5.1.1) and the proof of (4.6.2) in [17, loc.cit.]. 


\section{RATIONALITY PROPERTIES OF THE THETA CORRESPONDENCE}

5.1. Henceforward, $D$ and $D^{\prime}$ are both division algebras, $D \neq D^{\prime}$. Assume as before that $\pi^{D^{\prime}} \otimes \omega$ is attached to automorphic forms on $G U_{\mathscr{K}}\left(D^{\prime}\right)$ of type $(\underline{k}, r, \chi)$, i.e., to holomorphic sections of $\mathscr{E}_{(\underline{k}, r, \chi)}^{D^{\prime}}$. We have seen in the remarks following Theorem 4.5 that, if $\widetilde{\Theta}\left(\omega \otimes \pi^{D^{\prime}}\right) \neq 0$, then $\widetilde{\Theta}\left(\omega \otimes \pi^{D^{\prime}}\right)=\omega^{-1} \otimes \check{\pi}^{D}$. In other words, given any functions $f^{D^{\prime}} \in \pi^{D^{\prime}}$ and $\check{f}^{D} \in \check{\pi}^{D}$, there exists a Schwartz function $\varphi$ such that $\theta_{\varphi}\left(\omega \otimes f^{D^{\prime}}\right)=\omega^{-1} \otimes \check{f}^{D}$.

It is more convenient for us to work in the doubled picture, as in Lemma 3.3; it is also easiest to work with $H_{\mathscr{K}} \times H_{\mathscr{K}}$-eigenvectors at infinity. Thus fix $\chi^{D} \in X\left(\rho_{(\underline{k}, r)}^{D}\right), \chi^{D^{\prime}} \in X\left(\rho_{(\underline{k}, r)}^{D^{\prime}}\right)$, in the notation of $\S 2.2$. Let

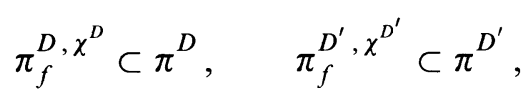

be the subspaces of automorphic forms defined in Lemma 2.4.7. Then $\pi_{f}^{D, \chi^{D}} \cong$ $\pi_{f}^{D}$ and $\pi_{f}^{D^{\prime}}, \chi^{D^{\prime}} \cong \pi_{f}^{D^{\prime}}$ as $D^{\times}\left(\mathbf{A}^{f}\right)$-modules. Let $\pi_{f}^{D, \chi^{D}}(\overline{\mathbb{Q}})$, and $\pi_{f}^{D^{\prime}, \chi^{D^{\prime}}}(\overline{\mathbb{Q}})$ be the $D^{\times}\left(\mathbf{A}^{f}\right)$-invariant $\overline{\mathbb{Q}}$-rational structures on $\pi_{f}^{D, \chi^{D}}$ and $\pi_{f}^{D^{\prime}, \chi^{D^{\prime}}}$, respectively, introduced in Lemma 2.4.7. Fix $f\left(\chi^{D}\right) \in \pi_{f}^{D, \chi^{D}}(\overline{\mathbb{Q}}), f\left(\chi^{D^{\prime}}\right) \in \pi_{f}^{D^{\prime}, \chi^{D^{\prime}}}(\overline{\mathbb{Q}})$. Define the operator $\mathscr{J}(\mathscr{K})$ as in $\S 2.4$, and let

$$
\begin{aligned}
& \check{f}\left(\chi^{D}\right)=f\left(\chi^{D}\right)^{\mathscr{F}(\mathscr{K})} \otimes \xi_{\pi}^{-1} \in\left(\pi_{f}^{D, \chi^{D}}(\overline{\mathbb{Q}})^{\mathscr{F}(\mathscr{K})}\right)^{\vee}, \\
& \check{f}\left(\chi^{D^{\prime}}\right)=f\left(\chi^{D^{\prime}}\right)^{\mathcal{F}(\mathscr{K})} \otimes \xi_{\pi}^{-1} \in\left(\pi_{f}^{D^{\prime}, \chi^{D^{\prime}}}(\overline{\mathbb{Q}})^{\mathcal{F}(\mathscr{K})}\right)^{\vee} .
\end{aligned}
$$

The double of the theta correspondence of Theorem 4.5 can be realized by using Schwartz-Bruhat functions in $\mathscr{S}\left(D(\mathbf{A})^{2}\right)$, as in the proof of Lemma 3.3. We would like to let

$$
\begin{aligned}
& {\left[\omega \otimes \pi^{D^{\prime}}\right](2)=\left[\omega \otimes \pi^{D^{\prime}}\right] \otimes\left[\omega^{-1} \otimes \check{\pi}^{D^{\prime}}\right],} \\
& {\left[\omega^{-1} \otimes \check{\pi}^{D}\right](2)=\left[\omega^{-1} \otimes \check{\pi}^{D}\right] \otimes\left[\omega \otimes \pi^{D}\right] .}
\end{aligned}
$$

These are to be viewed as automorphic representations of

$$
G^{\prime}:=G\left(U_{\mathscr{K}}\left(D^{\prime}\right) \times U_{\mathscr{H}}\left(-D^{\prime}\right)\right) \text { and } G:=G\left(U_{\mathscr{H}}(D) \times U_{\mathscr{K}}(D)\right),
$$

respectively. In most cases, this is sufficient, but when $\pi=\pi(\eta, \mathscr{L})$ is a space of binary theta functions attached to a Hecke character $\eta$ of a quadratic $\mathrm{CM}$ extension $\mathscr{L}$ of $E$, the spaces defined in (5.1.2) decompose as infinite sums of $L$-indistinguishable irreducible representations of $G^{\prime}$ and $G$, respectively, indexed by sets of places of $E$ (cf. [25] and Proposition 1.8.2). This is not too serious, since the theta lift from $G^{\prime}$ to $G$ of any of these components is again irreducible, by Remark 4.5.3(ii). We let $\left[\omega \otimes \pi^{D^{\prime}}\right](2),\left[\omega^{-1} \otimes \check{\pi}^{D}\right](2)$ denote irreducible components of the spaces defined in (5.1.2). Later we will make 
more precise choices in order to specify the holomorphy type of the component chosen, and to guarantee nonvanishing of certain linear forms (cf. the remarks following (5.2.1) and (5.3.6)).

Let $\left[\omega \otimes f\left(\chi^{D^{\prime}}\right)\right](2)=\left[\omega \otimes f\left(\chi^{D^{\prime}}\right)\right] \otimes\left[\omega^{-1} \otimes \check{f}\left(\chi^{D^{\prime}}\right)\right] \in\left[\omega \otimes \pi^{D^{\prime}}\right](2)$, and define $\left[\omega^{-1} \otimes \check{f}\left(\chi^{D}\right)\right](2) \in\left[\omega^{-1} \otimes \check{\pi}^{D}\right](2)$ analogously. For brevity, we write $F^{D^{\prime}}=\left[\omega \otimes f\left(\chi^{D^{\prime}}\right)\right](2), F^{D}=\left[\omega^{-1} \otimes \check{f}\left(\chi^{D}\right)\right](2)$, when there is no danger of confusion. As above, we let $\left[\omega \otimes \pi_{f}^{D^{\prime}}, \chi^{D^{\prime}}\right](2)$ and $\left[\omega^{-1} \otimes \check{\pi}_{f}^{D, \chi^{D}}\right](2)$ denote the $G^{\prime}\left(\mathbf{A}^{f}\right)$ - and $G\left(\mathbf{A}^{f}\right)$-subrepresentations spanned by $F^{D^{\prime}}$ and $F^{D}$, respectively. Then the $G^{\prime}\left(\mathbf{A}^{f}\right)$ - (resp. $G\left(\mathbf{A}^{f}\right)$-) translates of $F^{D^{\prime}}$ (resp. $F^{D}$ ) generate an invariant $\overline{\mathbb{Q}}$-structure $\left[\omega \otimes \pi_{f}^{D^{\prime}}, \chi^{D^{\prime}}\right](2)(\overline{\mathbb{Q}})$ (resp. $\left.\left[\omega^{-1} \otimes \check{\pi}_{f}^{D, \chi^{D}}\right](2)(\overline{\mathbb{Q}})\right)$.

Now $L\left(\check{\pi}_{\mathscr{K}} \otimes \omega^{-1}, s\right)=L\left(\pi_{\mathscr{K}} \otimes \omega, s\right)$, under our hypothesis $\xi_{\pi} \cdot \xi_{\omega}=1$. Assuming Theorem 4.5, there thus exists $\varphi_{0} \in \mathscr{S}\left(D(\mathbf{A})^{2}\right)$ such that

$$
\theta_{\varphi_{0}}\left(F^{D^{\prime}}\right)=F^{D}
$$

Here we are using the remarks in $\S 4.4 .4$ to identify the archimedean constituents of $\Theta_{\psi}\left(G^{\prime} \rightarrow G ; \omega \otimes \pi^{D^{\prime}}\right)$; specifically, it suffices to recall that, for $v \in \Sigma\left(D^{\prime}\right)$, $\check{f}\left(\chi^{D}\right)$ is antiholomorphic and $f\left(\chi^{D}\right)$ is holomorphic at $v$.

Since both $F^{D^{\prime}}$ and $F^{D}$ are of pure $\left(H_{\mathscr{K}} \times H_{\mathscr{K}} \times H_{\mathscr{K}} \times H_{\mathscr{K}}\right)(\mathbb{R})$-type, we may assume $\varphi_{0}$ to be of the form $\varphi_{\infty} \otimes \varphi_{f}$, where $\varphi_{\infty} \in \mathscr{S}\left(D(\mathbb{R})^{2}\right)$, $\varphi_{f} \in \mathscr{S}\left(D\left(\mathbf{A}^{f}\right)^{2}\right)$. Howe's theory of minimal $K$-types [15] even allows us to pick an optimal $\varphi_{\infty}$, determined uniquely, up to scalar multiples, by the property of being of pure $H_{\mathscr{K}}(\mathbb{R})^{8}$ type (four copies of $H_{\mathscr{K}}(\mathbb{R})$ for $G^{\prime}$, four copies for $G$ ), by property (5.1.3), and by the condition of being of minimal degree in the (polynomial) Fock model. (This is actually completely trivial except at places for which $D^{\prime}$ and $D$ are both split.) Since we will not need to compute archimedean integrals, it is not important which $\varphi_{\infty}$ we choose; but let $\mathscr{S}\left(D(\mathbb{R})^{2}\right)(\underline{k}, r, \chi)$ denote the (one-dimensional) complex vector space spanned by some $\varphi_{\infty}$, which is minimal in Howe's sense. It is not difficult to write down $\varphi_{\infty}$ explicitly in terms of majorants.

Now let $\mathscr{S}\left(D\left(\mathbf{A}^{f}\right)^{2}\right)\left(\mathbb{Q}^{\mathrm{ab}}\right)$ denote the space of $\mathbb{Q}^{\mathrm{ab}}$-valued Schwartz-Bruhat functions on $D\left(\mathbf{A}^{f}\right)^{2}$, where $\mathbb{Q}^{\text {ab }}$ is the field obtained by adjoining all roots of unity to $\mathbb{Q}$. Let $\mathscr{S}\left(\mathbb{Q}^{\mathrm{ab}}\right)=\mathscr{S}\left(D(\mathbf{A})^{2}\right)(\underline{k}, r, \chi)\left(\mathbb{Q}^{\mathrm{ab}}\right)=\varphi_{\infty} \otimes \mathscr{S}\left(D\left(\mathbf{A}^{f}\right)^{2}\right)\left(\mathbb{Q}^{\mathrm{ab}}\right)$, $\mathscr{S}(\overline{\mathbb{Q}})=\mathscr{S}\left(\mathbb{Q}^{\mathrm{ab}}\right) \otimes \overline{\mathbb{Q}}$. The oscillator representation of the big metaplectic group $\operatorname{Mp}\left(\mathscr{W}^{\prime \prime}\right)\left(\mathbf{A}^{f}\right)$ of Lemma 3.3 preserves $\mathscr{S}\left(\mathbb{Q}^{\mathrm{ab}}\right)$; this is clear by the standard formulas for the Schrödinger model, which involve only Fourier transform, translation of variables, and multiplication by values of abelian characters or characters of second degree. The construction of the splittings in Lemma 3.3 then shows that $G\left(\mathbf{A}^{f}\right)$ and $G^{\prime}\left(\mathbf{A}^{f}\right)$ also preserve $\mathscr{S}\left(\mathbb{Q}^{\mathrm{ab}}\right)$. It should be remarked that replacing the polarization of the big symplectic vector space $\mathscr{W}^{\prime \prime}$ by another polarization respects the spaces of $\mathbb{Q}^{\mathrm{ab}}$-valued Schwartz-Bruhat 
functions, since the operation is again carried out by means of translation of variables, multiplication by values of abelian characters, and partial Fourier transform.

In particular, the kernel of the map (cf. (3.7.2))

$$
\begin{aligned}
& {\left[\omega \otimes \pi_{f}^{D^{\prime}, \chi^{D^{\prime}}}\right](2)(\overline{\mathbb{Q}}) \otimes_{\mathbb{Q}^{\mathrm{ab}}} \mathscr{S}\left(\mathbb{Q}^{\mathrm{ab}}\right)} \\
& \rightarrow\left[\left[\omega \otimes \pi_{f}^{D^{\prime}, \chi^{D^{\prime}}}\right](2)(\overline{\mathbb{Q}}) \otimes_{\mathbb{Q}^{\mathrm{ab}}} \mathscr{S}\left(\mathbb{Q}^{\mathrm{ab}}\right)\right]_{\left(U_{\mathscr{H}}\left(D^{\prime}\right) \times U_{\mathscr{H}}\left(-D^{\prime}\right)\right)\left(\mathbf{A}^{f}\right)}
\end{aligned}
$$

is defined over $\overline{\mathbb{Q}}$ (even over $\mathbb{Q}^{\mathrm{ab}} \cdot \mathbb{Q}\left(\pi, D^{\prime}\right) \cdot \mathbb{Q}(\pi, D) \cdot E_{\Psi^{\prime}} \cdot E_{\Psi} \cdot E\left(\chi^{D}\right) \cdot E\left(\chi^{D^{\prime}}\right)$ ). It follows that

$$
\begin{aligned}
& {\left[\omega^{-1} \otimes \check{\pi}_{f}^{D, \chi^{D}}\right](2)^{\theta}:=\left\{\theta_{\varphi}\left(F^{D^{\prime}}\right), \varphi \in \mathscr{S}(\overline{\mathbb{Q}})\right\} \text { is a } G\left(\mathbf{A}^{f}\right) \text { - }} \\
& \text { invariant } \overline{\mathbb{Q}} \text {-structure on }\left[\omega^{-1} \otimes \check{\pi}_{f}^{D, \chi^{D}}\right](2) .
\end{aligned}
$$

But we already have a $G\left(\mathbf{A}^{f}\right)$-invariant $\overline{\mathbb{Q}}$-structure on the latter space, namely $\left[\omega^{-1} \otimes \check{\pi}_{f}^{D, \chi^{D}}\right](2)(\overline{\mathbb{Q}})$. Since the representation of $G\left(\mathbf{A}^{f}\right)$ on this space is irreducible, it follows from Schur's lemma that there is a constant $c\left(\varphi_{\infty}, \omega \otimes \pi, D^{\prime}, D\right) \in \mathbb{C}^{\times}$such that

$$
\left[\omega^{-1} \otimes \check{\pi}_{f}^{D, \chi^{D}}\right](2){ }^{\theta}=c\left(\varphi_{\infty}, \omega \otimes \pi, D^{\prime}, D\right) \cdot\left[\omega^{-1} \otimes \check{\pi}_{f}^{D, \chi^{D}}\right](2)(\overline{\mathbb{Q}}) .
$$

5.2. In order to compare the constant $c\left(\varphi_{\infty}, \omega \otimes \pi, D^{\prime}, D\right)$ with something we understand, we introduce a linear form on $\left[\omega^{-1} \otimes \check{\pi}_{f}^{D, \chi^{D}}\right](2)$. Namely, the diagonal subgroup

$$
Z_{G}(\mathbf{A})=\left\{(z, z) \in G U_{\mathscr{K}}(D)(\mathbf{A}) \times G U_{\mathscr{K}}(D)(\mathbf{A}) \mid z \in \mathscr{K}_{\mathbf{A}}^{\times}\right\} \subset G(\mathbf{A})
$$

acts trivially on $\left[\omega^{-1} \otimes \check{\pi}_{f}^{D, \chi^{D}}\right](2)$, by construction, and we can define

$$
B_{D}(F)=\int_{Z_{G}(\mathbf{A}) \cdot G U_{\mathscr{R}}(D)(\mathbb{Q}) \backslash G U_{\mathscr{K}}(D)(\mathbf{A})} F(g) d g,
$$

where the integral is computed along the diagonally embedded $G U_{\mathscr{K}}(D)(\mathbf{A}) \subset$ $G(\mathbf{A})$. Given our choice of $F^{D}$ it is easy to see that-possibly after changing the irreducible component $\left[\omega^{-1} \otimes \check{\pi}^{D}\right](2)$ in the case of binary theta functionsthe linear form $B_{D}$ is not identically zero. Indeed, we may take $f\left(\chi^{D}\right) \in$ $\pi^{D}\left(\left\{\chi_{v}\right\}\right)^{\text {new }}$, as in $\S 2.4$. Then Lemma 2.4 .5 implies that $\check{f}\left(\chi^{D}\right)$ is a nonzero algebraic multiple of $\overline{f\left(\chi^{D}\right)}$, and $B_{D}\left(F^{D}\right)$ is a nonzero multiple of the (square of the) $L_{2}$-norm of $f\left(\chi^{D}\right)$. It thus follows from 2.4.5 and 2.5.6 that

$$
B_{D}(F) \sim q^{D}(\pi) \quad \text { for any } F \in\left[\omega^{-1} \otimes \check{\pi}_{f}^{D, \chi^{D}}\right](2)(\overline{\mathbb{Q}}) .
$$

Our first relation is then

5.2.3. Lemma. For any $\varphi \in \mathscr{S}(\overline{\mathbb{Q}}), q^{D}(\pi) \cdot c\left(\varphi_{\infty}, \omega \otimes \pi, D^{\prime}, D\right) \sim_{\overline{\mathbb{Q}}} B_{D}\left(\theta_{\varphi}\left(F^{D^{\prime}}\right)\right)$.

Using Rallis' doubling method, it will be proved in $\S 6.3$ that 
5.2.4. Proposition. There exists a constant $C_{1}=C_{1}\left(\varphi_{\infty}, \underline{k}, \chi, \Sigma\left(D^{\prime}\right), \Sigma(D)\right) \in$ $\mathbb{C}^{\times}$such that, for all $\varphi \in \mathscr{S}(\overline{\mathbb{Q}})$,

$$
B_{D}\left(\theta_{\varphi}\left(F^{D^{\prime}}\right)\right) \sim_{\bar{Q}} C_{1} \cdot L\left(\pi_{\mathscr{H}} \otimes \omega, \frac{1}{2}\right) \cdot q^{D^{\prime}}(\pi),
$$

where the L-function is given the Jacquet-Langlands normalization, for which $s=\frac{1}{2}$ is the center of symmetry of the functional equation.

5.3. The argument leading up to (5.1.5) works more generally when $D^{\prime}$ is replaced by any hermitian space $V$, including the 1-dimensional hermitian space $V=\mathscr{K}$. Write $D=V_{1} \oplus V_{2}=i(\mathscr{K}) \oplus i(\mathscr{K}) \delta$ as in $\S 3$, a sum of two 1dimensional $\mathscr{K}$-spaces. We will soon have use for the seesaw diagram (3.5.3.2), with $V=D^{\prime}, V^{\prime}=D$ :

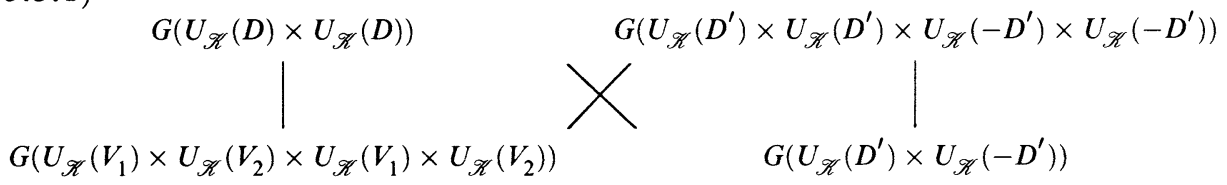

Let $\mathscr{G}$ (resp. $\mathscr{G}^{\prime}$ ) denote the lower left-hand (resp. upper right-hand) group in this diagram. We wish to study the rational structure induced on the representation of $\mathscr{G}^{\prime}\left(\mathbf{A}^{f}\right)$ obtained by theta lift from characters of the abelian group $\mathscr{G}(\mathbb{Q}) \backslash \mathscr{G}(\mathbf{A})$. Thus, let $\eta_{1}$ and $\eta_{2}$ be motivic Hecke characters of $\mathscr{K}$, and consider the character $\eta_{1} \otimes \eta_{2}(2):=\eta_{1} \otimes \eta_{2} \otimes \eta_{1}^{-1} \otimes \eta_{2}^{-1}$ of

$$
\left(\mathscr{K}^{\times} \backslash \mathscr{K}_{\mathbf{A}}^{\times}\right)^{4} \cong\left[G U_{\mathscr{H}}\left(V_{1}\right) \times G U_{\mathscr{H}}\left(V_{2}\right)\right]^{2}(\mathbb{Q}) \backslash\left[G U_{\mathscr{H}}\left(V_{1}\right) \times G U_{\mathscr{H}}\left(V_{2}\right)\right]^{2}(\mathbf{A}),
$$

restricted to $\mathscr{G}(\mathbb{Q}) \backslash \mathscr{G}(\mathbf{A})$. Define $\left[\pi\left(\eta_{1} \otimes \eta_{2}, \mathscr{K}\right)\right](2)$ to be the restriction to $\left(D^{\prime, \times}\right)^{4} \cap \mathscr{G}^{\prime}$ of the automorphic representation $\pi\left(\eta_{1}, \mathscr{K}\right)^{D^{\prime}} \otimes \pi\left(\eta_{2}, \mathscr{K}\right)^{D^{\prime}} \otimes$ $\check{\pi}\left(\eta_{1}, \mathscr{K}\right)^{D^{\prime}} \otimes \check{\pi}\left(\eta_{2}, \mathscr{K}\right)^{D^{\prime}}$ of $\left(D^{\prime, \times}\right)^{4}$.

Consider the space of functions

$$
\pi\left(\eta_{1} \otimes \eta_{2}(2)\right)^{\theta}:=\left\{\theta_{\varphi}\left(\mathscr{G} \rightarrow \mathscr{G}^{\prime} ; \eta_{1} \otimes \eta_{2}(2)\right) \mid \varphi \in \mathscr{S}(\overline{\mathbb{Q}})\right\} .
$$

Here $\mathscr{S}(\overline{\mathbb{Q}}) \subset \mathscr{S}\left(D(\mathbf{A})^{2}\right)$ is the same space used in $\S 5.1$; the definition (5.3.2) makes sense because $\left(\mathscr{G}, \mathscr{G}^{\prime}\right)$ and $\left(G, G^{\prime}\right)$ fit into the seesaw diagram (5.3.1). We may retroactively impose the hypothesis that $\varphi_{\infty}$ is an eigenfunction for a maximal compact connected subgroup $K_{\mathscr{G}} \times K_{\mathscr{G}^{\prime}} \subset \mathscr{G}(\mathbb{R}) \times \mathscr{G}^{\prime}(\mathbb{R})$, chosen compatibly with the maximal compact subgroups of $G(\mathbb{R})$ and $G^{\prime}(\mathbb{R})$. Actually, Howe's theory of minimal $K$-types implies that this is redundant, given that we have already chosen $\varphi_{\infty}$ to be of minimal degree for the pair $G \times G^{\prime}$.

In particular, $\pi\left(\eta_{1} \otimes \eta_{2}(2)\right)^{\theta} \otimes \mathbb{C}$ is a certain $K_{\mathscr{G}^{\prime}}$-type subspace:

$$
\widetilde{\Theta}\left(\mathscr{G} \rightarrow \mathscr{G}^{\prime} ; \eta_{1} \otimes \eta_{2}(2)\right)^{h} \subset \Theta\left(\mathscr{G} \rightarrow \mathscr{G}^{\prime} ; \eta_{1} \otimes \eta_{2}(2)\right) .
$$

This will be made more precise in $\S 5.4$ when we make specific choices of $\eta_{1}$ and $\eta_{2}$. Just as before, we find that $\pi\left(\eta_{1} \otimes \eta_{2}(2)\right)^{\theta}$ is a $\mathscr{G}^{\prime}\left(\mathbf{A}^{f}\right)$-invariant $\overline{\mathbb{Q}}$ form of the space $\widetilde{\Theta}\left(\mathscr{G} \rightarrow \mathscr{G}^{\prime} ; \eta_{1} \otimes \eta_{2}(2)\right)^{h}$. Here we need to be a little bit 
careful, since $\pi\left(\eta_{1} \otimes \eta_{2}(2)\right)^{\theta}$ only becomes a $\mathscr{G}^{\prime}\left(\mathbf{A}^{f}\right)$-module upon choosing a splitting of the metaplectic group, as in $\S 3$. This depends in turn on the choice of a Hecke character $\gamma$ of $\mathscr{K}$, extending the quadratic Hecke character $\varepsilon_{\mathscr{K}}$ of $E$. However, $\gamma$ can be chosen of the form $\gamma^{\prime} \cdot\|\cdot\|_{\mathbf{A}}^{1 / 2}$, where $\gamma^{\prime}$ is an algebraic Hecke character. Such a $\gamma$ certainly takes only algebraic values on $\mathscr{K}_{\mathbf{A}^{f}}^{\times}$, although the values do not lie in a finite extension of $\mathbb{Q}$. With more care, it can be shown how to compensate for the effects of $\gamma$.

5.3.3. Lemma. If $\widetilde{\Theta}\left(\mathscr{G} \rightarrow \mathscr{G}^{\prime} ; \eta_{1} \otimes \eta_{2}(2)\right) \neq 0$, then its restriction to $\left(D^{\prime, \times}\right)^{4} \cap$ $\mathscr{G}^{\prime}$ is a sum of irreducible constituents of $\left[\pi\left(\eta_{1} \otimes \eta_{2}, \mathscr{K}\right)\right](2)$.

By the strong multiplicity one theorem for $D^{\prime, \times}$, any irreducible automorphic representation $\sigma$ of $\left(D^{\prime, \times}\right)^{4} \cap \mathscr{G}^{\prime}$ is a constituent of a unique irreducible automorphic representation of $\left(D^{\prime} \times\right)^{4}$, which is determined by the local constituents of $\sigma$ at almost all places. Thus Lemma 5.3.3 reduces to the following assertion, an immediate consequence of Lemma 4.6.3.

5.3.3 (bis). Lemma. If $\widetilde{\Theta}\left(\mathscr{G} \rightarrow \mathscr{G}^{\prime} ; \eta_{1} \otimes \eta_{2}(2)\right) \neq 0$, then, at almost all places $v$ of $E$, the restriction to $\left(D_{v}^{\prime, \times}\right)^{4} \cap \mathscr{G}^{\prime}\left(E_{v}\right)$ of $\widetilde{\Theta}\left(\mathscr{G}\left(E_{v}\right) \rightarrow \mathscr{G}^{\prime}\left(E_{v}\right) ; \eta_{1, v} \otimes \eta_{2, v}(2)\right)$ is a sum of irreducible constituents of $\left[\pi_{v}\left(\eta_{1, v} \otimes \eta_{2, v}, \mathscr{K}_{v}\right)\right](2)$.

Here the local representations $\left[\pi_{v}\left(\eta_{1, v} \otimes \eta_{2, v}, \mathscr{K}_{v}\right)\right](2)$ are defined by analogy with $\left[\pi\left(\eta_{1} \otimes \eta_{2}, \mathscr{K}\right)\right](2)$.

We can regard $\left[\pi\left(\eta_{1} \otimes \eta_{2}, \mathscr{K}\right)\right](2)$ as a representation associated to a space of motivic holomorphic automorphic forms on $\left(D^{\prime, \times}\right)^{4}$ of multiweight

$$
\left(\underline{k}\left(\eta_{1}\right), 1-w\left(\eta_{1}\right) ; \underline{k}\left(\eta_{2}\right), 1-w\left(\eta_{2}\right) ; \underline{k}\left(\eta_{1}\right), w\left(\eta_{1}\right)-1 ; \underline{k}\left(\eta_{2}\right), w\left(\eta_{2}\right)-1\right) .
$$

Let $\mathscr{G}^{\prime}(2)=G\left(U_{\mathscr{K}}\left(D^{\prime}\right) \times U_{\mathscr{K}}\left(D^{\prime}\right)\right) \subset \mathscr{G}^{\prime}$, where the first (resp. the second) $U_{\mathscr{K}}\left(D^{\prime}\right)$ is embedded diagonally in the first and third factors (resp. the second and fourth factors) of $\mathscr{G}^{\prime}$. Let $Z_{\mathscr{G}^{\prime}}=\left\{(z, z, z, z) \in G U_{\mathscr{K}}(D)^{2}(\mathbf{A}) \times\right.$ $\left.G U_{\mathscr{K}}(-D)^{2}(\mathbf{A}) \mid z \in \mathscr{K}_{\mathbf{A}}^{\times}\right\} \subset \mathscr{G}^{\prime}(\mathbf{A})$. Define a linear form on

$$
\widetilde{\Theta}\left(\mathscr{G} \rightarrow \mathscr{G}^{\prime} ; \eta_{1} \otimes \eta_{2}(2)\right)
$$

in analogy with (5.2.1):

$$
\widetilde{B}_{D^{\prime}}(F)=\int_{Z_{\mathscr{G}^{\prime}}(\mathbf{A}) \cdot \mathscr{G}^{\prime}(2)(\mathbb{Q}) \backslash \mathscr{G}^{\prime}(2)(\mathbf{A})} F\left(g^{\prime}\right) d g^{\prime} .
$$

5.3.5. Lemma. If $\widetilde{\Theta}\left(\mathscr{G} \rightarrow \mathscr{G}^{\prime} ; \eta_{1} \otimes \eta_{2}(2)\right) \neq 0$, then the linear form $B_{D^{\prime}}$ is not identically zero.

Proof. This is a consequence of a general principle regarding theta lifts. Suppose $V$ and $V^{\prime}$ are hermitian spaces over $\mathscr{K}$, and the theta lift from $U(V)$ to $U\left(V^{\prime}\right)$ is defined in terms of a splitting $\gamma$ and an additive character $\psi$, as in $\S 3$. Suppose the theta lift from $U(V) \rightarrow U\left(-V^{\prime}\right)$ is defined in terms of the complex conjugate splitting $\bar{\gamma}$ and the additive character $\psi$. Then

$$
\Theta\left(U(V) \rightarrow U\left(-V^{\prime}\right) ; \bar{\pi}\right)=\bar{\Theta}\left(U(V) \rightarrow U\left(V^{\prime}\right) ; \pi\right),
$$


where ${ }^{-}$denotes complex conjugation acting on the space of automorphic forms. In particular, integration along the diagonally embedded $U\left(V^{\prime}\right)$ defines a nontrivial linear form on

$$
\Theta\left(U(V) \rightarrow U\left(V^{\prime}\right) ; \pi\right) \otimes \Theta\left(U(V) \rightarrow U\left(-V^{\prime}\right) ; \bar{\pi}\right) .
$$

We apply this when $V=\mathscr{K}, V^{\prime}=D^{\prime}$.

It follows that $\widetilde{\Theta}\left(\mathscr{G} \rightarrow \mathscr{G}^{\prime} ; \eta_{1} \otimes \eta_{2}(2)\right)^{h}$, restricted to $\left(D^{\prime, \times}\right)^{4}$, has a nontrivial intersection with the space spanned by functions of the form

$$
f_{1} \otimes f_{2} \otimes\left(\bar{f}_{1} \otimes \xi_{1}^{-1}\right) \otimes\left(\bar{f}_{2} \otimes \xi_{2}^{-1}\right),
$$

where, at each archimedean place, $f_{i}$ is either a holomorphic or an antiholomorphic vector in $\pi\left(\eta_{i}, \mathscr{K}\right)^{D^{\prime}}$ and $\xi_{i}=\left|\xi_{\pi\left(\eta_{i}, \mathscr{K}\right)}\right|, i=1,2$. The holomorphy can also be seen from an analysis of $K$-types, which we carry out in $\S 5.6$. We therefore assume $f_{1}$ holomorphic and $f_{2}$ antiholomorphic at all places in $\Sigma\left(D^{\prime}\right)$; this hypothesis is justified following Table 5.6.2.

Just as in $\S 1$, the theory of arithmetic automorphic forms defines a $\mathscr{G}^{\prime}\left(\mathbf{A}^{f}\right)$ invariant $\overline{\mathbb{Q}}$-structure $\pi\left(\eta_{1} \otimes \eta_{2}(2)\right)(\overline{\mathbb{Q}})$ on $\pi\left(\eta_{1} \otimes \eta_{2}(2)\right)^{\theta} \otimes \mathbb{C}$. Again, we have to take care to choose the splitting character $\gamma$ appropriately. Now $\pi\left(\eta_{1} \otimes \eta_{1}(2)\right)^{\theta} \otimes$ $\mathbb{C}$ is an irreducible $\mathscr{G}^{\prime}\left(\mathbf{A}^{f}\right)$-module. Indeed, since $\mathscr{G}^{\prime}$ is anisotropic, this follows from local Howe duality by Remark 4.5.3(ii); since $U_{\mathscr{H}}(\mathscr{K})$ is a torus, it is easy to verify that local Howe duality is valid for the pair $\left(U_{\mathscr{K}}(\mathscr{K}), U_{\mathscr{K}}(D)\right)$ even in residue characteristic 2 (cf. $[15, \S 6]$ ). Thus the two $\overline{\mathbb{Q}}$-structures $\pi\left(\eta_{1} \otimes \eta_{2}(2)\right)(\overline{\mathbb{Q}})$ and $\pi\left(\eta_{1} \otimes \eta_{2}(2)\right)^{\theta}$ are proportional. Define $c\left(\varphi_{\infty}, \eta_{1}, \eta_{2}, D^{\prime}\right)$ $\in \mathbb{C}^{\times}$to be the constant of proportionality relating these two $\overline{\mathbb{Q}}$-structures:

$$
\pi\left(\eta_{1} \otimes \eta_{2}(2)\right)^{\theta} \sim_{\overline{\mathbb{Q}}} c\left(\varphi_{\infty}, \eta_{1}, \eta_{2}, D^{\prime}\right) \cdot \pi\left(\eta_{1} \otimes \eta_{2}(2)\right)(\overline{\mathbb{Q}}) .
$$

Write $q^{D^{\prime}}\left(\eta_{i}, \mathscr{K}\right)=q^{D^{\prime}}\left(\pi\left(\eta_{i}, \mathscr{K}\right)\right), i=1,2$. The analogue of Lemma 5.2.3 is

5.3.8. Lemma. For any $\varphi \in \mathscr{S}(\overline{\mathbb{Q}})$,

$$
q^{D^{\prime}}\left(\eta_{1}, \mathscr{K}\right) \cdot q^{D^{\prime}}\left(\eta_{2}, \mathscr{K}\right) \cdot c\left(\varphi_{\infty}, \eta_{1}, \eta_{2}, D^{\prime}\right) \sim_{\overline{\mathbb{Q}}} B_{D^{\prime}}\left(\theta_{\varphi}\left(\eta_{1} \otimes \eta_{2}(2)\right)\right) .
$$

The analogue of Proposition 5.2.4 is

5.3.9. Proposition. Let $\tilde{\eta}_{i}$ be the Hecke character $\eta_{i} / \eta_{i}^{l}, \quad i=1,2$. There exists a constant $C_{2}=C_{2}\left(\varphi_{\infty}, \underline{k}\left(\eta_{1}\right), \underline{k}\left(\eta_{2}\right), \Sigma\left(D^{\prime}\right)\right) \in \mathbb{C}^{\times}$such that, for all $\varphi \in \mathscr{S}(\overline{\mathbb{Q}})$,

$$
\widetilde{B}_{D^{\prime}}\left(\theta_{\varphi}\left(\eta_{1} \otimes \eta_{2}(2)\right)\right) \sim_{\overline{\mathbb{Q}}} C_{2} \cdot L_{\mathscr{K}}\left(\tilde{\eta}_{1}, 1\right) \cdot L_{\mathscr{H}}\left(\tilde{\eta}_{2}, 1\right),
$$

where the L-function is given the Jacquet-Langlands normalization, for which $s=\frac{1}{2}$ is the center of symmetry of the functional equation.

The proof, which again uses Rallis' doubling method, is given in $\S 6.4$. 
5.4. The next step is to combine the formulas of $\S 5.3$ with the rationality criteria of $\S 2$ to determine the constant $c\left(\varphi_{\infty}, \omega \otimes \pi, D^{\prime}, D\right)$ of (5.1.5). For this purpose we have to specify the Hecke characters $\eta_{1}$ and $\eta_{2}$ more precisely.

We apply criterion (i) of Corollary 2.3 .3 to a lifted form $\theta_{\varphi}\left(F^{D^{\prime}}\right)$, which we may assume to be decomposable as in (5.1.3):

$$
\theta_{\varphi}\left(F^{D^{\prime}}\right)=c \cdot F^{D}=c \cdot\left(\omega^{-1} \otimes \check{f}\left(\chi^{D}\right)\right) \otimes\left(\omega \otimes f\left(\chi^{D}\right)\right)
$$

with $c=c\left(\varphi_{\infty}, \omega \otimes \pi, D^{\prime}, D\right)$. We assume $\Sigma(D)$ to be nonempty. Now it follows from 2.3.3(i) that, if $\beta \in X\left(\chi^{D}\right)$ is a Hecke character of $\mathscr{K}^{\times}$, then

$$
L_{\omega^{-1}} \otimes L_{\beta}\left(\omega \otimes f\left(\chi^{D}\right)\right) \sim_{\overline{\mathbb{Q}}} p_{\mathscr{K}}(\beta, \Psi) .
$$

Similarly, write $\delta=\delta_{\infty} \cdot \delta_{f}$, as in $\S 2.4$, and let $f\left(\chi^{D}\right)^{\delta_{f}}(g)=f\left(\chi^{D}\right)\left(g \cdot \delta_{f}\right)$, $g \in D^{\times}(\mathbf{A})$. By imitating the proof of Lemma 2.4.5, we find that

$$
L_{\omega} \otimes L_{\beta^{-1}}\left(\omega^{-1} \otimes \check{f}\left(\chi^{D}\right)\right)=L_{\check{\beta}}\left(f\left(\chi^{D}\right)^{\delta_{f}} \otimes \xi_{\pi}^{-1}\right) \sim_{\overline{\mathbb{Q}}} p_{\mathscr{K}}(\check{\beta}, \Psi),
$$

where for any Hecke character $\alpha$ of $\mathscr{K}^{\times}$we let $\check{\alpha}=\left(\alpha^{l}\right)^{-1}$. Combining (5.4.1-3), we find a second formula for $c\left(\varphi_{\infty}, \omega \otimes \pi, D^{\prime}, D\right)$ :

5.4.4. Lemma. For any $\varphi \in \mathscr{S}(\overline{\mathbb{Q}})$ and any $\beta \in X\left(\chi^{D}\right)$,

$$
p_{\mathscr{K}}(\tilde{\beta}, \Psi) \cdot c\left(\varphi_{\infty}, \omega \otimes \pi, D^{\prime}, D\right) \sim_{\overline{\mathbb{Q}}} L_{\omega} \otimes L_{\beta^{-1}} \otimes L_{\omega^{-1}} \otimes L_{\beta}\left(\theta_{\varphi}\left(F^{D^{\prime}}\right)\right),
$$

where $\tilde{\beta}=\beta / \beta^{l}$.

Here we have used the monomial relation (Proposition 1.4(c)):

$$
p_{\mathscr{K}}(\beta, \Psi) \cdot p_{\mathscr{K}}(\check{\beta}, \Psi) \sim_{\overline{\mathbb{Q}}} p_{\mathscr{K}}(\beta \cdot \check{\beta}, \Psi)=p_{\mathscr{K}}(\tilde{\beta}, \Psi) .
$$

Refer to diagram (5.3.1). Consider the composition

$$
\alpha: H_{\mathscr{K}} \times H_{\mathscr{K}}=\mathscr{K}^{\times} \times \mathscr{K}^{\times} \subset \mathscr{K}^{\times} \times D^{\times} \rightarrow G U_{\mathscr{H}}(D),
$$

where the first map is the natural inclusion and the second map is the one defined in $\S 3.1$. Then $\alpha\left(H_{\mathscr{K}} \times H_{\mathscr{K}}\right) \subset G\left(U_{\mathscr{K}}\left(V_{1}\right) \times U_{\mathscr{K}}\left(V_{2}\right)\right)$, and by dimension considerations we see that this inclusion is an equality. The Hecke character $\omega \otimes \beta^{-1} \otimes \omega^{-1} \otimes \beta$ of $\left(H_{\mathscr{K}}\right)^{4}$, used in Lemma 5.4.4, is the restriction to $\mathscr{G}$ of the Hecke character $\eta_{1} \otimes \eta_{2}(2)$ of $\left(\mathscr{K}^{\times}\right)^{4} \cong G U_{\mathscr{K}}\left(V_{1}\right) \times G U_{\mathscr{K}}\left(V_{2}\right) \times G U_{\mathscr{K}}\left(V_{1}\right) \times$ $G U_{\mathscr{K}}\left(V_{2}\right)$ if and only if

$$
\eta_{1} \cdot \eta_{2}=\omega ; \quad\left(\eta_{1} \cdot \eta_{2}^{l}\right)^{-1}=\beta^{-1}
$$

5.5. We can solve (5.4.6) for the weights of $\eta_{1}, \eta_{2}$ in terms of the weights of $\omega$ and $\beta$, which means that it is time to specify the latter. In doing so, we are subject to four constraints:

(5.5.1) for each $i$, the $k_{j}\left(\eta_{i}\right)$ must all have the same parity;

$$
\begin{aligned}
& k_{j}(\omega)>k_{j} \text { for } j \in \Sigma\left(D^{\prime}\right) \amalg \Sigma(D)^{\prime}, k_{j}>k_{j}(\omega) \text { for } j \in \Sigma(D)- \\
& \Sigma\left(D^{\prime}\right) ;
\end{aligned}
$$


(5.5.3) $\quad \beta \in X\left(\chi^{D}\right)$; in particular, $\beta$ is strictly compatible with $\Psi$;

(5.5.4) $\quad w(\omega)=w(\beta)=r$.

Here (5.5.1) ensures that the $\eta_{i}$ are motivic, (5.5.2) is the condition of Corollary 4.4.1 that that local theta lift at $\sigma_{j}$ of $\omega \otimes \pi^{D^{\prime}}$ be nontrivial, and (5.5.3) is necessary in order that the right-hand side of the formula in 5.4.4 not vanish. Finally, (5.5.4) follows from the formula $\xi_{\pi} \cdot \xi_{\omega}=\xi_{\pi} \cdot \xi_{\beta}=1$.

We now specify that $\omega$ be a Hecke character of $\mathscr{K}^{\times}$, with $\xi_{\pi} \cdot \xi_{\omega}=1$, such that

$$
\begin{aligned}
& \underline{k}(\omega)_{j}=k_{j}+3\left(\text { resp. } k_{j}+1, \text { resp. } k_{j}-1\right) \text { for } j \in \Sigma\left(D^{\prime}\right), \\
& \left(\text { resp. } \Sigma(D)^{\prime}, \text { resp. } \Sigma(D)-\Sigma\left(D^{\prime}\right)\right)
\end{aligned}
$$

Here (5.5.5) corresponds to conditions (i) and (ii) of Corollary 4.4.5, with $T=\Sigma$, and (5.5.6) corresponds to condition (iii) of that corollary, which asserts that such $\omega$ do exist; note that the existence of $\pi^{D}$ implies that $k_{i} \geq 2$ for $i \in \Sigma(D)$. We have taken care of (5.5.2).

By (5.5.5) there is a CM type $\Phi$ of $\mathscr{K}$ such that, if $\Psi=\left\{\tau \in \Phi|\tau|_{E} \in \Sigma\left(D^{\prime}\right) \cup\right.$ $\left.\Sigma\left(D^{\prime}\right)\right\}$, then $\omega$ is strictly compatible with $\imath \Psi$. We may assume $\omega$ compatible with $l \Phi$, and say $\omega \in X\left(\chi_{\lambda}\right)$, as in $\S 1.1$. Write $\Phi=\left\{\tau_{j}\right\},\left.\tau_{j}\right|_{E}=\sigma_{j}$. Then (5.5.5) and (5.5.4) translate as $k(\omega)_{j}=\lambda\left(\tau_{j}\right)-\lambda\left(l \tau_{j}\right)+1=k_{j} \pm 1$ or $k_{j}+3$, depending on $j,-\lambda\left(\tau_{j}\right)-\lambda\left(l \tau_{j}\right)=w\left(\chi_{\lambda}\right)=r$. Solving for $\lambda$, we find

$$
\begin{gathered}
\lambda\left(\tau_{j}\right)=\frac{1}{2}\left(k_{j}-r\right)+1, \quad \lambda\left(l \tau_{j}\right)=-\frac{1}{2}\left(k_{j}+r\right)-1 \quad \text { if } j \in \Sigma\left(D^{\prime}\right) \\
\lambda\left(\tau_{j}\right)=\frac{1}{2}\left(k_{j}-r\right), \quad \lambda\left(\imath \tau_{j}\right)=-\frac{1}{2}\left(k_{j}+r\right) \quad \text { if } j \in \Sigma(D)^{\prime}, \\
\lambda\left(\tau_{j}\right)=\frac{1}{2}\left(k_{j}-r\right)-1, \quad \lambda\left(\imath \tau_{j}\right)=-\frac{1}{2}\left(k_{j}+r\right)+1, \quad j \in \Sigma(D)-\Sigma\left(D^{\prime}\right) .
\end{gathered}
$$

We are forced (by (5.5.3)) to take $k(\beta)_{j}=k_{j}+1$ for $j \in \Sigma(D)$, and

$$
0<k(\beta)_{j} \leq k_{j}-1, \quad k(\beta)_{j} \equiv k_{j}-1 \quad(\bmod 2) \quad \text { for } j \in \Sigma(D)^{\prime} .
$$

We specify $\chi^{D}$ so that

$$
k\left(\chi^{D}\right)_{j}=k_{j}-1 \quad(\bmod 2) \text { for } j \in \Sigma(D)^{\prime} .
$$

Any CM type with which $\chi^{D}$ is compatible has to contain $\Psi$, by (5.5.3), and we may assume $\chi^{D}$ to be compatible with the $\Phi$ introduced above. Say $\chi^{D}=\chi_{\lambda^{D}}$. We can solve for $\lambda^{D}$ as before, and we find that

$$
\begin{array}{cl}
\lambda^{D}\left(l \tau_{j}\right)=\frac{1}{2}\left(k_{j}-r\right), & \lambda^{D}\left(\tau_{j}\right)=-\frac{1}{2}\left(k_{j}+r\right), \quad j \in \Sigma(D) ; \\
\lambda^{D}\left(l \tau_{j}\right)=\frac{1}{2}\left(k_{j}-r\right)-1, & \lambda^{D}\left(\tau_{j}\right)=-\frac{1}{2}\left(k_{j}+r\right)+1, \quad j \in \Sigma(D)^{\prime} .
\end{array}
$$

Finally, say $\eta_{i} \in X\left(\chi_{\lambda_{i}}\right), 1=1,2$. Then (5.4.6) translates as

$$
\lambda_{1}(\tau)+\lambda_{2}(\tau)=\lambda, \quad \lambda_{1}(\tau)+\lambda_{2}(l \tau)=\lambda^{D}, \quad \forall \tau \in \Sigma
$$


Combining (5.5.7), (5.5.9), and (5.5.10), we conclude:

(5.5.11)

$$
\begin{gathered}
\lambda_{1}\left(\tau_{j}\right)-\lambda_{1}\left(l \tau_{j}\right)=1, \quad \lambda_{2}\left(\tau_{j}\right)-\lambda_{2}\left(l \tau_{j}\right)=k_{j}+1, \quad j \in \Sigma\left(D^{\prime}\right) ; \\
\lambda_{1}\left(\tau_{j}\right)-\lambda_{1}\left(l \tau_{j}\right)=-1, \quad \lambda_{2}\left(\tau_{j}\right)-\lambda_{2}\left(l \tau_{j}\right)=k_{j}-1, \quad j \in \Sigma(D)-\Sigma\left(D^{\prime}\right) ; \\
\lambda_{1}\left(\tau_{j}\right)-\lambda_{1}\left(l \tau_{j}\right)=1, \quad \lambda_{2}\left(\tau_{j}\right)-\lambda_{2}\left(l \tau_{j}\right)=k_{j}-1, \quad j \in \Sigma(D)^{\prime} .
\end{gathered}
$$

Let $\Psi^{\prime}=\left\{\tau \in \Psi|\tau|_{E} \in \Sigma\left(D^{\prime}\right)\right\}$. The weights $w\left(\eta_{i}\right)$ are not determined uniquely; we have only specified the restriction $\eta_{1} \otimes \eta_{2}(2)$ to $\mathscr{G}$. However, we can conclude from (5.5.11) that

$$
\begin{aligned}
& k\left(\eta_{1}\right)_{j}=2 \text { for all } j, \eta_{1} \text { is of type }\left(\Psi-\Psi^{\prime}\right) \amalg \imath \Psi^{\prime} \amalg l(\Phi-\Psi) ; \\
& k\left(\eta_{2}\right)_{j}=k_{j}+2 \text { for } j \in \Sigma\left(D^{\prime}\right),=k_{j} \text { for } j \in \Sigma\left(D^{\prime}\right)^{\prime}, \text { and } \eta_{2} \text { is } \\
& \text { of type } \iota \Phi \text {. }
\end{aligned}
$$

We have incidentally verified (5.5.1). The choice of $w\left(\eta_{i}\right) \equiv k\left(\eta_{i}\right)+1$ $(\bmod 2)$, subject to the condition $w\left(\eta_{1}\right)+w\left(\eta_{2}\right)=w(\omega)=r$ (5.4.6), plays no role in what follows.

5.6. We can use the seesaw reciprocity formula 3.8 .4 for diagram (5.3.1) to interpret the right-hand side of the formula in Lemma 5.4.4 as an integral over $G^{\prime}$. Write $L_{\left(\omega, \beta^{-1}\right)}(\cdot)$ instead of $L_{\omega} \otimes L_{\beta^{-1}} \otimes L_{\omega^{-1}} \otimes L_{\beta}(\cdot)$. The result is

$$
L_{\left(\omega, \beta^{-1}\right)}\left(\theta_{\varphi}\left(F^{D^{\prime}}\right)\right)=\int_{Z_{G^{\prime}}(\mathbf{A}) \cdot G^{\prime}(\mathbb{Q}) \backslash G^{\prime}(\mathbf{A})_{0}} \theta_{\varphi}\left(\eta_{1} \otimes \eta_{2}(2)\right)(g) \cdot F^{D^{\prime}}(g) \cdot d g,
$$

with $G^{\prime}(\mathbf{A})_{0}=G^{\prime}(\mathbb{Q}) \cdot G^{\prime}(\mathbf{A})_{+}$, as in $\S 3.8$. In order to analyze the right-hand side of (5.6.1), we need to compute the $K$-types of $\theta_{\varphi}\left(\eta_{1} \otimes \eta_{2}(2)\right)$, as promised in $\S 5.3$. This is again a matter of consulting Kashiwara-Vergne, this time for the $(U(1), U(p, q))$ liftings, with $p+q=2$. We have to suppose $U(1)$ to be the symmetry group of a skew-hermitian form $h$, and to distinguish between signs we say $U(1)=U(1,0)$ (resp. $U(0,1)$ ) if $-i h$ is positive-definite (resp. negative definite), where $i$ is the square root of -1 used to define the additive character $\psi_{\infty}$.

Thus suppose $\chi_{k}\left(e^{i \theta}\right)=e^{i k \theta}, k \in \mathbb{Z}$, is a character of $U(1)$. Let $S \Theta\left(\chi_{k} ; p, q\right)$ denote the restriction of $\Theta\left(U(1,0) \rightarrow U(p, q) ; \chi_{k}\right)$ to $S U(p, q)$. For $k \geq 2$, define the representation $\pi^{\mathbb{H}}(k)$ of $S U(2)$ as in $\S 2.1$, and let $\pi_{k}$ denote the discrete series representation of $S L(2, \mathbb{R})$ with lowest $U(1)$-type $\chi_{k}$. With our choice of additive character, we have the following table:

TABLE 5.6 .2

\begin{tabular}{llll}
$p$ & $q$ & $S \boldsymbol{\Theta}\left(\chi_{k} ; p, q\right)(U(1)=U(1,0))$ & $S \Theta\left(\chi_{k} ; p, q\right)(U(1)=U(0,1))$ \\
\hline 2 & 0 & $\pi^{\mathbb{H}}(k+1)$ if $k>0$, trivial if $k \leq 0$ & $\pi^{\mathbb{H}}(-k+1)$ if $k<0$, trivial if $k \geq 0$ \\
0 & 2 & $\pi^{\mathbb{H}}(-k+1)$ if $k<0$, trivial if $k \geq 0$ & $\pi^{\mathbb{H}}(k+1)$ if $k>0$, trivial if $k \leq 0$ \\
1 & 1 & $\pi_{k+1}$ if $k>0, \pi_{-k-1}$ if $k<0$ & $\pi_{-k+1}$ if $k>0, \pi_{k-1}$ if $k<0$
\end{tabular}

In the last line we have omitted the case $k=0$, which is irrelevant for our purposes, since $k\left(\eta_{i}\right)_{j} \geq 1$ for all $j, i=1,2$. 
Recall that our theta correspondence depends on the choice of $\mathrm{g} \in \mathscr{K}$ with $\operatorname{Tr}_{\mathscr{K} / E}($ я $)=0$. We may assume я satisfies $i \tau(я)<0$ for all $\tau \in \Phi$. Then

$$
U\left(V_{1}\right)\left(E_{j}\right) \cong U(1,0), \quad U\left(V_{2}\right)\left(E_{j}\right) \cong U(0,1) \text { for } j \in \Sigma(D) .
$$

With these conventions, and recalling that $\varphi_{\infty}$ is chosen to be of minimal Howe degree, we find, comparing 5.6.2 with the information in (5.5.12), that $\theta_{\varphi}\left(\eta_{1} \otimes \eta_{2}(2)\right)$ is in the space spanned by functions of the form (5.3.6), with $f_{1}$ holomorphic of weight 2 and $f_{2}$ antiholomorphic of weight $k_{j}+2$ at places in $\Sigma\left(D^{\prime}\right)$. The $K$-types away from $\Sigma\left(D^{\prime}\right)$ can also be determined, but they are less important.

We assume henceforward that $\theta_{\varphi}\left(\eta_{1} \otimes \eta_{2}(2)\right)(g) \neq 0$ for some $\varphi \in \mathscr{S}(\overline{\mathbb{Q}})$; otherwise the rationality criterion in Lemma 5.4.4 is vacuous. Then we may appeal to Lemma 5.3.5, and (5.3.7) and the subsequent results are valid. Applying (5.3.7), we can rewrite the right-hand side of (5.6.1):

$$
\begin{aligned}
& c\left(\varphi_{\infty}, \eta_{1}, \eta_{2}, D^{\prime}\right)^{-1} \cdot L_{\left(\omega, \beta^{-1}\right)}\left(\theta_{\varphi}\left(F^{D^{\prime}}\right)\right) \\
&=\sum_{\alpha} e_{\alpha} \int_{Z_{G^{\prime}}(\mathbf{A}) \cdot G^{\prime}(\mathbb{Q}) \backslash G^{\prime}(\mathbf{A})_{0}}\left[f_{1, \alpha} \otimes f_{2, \alpha} \otimes\left(\bar{f}_{1, \alpha} \otimes \xi_{1}^{-1}\right) \otimes\left(\bar{f}_{2, \alpha} \otimes \xi_{2}^{-1}\right)\right] \\
& \times(g) F^{D^{\prime}}(g) d g .
\end{aligned}
$$

Here $\alpha$ is a finite index set, $e_{\alpha} \in \overline{\mathbb{Q}}$, and for each $\alpha, f_{2, \alpha} \in \pi\left(\eta_{2}, \mathscr{K}\right)^{D^{\prime}}$ (resp. $f_{1, \alpha} \in \pi\left(\eta_{1}, \mathscr{K}\right)^{D^{\prime}}$ ) is an arithmetic automorphic form (resp. the complex conjugate of an arithmetic automorphic form) of type $\left(\underline{k}\left(\eta_{2}\right), 1-w\left(\eta_{2}\right)\right)$ (resp. $\left.\left(\underline{k}\left(\eta_{1}\right), 1-w\left(\eta_{1}\right)\right)\right)$.

Here is what is going on in the classical language. Viewed by restriction as modular forms on the symmetric space $\left(\mathfrak{H}^{ \pm}\right)^{\Sigma\left(D^{\prime}\right)} \times\left(\mathfrak{H}^{ \pm}\right)^{\Sigma\left(D^{\prime}\right)}$ for $\left(D^{\prime} \times\right)^{2}$, $\left[\bar{f}_{1, \alpha} \otimes\left(\bar{f}_{1, \alpha} \otimes \xi_{1}^{-1}\right) \cdot F^{D^{\prime}}\right]$ are all holomorphic in the first copy of $\left(\mathfrak{H}^{ \pm}\right)^{\Sigma\left(D^{\prime}\right)}$ and antiholomorphic in the second copy, of weight $=\left(\right.$ weight of $\left.f\left(\chi^{D^{\prime}}\right)\right)+k\left(\eta_{1}\right)_{j}=$ $k_{j}+2$ at all $j \in \Sigma\left(D^{\prime}\right)$. Similarly, $\left[f_{2, \alpha} \otimes\left(\bar{f}_{2, \alpha} \otimes \xi_{2}^{-1}\right)\right]$, is antiholomorphic in the first copy of $\left(\mathfrak{H}^{ \pm}\right)^{\Sigma\left(D^{\prime}\right)}$ and antiholomorphic in the second copy, again of weight $k\left(\eta_{2}\right)_{j}=k_{j}+2$ at places in $\Sigma\left(D^{\prime}\right)$. Thus the left-hand side of (5.6.1) is a Petersson inner product of $\left[f_{2, \alpha} \otimes\left(\bar{f}_{2, \alpha} \otimes \xi_{2}^{-1}\right)\right]$ against an arithmetic holomorphic modular form of the same weight, where here (and only here!) complex conjugates of arithmetic holomorphic forms are viewed as arithmetic antiholomorphic forms.

Let $\operatorname{pr}_{\eta_{1}}: \mathscr{A}_{0}\left(G^{\prime}\right) \rightarrow \mathscr{A}_{0}\left(G^{\prime}\right)$ denote the orthogonal projection onto the space of forms whose restrictions to $G^{\prime}(\mathbf{A}) \cap\left(D^{\times}\right)^{2}(\mathbf{A})$ lie in $\pi\left(\eta_{2}, \mathscr{K}\right)^{D^{\prime}} \otimes \check{\pi}\left(\eta_{2}, \mathscr{K}\right)^{D^{\prime}}$. By strong multiplicity one, this is the same as the projection onto the space of forms which transform under $\left(D^{\times}\right)^{2}\left(\mathbf{A}^{f}\right)$ via $\pi\left(\eta_{2}, \mathscr{K}\right)^{D^{\prime}} \otimes \check{\pi}\left(\eta_{2}, \mathscr{K}\right)^{D^{\prime}}$. In particular, this projection takes arithmetic automorphic forms to arithmetic automorphic forms. 
Now it is clear that the right-hand side of (5.6.3) equals

$\sum_{\alpha} e_{\alpha} \int_{Z_{G^{\prime}}(\mathbf{A}) \cdot G^{\prime}(\mathbb{Q}) \backslash G^{\prime}(\mathbf{A})_{0}}\left[f_{2, \alpha} \otimes\left(\bar{f}_{2, \alpha} \otimes \xi_{2}^{-1}\right)\right](g) \cdot \operatorname{pr}_{\eta_{1}}\left[f_{1, \alpha} \otimes\left(\bar{f}_{1, \alpha} \otimes \xi_{1}^{-1}\right) \cdot F^{D^{\prime}}\right](g) d g$.

Applying Corollary 2.5.6 and (2.5.4), we find that the right-hand side of (5.6.3) is an algebraic multiple of $q^{D^{\prime}}\left(\eta_{2}, \mathscr{K}\right)^{2}$. We have proved

5.6.4. Lemma. For all $\eta_{1}$ and $\eta_{2}$ satisfying (5.4.6), we have

$$
L_{\left(\omega, \beta^{-1}\right)}\left(\theta_{\varphi}\left(F^{D^{\prime}}\right)\right) \sim_{\overline{\mathbb{Q}}} c\left(\varphi_{\infty}, \eta_{1}, \eta_{2}, D^{\prime}\right) \cdot q^{D^{\prime}}\left(\eta_{2}, \mathscr{K}\right)^{2} .
$$

5.7. Proof of the main theorem. Now we can compute. Comparing Lemmas 5.4.4 and 5.6.4, we have

$$
p_{\mathscr{K}}(\tilde{\beta}, \Psi) \cdot c\left(\varphi_{\infty}, \omega \otimes \pi, D^{\prime}, D\right) \sim_{\overline{\mathbb{Q}}} c\left(\varphi_{\infty}, \eta_{1}, \eta_{2}, D^{\prime}\right) \cdot q^{D^{\prime}}\left(\eta_{2}, \mathscr{K}\right)^{2} ;
$$

recalling (5.4.6), this is equivalent to

$$
p_{\mathscr{K}}\left(\tilde{\eta}_{1} \cdot \tilde{\eta}_{2}^{l}, \Psi\right) \cdot c\left(\varphi_{\infty}, \omega \otimes \pi, D^{\prime}, D\right) \sim_{\overline{\mathbb{Q}}} c\left(\varphi_{\infty}, \eta_{1}, \eta_{2}, D^{\prime}\right) \cdot q^{D^{\prime}}\left(\eta_{2}, \mathscr{K}\right)^{2} .
$$

Comparing Lemma 5.3.8 and Proposition 5.3.9, we have

$$
q^{D^{\prime}}\left(\eta_{1}, \mathscr{K}\right) \cdot q^{D^{\prime}}\left(\eta_{2}, \mathscr{K}\right) \cdot c\left(\varphi_{\infty}, \eta_{1}, \eta_{2}, D^{\prime}\right) \sim_{\overline{\mathbb{Q}}} C_{2} \cdot L_{\mathscr{K}}\left(\tilde{\eta}_{1}, 1\right) \cdot L_{\mathscr{K}}\left(\tilde{\eta}_{2}, 1\right)
$$

Together, (5.7.1) and (5.7.2) yield

$$
\begin{gathered}
q^{D^{\prime}}\left(\eta_{1}, \mathscr{K}\right) \cdot p_{\mathscr{K}}\left(\tilde{\eta}_{1} \cdot \tilde{\eta}_{2}^{l}, \Psi\right) \cdot c\left(\varphi_{\infty}, \omega \otimes \pi, D^{\prime}, D\right) \\
\sim_{\overline{\mathbb{Q}}} C_{2} \cdot L_{\mathscr{K}}\left(\tilde{\eta}_{1}, 1\right) \cdot L_{\mathscr{K}}\left(\tilde{\eta}_{2}, 1\right) q^{D^{\prime}}\left(\eta_{2}, \mathscr{K}\right) .
\end{gathered}
$$

By Lemma 1.6 , we may replace $p_{\mathscr{K}}\left(\tilde{\eta}_{1} \cdot \tilde{\eta}_{2}^{l}, \Psi\right)$ by $p_{\mathscr{K}}\left(\tilde{\eta}_{1}^{l} \cdot \tilde{\eta}_{2}, \imath \Psi\right)$. Applying Shimura's Theorem 2.6.3 (cf. $\S 7.2$, below), Blasius' Theorem 1.8.1, and recalling (5.5.12), we may replace the quadratic periods and $L$-values above with $C M$ periods:

$$
\begin{gathered}
L_{\mathscr{K}}\left(\tilde{\eta}_{1}, 1\right) \sim_{\overline{\mathbb{Q}}}(2 \pi i)^{d} \cdot p_{\mathscr{K}}\left(\tilde{\eta}_{1},\left(\Psi-\Psi^{\prime}\right) \amalg l \Psi^{\prime} \amalg l(\Phi-\Psi)\right), \\
q^{D^{\prime}}\left(\eta_{1}, \mathscr{K}\right) \sim_{\overline{\mathbb{Q}}} p_{\mathscr{K}}\left(\tilde{\eta}_{1}, \imath \Psi^{\prime}\right), \\
L_{\mathscr{K}}\left(\tilde{\eta}_{2}, 1\right) \sim_{\overline{\mathbb{Q}}}(2 \pi i)^{d} \cdot p_{\mathscr{K}}\left(\tilde{\eta}_{2}, l \Phi\right), \quad q^{D^{\prime}}\left(\eta_{2}, \mathscr{K}\right) \sim_{\overline{\mathbb{Q}}} p_{\mathscr{K}}\left(\tilde{\eta}_{2}, l \Psi^{\prime}\right) .
\end{gathered}
$$

If we set $C_{3}=(2 \pi i)^{2 d} \cdot C_{2}$, then $(5.7 .3)$ yields

$$
\begin{aligned}
\left.p_{\mathscr{K}}\left(\tilde{\eta}_{1}, l \Psi^{\prime}\right) \cdot p_{\mathscr{K}}\left(\tilde{\eta}_{1}^{l} \cdot \tilde{\eta}_{2}, \imath \Psi\right) c\left(\varphi_{\infty}, \omega \otimes \pi, D^{\prime}, D\right)\right) \\
\quad \sim_{\overline{\mathbb{Q}}} C_{3} \cdot p_{\mathscr{K}}\left(\tilde{\eta}_{1},\left(\Psi-\Psi^{\prime}\right) \amalg \imath \Psi^{\prime} \amalg l(\Phi-\Psi)\right) \cdot p_{\mathscr{K}}\left(\tilde{\eta}_{2}, l \Phi\right) \cdot p_{\mathscr{K}}\left(\tilde{\eta}_{2}, l \Psi^{\prime}\right) .
\end{aligned}
$$

After several applications of the "monomial relations" (Proposition 1.4(c) and Corollary 1.5$)$, and observing that $\tilde{\eta}_{1} \cdot \tilde{\eta}_{1}^{l}=1,(5.7 .4)$ simplifies as follows:

$$
\begin{aligned}
c\left(\varphi_{\infty}, \omega \otimes \pi, D^{\prime}, D\right) & \sim_{\overline{\mathbb{Q}}} C_{3} \cdot p_{\mathscr{K}}\left(\tilde{\eta}_{1} \cdot \tilde{\eta}_{2}, l \Psi^{\prime} \amalg l(\Phi-\Psi)\right) \\
& \sim_{\overline{\mathbb{Q}}} C_{3} p_{\mathscr{K}}\left(\tilde{\omega}, l \Psi^{\prime} \amalg l(\Phi-\Psi)\right) .
\end{aligned}
$$


Note that the factor of proportionality in (5.1.5) depends only on $\tilde{\omega}$ and not on $\pi$ ! One can find a conceptual justification for its independence of $\beta$ ( $\beta$ depends on the choice of CM point, and one could in principle choose a different CM point), but it can be shown that $c\left(\varphi_{\infty}, \omega \otimes \pi, D^{\prime}, D\right)$ is independent of $\pi$ only if $\Sigma\left(D^{\prime}\right) \subset \Sigma(D)$.

Note further that $C_{3}$, like $C_{2}$, depends only the archimedean data.

Lemma 5.2.3 and Proposition 5.2.4 provide another equation for $c\left(\varphi_{\infty}, \omega \otimes \pi, D^{\prime}, D\right)$ :

$$
q^{D}(\pi) \cdot c\left(\varphi_{\infty}, \omega \otimes \pi, D^{\prime}, D\right) \sim_{\overline{\mathbb{Q}}} C_{1} \cdot L\left(\pi_{\mathscr{K}} \otimes \omega, \frac{1}{2}\right) \cdot q^{D^{\prime}}(\pi) .
$$

Combining (5.7.5) and (5.7.6), we obtain

$$
C_{3} \cdot q^{D}(\pi) \cdot p_{\mathscr{K}}\left(\tilde{\omega}, \imath \Psi^{\prime} \amalg l(\Phi-\Psi)\right) \sim_{\overline{\mathbb{Q}}} C_{1} \cdot L\left(\pi_{\mathscr{K}} \otimes \omega, \frac{1}{2}\right) \cdot q^{D^{\prime}}(\pi),
$$

or

$$
q^{D}(\pi) \cdot p_{\mathscr{K}}\left(\tilde{\omega}, l \Psi^{\prime} \amalg l(\Phi-\Psi)\right) \sim_{\overline{\mathbb{Q}}} C_{4} \cdot L\left(\pi_{\mathscr{K}} \otimes \omega, \frac{1}{2}\right) \cdot q^{D^{\prime}}(\pi)
$$

where $C_{4}$ depends only on the archimedean data. We write

$$
C_{4}=C_{4}\left(\underline{k}, \Sigma(D), \Sigma\left(D^{\prime}\right)\right),
$$

the remaining data $\left(\varphi_{\infty}\right.$ and $\left.\chi^{D}\right)$ presumed subordinate to those indicated.

Now we employ a trick. Replace $D^{\prime}$ by $D(\varnothing)$, in the notation of 2.6.2(b), and replace $D$ by an arbitrary $D^{\prime \prime}$. Let $\Psi^{\prime \prime}=\left\{\tau \in \Phi|\tau|_{E} \in \Sigma\left(D^{\prime \prime}\right)\right\}$. Then $q^{D(\varnothing)}(\pi)=1$ (Lemma 2.5.8), and we obtain another formula for $L\left(\pi_{\mathscr{K}} \otimes \omega, \frac{1}{2}\right)$ :

$$
C_{4}\left(\underline{k}, \Sigma\left(D^{\prime \prime}\right), \varnothing\right) \cdot L\left(\pi_{\mathscr{K}} \otimes \omega, \frac{1}{2}\right) \sim_{\overline{\mathbb{Q}}} q^{D^{\prime \prime}}(\pi) \cdot p_{\mathscr{K}}\left(\tilde{\omega}, l\left(\Phi-\Psi^{\prime \prime}\right)\right) .
$$

This formula is only valid if $\omega$ satisfies the local conditions (i) of Theorem 4.5 for the pair $\left(D(\varnothing), D^{\prime \prime}\right)$. Suppose $\omega$ satisfies these conditions for the pair $\left(D^{\prime}, D\right)$. If $D_{1}$ and $D_{2}$ are quaternion algebras over $E$, let $S\left(D_{1}, D_{2}\right)$ be the set of places $v$ such that $\varepsilon\left(D_{1}\right) \cdot \varepsilon\left(D_{2}\right)=-1$. In order to guarantee that the local conditions remain satisfied for the pair $\left(D(\varnothing), D^{\prime \prime}\right)$, it suffices to assume $S\left(D^{\prime}, D(\varnothing)\right)=S\left(D, D^{\prime \prime}\right)$. In that case, $\Phi-\Psi^{\prime \prime}=\Psi^{\prime} \amalg(\Phi-\Psi)$.

Now assume $D=D(I), D^{\prime}=D\left(I^{\prime}\right)$, and that $I=I^{\prime} \cup\left\{\sigma_{j_{0}}\right\}$, for some $j_{0} \in$ $\{1, \ldots, n\}$. Then $S\left(D, D^{\prime \prime}\right)=S\left(D^{\prime}, D(\varnothing)\right)$ if and only if $D^{\prime \prime}=D\left(\left\{\sigma_{j_{0}}\right\}\right)$. Furthermore, exactly one of each pair $\left(D, D^{\prime}\right)$ and $\left(D^{\prime \prime}, D(\varnothing)\right)$ is ramified at $v_{0}$; the other is unramified at finite places. Thus the hypotheses of Theorem 4.5 are satisfied; in particular, formulas (5.7.7) and (5.7.8) are valid. Combining (5.7.7) and (5.7.8) with the relation $\Phi-\Psi^{\prime \prime}=\Psi^{\prime} \amalg(\Phi-\Psi)$, we obtain

$$
\begin{aligned}
C_{4}(\underline{k}, & \left.\Sigma\left(D^{\prime \prime}\right), \varnothing\right) \cdot q^{D}(\pi) \cdot p_{\mathscr{K}}\left(\tilde{\omega}, l\left(\Phi-\Psi^{\prime \prime}\right)\right) \\
& \sim_{\overline{\mathbb{Q}}} C_{4}\left(\underline{k}, \Sigma(D), \Sigma\left(D^{\prime}\right)\right) \cdot q^{D^{\prime \prime}}(\pi) \cdot p_{\mathscr{K}}\left(\tilde{\omega}, l\left(\Phi-\Psi^{\prime \prime}\right)\right) \cdot q^{D^{\prime}}(\pi),
\end{aligned}
$$

or

$$
q^{D}(\pi) \sim_{\overline{\mathbb{Q}}} C_{5}\left(\underline{k}, \Sigma(D), \Sigma\left(D^{\prime}\right)\right) \cdot q^{D^{\prime \prime}}(\pi) \cdot q^{D^{\prime}}(\pi)
$$


This completes the proof of the first part of Theorem 2.6.5 in the case $|J|=1$. By Corollary 2.6.6, this completes the proof of the main Theorem 2.6.1. Of course, the first part of Theorem 2.6.5 is a consequence of Theorem 2.6.1.

It remains to prove the second part of Theorem 2.6.5. But under the hypotheses of the second part, $D_{i}$ is unramified at all finite primes, $1 \leq i \leq 4$. Thus (by Corollary 4.4.5), with an appropriate choice of $\omega$, the hypotheses of Theorem 4.5 are satisfied for the pairs $\left(D_{1}, D_{2}\right)$ and $\left(D_{3}, D_{4}\right)$; indeed, $S\left(D_{1}, D_{3}\right)=S\left(D_{2}, D_{4}\right)=I$. The argument thus proceeds as above.

5.7.10. Remark. Formula (5.7.8) is a result on special values of $L$-functions, along the lines of those proved by Shimura in [42], but including cases (where $k(\omega)_{j}=1$ for some $j$ ) which Shimura was unable to treat. It will be noticed that, for $D^{\prime}=D(\varnothing)$, Lemma 5.3.7 asserts simply that

$$
c\left(\varphi_{\infty}, \eta_{1}, \eta_{2}, D(\varnothing)\right) \sim_{\overline{\mathbb{Q}}} B_{D(\varnothing)}\left(\theta_{\varphi}\left(\eta_{1} \otimes \eta_{2}(2)\right)\right) .
$$

Thus (5.7.8) can be proved without reference to Shimura's Theorem 2.6.3. In fact, we can compute $C_{4}\left(\underline{k}, \Sigma\left(D^{\prime \prime}\right), \varnothing\right)$, up to algebraic factors, using the relations proved in $\S \S 1.8-9$, and deduce the main results of Shimura's paper [loc.cit.] from (5.7.8). This will be shown in $\S 7$.

\section{RALLIS' INNER PRODUCT FORMULA IN THE UNITARY CASE}

In the monograph [34], Rallis introduced a method for computing the inner product with itself of the theta-lift of an automorphic form from a symplectic group to an orthogonal group. Using an extension of the Weil-Siegel formula, which identifies certain theta functions with Eisenstein series, beyond the range of absolute convergence, the inner product can be identified with the special value of the standard $L$-function of the original symplectic automorphic form, by means of the doubling construction of Piatetski-Shapiro-Rallis.

This program has been completely worked out by Kudla and Rallis [21, 22] for symplectic/orthogonal dual reductive pairs. For general classical groups considered in $\S 3$, including the unitary dual reductive pairs of concern to us here, J-S. Li [26] has established all elements of Rallis' program with the exception of the generalized Weil-Siegel formula; this should appear in forthcoming work of Kudla-Rallis. The purpose of this section is to work out the doubling method for dual reductive pairs over totally real fields of the form $(U(1) \times U(1), U(2) \times$ $U(2))$ and $(U(2), U(2))$. We begin by developing the theory of standard $L$ functions of unitary groups. For future reference, we treat the case of arbitrary dimension.

6.1. Eisenstein series. Let $n$ be a positive integer, and let $S_{n}$ be the $2 n \times$ $2 n$ matrix $\left[\begin{array}{cc}0 & I_{n} \\ I_{n} & 0\end{array}\right]$. Let $G U(n, n) \subset R_{\mathscr{K} / \mathbb{Q}} G L(2 n)_{\mathscr{K}}$ be the group of unitary similitudes of $S_{n}$ :

(6.1.1)

$$
G U(n, n)=\left\{\left.g \in R_{\mathscr{K} / \mathbb{Q}} G L(2 n)_{\mathscr{H}}\right|^{t} g^{l} \cdot S_{n} \cdot g=\nu(g) S_{n}, \nu(g) \in R_{E / \mathbb{Q}} \mathbb{G}_{m, E}\right\} .
$$

Let $U(n, n)=\operatorname{Ker}(\nu)$ be the unitary group. 
The Siegel parabolic of $G U(n, n)$ is the maximal parabolic subgroup

$$
G P=\left\{g=\left[\begin{array}{cc}
A & B \\
0 & D
\end{array}\right]\right\} \subset G U(n, n)
$$

where $A, B$, and $D$ are $n \times n$ matrices over $\mathscr{K} ; P=G P \cap U(n, n)$. The Siegel parabolic is the stabilizer of a maximal isotropic subspace of $\mathscr{K}^{2 n}$. In general, we view these groups as schemes over $\mathbb{Q}$; in particular, $G U(n, n)(\mathbb{Q})$ (resp. $G U(n, n)(\mathbf{A}))$ is the subgroup of $G L(2 n, \mathscr{K})$ (resp. $\left.G L\left(2 n, \mathscr{K}_{\mathbf{A}}\right)\right)$ defined by (6.1.1). However, it is occasionally convenient to view these groups as schemes over $E$, especially when looking at the points over completions of $E$. This should not cause any confusion.

The matrix $g$ in (6.1.2) has the property that $D=\nu(g) \cdot\left({ }^{t} A^{l}\right)^{-1}$. We write $A(g)=A$ for $g \in G P$. For any place $v$ of $E$, let

$$
\delta_{P, v}(g)=|\operatorname{det} \circ \operatorname{Ad}(g)|_{v}^{1 / 2}: G P\left(E_{v}\right) \rightarrow \mathbb{R}_{+}^{\times},
$$

be the modulus character. Then

$$
\delta_{P, v}(g)=\left|N_{\mathscr{K} / E} \circ \operatorname{det}(A(g))\right|_{v}^{n / 2} \cdot|\nu(g)|_{v}^{-n^{2} / 2} .
$$

Let $\delta_{P, \mathbf{A}}(g)=\mid N_{\mathscr{K} / E}$ odet $\left.(A(g))\right|_{\mathbf{A}} ^{n / 2} \cdot|\nu(g)|_{\mathbf{A}}^{-n^{2} / 2}$ be the adelic modulus character, and let

$$
\begin{gathered}
\delta_{P, \mathbf{A}}(g, s)=\left|N_{\mathscr{K} / E} \circ \operatorname{det}(A(g))\right|_{\mathbf{A}}^{n / 2+s} \cdot|\nu(g)|_{\mathbf{A}}^{-n^{2} / 2-n s}, \quad s \in \mathbb{C} ; \\
\delta_{P, \mathbf{A}}^{0}(g)=\left|N_{\mathscr{K} / E} \circ \operatorname{det}(A(g))\right|_{\mathbf{A}} \cdot|\nu(g)|_{\mathbf{A}}^{-n} .
\end{gathered}
$$

Let

$$
\begin{aligned}
I_{s, \mathbf{A}} & =\operatorname{Ind}_{G P(\mathbf{A})}^{G U(\mathbf{A})} \delta_{P, \mathbf{A}}^{0}(g)^{s} \text { (normalized induction) } \\
& =\left\{\mathscr{F}: G U(n, n)(\mathbf{A}) \rightarrow \mathbb{C} \mid \mathscr{F}(p g)=\delta_{P, \mathbf{A}}(p, s) \mathscr{F}(g), p \in G P(\mathbf{A}),\right. \\
& \quad g \in G U(n, n)(\mathbf{A})\} .
\end{aligned}
$$

The local induced representations $I_{s, v}$ and the finite adelic representation $I_{s, \mathbf{A}^{f}}$ are defined analogously.

The group $U(n) \times U(n)$ is embedded in $G U(n, n)(\mathbb{R})$ in the obvious way. Let $K_{\infty}(n, n)=Z_{G U(n, n)}(\mathbb{R}) \cdot[U(n) \times U(n)] \subset G U(n, n)(\mathbb{R})$, and let $K_{\infty}$ be any subgroup of $G U(n, n)(\mathbb{R})$ which is conjugate to $K_{\infty}(n, n)$. The choice of $K_{\infty}$ will be dictated by the circumstances. The lattice $\left(\mathscr{O}_{\mathscr{K}}\right)^{2 n} \subset \mathscr{K}^{2 n}$ is self-dual with respect to the form $S_{n}$. Let $K_{f, n} \subset G U_{\mathscr{K}}\left(n, n, \mathbf{A}^{f}\right)$ be the stabilizer of the corresponding adelic lattice. Then $K_{\infty} \cdot K_{f}$ satisfies the Iwasawa decomposition $U_{\mathscr{H}}(s, \mathbf{A})=P(\mathbf{A}) \cdot K_{\infty} \cdot K_{f}$. Define

$$
\begin{gathered}
\delta_{n}(g)=\left\|t^{-n} N_{\mathscr{K} / E} \operatorname{det}(A)\right\|_{\mathbf{A}}, \\
g=\left(\begin{array}{cc}
A & B \\
0 & t\left(A^{l}\right)^{-1}
\end{array}\right) k \in G U_{\mathscr{H}}(n, n, \mathbf{A}), k \in K_{\infty} \cdot K_{f} .
\end{gathered}
$$


A section $\mathscr{F}(g, s)$ is a function which, to each $s \in \mathbb{C}$, associates a function $\mathscr{F}(g, s) \in I_{s, \mathbf{A}}$ satisfying the following continuity property: $\delta_{n}(g)^{-s} \cdot \mathscr{F}(g, s)$ is a continuous $K_{\infty}$-finite function of $g$, holomorphic in $s$.

For $\operatorname{Re}(s)$ sufficiently large, the Eisenstein series

$$
E(g, s, \mathscr{F})=\sum_{\gamma \in G P(\mathbb{Q}) \backslash G U(n, n)(\mathbb{Q})} \mathscr{F}(\gamma g, s)
$$

converge absolutely to an automorphic form on $G U(n, n)$, and extends meromorphically to a function on $\mathbb{C}$, with no pole on the imaginary axis.

6.2. The doubling method for unitary groups. Let $V$ be an $n$-dimensional vector space over $\mathscr{K}$ with hermitian inner product $\langle\cdot, \cdot\rangle_{V}$, relative to $E$; let $U(V)$ and $G U(V)$ be the corresponding unitary group and group of unitary similitudes. Define $-V$ as in 3.5.3. Then the hermitian space $V^{(2)}=V \oplus(-V)$ contains a maximal isotropic subspace $V^{\Delta}=\{(v, v), v \in V\}$ of dimension $n$. Thus $G U\left(V^{(2)}\right) \cong G U(n, n)$ as algebraic groups over $\mathbb{Q}$. Fixing such an isomorphism, it follows that the group

$$
G(U(-V) \times U(V))=\left\{\left(g, g^{\prime}\right) \in G U(-V) \times G U(V) \mid \nu(g)=\nu\left(g^{\prime}\right)\right\}
$$

embeds in $G U(n, n)$ through its natural diagonal action.

In this section, we write $G=G(U(-V) \times U(V)), Z$ the identity component of its center, and let $\left(g, g^{\prime}\right)$ be a typical element of $G$. Projection on the two factors identifies $Z \cong Z_{G U(V)} \cong Z_{G U(-V)}$. Let $Z_{0} \subset Z$ denote the subgroup fixed by conjugation of $\mathscr{K}$ over $E$. Choose a Haar measure $d g$ on $G(\mathbf{A})$; we assume it is of the form $\Pi d g_{v}$ where, for almost all places $v$ of $E, d g_{v}$ takes volume 1 on a fixed hyperspecial maximal compact subgroup $K_{v} \subset G U\left(E_{v}\right)$. Let $\left(\pi, H_{\pi}\right)$ be an irreducible cuspidal automorphic representation of $G U(V) \cong G U(-V)$ with central character $\kappa_{\pi}: Z(\mathbf{A}) / Z(\mathbb{Q}) \rightarrow \mathbb{C}^{\times} ;$let $\xi_{\pi}$ denote the restriction of $\kappa_{\pi}$ to $Z_{0}(\mathbf{A}) / Z_{0}(\mathbb{Q})$. Let $\check{\pi}$ be the contragredient to $\pi$.

The standard L-function of $\pi$ is an Euler product attached to the restriction of $\pi$ to $U(V)$. Fix an irreducible automorphic representation $\pi_{U}$ of $U(V)$ in this restriction. There is a finite set $S$ of places of $E$ such that, for $v \notin S$, the local component $\pi_{U, v}$ is unramified and is generated over $U\left(V, E_{v}\right)$ by a $K_{v}$-fixed vector. Let $\Phi_{n}$ be the $n \times n$ matrix with $i j$-entry $(-1)^{i-1} \delta_{i, n-j+1}$. Now the $L$-group of $U(V)$ over $E$ has a quotient of the form $G L(n, \mathbb{C}) \rtimes$ $\operatorname{Gal}(\mathscr{K} / E)$, where $l \in \operatorname{Gal}(\mathscr{K} / E)$ acts on $G L(n, \mathbb{C})$ by $g \mapsto \Phi_{n} t_{g}^{-1} \Phi_{n}^{-1}$. This group has a standard $2 n$-dimensional representation $\mathrm{St}$, induced from the identity map $G L(n, \mathbb{C}) \rightarrow G L(n, \mathbb{C})$. Let $L\left(\pi_{v}, s, \mathrm{St}\right)$ be the Langlands Euler factor for $v \in S$, corresponding to the unramified representation $\pi_{U, v}$ and St, and let $L^{S}(\pi, s, \mathrm{St})$ be the partial Euler product $\prod_{v \notin S} L\left(\pi_{v}, s, \mathrm{St}\right)$.

Choose isomorphisms of $G(\mathbf{A})$-modules:

$$
\pi \stackrel{\sim}{\rightarrow} \bigotimes \pi_{v}, \quad \check{\pi} \stackrel{\sim}{\rightarrow} \bigotimes \check{\pi}_{v}
$$


where $\pi_{v}$ is an admissible irreducible representation of $G U(V)_{v}$; the product is taken over primes of $\mathbb{Q}$. If $f \in H_{\pi}, f^{\prime} \in H_{\check{\pi}}$, and $\mathscr{F}(g, s)$ is a section, the Piatetski-Shapiro-Rallis zeta integral:

$$
Z\left(s, f, f^{\prime}, \mathscr{F}\right)=\int_{Z(\mathbf{A}) \cdot G(\mathbb{Q}) \backslash G(\mathbf{A})} E\left(i_{V}\left(g, g^{\prime}\right), s, \mathscr{F}\right) f(g) f^{\prime}\left(g^{\prime}\right) d g d g^{\prime}
$$

is well defined. Suppose $\mathscr{F}, f$, and $f^{\prime}$ are factorizable, relative to (6.2.1), as elements of $\otimes I_{s, v}, \otimes \pi_{v}$, and $\otimes \check{\pi}_{v}$, respectively:

$$
\mathscr{F}=\bigotimes \mathscr{F}_{v}, \quad f=\bigotimes f_{v}, \quad f^{\prime}=\bigotimes f_{v}^{\prime}
$$

In particular, for almost all primes $v, \pi_{v}$ is unramified and $f_{v}$ and $f_{v}^{\prime}$ are normalized spherical vectors, so that the local pairing $\left(f_{v}, f_{v}^{\prime}\right)=1$. Then the basic identity of [6, Part A], worked out in this case by $\mathrm{Li}$ [26], takes the following form:

$$
d_{n}(s) Z\left(s, f, f^{\prime}, \mathscr{F}\right)=\left\langle f, f^{\prime}\right\rangle_{V} \cdot \prod_{v \in S} Z_{v}\left(s, f, f^{\prime}, \mathscr{F}\right) \cdot L^{S}\left(\pi, s+\frac{1}{2}, \mathrm{St}\right)
$$

Here $S$ is a finite set of places, consisting of $\infty$ and every place at which $V$ is not quasi-split, $\pi$ is not an unramified representation, or $f, f^{\prime}$, and $\mathscr{F}$ are not standard spherical vectors. The local factors $Z_{v}\left(s, f, f^{\prime}, \mathscr{F}\right)$ are defined by

$$
Z_{v}\left(s, f, f^{\prime}, \mathscr{F}\right)=\int_{U_{v}} \mathscr{F}_{v}\left(i_{V}\left(h_{v}, 1\right), s\right) c_{f, f^{\prime}, v}\left(h_{v}\right) d h_{v}
$$

where $U_{v}$ is the local unitary group and

$$
c_{f, f^{\prime}, v}\left(h_{v}\right)=\left(f_{v}, f_{v}^{\prime}\right)^{-1} \cdot\left(\pi_{v}\left(h_{v}\right) f_{v}, f_{v}^{\prime}\right)
$$

is the matrix coefficient, normalized to take value 1 at 1 . The global period $\left\langle f, f^{\prime}\right\rangle_{V}$ is a Petersson inner product:

$$
\left\langle f, f^{\prime}\right\rangle_{V}=\int_{Z(\mathbf{A}) \cdot G U(V, \mathbb{Q}) \backslash G U(V, \mathbf{A})} f(g) f^{\prime}(g) d g .
$$

Finally, the normalizing factor $d_{n}(s)$ is the following product of partial $L$ functions:

$$
d_{n}(s)=\prod_{0 \leq j<n / 2} \zeta_{E}^{S}(2 s+n-2 j) \prod_{0<j \leq n / 2} L^{S}\left(2 s+n-2 j+1, \varepsilon_{\mathscr{K}}\right),
$$

where $\zeta_{E}^{S}$ (resp. $L^{S}\left(*, \varepsilon_{\mathscr{K}}\right)$ ) is the Dedekind zeta function (resp. the Dirichlet $L$-function attached to the quadratic character $\varepsilon_{\mathscr{K}}$ ) with the factors at $S$ removed. For any place $v$ of $E$, let $d_{n, v}(s)$ be the corresponding product of local Euler factors; here we allow $v \in S$.

This formulation requires some justification. Both [6] and [26] consider unitary groups rather than unitary similitude groups. The generalization to similitude groups is simple. As in [6, Part A, $\S 1]$, the zeta integral (6.2.2) breaks up as a sum over the finite set

$$
G P(\mathbb{Q}) \backslash G U(n, n)(\mathbb{Q}) / G U(V,-V)(\mathbb{Q})
$$


of integrals, all of which vanish except that corresponding to the identity coset. Let $I\left(\gamma_{0}\right)$ be the integral corresponding to the identity coset:

$$
I\left(\gamma_{0}\right)=\int_{Z(\mathbf{A}) \cdot G U(V)(\mathbb{Q}) \backslash G U(V,-V)(\mathbf{A})} \mathscr{F}\left(i_{V}\left(g, g^{\prime}\right), s\right) f(g) f^{\prime}\left(g^{\prime}\right) d g d g^{\prime},
$$

where $G U(V)(\mathbb{Q})$ is embedded diagonally in $G U(V,-V)(\mathbf{A})$. Then $I\left(\gamma_{0}\right)=\int_{Z(\mathbf{A}) \cdot G U(V)(\mathbb{Q}) \backslash G U(V,-V)(\mathbf{A})} \mathscr{F}\left(i_{V}\left[\left(g^{\prime}, g^{\prime}\right) \cdot\left(g^{\prime},-1 \cdot g, 1\right)\right], s\right) f(g) f^{\prime}\left(g^{\prime}\right) d g d g^{\prime}$.

Now $\left(g, g^{\prime}\right) \in G U(V,-V)(\mathbf{A})$ implies that $h=g^{\prime,-1} \cdot g \in U(V)(\mathbf{A})$. Furthermore, $i_{V}\left(g^{\prime}, g^{\prime}\right) \in G P(\mathbf{A})$, with $A\left(i_{V}\left(g^{\prime}, g^{\prime}\right)\right)=g^{\prime}, \nu\left(i_{V}\left(g^{\prime}, g^{\prime}\right)\right)=\nu\left(g^{\prime}\right)$. Thus

$$
\begin{aligned}
\mathscr{F}\left(i_{V}\left[\left(g^{\prime}, g^{\prime}\right) \cdot(h, 1)\right], s\right) & =\delta_{P, \mathbf{A}}\left(i_{V}\left(g^{\prime}, g^{\prime}\right), s\right) \cdot \mathscr{F}\left(i_{V}(h, 1), s\right) \\
& =\mathscr{F}\left(i_{V}(h, 1), s\right) .
\end{aligned}
$$

Hence $I\left(\gamma_{0}\right)$ simplifies to

$$
\begin{aligned}
\int_{U(V, \mathbf{A})} \mathscr{F}\left(i_{V}(h, 1), s\right)\left[\int_{Z(\mathbf{A}) \cdot G U(V, \mathbb{Q}) \backslash G U(V, \mathbf{A})} f\left(g^{\prime} h\right) f^{\prime}\left(g^{\prime}\right) d g^{\prime}\right] d h \\
=\left\langle f, f^{\prime}\right\rangle \cdot \int_{U(V, \mathbf{A})} \mathscr{F}\left(i_{V}(h, 1), s\right) \cdot \prod_{v}\left(f_{v}, f_{v}^{\prime}\right)^{-1} \cdot\left(\pi_{v}\left(h_{v}\right) f_{v}, f_{v}^{\prime}\right) d h
\end{aligned}
$$

by uniqueness of the local pairings $\pi_{v} \otimes \check{\pi}_{v} \rightarrow \mathbb{C}$. The basic identity now follows from the local computations of $\mathrm{Li}$.

Let $Z_{f}\left(s, f, f^{\prime}, \mathscr{F}\right)=\prod_{v \in S_{f}} Z_{v}\left(s, f, f^{\prime}, \mathscr{F}\right)$ be the product of the bad nonarchimedean factors. For any $\sigma \in \operatorname{Aut}(\mathbb{C})$, we define $Z_{f}\left(s, f^{\sigma}, f^{\prime, \sigma}, \mathscr{F}^{\sigma}\right)$ $=\prod_{v \in S_{f}} Z_{v}\left(s, f^{\sigma}, f^{\prime, \sigma}, \mathscr{F}^{\sigma}\right)$ as follows. There is a $\sigma$-linear intertwining map $\pi_{v} \otimes \check{\pi}_{v} \rightarrow \pi_{v}^{\sigma} \otimes \check{\pi}_{v}^{\sigma}$ which takes the local pairing $B_{v}: \pi_{v} \otimes \check{\pi}_{v} \rightarrow \mathbb{C}$ to the local pairing $B_{v}^{\sigma}: \pi_{v}^{\sigma} \otimes \check{\pi}_{v}^{\sigma} \rightarrow \sigma(\mathbb{C})=\mathbb{C}$; this takes $f_{v} \otimes f_{v}^{\prime}$ to $\left(f_{v} \otimes f_{v}^{\prime}\right)^{\sigma}$. We define the local factor $\mathscr{F}_{v}{ }^{\sigma}$ by letting Aut $(\mathbb{C})$ act on sections of $I_{s, v}$ by acting on their restrictions to a chosen special maximal compact subgroup $K_{v} \subset G U_{v}$. Then

$$
Z_{v}\left(s, f^{\sigma}, f^{\prime, \sigma}, \mathscr{F}^{\sigma}\right)=\int_{U_{v}} \mathscr{F}_{v}^{\sigma}\left(i_{V}\left(h_{v}, 1\right), s\right) \mathcal{c}_{\left(f \otimes f^{\prime}\right)^{\sigma}, v}\left(h_{v}\right) d h_{v},
$$

where

$c_{\left(f \otimes f^{\prime}\right)^{\sigma}, v}\left(h_{v}\right)=\left[B_{v}^{\sigma}\left(f_{v} \otimes f_{v}^{\prime}\right)^{\sigma}\right]^{-1} \cdot B_{v}^{\sigma}\left(\pi_{v}^{\sigma} \otimes \check{\pi}_{v}^{\sigma}\left(h_{v}, 1\right)\left(f_{v} \otimes f_{v}^{\prime}\right)^{\sigma}\right)=c_{f, f^{\prime}, v}\left(h_{v}\right)^{\sigma}$.

6.2.7. Lemma. Let $m_{0} \in \mathbb{Q}$ satisfy $m_{0} \equiv n / 2(\bmod \mathbb{Z})$.

(i) For all $\sigma \in \operatorname{Aut}(\mathbb{C}), Z_{f}\left(m_{0}, f, f^{\prime}, \mathscr{F}\right)^{\sigma} \sim Z_{f}\left(m_{0}, f^{\sigma}, f^{\prime}, \sigma, \mathscr{F}^{\sigma}\right)$.

(ii) Furthermore, if $\pi_{f}$ is defined over a field $L, f_{v} \otimes f_{v}^{\prime}$ belongs to an $L$ rational model of $\pi_{v} \otimes \check{\pi}_{v}$ for all $v \in S_{f}$, and $\left.\mathscr{F}\right|_{K_{v}}$ takes values in $L$. Then $Z_{f}\left(m_{0}, f, f^{\prime}, \mathscr{F}\right) \in L$.

Proof. This is proved in the same way as Lemma 4.9 .9 of [5]. We review the argument. It is known [32, especially Theorem 2.3] that the local factor 
$Z_{v}\left(s, f, f^{\prime}, \mathscr{F}\right)$ is a rational function of $N v^{-s}$. The measure has been normalized to take rational values, and $\delta_{P, v}\left(g, m_{0}\right)$ takes values in $\mathbb{Q}$ for $m_{0}$ as in the statement of the lemma. It thus follows that $\operatorname{Aut}(\mathbf{C})$ acts on $Z_{v}$ through its action on the integrand:

$(6.2 .7 .1)$

$$
Z_{v}\left(m_{0}, f, f^{\prime}, \mathscr{F}\right)^{\sigma}=\int_{U_{v}} \mathscr{F}_{v}^{\sigma}\left(i_{V}\left(h_{v}, 1\right) m_{0}\right) c_{f, f^{\prime}, v}\left(h_{v}\right)^{\sigma} d h_{v}, \quad \sigma \in \operatorname{Aut}(\mathbb{C}) .
$$

Here we understand that the right-hand side is extended to all $s$ by analytic continuation and then evaluated at $s=m_{0}$. Part (i) of the lemma now follows from the definitions.

Now suppose the hypotheses of (ii) are satisfied. We have to show that

$$
c_{f, f^{\prime}, v}\left(h_{v}\right)^{\sigma}=c_{f, f^{\prime}, v}\left(h_{v}\right) \text { for all } \sigma \in \operatorname{Aut}(\mathbb{C} / L) .
$$

But $\pi\left(h_{v}\right) f_{v} \otimes f_{v}^{\prime} \in \pi_{v} \otimes \check{\pi}_{v}(L)$ for all $h_{v} \in U_{v}$, and by Schur's lemma there is a constant $b$ such that $b^{-1} B_{v}\left(\pi_{v} \otimes \check{\pi}_{v}(L)\right) \subset L$. Thus (6.2.7.2) follows from the definition of $c_{f, f^{\prime}, v}$.

Henceforward, we only consider the cases $n \leq 2$. When $n=1, V=\mathscr{K}$, and $\pi$ is a Hecke character $\eta$. Then (cf. $[7,1.2]$ )

$$
L(\eta, s, \mathrm{St})=L_{\mathscr{K}}(\tilde{\eta}, s), \quad \tilde{\eta}=\eta / \eta^{l} .
$$

Here $\tilde{\eta}$ should be viewed as the base change from $U(\mathscr{K})_{E}$ to $G L(1)_{\mathscr{K}}$ of the restriction of $\eta$ to $U(\mathscr{K})_{E}$. Note that $L_{\mathscr{K}}(\tilde{\eta}, s)=L_{\mathscr{K}}\left(\tilde{\eta}^{-1}, s\right)=L_{\mathscr{K}}\left(\tilde{\eta}^{l}, s\right)$.

When $n=2$, we may assume $V=D, G U(V)=G U_{\mathscr{K}}(D)$, for some quaternion algebra $D$ over $E$ which splits over $\mathscr{K}$. Let $\Pi=\omega \otimes \pi^{D}$ be an automorphic representation of $G U_{\mathscr{K}}(D)$, with $\omega$ a Hecke character of $\mathscr{K}, \pi^{D}$ an automorphic representation of $D^{\times}$associated to the automorphic representation $\pi$ of $G L(2)_{E}$, such that $\xi_{\pi} \cdot \xi_{\omega}=1$.

6.2.9. Lemma. Let $\pi_{\mathscr{K}}$ denote the base change of $\pi$ to $\mathscr{K}$. Then $L^{S}(\Pi, s, \mathrm{St})$ $=L_{\mathscr{K}}^{S}\left(\pi_{\mathscr{K}} \otimes \omega, s\right)$ is the partial Hecke-Jacquet-Langlands $L$-function for $G L(2)_{\mathscr{K}}$ (in the unitary normalization) attached to the twist of $\pi_{\mathscr{K}}$ by $\omega$.

Proof. This is a simple computation. We consider only places $v$ at which $D$ is split. Write $G U_{v}=G U(D)\left(E_{v}\right), U_{v}=U(D)\left(E_{v}\right)$. We may identify

$$
G U_{v}=\left\{\left.g \in G L\left(2, \mathscr{K}_{v}\right)\right|^{t} g^{-1}=\nu(g) g^{l}, \nu(g) \in E_{v}^{\times}\right\},
$$

and the map $\mathscr{K}_{v}^{\times} \times D_{v}^{\times} \rightarrow G U_{v}$ is given by $(k, d) \mapsto\left(k \cdot I_{2}\right) d$, where $I_{2}$ is the identity matrix.

Suppose first that $v$ is a place of $E$ that splits as $w \cdot w^{\prime}$ in $\mathscr{K}$. Then (6.2.10) identifies

$$
U_{v} \cong\left\{\left(g,{ }^{t} g^{-1}\right) \in G L\left(2, E_{v}\right)^{2} \cong G L\left(2, \mathscr{K}_{w}\right) \times G L\left(2, \mathscr{K}_{w^{\prime}}\right)\right\} .
$$

Sending $\left(g,{ }^{t} g^{-1}\right) \mapsto g$ defines an isomorphism $U_{v} \stackrel{\sim}{\rightarrow} G L\left(2, E_{v}\right)$. We use this isomorphism to transfer $\Pi_{U, v}$ to an admissible representation $\Pi_{G L, v}$ 
of $G L\left(2, E_{v}\right)$. Then the local Euler factor $L\left(\Pi_{v}, s, \mathrm{St}\right)=L\left(\Pi_{G L, v}, s\right)$. $L\left(\check{\Pi}_{G L, v}, s\right)$, where $\check{\Pi}_{G L, v}$ is the contragredient of $\Pi_{G L, v}$. But in terms of our given map $\mathscr{K}_{v}^{\times} \times D_{v}^{\times} \rightarrow G U_{v}$, it is easy to see, using the formula $\xi_{\pi} \cdot \xi_{\omega}=1$, that

$$
L\left(\pi_{\mathscr{K}, w} \otimes \omega_{w}, s\right)=L\left(\Pi_{G L, v}, s\right), \quad L\left(\pi_{\mathscr{K}, w^{\prime}} \otimes \omega_{w^{\prime}}, s\right)=L\left(\check{\Pi}_{G L, v}, s\right) .
$$

The case of nonsplit $v$ is analogous.

6.3. The $U\left(D^{\prime}\right) \times U(D)$ lift. We now return to the situation of $\S 5$. Proposition 5.2.4 and Theorem 4.5 are proved simultaneously, by applying the Rallis doubling method.

Let $D$ and $D^{\prime}$ be quaternion division algebras over $E$, with $\Sigma(D) \subset \Sigma\left(D^{\prime}\right)$. Consider the seesaw diagram (3.5.3.1) when $V=D^{\prime}, V^{\prime}=D$; this is the case discussed in Lemma 3.3. Thus $G U_{\mathscr{K}}\left(D^{\prime} \oplus\left(-D^{\prime}\right)\right) \cong G U_{\mathscr{K}}(2,2)$, viewed as the similitude group of the hermitian form $S_{2}$. We have

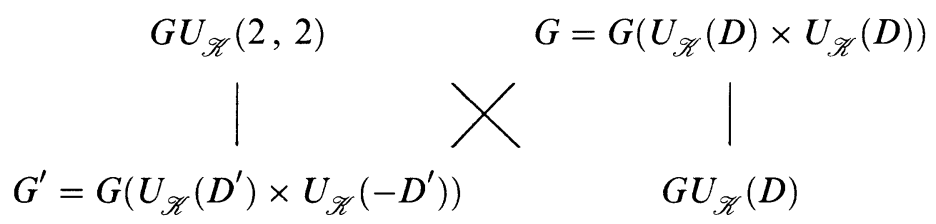

The action $r_{\psi}$ of $R\left(D, D^{\prime} \oplus\left(-D^{\prime}\right)\right)$ on the Schwartz-Bruhat space $\mathscr{S}\left(D(\mathbf{A})^{2}\right)$ was described in the proof of Lemma 3.3. Suppose $\varphi \in \mathscr{S}\left(D(\mathbf{A})^{2}\right)$, and let $\mathscr{F}_{\varphi}(g)=r_{\psi}(g, \alpha) \varphi(0)$ for $g \in G U_{\mathscr{H}}(2,2, \mathbf{A})$ and $\alpha \in G U_{\mathscr{H}}(D)$ such that $\nu(g)=\nu^{\prime}(\alpha)$, where $\nu$ and $\nu^{\prime}$ are the respective similitude factors. (Such $\alpha$ exists only when the sign of $\nu(g)$ is positive at every real prime in $\Sigma-\Sigma\left(D^{\prime}\right)$; otherwise no condition is imposed). Let $P$ be the subgroup of $G_{\mathscr{H}}(2,2)$ of elements of the form

$$
\left(\begin{array}{cc}
A & B \\
0 & t\left(A^{l}\right)^{-1}
\end{array}\right)
$$

with $A \in G L(2, \mathscr{K}), B$ hermitian in $M(2, \mathscr{K})$, and $t \in E^{\times}$. Then (cf. $(3.4 .1-2))$ :

$$
\mathscr{F}_{\varphi}(p \cdot g)=\left\|t^{-2} N_{\mathscr{K} / E} \operatorname{det}(A)\right\|_{\mathbf{A}} \cdot \mathscr{F}_{\varphi}(g), \quad p=\left(\begin{array}{cc}
A & B \\
0 & t\left(A^{l}\right)^{-1}
\end{array}\right) \in P(\mathbf{A}) .
$$

Thus $\mathscr{F}_{\varphi} \in I_{0, \mathbf{A}}$, in the notation of (6.1.5), with $n=2$. Let $\mathscr{F}_{\varphi}(g, s)=\delta_{2}(g)^{s}$. $\mathscr{F}_{\varphi}(g)$ for $g \in G U_{\mathscr{K}}(2,2, \mathbf{A})$, in the notation of $\S 6.1$. Then $\mathscr{F}_{\varphi}(g, s)$ is a section, in the sense of $\S 6.1$, and we can define the Eisenstein series $E\left(g, s, \mathscr{F}_{\varphi}\right)$. It is holomorphic on the imaginary axis, in particular at $s=0$. In this case, the Weil-Siegel formula in the case of unitary groups would yield the following identity:

6.3.3. For all $\varphi \in \mathscr{S}\left(D(\mathbf{A})^{2}\right)$,

$$
\theta_{\varphi}\left(G U_{\mathscr{K}}(D) \rightarrow G U_{\mathscr{K}}(2,2) ; 1\right)=2 \cdot E\left(g, 0, \mathscr{F}_{\varphi}\right),
$$

where 1 is the constant function. 
In the case of the symplectic group the analogous result is a special case of a theorem of Kudla and Rallis [22, 24]. Their methods should carry over with little change to the unitary case, and we ask the reader's indulgence in admitting 6.3.3 until its proof appears. We have used the hypothesis that $D$ be a division algebra to guarantee that the integral defining the left-hand side of the formula is absolutely convergent (alternatively, that the constant function 1 is a cusp form). The theorem should remain true (cf. [24]) if the definition of the theta lift is modified, but the more general case will not be necessary.

6.3.4. Proofs of Theorem 4.5 and Proposition 5.2.4. We apply the seesaw reciprocity formula 3.8 .4 to the diagram $(6.3 .1)$ to express $B_{D}\left(\theta_{\varphi}\left(F^{D^{\prime}}\right)\right)$ as a zeta integral (6.2.2), with $s=0$. In this case, $\mathscr{G} \mathscr{U}^{\prime}(\mathbf{A})_{0}=\mathscr{G}^{\prime}(\mathbf{A})=G U_{\mathscr{K}}(D(\mathbf{A}))$, $\mathscr{G V}(\mathbf{A})_{0}=\mathscr{G}(\mathbf{A})=G\left(U_{\mathscr{H}}\left(D^{\prime}(\mathbf{A})\right)^{\times} U_{\mathscr{K}}\left(-D^{\prime}(\mathbf{A})\right)\right)$. We have $F_{\mathscr{U}^{\prime}}=1, F_{\mathscr{V}}=$ $F^{D^{\prime}}$. Then

$$
B_{D}\left(\theta_{\varphi}\left(F^{D^{\prime}}\right)\right)=\left\langle\theta_{\varphi}\left(F_{\mathscr{V}}\right), F_{\mathscr{U}^{\prime}}\right\rangle_{\mathscr{U}^{\prime}}=\left\langle\theta_{\varphi}\left(F_{\mathscr{U}^{\prime}}\right), F_{\mathscr{V}}\right\rangle_{\mathscr{V}}=2\left\langle E\left(g, 0, \mathscr{F}_{\varphi}\right), F^{D^{\prime}}\right\rangle_{\mathscr{V}} \cdot
$$

We have to show that

For an appropriate choice of $\varphi$ and $F^{D^{\prime}},\left\langle E\left(g, 0, \mathscr{F}_{\varphi}\right), F^{D^{\prime}}\right\rangle_{\mathscr{V}}$ $\neq 0$

There is a constant $C_{1}=C_{1}\left(\varphi_{\infty}, \underline{k}, \chi, D^{\prime}, D\right) \in \mathbb{C}^{\times}$such that, for all $\varphi \in \mathscr{S}(\overline{\mathbb{Q}})$,

$$
\begin{aligned}
B_{D}\left(\theta_{\varphi}\left(F^{D^{\prime}}\right)\right) & =2\left\langle E\left(g, 0, \mathscr{F}_{\varphi}\right), F^{D^{\prime}}\right\rangle_{\mathscr{V}} \\
& \sim_{\overline{\mathbb{Q}}} C_{1} \cdot L\left(\pi_{\mathscr{K}} \otimes \omega, \frac{1}{2}\right) \cdot q^{D^{\prime}}(\pi) .
\end{aligned}
$$

Now $\left\langle E\left(g, 0, \mathscr{F}_{\varphi}\right), F^{D^{\prime}}\right\rangle_{\mathscr{V}}=\left.Z\left(s, f, f^{\prime}, \mathscr{F}_{\varphi}\right)\right|_{s=0}$, with $f=\omega \otimes f\left(\chi^{D^{\prime}}\right)$, $f^{\prime}=\omega^{-1} \otimes \check{f}\left(\chi^{D^{\prime}}\right)$. Thus the basis identity (6.2.3) yields

$$
\begin{aligned}
d_{2}(0) & \left\langle E\left(g, 0, \mathscr{F}_{\varphi}\right), F^{D^{\prime}}\right\rangle_{\mathscr{V}} \\
= & \left\langle f\left(\chi^{D^{\prime}}\right), \check{f}\left(\chi^{D^{\prime}}\right)\right\rangle_{D^{\prime}} \cdot L^{S}\left(\pi_{\mathscr{K}} \otimes \omega, \frac{1}{2}, \mathrm{St}\right) \cdot\left[\prod_{v \in S} Z_{v}\left(s, f, f^{\prime}, \mathscr{F}_{\varphi}\right)\right]_{s=0} ;
\end{aligned}
$$

here we have used Lemma 6.2.9. We remark that the notation $\langle\cdot, \cdot\rangle_{D^{\prime}}$ has been defined twice, in $\S 2.5$ as an integral over $\mathscr{M}_{D}$, and in (6.2.5), as an integral (effectively) over $\mathscr{M}_{\mathscr{K}, D}$. By (1.2.3) and the normalization of measures in the Notation section, these two spaces have the same volume up to rational multiples; here the factor $(2 \pi i)^{-|\Sigma(D)|}$ in $(2.5 .2)$ is essential (cf. the discussion of rational measures in the introduction to [13]). Thus up to a rational factor the two definitions coincide. Now

$$
d_{2}(0)=\zeta_{E}^{S}(2) \cdot L^{S}\left(1, \varepsilon_{\mathscr{K}}\right)=d_{1} \cdot G\left(\varepsilon_{\mathscr{K}}\right) \cdot(2 \pi i)^{3 d}=d_{2}
$$


for some $d_{1} \in \mathbb{Q}^{\times}$. Next, with our choice of $f\left(\chi^{D^{\prime}}\right)$, we have

$$
0 \neq\left\langle f\left(\chi^{D^{\prime}}\right), \check{f}\left(\chi^{D^{\prime}}\right)\right\rangle_{D^{\prime}} \sim_{\overline{\mathbb{Q}}} q^{D^{\prime}}(\pi) .
$$

We have chosen $\omega$ so that $L\left(\pi_{\mathscr{K}} \otimes \omega, \frac{1}{2}, \mathrm{St}\right) \neq 0$; therefore the partial $L$ function is also not equal to zero, since (by the classification of unitary representations of $G L(2)$ over a local field) the local factors have no poles for $\operatorname{Re}(s) \geq \frac{1}{2}$.

It remains to be shown that

$$
\begin{aligned}
& \text { The data } f, f^{\prime} \text {, and } \mathscr{F}_{\varphi} \text { can be chosen so that } Z_{v}\left(s, f, f^{\prime}, \mathscr{F}_{\varphi}\right) \\
& \neq 0 \text { at } s=0 \text {. }
\end{aligned}
$$

This will be proved in greater generality in forthcoming joint work with Kudla, so we only sketch an argument that works in the present case. First assume $D^{\prime}$ ramified at $v$. Then it is easy to see that $(6.3 .10)$ is equivalent to

$$
\operatorname{Hom}_{G^{\prime}\left(E_{v}\right)_{+}}\left(\left[\pi^{D} \otimes \omega\right](2) \otimes\left(\Theta\left(G U_{\mathscr{K}}\left(D_{v}\right) \rightarrow G U(2,2)_{v} ; 1_{G\left(E_{v}\right)}\right), \mathbb{C}\right) \neq 0 .\right.
$$

Indeed, the zeta integral $Z_{v}\left(s, f, f^{\prime}, \mathscr{F}_{\varphi}\right)$ defines a pairing in the space in (6.3.11), so (6.3.10) implies (6.3.11). On the other hand, $G^{\prime}\left(E_{v}\right)$ is compact modulo its center, so we can find a subspace $\mathscr{H} \subset \mathscr{S}\left(D_{v}^{2}\right)$ which transforms under $G^{\prime}\left(E_{v}\right)_{+}$as the contragredient to $\left[\pi^{D} \otimes \omega\right](2)$. Taking $\varphi$ in this subspace, the integral $Z_{v}\left(s, f, f^{\prime}, \mathscr{F}_{\varphi}\right)$ reduces to an integral of matrix coefficients of $\mathscr{H}$ against matrix coefficients of $\pi^{D} \otimes \omega$, and these coefficients can certainly be chosen to make the integral nonzero (for a more general version of this argument, cf. [26, Theorem 5.4]).

Thus we may assume $D^{\prime}$ split at $v$. Now, none of the $Z_{v}\left(s, f, f^{\prime}, \mathscr{F}_{\varphi}\right)$ has a pole at $s=0[32, \S 3]$. Combining (6.3.5) and (6.3.8), we may thus conclude that (6.3.10) is equivalent to the nonvanishing of $\Theta_{\psi}\left(\omega \otimes \pi^{D^{\prime}}\right)$. Since the latter is equivalent to the identity $\widetilde{\Theta}\left(\omega \otimes \pi^{D^{\prime}}\right)=\omega^{-1} \otimes \check{\pi}^{D}$, it follows by adjointness that (6.3.10) is equivalent to the nonvanishing of $\Theta_{\psi}\left(G U_{\mathscr{K}}(D) \rightarrow G U_{\mathscr{K}}\left(D^{\prime}\right) ; \omega \otimes \pi^{D}\right)$. But now the family of zeta integrals in (6.3.10) (integrals over $G^{\prime}$ ) depends only on $D_{v}$ and $D_{v}^{\prime}$. Thus it suffices to show that, under the hypotheses of Theorem 4.5 , there exist quaternion algebras $\mathscr{D}$ and $\mathscr{D}^{\prime}$ over $E$, such that

$$
\begin{gathered}
\pi^{\mathscr{D}} \text { and } \pi^{\mathscr{D}^{\prime}} \text { exist, } \mathscr{D}_{v} \cong D_{v}, \mathscr{D}_{v}^{\prime} \cong D_{v}^{\prime}, \mathscr{D} \text { not globally split; } \\
\Theta_{\psi}\left(G U_{\mathscr{K}}(\mathscr{D}) \rightarrow G U_{\mathscr{K}}\left(\mathscr{D}^{\prime}\right) ; \omega \otimes \pi^{\mathscr{D}}\right) \neq 0
\end{gathered}
$$

Let $S\left(D^{\prime}\right)$ be the set of places of $E$ at which $D^{\prime}$ is ramified, and let $\mathscr{D}$ and $\mathscr{D}^{\prime}$ be the quaternion algebras obtained from $D$ and $D^{\prime}$, respectively, by switching the Brauer group invariants at all places in $S\left(D^{\prime}\right)$. Then $\mathscr{D}^{\prime}$ is globally split and $\mathscr{D}$ is not; this is where we use the hypothesis $D \not D^{\prime}$, in order to be able to refer to the simple version 6.3.3 of the Weil-Siegel formula. Now (6.3.12) is 
certainly satisfied; but then (6.3.13) is the special case of Theorem 4.5 for which $\mathscr{D}^{\prime}$ is split, which was proved in $\S 4.5$. This completes the proof of $(6.3 .6)$, thus of Theorem 4.5.

Applying (6.3.9), we obtain

$$
d_{2} \cdot B_{D}\left(\theta_{\varphi}\left(F^{D^{\prime}}\right)\right) \sim_{\overline{\mathbb{Q}}} q^{D^{\prime}}(\pi) \cdot L^{S}\left(\pi_{\mathscr{K}} \otimes \omega, \frac{1}{2}, \mathrm{St}\right) \cdot\left[\prod_{v \in S} Z_{v}\left(s, f, f^{\prime}, \mathscr{F}_{\varphi}\right)\right]_{s=0} .
$$

Write this last product as $Z_{\infty} \cdot Z_{f}$. Now $Z_{\infty}$ depends only on $\varphi_{\infty}, \underline{k}, \chi, D$, and $D^{\prime}$. Thus, if we define $C_{1}=\left(d_{2}\right)^{-1} \cdot Z_{\infty}$, we find

$$
B_{D}\left(\theta_{\varphi}\left(F^{D^{\prime}}\right)\right) \sim_{\overline{\mathbb{Q}}} C_{1} \cdot q^{D^{\prime}}(\pi) \cdot L^{S}\left(\pi_{\mathscr{K}} \otimes \omega, \frac{1}{2}, \mathrm{St}\right) \cdot Z_{f} \cdot
$$

Then (6.3.7), and hence Proposition 5.2.4, will follow from Lemma 6.2.7(ii), once we show that the relevant hypotheses are satisfied. But, on the one hand, the nonarchimedean local components of $\mathscr{F}_{\varphi}$ take values in $\overline{\mathbb{Q}}$ since $\varphi \in$ $\mathscr{S}(\overline{\mathbb{Q}})$; on the other hand, we have chosen $F^{D^{\prime}}$ to belong to the $\overline{\mathbb{Q}}$-rational form $\left[\omega \otimes \pi_{f}^{D^{\prime} \chi^{D^{\prime}}}\right](2)(\overline{\mathbb{Q}})$. This completes the proof.

6.4. The $U(1) \times U\left(D^{\prime}\right)$ lift. We now consider diagram (3.5.3.1) when $V=\mathscr{K}$ and $V^{\prime}=D^{\prime}$; this is the case discussed in $\S 3.6$. Thus $G U_{\mathscr{H}}(V \oplus(-V)) \cong$ $G U_{\mathscr{K}}(s)=G U_{\mathscr{K}}(1,1)$, viewed as the similitude group of the skew-hermitian form $я \cdot S_{1}$. We have the seesaw diagram

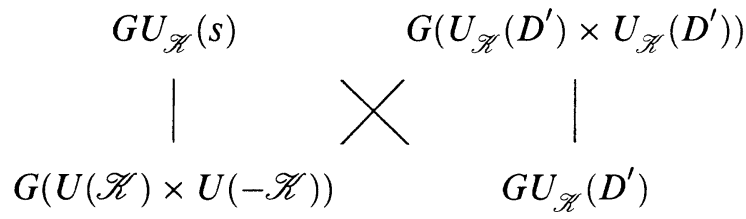

At each place $v$ of $E$ there is an identification $R\left(D^{\prime}, s\right):=R_{\mathscr{K}}\left(V \oplus(-V), V^{\prime}\right)$ $\cong U_{\mathscr{K}_{v}}(s) \rtimes G U\left(D^{\prime}\right)$ (semidirect product). Here $h \in G U\left(D^{\prime}\right)$ acts on $U_{\mathscr{K}_{v}}(s)$, as in $\S 3.2$, by conjugation by $\left(\begin{array}{cc}1 & 0 \\ 0 & \nu(h)\end{array}\right)$, where $\nu=\nu_{D^{\prime}}$. The action $r_{\psi}$ of $R\left(D^{\prime}, s\right)$ on the Schwartz-Bruhat space $\mathscr{S}\left(D_{\mathrm{A}}^{\prime}\right)$ in the Schrödinger model is defined by (3.6.3-5). As we saw in $\S 3.6$, the action of $G\left(U\left(D^{\prime}\right) \times U\left(D^{\prime}\right)\right)$ on $\mathscr{S}\left(D_{\mathrm{A}}^{\prime}\right)$ is determined by an auxiliary splitting $\gamma$. Although the exact form of $\gamma$ does not concern us, we write

$$
\theta_{\varphi}^{\gamma}\left(G(U(\mathscr{K}) \times U(-\mathscr{K})) \rightarrow G\left(U\left(D^{\prime}\right) \times U\left(D^{\prime}\right)\right) ; *\right)
$$

to stress the dependence of the theta liftings on these choices, and

$$
\theta_{\varphi}\left(G U\left(D^{\prime}\right) \rightarrow G U_{\mathscr{K}}(s) ; *\right)
$$

for the other lifting.

Suppose $\varphi \in \mathscr{S}\left(D_{\mathbf{A}}^{\prime}\right)$, and let $\mathscr{F}_{\varphi}(g)=r_{\psi}(g, \alpha) \varphi(0)$ for $g \in G U_{\mathscr{K}}(s, \mathbf{A})$ with $\nu(g)=\alpha \in N_{\mathscr{K} / E}\left(D_{\mathbf{A}}^{\prime, \times}\right)$. Let $P$ be the upper-triangular Borel subgroup of $G U_{\mathscr{K}}(s)$, and let

$$
p(a, b, t)=\left(\begin{array}{cc}
a & b \\
0 & t \cdot\left(a^{l}\right)^{-1}
\end{array}\right) \in P(\mathbf{A}),
$$


with $a \in \mathscr{K}_{\mathbf{A}}^{\times}, t \in E_{\mathbf{A}}^{\times}, b \in E_{\mathbf{A}}$, be the typical element. Then (6.4.2)

$$
\mathscr{F}_{\varphi}(p(a, b, t) \cdot g)=\left\|t^{-1} N_{\mathscr{K} / E}(a)\right\|_{\mathbf{A}} \cdot \mathscr{F}_{\varphi}(g), \quad a \in \mathscr{K}_{\mathbf{A}}^{\times}, t \in E_{\mathbf{A}}^{\times}, b \in E_{\mathbf{A}} .
$$

Thus $f_{\varphi} \in I_{1 / 2, \mathbf{A}}$, in the notation of (6.1.5), with $n=1$. Let $\mathscr{F}_{\varphi}(g, s)=$ $\delta_{1}(g)^{s} \cdot \mathscr{F}_{\varphi}(g)$ for $g \in G U_{\mathscr{H}}(s, \mathbf{A})$. Then $\mathscr{F}_{\varphi}(g, s)$ is a section, in the sense of $\S 6.1$, and we can define the Eisenstein series $E\left(g, s, \mathscr{F}_{\varphi}\right)$. For a general section $\mathscr{F}$ the Eisenstein series $E(g, s, \mathscr{F})$ may have a pole at $s=\frac{1}{2}$. However, $\mathscr{F}_{\varphi}$ is a Weil-Siegel section, and the Weil-Siegel formula in this case asserts:

6.4.3. For all $\varphi \in \mathscr{S}\left(D_{\mathbf{A}}^{\prime}\right), E\left(g, s, \mathscr{F}_{\varphi}\right)$ is holomorphic at $s=\frac{1}{2}$, and

$$
\theta_{\varphi}\left(G U\left(D^{\prime}\right) \rightarrow G U_{\mathscr{H}}(s) ; 1\right)=E\left(g, \frac{1}{2}, \mathscr{F}_{\varphi}\right) .
$$

Again, the analogous assertion for symplectic groups has been proved by Kudla and Rallis. Indeed, the holomorphy at $s=\frac{1}{2}$ is already contained in [22]. This is because, by a simple application of the seesaw diagram (3.5.1.3), $E\left(g, s, \mathscr{F}_{\varphi}\right)$ is just an extension to $G U_{\mathscr{K}}(s)$ of a Weil-Siegel Eisenstein series on $G L(2)$, associated to the dual reductive pair $\left(G L(2), G L\left(D^{\prime}\right)\right)$. It is then not difficult to deduce 6.4.3 from the result in [22] by comparing Fourier expansions of the two sides.

6.4.4. Proof of Proposition 5.3.9. Here we are not concerned with the nonvanishing of the theta lift, so the argument is simpler than in $\S 6.3$. However, we have first to explain the sleight of hand which has replaced the space $\mathscr{S}\left(D(\mathbf{A})^{2}\right)$ of $\S 5$ with the space $S\left(D^{\prime}(\mathbf{A})^{2}\right)$ of $\S 3.6$ and this section. Recall that in $(3.6 .1)$ we have rewritten $(D \oplus(-D)) \otimes_{\mathscr{K}} D^{\prime}$ as

(6.4.5) $\left(V_{1} \oplus V_{2}\right) \otimes_{\mathscr{K}}\left(D^{\prime} \oplus\left(-D^{\prime}\right)\right) \cong V_{1} \otimes_{\mathscr{K}}\left(D^{\prime} \oplus\left(-D^{\prime}\right)\right) \oplus V_{2} \otimes_{\mathscr{K}}\left(D^{\prime} \oplus\left(-D^{\prime}\right)\right)$

This has the effect, in Lemma 3.3, of changing the polarization on the big symplectic space $\mathscr{W}^{\prime \prime}$. The relation between the two Schrödinger models is given by a partial Fourier transform $P: \mathscr{S}\left(D(\mathbf{A})^{2}\right) \stackrel{\sim}{\rightarrow} \mathscr{S}\left(D^{\prime}(\mathbf{A})^{2}\right)$, cf. [28, p. 32]. The integral defining $P$ breaks up as a product of local integrals $P_{v}: \mathscr{S}\left(D_{v}^{2}\right) \stackrel{\sim}{\rightarrow} \mathscr{S}\left(D_{v}^{\prime, 2}\right)$. Then for all finite $v, P_{v}$ preserves the space of $\mathbb{Q}^{\text {ab }}$-valued Schwartz-Bruhat functions. Let $P_{\infty}: \mathscr{S}\left(D(\mathbb{R})^{2}\right) \stackrel{\sim}{\rightarrow} \mathscr{S}\left(D^{\prime}(\mathbb{R})^{2}\right)$ be the archimedean constituent of $P$, and let $\varphi_{\infty, D^{\prime}}^{\infty}=P_{\infty}\left(\varphi_{\infty}\right)$. Then

$$
\mathscr{S}^{\prime}(\overline{\mathbb{Q}}):=P(\mathscr{S}(\overline{\mathbb{Q}}))=\overline{\mathbb{Q}} \cdot \varphi_{\infty, D^{\prime}} \otimes \mathscr{S}\left(D^{\prime}\left(\mathbf{A}^{f}\right)^{2}\right)(\overline{\mathbb{Q}}) \subset \mathscr{S}\left(D^{\prime}(\mathbf{A})^{2}\right)
$$

is a $\mathscr{G}^{\prime}\left(\mathbf{A}^{f}\right)$-invariant $\overline{\mathbb{Q}}$-form of $\mathscr{S}\left(D^{\prime}(\mathbf{A})^{2}\right)$. Furthermore, as forms on $\mathscr{G}^{\prime}$,

$$
\theta_{\varphi}\left(\eta_{1} \otimes \eta_{2}(2)\right)=\theta_{P(\varphi)}\left(\eta_{1} \otimes \eta_{2}(2)\right),
$$

since the theta lifting never depends on the model. It thus suffices to compute $\widetilde{B}_{D^{\prime}}\left(\theta_{P(\varphi)}\left(\eta_{1} \otimes \eta_{2}(2)\right)\right)$.

Write $\varphi_{\infty, D^{\prime}}=\varphi_{\infty, 1} \otimes \varphi_{\infty, 2}$, with $\varphi_{\infty, i} \in \mathscr{S}(D(\mathbb{R})), i=1,2$, relative to the splitting $\left(V_{1} \oplus V_{2}\right) \otimes_{\mathscr{K}}\left(D^{\prime} \oplus\left(-D^{\prime}\right)\right) \cong V_{1} \otimes_{\mathscr{K}}\left(D^{\prime} \oplus\left(-D^{\prime}\right)\right) \oplus V_{2} \otimes_{\mathscr{K}}\left(D^{\prime} \oplus\left(-D^{\prime}\right)\right)$ of (6.4.5). This is possible because $\varphi_{\infty}$, and hence $\varphi_{\infty, D^{\prime}}$, was chosen to be 
of pure $U\left(V_{1}(\mathbb{R})\right) \times U\left(V_{2}(\mathbb{R})\right)$-type. Similarly, write $\mathscr{S}^{\prime}(\overline{\mathbb{Q}})=\mathscr{S}_{1}(\overline{\mathbb{Q}}) \otimes \mathscr{S}_{2}(\overline{\mathbb{Q}})$, where $\mathscr{S}_{i}(\overline{\mathbb{Q}})=\overline{\mathbb{Q}} \cdot \varphi_{\infty, i} \otimes \mathscr{S}\left(D^{\prime}\left(\mathbf{A}^{f}\right)\right)(\overline{\mathbb{Q}})$.

If $\eta$ is any Hecke character of $\mathscr{K}$, write $\eta \otimes \eta^{-1}$ for the corresponding Hecke character of $G(U(\mathscr{K}) \times U(-\mathscr{K})) \subset\left(\mathscr{K}^{\times}\right)^{2}$, and for $\varphi \in \mathscr{S}\left(D^{\prime}(\mathbf{A})\right)$, we can define the automorphic form on $G\left(U_{\mathscr{H}}\left(D^{\prime}\right) \times U_{\mathscr{K}}\left(D^{\prime}\right)\right)$ :

$$
\theta_{\varphi}\left(\eta \otimes \eta^{-1}\right)=\theta_{\varphi}\left(G(U(\mathscr{K}) \times U(-\mathscr{K})), G\left(U_{\mathscr{K}}\left(D^{\prime}\right) \times U_{\mathscr{K}}\left(D^{\prime}\right) ; \eta \otimes \eta^{-1}\right) .\right.
$$

It will be enough to show that, for $i=1,2$, there exists $C_{2} \in \mathbb{C}^{\times}$such that

$$
B_{D^{\prime}}\left(\theta_{\varphi}\left(\eta_{i} \otimes \eta_{i}^{-1}\right)\right) \sim_{\overline{\mathbb{Q}}} C_{2} \cdot L_{\mathscr{K}}\left(\tilde{\eta}_{i}, 1\right), \quad \forall \varphi \in \mathscr{S}_{i}(\overline{\mathbb{Q}}),
$$

with $B_{D^{\prime}}$ defined as in (5.2.1).

Now apply seesaw reciprocity for the diagram (6.4.1). In this case, $\mathscr{G} \mathscr{U}^{\prime}(\mathbf{A})_{0}$ $=\mathscr{G} \mathscr{U}^{\prime}(\mathbf{A})=G U_{\mathscr{K}}\left(D^{\prime}(\mathbf{A})\right), \mathscr{G} \mathscr{V}(\mathbf{A})_{0}=\mathscr{G} \mathscr{V}(\mathbf{A})=G\left(U_{\mathscr{H}}\left(V_{i}(\mathbf{A})\right) \times U_{\mathscr{H}}\left(-V_{i}(\mathbf{A})\right)\right)$. We have $F_{\mathscr{U}^{\prime}}=1, F_{\mathscr{V}}=\eta_{i} \otimes \eta_{i}^{-1}$. Then 6.4 .3 yields

$$
\begin{aligned}
B_{D^{\prime}}\left(\theta_{\varphi}\left(\eta_{i} \otimes \eta_{i}^{-1}\right)\right) & =\left\langle\theta_{\varphi}\left(F_{\mathscr{V}}\right), F_{\mathscr{U}^{\prime}}\right\rangle_{\mathscr{U}^{\prime}}=\left\langle\theta_{\varphi}\left(F_{\mathscr{\ell ^ { \prime }}}\right), F_{\mathscr{V}}\right\rangle_{\mathscr{V}} \\
& =\left\langle E\left(g, \frac{1}{2}, \mathscr{F}_{\varphi}\right), \eta_{i} \otimes \eta_{i}^{-1}\right\rangle_{\mathscr{V}} .
\end{aligned}
$$

Applying the basic identity (6.2.3) and (6.2.8), we find (6.4.8)

$$
d_{1}(0) \cdot B_{D^{\prime}}\left(\theta_{\varphi}\left(\eta_{i} \otimes \eta_{i}^{-1}\right)\right)=\left\langle\eta_{i}, \eta_{i}^{-1}\right\rangle_{V_{i}} \cdot L_{\mathscr{H}}^{S}\left(\tilde{\eta}_{i}, 1\right) \cdot\left[\prod_{v \in S} Z_{v}\left(s, \eta_{i}, \eta_{i}^{-1}, \mathscr{F}_{\varphi}\right)\right]_{s=1 / 2} .
$$

Now $\left\langle\eta_{i}, \eta_{i}^{-1}\right\rangle_{V_{i}}$ is the volume of $\mathscr{K}_{\mathbf{A}}^{\times} / \mathscr{K}^{\times} \cdot E_{\mathbf{A}}^{\times}$, and $d_{1}(0)=\zeta_{E}^{S}(2)$. We complete the proof by verifying, as in $\S 6.3$, that the hypotheses of Lemma 6.2.7 are satisfied.

\section{Special values of $L$-Functions ANd COMPLements}

7.1. Let $\pi$ be as in Theorem 2.6.1. We begin by returning to formula (5.7.8). Thus we suppose $D^{\prime}=D(\varnothing), D$ arbitrary, and $\pi$ and $\omega$ chosen to satisfy the hypotheses of Theorem 4.5. More precisely, Corollary 4.4.5 and Theorem 4.5 together say that, for each $\pi$ such that $\pi^{D^{\prime}}$ and $\pi^{D}$ exist, and each collection of local characters $\left\{\chi_{v}\right\}$ as in Corollary 4.4.5, there exists $\omega$ satisfying conditions (i)-(iii) of Corollary 4.4.5 such that the conclusions of Theorem 4.5 are valid. Then formula (5.7.8) asserts

$$
C_{4}(\underline{k}, \Sigma(D), \varnothing) \cdot L^{\mathrm{mot}}\left(\pi_{\mathscr{K}} \otimes \omega, 1\right) \sim_{\bar{Q}} q^{D}(\pi) \cdot p_{\mathscr{K}}(\tilde{\omega}, l(\Phi-\Psi)),
$$

where $\omega$ is compatible with $\imath \Phi, \Psi=\left\{\tau \in \Phi|\tau|_{E} \in \Sigma(D)\right\}$, and

$$
L^{\mathrm{mot}}\left(\pi_{\mathscr{H}} \otimes \omega, s\right)=L\left(\pi_{\mathscr{K}} \otimes \omega, s-\frac{1}{2}\right)
$$

is the motivic normalization of the $L$-function:

$$
L^{\mathrm{mot}}\left(\pi_{\mathscr{K}} \otimes \omega, s\right)=L(\pi \otimes \pi(\omega, \mathscr{K}), s) .
$$


In this case we can break down the unknown constant

$$
C_{4}(\underline{k}, \Sigma(D), \varnothing)=C_{1} / C_{3}=C_{1} /(2 \pi i)^{2 d} \cdot C_{2}
$$

by (5.7.7) and the preceding arguments. Here $C_{2}=C_{2}\left(\varphi_{\infty}, \underline{k}\left(\eta_{1}\right), \underline{k}\left(\eta_{2}\right), \Sigma\left(D^{\prime}\right)\right)$ $=C_{2}\left(\varphi_{\infty}, \underline{k}\left(\eta_{1}\right), \underline{k}\left(\eta_{2}\right), \varnothing\right)$ is the constant of Proposition 5.3.8, and $C_{1}=C_{1}\left(\varphi_{\infty}, \underline{k}, \chi, \varnothing, \Sigma(D)\right)$ is the constant of Proposition 5.2.4. Now $C_{1}=$ $\left(d_{2}\right)^{-1} Z_{\infty} \sim_{\mathbb{Q}} G\left(\varepsilon_{\mathscr{K}}\right)^{-1}(2 \pi i)^{-3 d} \cdot Z_{\infty, 1}$ by the construction in $\S 6.3$, where $Z_{\infty, 1}$ is the product of archimedean local zeta integrals. On the other hand, in the notation of (6.4.8),

$$
C_{2}=d_{1}(0)^{-2} \cdot\left(\prod_{j=1}^{d} Z_{j}\left(s, \eta_{1},\left(\eta_{1}\right)^{-1}, \mathscr{F}_{\varphi_{1}}\right) \cdot Z_{j}\left(s, \eta_{2},\left(\eta_{2}\right)^{-1}, \mathscr{F}_{\varphi_{2}}\right)\right)_{s=\frac{1}{2}},
$$

where the product is taken over the archimedean places and $d_{1}(0)=\zeta_{E}^{S}(2) \sim_{\mathbb{Q}}$ $(2 \pi i)^{2 d}$. In other words, letting $Z_{\infty, 2}$ be the product of local zeta integrals, we have $C_{2} \sim_{\mathbb{Q}}(2 \pi i)^{-4 d} \cdot Z_{\infty, 2}$, or

$$
G\left(\varepsilon_{\mathscr{K}}\right) \cdot C_{4}(\underline{k}, \Sigma(D), \varnothing) \sim_{\mathbb{Q}}(2 \pi i)^{-d} \cdot Z_{\infty, 1} / Z_{\infty, 2} \sim_{\mathbb{Q}} \prod_{j=1}^{d} C_{4, j},
$$

where, if $Z_{j, i}$ is the local integral (or product of integrals) in $Z_{\infty, i}, i=1,2$, $j=1, \ldots, d$, then $C_{4, j}=(2 \pi i)^{-1} \cdot Z_{j, 1} / Z_{j, 2}$.

The point of (7.1.3) is that, at least up to algebraic factors (depending on the choice of $\mathrm{CM}$ point), $C_{4}$ is a product of the local zeta integrals which can be computed already for $E=\mathbb{Q}$. When $E=\mathbb{Q}$, there are essentially only two cases: $\Sigma(D)=\Sigma$ and $\Sigma(D)=\varnothing$. We write $C_{4}(\underline{k}, \Sigma(D), \varnothing)=$ $C_{4}\left(\underline{k}, \Sigma(D)_{E}, \varnothing_{E}\right)$, to stress dependence on $E$. Then

$$
C_{4}\left(\underline{k}, \Sigma(D)_{E}, \varnothing_{E}\right) \sim_{\overline{\mathbb{Q}}} \prod_{j \in \Sigma(D)} C\left(k_{j}, \Sigma_{\mathbb{Q}}, \varnothing_{\mathbb{Q}}\right) \cdot \prod_{j \in \Sigma(D)^{\prime}} C\left(k_{j}, \varnothing_{\mathbb{Q}}, \varnothing\right) .
$$

We work out the two factors in turn, for arbitrary $E$.

First suppose $\Sigma(D)=\Sigma$. Hypothesis (ii) of Corollary 4.4.5 and Corollary 4.4.1 then imply that $k_{j}>k(\omega)_{j}$ for all $j$. Then Theorem 3.5 .1 of [9, I] asserts:

$$
L^{\mathrm{mot}}\left(\pi_{\mathscr{K}} \otimes \omega, 1\right)=L(\pi \otimes \pi(\omega, \mathscr{K}), 1) \sim_{\overline{\mathbb{Q}}}(2 \pi i)^{d} \nu^{\Sigma}(\pi),
$$

cf. the arguments in $\S \S 1.8-9$. On the other hand, (7.1.1) states

$$
C_{4}\left(\underline{k}, \Sigma_{E}, \varnothing_{E}\right) \cdot L^{\mathrm{mot}}\left(\pi_{\mathscr{K}} \otimes \omega, 1\right) \sim_{\overline{\mathbb{Q}}} q^{D}(\pi) \sim_{\overline{\mathbb{Q}}} \nu^{\Sigma}(\pi),
$$

where $\nu^{\Sigma}(\pi)$ is the invariant mentioned in $\S 1.8$; the second relation is $[9, \mathrm{I}$, (1.6.5)]. Thus

$$
C_{4}\left(\underline{k}, \Sigma_{E}, \varnothing_{E}\right) \sim_{\overline{\mathbb{Q}}}(2 \pi i)^{-d} .
$$


Now suppose $\Sigma(D)=\varnothing$. Hypothesis (ii) of Corollary 4.4.5 and Corollary 4.4.1 then imply that $k_{j}<k(\omega)_{j}$ for all $j$. Then Theorem 3.5.1 of [9, I] asserts

$$
L^{\mathrm{mot}}\left(\pi_{\mathscr{K}} \otimes \omega, 1\right)=L(\pi \otimes \pi(\omega, \mathscr{K}), 1) \sim_{\overline{\mathbb{Q}}}(2 \pi i)^{d} \nu^{\Sigma}(\omega, \mathscr{K}) .
$$

On the other hand, (7.1.1) states

$$
C_{4}\left(\underline{k}, \varnothing_{E}, \varnothing_{E}\right) \cdot L^{\mathrm{mot}}\left(\pi_{\mathscr{K}} \otimes \omega, 1\right) \sim_{\overline{\mathbb{Q}}} p_{\mathscr{K}}(\tilde{\omega}, l \Phi) \sim_{\overline{\mathbb{Q}}} \nu^{\Sigma}(\omega, \mathscr{K}),
$$

where the second relation is the special case of Lemma 1.9.5 for which $I^{\prime}=\varnothing$, $I=\Sigma$. Thus

$$
C_{4}\left(\underline{k}, \varnothing_{E}, \varnothing_{E}\right) \sim_{\bar{Q}}(2 \pi i)^{-d} .
$$

Now it follows from (7.1.5-6) applied to $E=\mathbb{Q}$ and (7.1.4) that

$$
C_{4}\left(\underline{k}, \Sigma(D)_{E}, \varnothing_{E}\right) \sim_{\bar{Q}}(2 \pi i)^{-d} \text { for all } D .
$$

Thus (7.1.1) yields

7.1.8. Proposition. Let $\omega$ be any motivic Hecke character such that $k(\omega)_{j}>k_{j}$ for $j \in \Sigma(D)^{\prime}, k(\omega)_{j}<k_{j}$ for $j \in \Sigma(D)$. Then under the hypotheses of Theorem 2.6.1,

$$
L^{\mathrm{mot}}\left(\pi_{\mathscr{K}} \otimes \omega, 1\right)=L(\pi \otimes \pi(\omega, \mathscr{K}), 1) \sim_{\overline{\mathbb{Q}}}(2 \pi i)^{d} \cdot q^{D}(\pi) \cdot p_{\mathscr{H}}(\tilde{\omega}, l(\Phi-\Psi)) .
$$

Proof. The proposition follows from (7.1.1) and (7.1.7), provided $L^{\mathrm{mot}}\left(\pi_{\mathscr{K}} \otimes \omega, 1\right) \neq 0$, but if $L^{\mathrm{mot}}\left(\pi_{\mathscr{K}} \otimes \omega, 1\right)=0$ there is nothing to prove.

We remark that a $\operatorname{Gal}(\overline{\mathbb{Q}} / \mathbb{Q})$-equivariant version of this proposition can be proved by analyzing the global zeta integral of $\S 6$ on $U_{\mathscr{H}}(D)$, along the lines of $[37,5]$. This will appear in a forthcoming paper.

When $k(\omega)_{j} \geq 2$ for all $j$, this proposition is included among Shimura's results in [42]. However, when $k_{j}=2$ for some $j \in \Sigma(D)$, it is essential to allow $k(\omega)_{j}=1$ in order to prove our main theorem.

7.2. Proof of Shimura's Theorem 2.6.3. Note that Shimura's Theorem 2.6.3 enters into the argument in $\S 5$ in its application to $D^{\prime}$. When $D^{\prime}=D^{\prime}(\varnothing)$ Theorem 2.6.3 is trivial. Thus formula (7.1.1) does not depend at all on Theorem 2.6.3. We now show how to derive Theorem 2.6.3 from Proposition 7.1.8 and Lemma 1.9.5. The character in the statement of the theorem will be denoted $\eta$. Let $\pi=\pi(\eta, \mathscr{K})$. Then Proposition 7.1.8 asserts

$$
L(\pi(\eta, \mathscr{K}) \otimes \pi(\omega, \mathscr{K}), 1) \sim_{\overline{\mathbb{Q}}}(2 \pi i)^{d} \cdot q^{D}(\pi) \cdot p_{\mathscr{H}}(\tilde{\omega}, l(\Phi-\Psi)) .
$$

We choose $\omega$ subject to the hypotheses $k(\omega)_{j}>k(\eta)_{j}$ for $j \in \Sigma(D)^{\prime}, k(\omega)_{j}<$ $k(\eta)_{j}$ for $j \in \Sigma(D)$, and $L(\pi(\eta, \mathscr{K}) \otimes \pi(\omega, \mathscr{K}), 1) \neq 0$; this is possible by Corollary 4.4.1. Then applying Theorem 3.5.1 of $[9, \mathrm{I}]$, as above, we obtain

$$
(2 \pi i)^{d} \cdot \nu^{\Sigma(D)}(\eta, \mathscr{K}) \cdot \nu^{\Sigma(D)^{\prime}}(\omega, \mathscr{K}) \sim_{\overline{\mathbb{Q}}}(2 \pi i)^{d} \cdot q^{D}(\pi) \cdot p_{\mathscr{H}}(\tilde{\omega}, l(\Phi-\Psi)) .
$$

But Lemma 1.9.5, with $I^{\prime}=\Sigma(D), I=\Sigma(D)^{\prime}$, implies that

$$
\nu^{\Sigma(D)}(\eta, \mathscr{K}) \cdot \nu^{\Sigma(D)^{\prime}}(\omega, \mathscr{K}) \sim_{\overline{\mathbb{Q}}} p_{\mathscr{K}}(\tilde{\eta}, \Psi) \cdot p_{\mathscr{\not}}(\tilde{\omega}, l(\Phi-\Psi)) .
$$

Theorem 2.6.3 follows immediately from the last two formulas. 
7.3. Proof of Shimura's case of Theorem 2.6.1. Let $\Sigma=I \amalg J$ and let $q^{I}=$ $q^{D(I)}(\pi), q^{J}=q^{D(J)}(\pi), q^{\Sigma}=q^{D(\Sigma)}(\pi)$, where $D(\Sigma)=M(2, E)$. We want to prove that

$$
q^{I} \cdot q^{J} \sim_{\overline{\mathbb{Q}}} q^{\Sigma}=\nu^{\Sigma}(\pi)
$$

As above, we derive the relation from Proposition 7.1.8. In the first place, let $\omega^{\prime}$ (resp. $\omega$ ) be a Hecke character satisfying (5.5.5-6) for $D^{\prime}=D(\varnothing), D=D(I)$, and $\pi$ (resp. $D^{\prime}=D(\varnothing), D=D(J)$, and $\check{\pi}$ ). Assume both $\omega$ and $\omega^{\prime}$ compatible with the CM type $\Phi$. For any $I^{\prime} \subset \Sigma$, let $\Phi\left(I^{\prime}\right)=\left\{\tau \in \Phi|\tau|_{E} \in I^{\prime}\right\}$. Then

$$
\begin{aligned}
& 0 \neq L\left(\pi \otimes \pi\left(\omega^{\prime}, \mathscr{K}\right), 1\right) \sim_{\overline{\mathbb{Q}}}(2 \pi i)^{d} \cdot q^{I} \cdot p_{\mathscr{K}}\left(\tilde{\omega}^{\prime}, l \Phi(J)\right) \\
& 0 \neq L(\check{\pi} \otimes \pi(\omega, \mathscr{K}), 1) \sim_{\overline{\mathbb{Q}}}(2 \pi i)^{d} \cdot q^{J} \cdot p_{\mathscr{K}}(\tilde{\omega}, l \Phi(I)) .
\end{aligned}
$$

Here we are using the fact that $\check{\pi}=\pi \otimes \xi_{\pi}^{-1}$, so $q^{D(J)}(\pi)=q^{D(J)}(\check{\pi})$ by $(2.5 .4)$. On the other hand, Theorem 3.5 .1 of $[9, \mathrm{I}]$ asserts

$$
\begin{aligned}
& L\left(\pi \otimes \pi\left(\omega^{\prime}, \mathscr{K}\right), 1\right) \sim_{\overline{\mathbb{Q}}}(2 \pi i)^{d} \cdot \nu^{I}(\pi) \cdot \nu^{J}\left(\omega^{\prime}, \mathscr{K}\right) ; \\
& L(\check{\pi} \otimes \pi(\omega, \mathscr{K}), 1) \sim_{\overline{\mathbb{Q}}}(2 \pi i)^{d} \cdot \nu^{J}(\check{\pi}) \cdot \nu^{J}(\omega, \mathscr{K}) .
\end{aligned}
$$

Comparing (7.3.2) and (7.3.3), we obtain

$$
\begin{gathered}
q^{I} \cdot p_{\mathscr{K}}\left(\tilde{\omega}^{\prime}, l \Phi(J)\right) \sim \sim_{\overline{\mathbb{Q}}} \nu^{I}(\pi) \cdot \nu^{J}\left(\omega^{\prime}, \mathscr{K}\right) ; \\
q^{J} \cdot p_{\mathscr{K}}(\tilde{\omega}, l \Phi(I)) \sim_{\overline{\mathbb{Q}}} \nu^{J}(\check{\pi}) \cdot \nu^{I}(\omega, \mathscr{K}) .
\end{gathered}
$$

Multiplying the two relations in (7.3.4), we obtain

$$
\begin{aligned}
& q^{I} \cdot q^{J} \cdot p_{\mathscr{K}}\left(\tilde{\omega}^{\prime}, l \Phi(J)\right) \cdot p_{\mathscr{K}}(\tilde{\omega}, l \Phi(I)) \sim_{\overline{\mathbb{Q}}} \nu^{I}(\pi) \cdot \nu^{J}(\check{\pi}) \cdot \nu^{J}\left(\omega^{\prime}, \mathscr{K}\right) \cdot \nu^{I}(\omega, \mathscr{K}) \\
& \sim_{\overline{\mathbb{Q}}} \nu^{\Sigma}(\pi) \cdot \nu^{J}\left(\omega^{\prime}, \mathscr{K}\right) \cdot \nu^{I}(\omega, \mathscr{K})
\end{aligned}
$$

by Proposition 1.5 .6 of $[9, \mathrm{I}]$.

On the other hand, $1=\xi_{\pi} \cdot \xi_{\omega}=\xi_{\check{\pi}} \cdot \xi_{\omega^{\prime}}$, so $\xi_{\omega} \cdot \xi_{\omega^{\prime}}=1$. It follows easily that $\omega$ and $\omega^{\prime}$ satisfy the hypotheses of Lemma 1.8.9, with $I^{\prime}=J$. Thus

$$
p_{\mathscr{K}}\left(\tilde{\omega}^{\prime},{ }_{l} \Phi(J)\right) \cdot p_{\mathscr{K}}\left(\tilde{\omega},{ }_{l} \Phi(I)\right) \sim_{\overline{\mathbb{Q}}} \nu^{J}\left(\omega^{\prime}, \mathscr{K}\right) \cdot \nu^{I}(\omega, \mathscr{K}) .
$$

Comparing (7.3.6) with (7.3.5), we obtain (7.3.1). As above, we remark that this proof does not depend on Shimura's results.

In exactly the same way, one can reprove Shimura's theorem [42, Theorem 5.3] expressing the special values of Rankin-Selberg convolutions $L\left(\pi \otimes \pi^{\prime}, s\right)$ in terms of the $q^{D}(\pi)$ and $q^{D^{\prime}}\left(\pi^{\prime}\right)$, by comparing Proposition 7.1.8 with Theorem 3.5.1 of $[9, \mathrm{I}]$ for $\pi$ and $\pi^{\prime}$ and variable Hecke characters $\omega$. We postpone this to a forthcoming paper with Garrett, where we will have access to a more precise version of Proposition 7.1.8. 
7.4. We continue to assume $\pi$ satisfies the hypotheses of Theorem 2.6.1. Let $\mathscr{K}$ be any $C M$ quadratic extension of $E$, and consider the standard $L$-function $L^{\text {mot }}\left(\pi_{\mathscr{H}}, s\right)$, normalized motivically. Then

$$
L^{\mathrm{mot}}\left(\pi_{\mathscr{K}}, s\right)=L(\pi \otimes \pi(1, \mathscr{K}), s),
$$

where 1 is the trivial Hecke character of $\mathscr{K}$ and the tensor product $L$-function is given its standard normalization. Of course, $\pi(1, \mathscr{K})$ is an Eisenstein representation, and $L^{\mathrm{mot}}\left(\pi_{\mathscr{K}}, s\right)=L\left(\pi, s-\frac{1}{2}\right) \cdot L\left(\pi \otimes \varepsilon_{\mathscr{K}}, s-\frac{1}{2}\right)$ (Jacquet-Langlands normalization).

The critical values of the right-hand side of (7.4.1) have been studied by Shimura [37]; it suffices to remark that $k_{j}>k(1)_{j}=1$ for all $j$, by hypothesis. The principal term in the special value is just the quadratic period $q^{\Sigma}(\pi)=\nu^{\Sigma}(\pi)$. Let $q^{j}(\pi)=q^{D(j)}(\pi)$, with $D(j)$ the quaternion algebra ramified precisely at $\sigma_{j}$ and $v_{0}$, as in the proof of 2.6.6. Applying our factorization theorem (2.6.1), Shimura's Theorem 4.2 can be translated as follows:

7.4.2. Theorem. Let $m$ be an integer such that

$$
\frac{1}{2}(2-r) \leq m \leq \frac{1}{2}(1-r)+k_{j}-1, \quad j=1, \ldots, d .
$$

Set $\lambda=2 m-1+r$. Then

$$
L^{\mathrm{mot}}\left(\pi_{\mathscr{K}}, m\right) \sim_{\overline{\mathbb{Q}}}(2 \pi i)^{d \lambda} \cdot i^{d r} \cdot G\left(\xi_{\pi} \cdot \varepsilon_{\mathscr{K}}\right) \cdot \prod_{j=1}^{d} q^{j}(\pi) .
$$

Here $G\left(\xi_{\pi} \cdot \varepsilon_{\mathscr{K}}\right)$ is a Gauss sum, defined as in [9, I, §3.5]. The power of $i$ and the Gauss sum are algebraic numbers, and their inclusion in the statement of the theorem is simply an expression of optimism. The identification of the critical range for $m$ and the power of $(2 \pi i)$ are derived from Shimura's Theorem 4.2 as in [9, I, Remark 3.5.10].

As a special case, we can consider $\pi^{D(j)}$ occurring in the representation generated by invariant differentials on a factor $A(\pi, j)$ of the Jacobian of the Shimura curve $\mathscr{M}_{j}$ attached to the quaternion algebra $D(j)$. Then $k_{j}=2$ for all $j, r=0$, and $m=1$ is the only critical point. In this case $q^{j}(\pi)$ is the volume of the period lattice of $A(\pi, j)$, up to an algebraic multiple. Denote this volume $v(A(\pi, j))$. Then for any $\tau \in \operatorname{Aut}(\mathbb{C}), v\left(A(\pi, j)^{\tau}\right) \sim_{\overline{\mathbb{Q}}} v(A(\pi, \tau(j)))$, where $\operatorname{Aut}(\mathbb{C})$ acts on $\Sigma$ by permutation, cf. (2.1.1). Let $A(\pi, j)_{\mathscr{K}}$ denote the base change of $A(\pi, j)$ to $\mathscr{K}$. We find

$$
\begin{aligned}
L^{\mathrm{mot}}\left(\pi_{\mathscr{K}}, 1\right) & =L\left(A(\pi, j)_{\mathscr{K}}, 1\right) \sim_{\overline{\mathbb{Q}}}(2 \pi i)^{d} \cdot i^{d r} \cdot G\left(\varepsilon_{\mathscr{K}}\right) \cdot \prod_{j=1}^{d} v(A(\pi, j)), \\
& \sim_{\overline{\mathbb{Q}}}(2 \pi i)^{d} \cdot i^{d r} \cdot G\left(\varepsilon_{\mathscr{K}}\right) \cdot \prod_{\tau} v\left(A(\pi, j)^{\tau}\right),
\end{aligned}
$$

where $\tau$ runs through a set of coset representations for $\operatorname{Aut}\left(\mathbb{C} / \sigma_{j}(E)\right)$ in $\operatorname{Aut}(\mathbb{C})$. It is easy to verify that $G\left(\varepsilon_{\mathscr{K}}\right) \sim_{\mathbb{Q}}\left(d_{\mathscr{K}} / d_{E}\right)^{1 / 2}$. Formula (7.4.3) may thus be regarded as the transcendental part of the Birch-Swinnerton-Dyer conjecture for $A(\pi, j)$, viewed as an abelian variety over $\mathscr{K}$. 
7.5. It remains to explain what our methods provide in the absence of a local hypothesis at $v_{0}$. Then we can only work with quaternion algebras $D$ unramified outside $\infty$. Suppose $D, D^{\prime}, \pi$, and $\omega$ are taken to satisfy the hypotheses of $\S \S 5.1-5.6$; in particular, $\Sigma(D) \equiv \Sigma\left(D^{\prime}\right) \equiv d(\bmod 2)$. Then the arguments up to (5.7.7) remain valid. The case of $d$ even is covered by the second part of Theorem 2.6.5. Thus we assume $d$ odd. In this case there is no formula like (5.7.8) for the value $L\left(\pi_{\mathscr{K}} \otimes \omega, \frac{1}{2}\right)$, and we cannot proceed.

However, Theorem 3.5.1 of [9, I] provides a different formula for $L\left(\pi_{\mathscr{K}} \otimes \omega, \frac{1}{2}\right)=L(\pi \otimes \pi(\omega, \mathscr{K}), 1)$ :

$$
L\left(\pi_{\mathscr{K}} \otimes \omega, \frac{1}{2}\right) \sim_{\overline{\mathbb{Q}}}(2 \pi i)^{d} \cdot \nu^{\Sigma(D)-\Sigma\left(D^{\prime}\right)}(\pi) \cdot \nu^{\Sigma(D)^{\prime} \amalg \Sigma\left(D^{\prime}\right)}(\omega, \mathscr{K}) .
$$

Now Theorem 1 of $[9$, II] provides the relation

$$
p_{\mathscr{K}}\left(\tilde{\omega}, \imath \Psi^{\prime} \amalg l(\Phi-\Psi)\right) \sim_{\overline{\mathbb{Q}}} \nu^{\Sigma(D)^{\prime} \amalg \Sigma\left(D^{\prime}\right)}(\omega, \mathscr{K}) .
$$

The only proof I know of (7.5.2) uses relations derived in [9, I, §4] from the formula of [13] for the central critical value of the triple product $L$-function. Writing

$$
L\left(\pi_{\mathscr{K}} \otimes \omega, \frac{1}{2}\right) \sim_{\overline{\mathbb{Q}}}(2 \pi i)^{d} \cdot \nu^{\Sigma(D)-\Sigma\left(D^{\prime}\right)}(\pi) \cdot p_{\mathscr{K}}\left(\tilde{\omega}, l \Psi^{\prime} \amalg l(\Phi-\Psi)\right)
$$

and continuing as in $\S 5.7$, we eventually obtain the period relations of the following proposition, which we state in the most general case.

7.5.4. Proposition. Suppose $\pi$ satisfies the hypotheses of Theorem 2.6.5, with the exception of the local hypotheses at $v_{0}$. Let $I^{\prime} \subset I \subset \Sigma$ be subsets such that $|\Sigma-I| \equiv\left|\Sigma-I^{\prime}\right| \equiv 0(\bmod 2)$. Define $D\left(I^{\prime}\right), D(I)$ as in 2.6.2(b). Then $\pi^{D(I)}$ and $\pi^{D\left(I^{\prime}\right)}$ exist, and

$$
q^{D(I)}(\pi) \sim_{\overline{\mathbb{Q}}} q^{D\left(I^{\prime}\right)}(\pi) \cdot \nu^{I-I^{\prime}}(\pi) .
$$

More generally, let $r=[d / 2]$, and choose any partition $\Sigma=J_{1} \amalg \cdots \amalg J_{r} \amalg I$, where all the $J_{i}$ have cardinality 2 and $|I|=0$ or 1 according as $d$ is even or odd. Then

$$
\nu^{\Sigma}(\pi)=q^{\Sigma}(\pi) \sim_{\overline{\mathbb{Q}}} \nu^{J_{1}}(\pi) \cdots \cdots \nu^{J_{r}}(\pi) \cdot q^{D(I)}(\pi) .
$$

Proof. The first relation follows from (7.5.3) by the arguments of 5.7; the latter relation is proved by induction on $|I|$. The relation $\nu^{\Sigma}(\pi)=q^{\Sigma}(\pi)$ is Proposition 1.5 .6 of $[9, \mathrm{I}]$.

Theorem 1 of $\left[9\right.$, II] also includes the relation $\nu^{J_{i}}(\pi) \sim_{\overline{\mathbb{Q}}} q^{D\left(J_{i}\right)}(\pi)$ if $d$ is even; thus one can always factor $q^{\Sigma}(\pi)$ as a product of quaternionic Petersson norms in the case of even degree.

\section{APPENDIX: COMPLEX CONJUGATION OF CM PERIODS}

With notation as in $\S 2.4$, we need to prove the formulas

$$
p_{\mathscr{K}}\left(\overline{\omega^{l}}, \Psi\right) \sim_{E_{\Psi} \cdot E(\omega)} \overline{p_{\mathscr{K}}(\omega, \Psi)} ;
$$




$$
\begin{aligned}
\text { For } \sigma & \in \operatorname{Gal}\left(\overline{\mathbb{Q}} / E_{\Psi}\right),\left[p_{\mathscr{H}}\left(\overline{\omega^{l}}, \Psi\right) / \overline{p_{\mathscr{H}}(\omega, \Psi)}\right]^{\sigma} \\
& =\left[p_{\mathscr{H}}\left(\left(\bar{\omega}^{\sigma}\right)^{l}, \Psi\right) / \bar{p}_{\mathscr{H}}\left(\omega^{\sigma}, \Psi\right)\right] .
\end{aligned}
$$

We observe that, up to periods of the form $p_{\mathscr{K}}(\eta, \Psi)$ for $\eta$ a character of finite order, the periods $p_{\mathscr{H}}(\omega, \Psi)$ can be expressed multiplicatively in terms of periods of the form $p_{\mathscr{K}^{\prime}}\left(\omega^{\prime}, \Phi^{\prime}\right)$, where $\left\{\left(\mathscr{K}^{\prime}, \Phi^{\prime}\right)\right\}$ is a set of CM fields with corresponding $\mathrm{CM}$ types and $\omega^{\prime}$ is a motivic Hecke character of $\mathscr{K}^{\prime}$. Indeed, $\omega$ is motivic, $\omega \mid H_{E}(\mathbb{R}) \equiv 1$ (notation 1.1); thus we can write $\omega=\eta \cdot \omega_{0}$, where $\eta$ is a character of finite order and $\omega_{0}$ is trivial on $H_{E}(\mathbf{A})$. By Proposition $1.4(\mathrm{c})$, such a factorization, which is not unique, permits us to write

$$
p_{\mathscr{K}}(\omega, \Psi) \sim_{E_{\Psi} \cdot E\left(\omega_{0}\right) E(\eta) ; E_{\Psi}} p_{\mathscr{K}}(\eta, \Psi) \cdot p_{\mathscr{H}}\left(\omega_{0}, \Psi\right) .
$$

A simple descent argument thus reduces (A.1) and (A.2) to the corresponding assertions for $\eta$ and $\omega_{0}$.

Now $\omega_{0}$ factors through the map $\psi: H_{\mathscr{K}} \rightarrow T_{\mathscr{K}}$ of $\S 1.1$; by Proposition 1.4(a) we may replace $H_{\mathscr{K}}$ by $T_{\mathscr{K}}$. The canonical local systems of [8], which are used to define the rational structures on automorphic vector bundles on $\operatorname{Sh}\left(T_{\mathscr{K}}, \psi \circ h_{\Psi}\right)$ are obtained in two steps. First, we find a collection of pairs $\left\{\left(H_{\mathscr{H}^{\prime}}, h_{\Phi^{\prime}}\right)\right\}$, where $\mathscr{K}^{\prime}$ is a CM field and $\Phi^{\prime}$ is a CM type, which admits a map

$$
\Pi \operatorname{Sh}\left(H_{\mathscr{K}^{\prime}}, h_{\Phi^{\prime}}\right) \rightarrow \operatorname{Sh}\left(T_{\mathscr{K}}, \psi \circ h_{\Psi}\right),
$$

of Shimura varieties. Next, we descend the canonical local systems already constructed on $\Pi \operatorname{Sh}\left(H_{\mathscr{K}^{\prime}}, h_{\Phi^{\prime}}\right)$ to $\operatorname{Sh}\left(T_{\mathscr{K}}, \psi \circ h_{\Psi}\right)$ and prove that the result is independent of the choices made in (A.3). Applying Proposition 1.4(a) again, we may replace $\left(T_{\mathscr{K}}, \psi \circ h_{\Psi}\right)$ by pairs of the form $\left(H_{\mathscr{H}^{\prime}}, h_{\Phi^{\prime}}\right)$; in other words, we are reduced to checking (A.1) and (A.2) when $\Psi$ is a CM type.

But now if $\Psi$ is a CM type, then $\operatorname{Sh}\left(H_{\mathscr{K}}, h_{\Psi}\right)$ embeds in the HilbertBlumenthal variety $\mathscr{M}_{E}$, which is the Shimura variety attached to $R_{E / \mathbb{Q}} G L(2)_{E}$ and the $d$-fold product of $(\mathbb{C}-\mathbb{R})$. Let $i: H_{\mathscr{K}} \rightarrow R_{E / \mathbb{Q}} G L(2)_{E}$ be the corresponding embedding; up to conjugation, we may assume $i\left(H_{\mathscr{K}}\right)$ is normalized by the element

$$
\delta_{0}=\left(\begin{array}{cc}
-1 & 0 \\
0 & 1
\end{array}\right) \in G L(2, E) .
$$

Let $\mathscr{J}=\mathscr{J}\left(\delta_{0}\right)$ be the corresponding operator on automorphic forms on $G L(2, E)$. Note that $l \circ \mathcal{J}$ extends to an involution of the field of meromorphic automorphic forms. Now for any Hecke character $\omega$ of $\mathscr{K}$, there obviously exists a meromorphic automorphic form $F$ on $G L(2, E)$, rational over $\mathbb{Q}$, such that $L_{\omega}(F)$ is well defined and not equal to zero. Tracing back the proof of Lemma 2.4.5, we see that (A.1) and (A.2) for $\operatorname{Sh}\left(H_{\mathscr{K}}, h_{\Psi}\right)$ are equivalent to Lemma 2.4 .5 for the embedding of $\operatorname{Sh}\left(H_{\mathscr{K}}, h_{\Psi}\right)$ in $\mathscr{M}_{E}$, with $\mathscr{J}=\mathscr{J}\left(\delta_{0}\right)$. But this case has been proved by Shimura (cf. [9, I, Lemma 1.4.6]).

This completes the argument for $\omega_{0}$. Formulas (A.1) and (A.2) for the finite character $\eta$ follow easily from the reciprocity law for the profinite Shimura 
variety $\operatorname{Sh}\left(H_{\mathscr{K}}, h_{\Psi}\right)$ itself (i.e., no reference to abelian varieties is necessary); we omit the elementary proof. A less elementary argument, but one that is perhaps more enlightening, goes as follows. Just as above, we can trace back the proof of Lemma 2.4.5, but now applied to the structure sheaf $\mathscr{O}=\mathscr{E}_{(\underline{0}, 0)}^{D}$ of $\operatorname{Sh}\left(D^{\times},\left(\mathfrak{H}^{ \pm}\right)^{\Sigma(D)}\right), \underline{0}=(0, \ldots, 0)$; we find that formulas (A.1) and (A.2) are a consequence of Lemma 2.4.5 for the special case of modular forms of weight $(\underline{0}, 0)$. But this special case is just Langlands' conjecture on the action of complex conjugation on $\operatorname{Sh}\left(D^{\times},\left(\mathfrak{H}^{ \pm}\right)^{\Sigma(D)}\right)$, stated and proved as Conjecture $B$ in [4] by Milne and Shih.

\section{ACKNOWLEDGMENTS}

I learned most of what I know about the theta correspondence from S. Kudla, and many of the techniques used in the present paper were developed during the course of our longstanding collaboration. Most of what I did not learn from Kudla was explained to me by S. Rallis. I thank both of them for their support over many years, and for allowing me to cite their unpublished work on the Weil-Siegel formula for unitary groups.

The basic argument of this paper was developed while I was a visitor at the Steklov Institute of Mathematics in Moscow, in 1989-90. I thank the Algebra Department of the Steklov Institute for its hospitality, and acknowledge the support of the National Academy of Sciences in providing the opportunity to visit Moscow as an exchange scholar. During the preparation of this paper, J. Rogawski explained to me the subtleties of splittings of metaplectic covers, and J.-S. Li answered a number of questions regarding his work on Rallis' inner product formula and standard $L$-functions. G. Shimura kindly clarified the history of his conjectures and their relation to other questions. I also had stimulating discussions on the subject of this paper with D. Blasius, B. Gross, T. Oda, A. A. Panchishkin, and D. Ramakrishnan. I thank the referee for pointing out a number of loose ends at different points in the argument. Finally, I would like to thank P. Garrett for proposing a bet that led me to the basic idea of this paper. Suffice it to say that nobody won the bet.

\section{REFERENCES}

1. D. Blasius, On the critical values of Hecke L-series, Ann. of Math. (2) 124 (1986), 23-63.

2. __ Period relations and values of automorphic L-functions, manuscript, 1987.

3. M. Borovoí, Langlands' conjecture concerning conjugation of connected Shimura varieties, Selecta Math. Soviet 3 (1984), 3-39.

4. P. Deligne, J. S. Milne, A. Ogus, and K.-Y. Shih, Hodge cycles, motives, and Shimura varieties, Lecture Notes in Math., vol. 900, Springer-Verlag, Berlin, Heidelberg, and New York, 1982.

5. P. B. Garrett and M. Harris, Special values of triple product L-functions, Amer. J. Math. (to appear).

6. S. Gelbart, I. Piatetski-Shapiro, and S. Rallis, Explicit constructions of automorphic Lfunctions, Lecture Notes in Math., vol. 1254, Springer-Verlag, Berlin, Heidelberg, and New York, 1987.

7. S. Gelbart and J. Rogawski, L-functions and Fourier-Jacobi coefficients for the unitary group $U(3)$, Invent. Math. 105 (1991), 445-472. 
8. M. Harris, Arithmetic vector bundles and automorphic forms on Shimura varieties. I, Invent. Math. 82 (1985), 151-189; II, Compositio Math. 60 (1986), 323-378.

9. __ Period invariants of Hilbert modular forms. I, Lecture Notes in Math., vol. 1447, Springer-Verlag, Berlin, Heidelberg, and New York, 1990, 155-202; II, manuscript, 1992.

10. _ Non-vanishing of L-functions of $2 \times 2$ unitary groups, Forum Math. (to appear).

11. _ L-functions and periods of polarized regular motives, manuscript, 1991.

12. M. Harris and S. Kudla, Arithmetic automorphic forms for the nonholomorphic discrete series of $G \mathrm{Sp}(2)$, Duke Math. J. 66 (1992), 59-122.

13. 605-672.

14. R. Howe, $\theta$-series and invariant theory, Proc. Sympos. Pure Math., vol. 33, Amer. Math. Soc., Providence, RI, 1979, pp. 275-286.

15. _ Transcending classical invariant theory, J. Amer. Math. Soc. 2 (1989), 535-552.

16. H. Jacquet, Automorphic forms on GL(2) . II, Lecture Notes in Math., vol. 278, SpringerVerlag, Berlin, Heidelberg, and New York, 1972.

17. H. Jacquet and R. P. Langlands, Automorphic forms on $G L(2)$, Lecture Notes in Math., vol. 114, Springer-Verlag, Berlin, Heidelberg, and New York, 1970.

18. U. Jannsen, Mixed motives and algebraic K-theory, Lecture Notes in Math., vol. 1400, Springer-Verlag, Berlin, Heidelberg, and New York, 1990.

19. M. Kashiwara and M. Vergne, On the Segal-Shale-Weil representations and harmonic polynomials, Invent. Math. 44 (1978), 1-47.

20. S. S. Kudla, On certain arithmetic automorphic forms for $S U(1, q)$, Invent. Math. 52 (1979), 1-25.

21. ___ Seesaw dual reductive pairs, Automorphic forms of Several Variables, Taniquchi symposium, Katata 1983, Birkhaüser-Verlag, Boston, 1984, pp. 244-268.

22. S. S. Kudla and S. Rallis, On the Weil-Siegel formula, J. Reine Angew. Math. 387 (1988), 1-68; II. The isotropic convergent case, J. Reine Angew. Math. 391 (1988), 65-84.

23. __ Poles of Eisenstein series and L-functions, Festschrift in Honor of I. I. PiatetskiShapiro, Israel Math. Conf. Proc. 3 (1990), 81-110.

24. __ A regularized Siegel-Weil formula: the first term identity, preprint (1992).

25. J.-P. Labesse and R. P. Langlands, L-indistinguishability for $S L(2)$, Canad. J. Math. 31 (1979), 726-785.

26. J.-S. Li, Non-vanishing theorems for the cohomology of certain arithmetic quotients, J. Reine Angew. Math. 428 (1992), 177-217.

27. J. S. Milne, Canonical models of (mixed) Shimura varieties and automorphic vector bundles, Proc. Ann Arbor Conf. on Shimura Varieties and Automorphic Forms, Progr. Math., vol. 1, Academic Press, New York, 1989, pp. 283-414.

28. C. Moeglin, M.-F. Vigneras, and J.-L. Waldspurger, Correspondence de Howe sur un corps p-adique, Lecture Notes in Math., vol. 1291, Springer-Verlag, Berlin, Heidelberg, and New York, 1987.

29. T. Oda, Periods of Hilbert modular surfaces, Progr. Math., vol. 19, Birkhaüser, Boston, 1982.

30. _ Hodge structures of Shimura varieties attached to the unit groups of quaternion algebras, Galois Groups and Their Representations, Adv. Stud. Pure Math., vol. 2, NorthHolland, Amsterdam, 1983, pp. 15-36.

31. A. A. Panchishkin, Motives over totally real fields and p-adic L-functions, manuscript, 1990.

32. I. I. Piatetski-Shapiro and S. Rallis, $\varepsilon$-factor of representations of classical groups, Proc. Nat. Acad. Sci. U.S.A. 83 (1986), 4589-4593.

33. D. Prasad, Trilinear forms for representations of $G L(2)$ and local $\varepsilon$-factors, Compositio Math. 75 (1990), 1-46. 
34. S. Rallis, L-functions and the oscillator representation, Lecture Notes in Math., vol. 1245, Springer-Verlag, Berlin, Heidelberg, and New York, 1987.

35. H. Shimizu, Theta series and automorphic forms on $G L_{2}$, J. Math. Soc. Japan 24 (1972), 638-683.

36. G. Shimura, On canonical models of arithmetic quotients of bounded symmetric domains. I, Ann. of Math. (2) 91 (1970), 144-222; II, 92 (1970), 528-549.

37. $\ldots$, The special values of the zeta functions associated with Hilbert modular forms, Duke Math. J. 45 (1978), 637-679.

38. __, Automorphic forms and the periods of abelian varieties, J. Math. Soc. Japan 31 (1979), 561-592.

39. - The arithmetic of certain zeta functions and automorphic forms on orthogonal groups, Ann. of Math. (2) 111 (1980), 313-375.

40. _ On certain zeta functions attached to two Hilbert modular forms. I, II, Ann. of Math. (2) 114 (1981), 127-164, 569-607.

41. _ The critical values of certain zeta functions associated with modular forms of halfintegral weight, J. Math. Soc. Japan 33 (1981), 649-672.

42. __ Algebraic relations between critical values of zeta functions and inner products, Amer. J. Math. 105 (1983), 253-285.

43. _ On the critical values of certain Dirichlet series and the periods of automorphic forms, Invent. Math. 94 (1988), 245-305.

44. J. Tunnell, Local $\varepsilon$-factors and characters of $G L_{2}$, Amer. J. Math. 105 (1983), 1277-1308.

45. J.-L. Waldspurger, Démonstration d'une conjecture de dualité de Howe dans le cas p-adique, $p \neq 2$, Festschrift in Honor of I. I. Piatetski-Shapiro, Israel Math. Conf. Proc. 2 (1990), 267-324.

46. __ Sur les valeurs de certains fonctions $L$ automorphes en leur centre de symétrie, Compositio Math. 54 (1985), 173-242.

47. __ Quelques propriétés arithmétiques de certaines formes automorphes sur GL(2), Compositio Math. 54 (1985), 121-171.

48. __ Correspondance de Shimura et quaternions, Forum Math. 3 (1991), 219-307.

49. A. Weil, Sur la formule de Siegel dans la théorie des groupes classiques, Acta Math. 113 (1965), 1-87.

50. H. Saito, On Tunnell's formula for characters of GL(2), manuscript, 1991.

51. S. Kudla, Splitting metaplectic covers of dual reductive pairs, manuscript, 1991.

52. S. Rallis, On the Howe duality conjecture, Compositio Math. 51 (1984), 333-399.

Department of Mathematics, Brandeis University, Waltham, Massachusetts 02254 